In cooperation with Oakland County, Michigan

\title{
Water Resources Data, Oakland County, Michigan 2001-2004
}

Open File Report 2004-1417 


\section{Water Resources Data, Oakland County, Michigan 2001-2004}

By S.S. Aichele, S.L.Crowley, C.K.Taricska, J. Stopar

In cooperation with Oakland County, Michigan

Open File Report 2004-1417 


\section{U.S. Department of the Interior \\ Gale A. Norton, Secretary \\ U.S. Geological Survey \\ Charles G. Groat, Director}

U.S. Geological Survey, Reston, Virginia: 2005

For sale by U.S. Geological Survey, Information Services
Box 25286, Denver Federal Center
Denver, CO 80225
For more information about the USGS and its products:
Telephone: 1-888-ASK-USGS
World Wide Web: http://www.usgs.gov/

\footnotetext{
Any use of trade, product, or firm names in this publication is for descriptive purposes only and does not imply endorsement by the U.S. Government.

Although this report is in the public domain, permission must be secured from the individual copyright owners to reproduce any copyrighted materials contained within this report.

Suggested citation:

Aichele, S.S., S.L. Crowley, C.K. Taricska, and J. Stopar, 2005 Water Resources Data, Oakland County, Michigan 20012004 : U.S. Geological Survey Open File Report 2004-1417, 10p.
} 


\section{Contents}

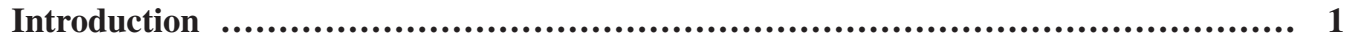

Methods ............................................................................... 1

Monitoring ................................................................... 1

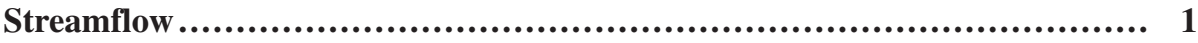

Ground-Water Levels $\quad$......................................................... 3

Continuous Stream Water Quality ........................................... 4

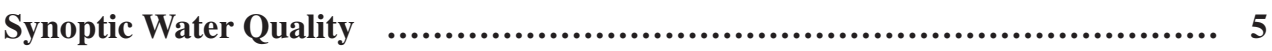

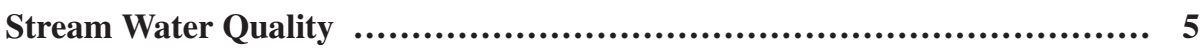

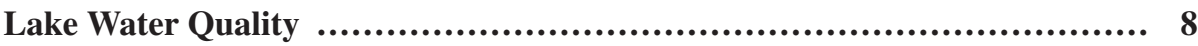

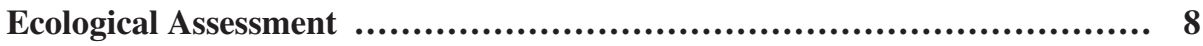

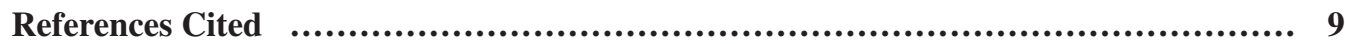

\section{Figures}

1-5 Maps showing:

1. Locations of continuous streamflow gages in and around Oakland

County, Michigan .............................................................. 2

2. Locations of ground-water monitoring wells in Oakland County, Michigan ........................................................................ 3

3. Locations of continuous stream water-quality monitoring stations in Oakland County, Michigan ................................................... 4

4. Locations of stream water-quality sampling sites in and around Oakland County, Michigan .................................................. 5

5 Locations of lake water-quality sampling sites in Oakland County, Michigan ................................................................... 8

\section{Tables}

1. Station number, name, and location of streamflow gages in and around Oakland County, Michigan.

2. Station number, name, and location of ground-water monitoring wells in Oakland County, Michigan.

3. Station number, name, and location of stream-water-quality monitoring stations in Oakland County, Michigan.

4. Station number, name, and location of stream-water-quality sampling sites in and around Oakland County, Michigan.

5. Water-quality characteristics and analytical methods for inorganic stream-water-quality samples collected in Oakland County, Michigan during 2001, 2002, and 2003.

6. Station number, name, and location of lake-water-quality sampling sites in Oakland County, Michigan 
7. Daily mean and maximum depth, in feet, of water below the land surface in four monitoring wells in Oakland County, Michigan, June 2001-March

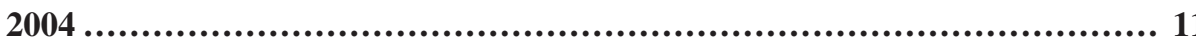

8. Measured depth, in feet, to water in monitoring well 424133083293201 , Oakland County, Michigan .................................................... 34

9. Results of synoptic stream-water-quality sample analysis in Oakland County, Michigan between September 2001 and September 2003 35

10. Results of analysis for selected organic compounds in surface water at selected sites in Oakland County, Michigan in August, 2002 and September, 2003

11. Vertical profiles of field-measured characteristics of selected lakewater-quality sites in Oakland County, Michigan measured during 2002 and 2003. 66

12. Results of chemical-water-quality analyses for selected parameters at selected lakes in Oakland County, Michigan, 2002 - 2003

13. Results of ecological assessment at selected stream channel locations in Oakland County, Michigan, September 2003

\section{Conversion Factors and Datum}

\begin{tabular}{|c|c|c|}
\hline Multiply & By & To obtain \\
\hline \multicolumn{3}{|c|}{ Length } \\
\hline inch (in.) & 2.54 & centimeter $(\mathbf{c m})$ \\
\hline foot $(\mathrm{ft}$ & 0.3048 & meter $(\mathbf{m})$ \\
\hline mile (mi) & 1.609 & kilometer (km) \\
\hline yard $(y d)$ & 0.9144 & meter $(\mathbf{m})$ \\
\hline \multicolumn{3}{|c|}{ Area } \\
\hline acre & 4,047 & square meter (m2) \\
\hline section ( 640 acres or 1 square mile) & 259.0 & square hectometer (hm2) \\
\hline square mile (mi2) & 259.0 & hectare (ha) \\
\hline square mile (mi2) & 2.590 & square kilometer (km2) \\
\hline \multicolumn{3}{|c|}{ Volume } \\
\hline cubic foot per second ( $\mathrm{ft} \mathbf{3} / \mathrm{s})$ & 0.02832 & cubic meter per second (m3/s) \\
\hline million gallons per day (Mgal/d) & 0.04381 & cubic meter per second $(\mathrm{m} 3 / \mathrm{s})$ \\
\hline
\end{tabular}

Temperature in degrees Celsius $\left({ }^{\circ} \mathrm{C}\right)$ may be converted to degrees Fahrenheit $\left({ }^{\circ} \mathrm{F}\right)$ as follows: ${ }^{\circ} \mathrm{F}=\left(\mathbf{1 . 8} \times{ }^{\circ} \mathrm{C}\right)+32$

Vertical coordinate information is referenced to the North American Vertical Datum of 1988 (NAVD 88).

Horizontal coordinate information is referenced to the North American Datum of 1983 (NAD 83). Altitude, as used in this report, refers to distance above the vertical datum.

Specific conductance is given in microsiemens per centimeter at 25 degrees Celsius $\left(\mu \mathrm{S} / \mathrm{cm}\right.$ at $\left.25{ }^{\circ} \mathrm{C}\right)$. Concentrations of chemical constituents in water are given either in milligrams per liter $(\mathrm{mg} / \mathrm{L})$ or micrograms per liter $(\mu \mathrm{g} / \mathrm{L})$. 


\title{
Water Resources Data for Oakland County, Michigan 2001-2004
}

\author{
By S.S. Aichele, S.L. Crowley, C.K. Taricska, and J. Stopar
}

\section{Introduction}

The U.S. Geological Survey (USGS), in cooperation with Oakland County, the Huron-Clinton Metropark Authority, the Michigan Department of Environmental Quality (MDEQ), and the Rouge Program Office, collected streamflow, water-quality, and ground-water-level data in watersheds across Oakland County during water years 2001, 2002, 2003, and 2004. Water years begin October 1 and end September 30.

This report presents water resources data used to produce a series of interpretive reports on the quantity and quality of water in Oakland County for Oakland County, Michigan. Some of these data have been published elsewhere, but are provided here in one report. This report has two main sections. The first section provides an overview of the methods used to collect the various types of data. The second section is a series of data tables containing ground-water-level data, synoptically measured stream-water-quality data, synoptically measured lake-water-quality data, and the results of a macroinvertebrate and habitat assessment.

\section{Methods}

Data collection activities in Oakland County during water years 2001-2004 can be divided into two general classes: routine monitoring and synoptic water-quality measurement. Routine monitoring activities were carried out in conjunction with ongoing Federal and State monitoring programs, using methods appropriate to those programs. Surface-water monitoring data are available in the USGS Michigan District Annual Data
Report (Blumer and others, 2002; 2003; 2004) from the appropriate year. Ground-water monitoring data are provided in this volume. More detailed discussion of monitoring protocols can also be found in the Annual Data Reports and in Wagner and others (2000).

\section{Monitoring}

Continuous monitoring activities in this project involved monitoring streamflow at 12 stations in and around Oakland County, monitoring temperature and specific conductance of stream water at 7 stations, and monitoring ground-water levels in 5 wells.

\section{Streamflow}

Continuous measurements of stream stage were made at streamflow-gaging stations in and around the county. Figure 1 shows the locations of these stations, and table 1 presents the station location information. Streamflow was calculated using site-specific stage-discharge ratings in accordance with standard USGS procedures described in Rantz and others (1982). The majority of these stations are maintained as part of ongoing Federal, State, and county monitoring activities. USGS streamflow stations 04143900 (Shiawassee River near Linden, Mich.) and 04161000 (Clinton River at Auburn Hills, Mich.) were operated from June 2001 through September 2003 specifically for this project. During the period from October 1, 2002 to September 30, 2003 only stage data was collected for station 04161000. Streamflow data are available in the USGS Michigan District Annual Data Report and online at http://mi.water.usgs. gov. 


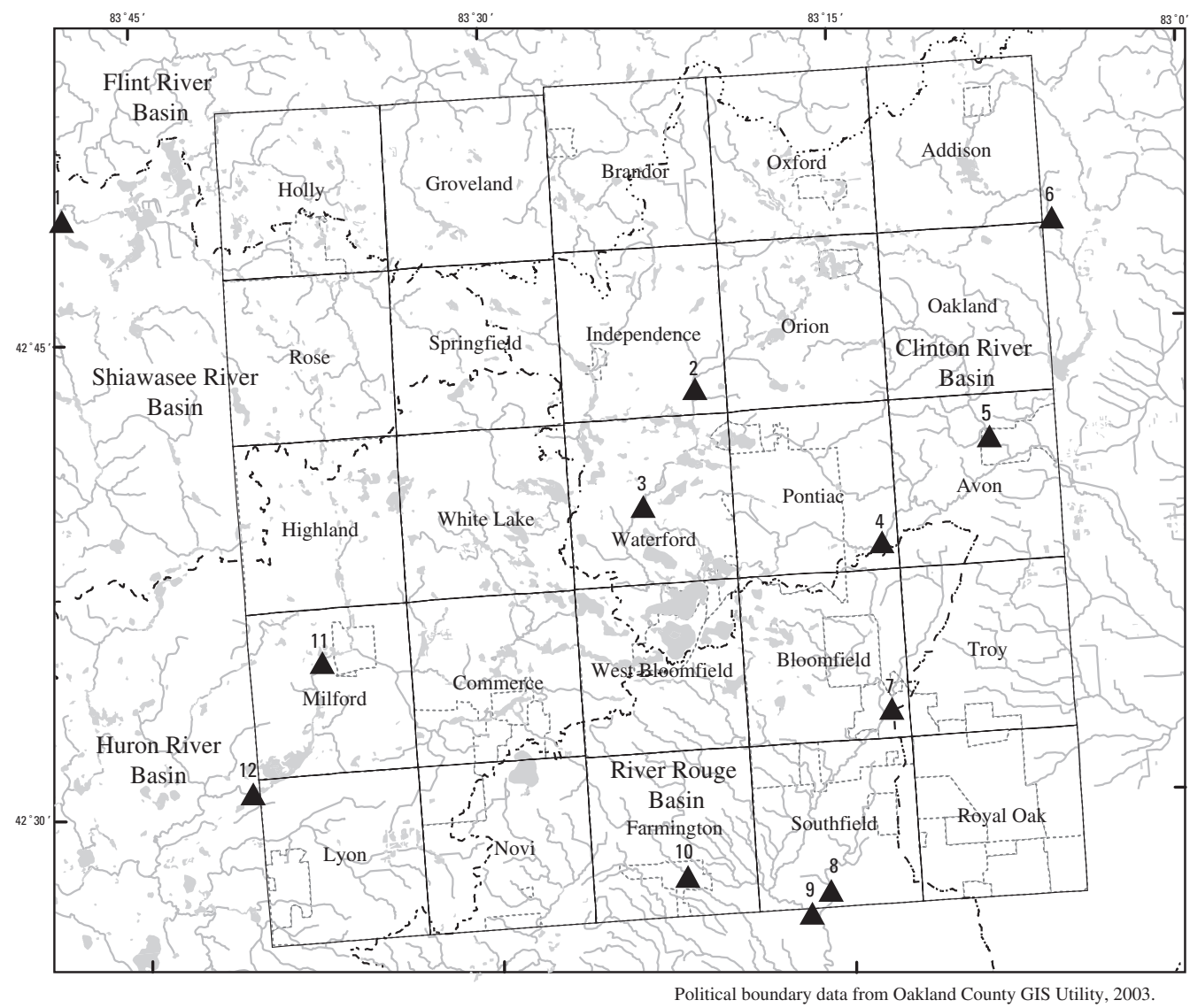

EXPLANATION

1 Streamflow gaging station location and reference number

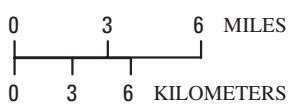
Hydrographic data from USGS 1:100,000 scale Digital Line Graphs.

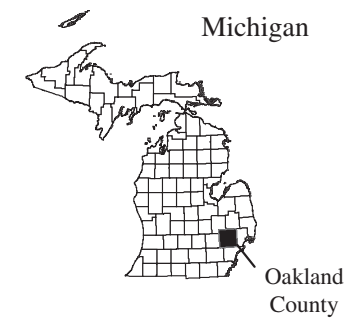

Figure 1. Locations of continuous streamflow gages in and around Oakland County, Michigan.

(Station information can be obtained by crossreferencing the number in this figure to table 1 of this report.)

Table 1. Station number, name, and location of streamflow gages in and around Oakland County, Michigan.

[Reference number corresponds to number on figure 1 of this report]

\begin{tabular}{|c|c|c|c|c|c|}
\hline $\begin{array}{l}\text { Reference } \\
\text { number }\end{array}$ & $\begin{array}{l}\text { Station } \\
\text { number }\end{array}$ & Station name & Latitude & Longitude & $\begin{array}{l}\text { Drainage area } \\
\text { (square miles) }\end{array}$ \\
\hline 1 & 04143900 & Shiawassee River at Linden, Mich. & $42^{\circ} 48^{\prime} 57^{\prime \prime} \mathrm{N}$ & $83^{\circ} 48^{\prime} 07^{\prime \prime} \mathrm{W}$ & 83.7 \\
\hline 2 & 04160800 & Sashabaw Creek near Drayton Plains, Mich. & $42^{\circ} 43^{\prime} 12^{\prime \prime} \mathrm{N}$ & $83^{\circ} 21^{\prime} 13^{\prime \prime} \mathrm{W}$ & 20.9 \\
\hline 3 & 04160900 & Clinton River Near Drayton Plains, Mich. & $42^{\circ} 39^{\prime} 37^{\prime \prime} \mathrm{N}$ & $83^{\circ} 23^{\prime} 25^{\prime \prime} \mathrm{W}$ & 79.2 \\
\hline 4 & 04161000 & Clinton River at Auburn Hills, Mich. & $42^{\circ} 38^{\prime} 00^{\prime \prime} \mathrm{N}$ & $83^{\circ} 13^{\prime} 28^{\prime \prime} \mathrm{W}$ & 123.0 \\
\hline 5 & 04161540 & Paint Creek at Rochester, Mich. & $42^{\circ} 41^{\prime} 18^{\prime \prime} \mathrm{N}$ & $83^{\circ} 08^{\prime} 35^{\prime \prime} \mathrm{W}$ & 70.9 \\
\hline 6 & 04166000 & River Rouge at Birmingham, Mich. & $42^{\circ} 32^{\prime} 45^{\prime \prime} \mathrm{N}$ & $83^{\circ} 13^{\prime} 25^{\prime \prime} \mathrm{W}$ & 33.3 \\
\hline 7 & 04166100 & River Rouge at Southfield, Mich. & $42^{\circ} 26^{\prime} 52^{\prime \prime} \mathrm{N}$ & $83^{\circ} 17^{\prime} 52^{\prime} \mathrm{W}$ & 87.9 \\
\hline 8 & 04161580 & Stony Creek near Romeo, Mich. & $42^{\circ} 48^{\prime} 03^{\prime \prime} \mathrm{N}$ & $83^{\circ} 05^{\prime} 25^{\prime \prime} \mathrm{W}$ & 25.6 \\
\hline 9 & 04166200 & Evans Ditch at Southfield, Mich. & $42^{\circ} 27^{\prime} 28^{\prime \prime} \mathrm{N}$ & $83^{\circ} 16^{\prime} 03^{\prime \prime} \mathrm{W}$ & 9.5 \\
\hline 10 & 04166300 & Upper River Rouge at Farmington, Mich. & $42^{\circ} 27^{\prime} 52^{\prime \prime} \mathrm{N}$ & $83^{\circ} 22^{\prime} 11^{\prime \prime} \mathrm{W}$ & 17.5 \\
\hline 11 & 04170000 & Huron River at Milford, Mich. & $42^{\circ} 34^{\prime} 44^{\prime \prime} \mathrm{N}$ & $83^{\circ} 37^{\prime} 36^{\prime \prime} \mathrm{W}$ & 132.0 \\
\hline 12 & 04170500 & Huron River near New Hudson, Mich. & $42^{\circ} 30^{\prime} 45^{\prime \prime} \mathrm{N}$ & $83^{\circ} 40^{\prime} 35^{\prime \prime} \mathrm{W}$ & 148.0 \\
\hline
\end{tabular}




\section{Ground-Water Levels}

Ground-water levels were monitored in five wells in the western part of Oakland County. Figure 2 shows locations of these monitoring wells and table 2 presents the well location information. Four wells were instrumented with pressure transducers and electronic data loggers, recording water levels every hour. The fifth well was monitored periodically, using an electric tape. Water-level measurements are reported in depth below land surface datum (LSD). Water levels are reported as both daily mean and maximum in table 7 . Measurements made at well 424133083293201 are presented in table 8. These data also are available in the USGS Michigan District Annual Data Report and online at http://mi.water.usgs.gov.

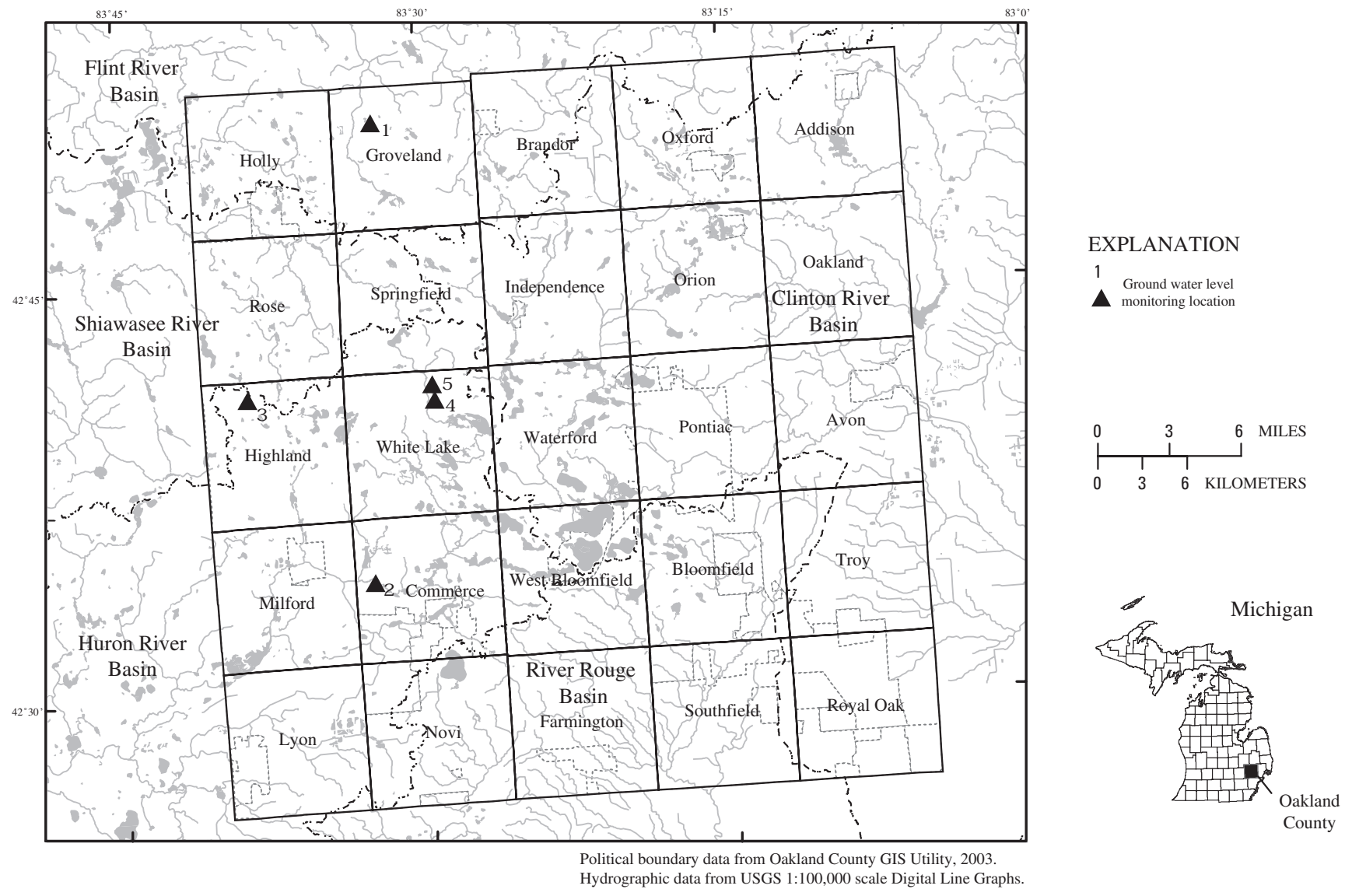

Figure 2. Locations of ground-water monitoring wells in Oakland County, Michigan.

(Station names can be obtained by cross referencing the number in this figure to table 2 of this report.)

Table 2. Station number, name, and location of ground-water monitoring wells in Oakland County, Michigan.

[Reference number corresponds to number on figure 2 of this report]

\begin{tabular}{|c|c|c|c|c|c|}
\hline $\begin{array}{l}\text { Reference } \\
\text { number }\end{array}$ & Station number & Station name & Longitude & Latitude & $\begin{array}{l}\text { Well depth, } \\
\text { in feet }\end{array}$ \\
\hline 1 & 425116083321501 & Holly State Recreation Area & $42^{\circ} 51^{\prime} 09^{\prime \prime} \mathrm{N}$ & $83^{\circ} 32^{\prime} 15^{\prime \prime} \mathrm{W}$ & 42 \\
\hline 2 & 423423083324001 & Proud Lake State Recreation Area & $42^{\circ} 34^{\prime} 23^{\prime \prime} \mathrm{N}$ & $83^{\circ} 32^{\prime} 40^{\prime \prime} \mathrm{W}$ & 45 \\
\hline 3 & 424109083384301 & Fish Lake Road & $42^{\circ} 41^{\prime} 09^{\prime \prime} \mathrm{N}$ & $83^{\circ} 38^{\prime} 44^{\prime \prime} \mathrm{W}$ & 49 \\
\hline 4 & 424133083293101 & Teggerdine Road & $42^{\circ} 41^{\prime} 00^{\prime \prime} \mathrm{N}$ & $83^{\circ} 29^{\prime} 29^{\prime \prime} \mathrm{W}$ & 175 \\
\hline 5 & 424133083293201 & White Lake Road & $42^{\circ} 41^{\prime} 34{ }^{\prime \prime} \mathrm{N}$ & $83^{\circ} 29^{\prime} 33^{\prime \prime} \mathrm{W}$ & 163 \\
\hline
\end{tabular}




\section{Continuous Stream Water Quality}

Stream temperature and specific conductance were monitored continuously at seven stations around Oakland County, in collaboration with other monitoring programs. Monitoring was performed in accordance with standard USGS procedures as described in Wagner and others (2000). Figure 3 shows the locations of these continuous stream water-quality monitoring stations in Oakland County, and table 3 presents the station location information. These data are available in the USGS Michigan District Annual Data Report and online at http://mi.water.usgs.gov.

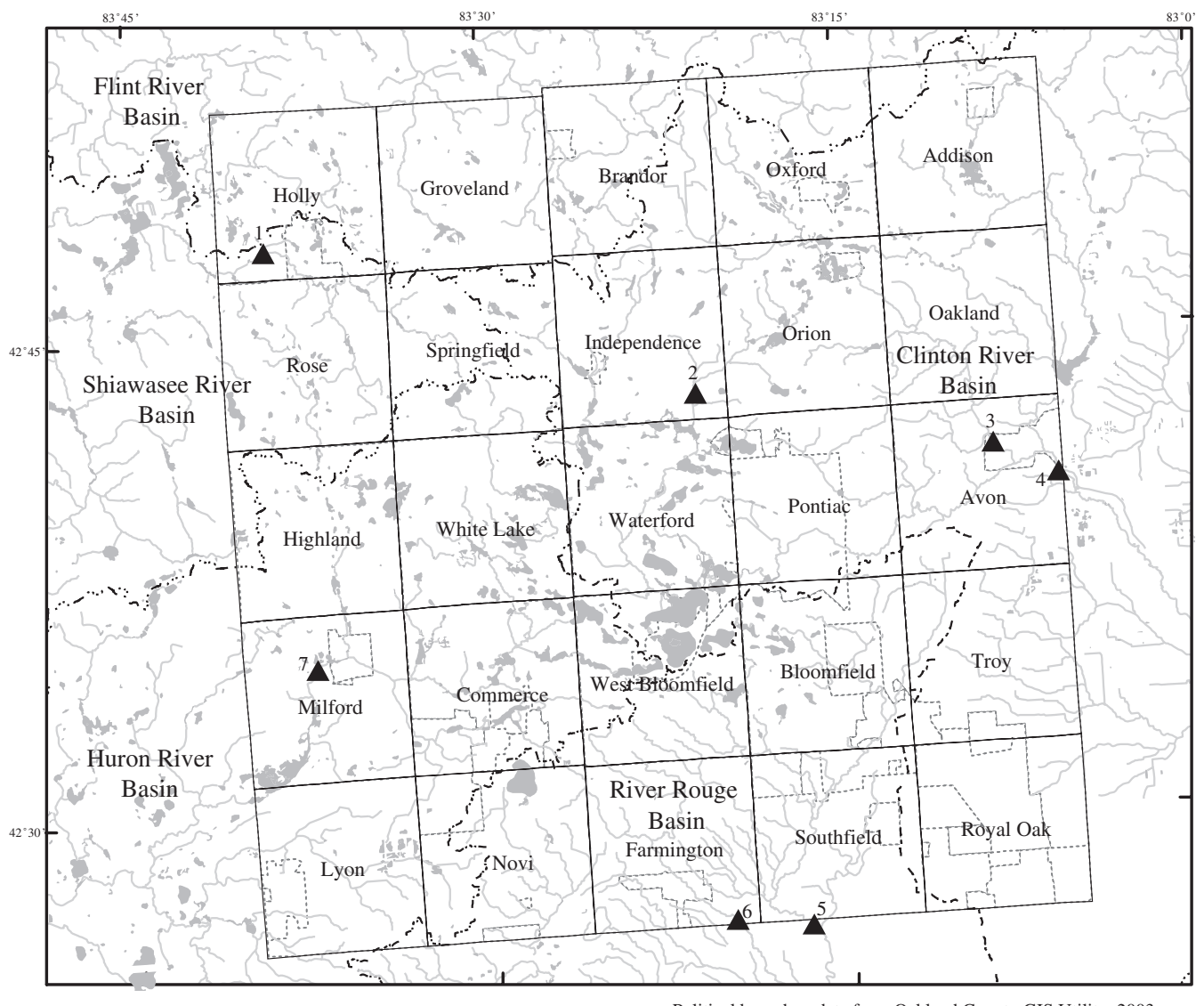

EXPLANATION

1 Continuous stream water quality

A monitoring locations
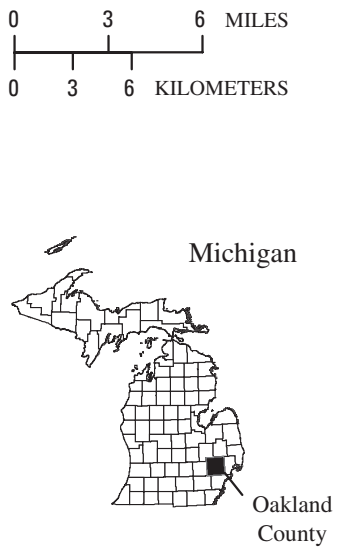

Figure 3. Locations of continuous stream-water-quality monitoring stations in Oakland County, Michigan. (Station information can be obtained by cross referencing the number in this figure to table 3 of this report.)

Table 3. Station number, name, and location of stream water-quality monitoring stations in Oakland County, Michigan.

[Reference number corresponds to number on figure 3 of this report]

\begin{tabular}{|c|c|c|c|c|c|}
\hline $\begin{array}{l}\text { Reference } \\
\text { number }\end{array}$ & $\begin{array}{l}\text { Station } \\
\text { number }\end{array}$ & Station name & Latitude & Longitude & $\begin{array}{l}\text { Drainage area } \\
\text { (square miles) }\end{array}$ \\
\hline 1 & 04143830 & Shiawassee River at Holly, Mich. & $42^{\circ} 47^{\prime} 57^{\prime \prime} \mathrm{N}$ & $83^{\circ} 38^{\prime} 52^{\prime \prime} \mathrm{W}$ & 49.2 \\
\hline 2 & 04160800 & Sashabaw Creek near Drayton Plains, Mich. & $42^{\circ} 43^{\prime} 12^{\prime \prime} \mathrm{N}$ & $83^{\circ} 21^{\prime} 13^{\prime \prime} \mathrm{W}$ & 20.9 \\
\hline 3 & 04161540 & Paint Creek at Rochester, Mich. & $42^{\circ} 41^{\prime} 18^{\prime \prime} \mathrm{N}$ & $83^{\circ} 08^{\prime} 35^{\prime \prime} \mathrm{W}$ & 70.9 \\
\hline 4 & 04161810 & Clinton River at Yates, Mich. & $42^{\circ} 40^{\prime} 18^{\prime \prime} \mathrm{N}$ & $83^{\circ} 05^{\prime} 47^{\prime \prime} \mathrm{W}$ & 299.0 \\
\hline 5 & 04166100 & River Rouge at Southfield, Mich. & $42^{\circ} 26^{\prime} 52^{\prime \prime} \mathrm{N}$ & $83^{\circ} 17^{\prime} 52^{\prime \prime} \mathrm{W}$ & 87.9 \\
\hline 6 & 04166315 & Upper River Rouge at Clarenceville, Mich. & $42^{\circ} 26^{\prime} 48^{\prime \prime} \mathrm{N}$ & $83^{\circ} 20^{\prime} 12^{\prime \prime} \mathrm{W}$ & 19.8 \\
\hline 7 & 04170000 & Huron River at Milford, Mich. & $42^{\circ} 34^{\prime} 44^{\prime \prime} \mathrm{N}$ & $83^{\circ} 37^{\prime} 36^{\prime \prime} \mathrm{W}$ & 132.0 \\
\hline
\end{tabular}




\section{Synoptic Water Quality}

Synoptic water-quality samples were collected from 14 sites across Oakland County, 11 of which coincided with streamflow-gaging stations and 2 of which coincided with stream-water-quality monitoring stations in Oakland County from September 2001 through September 2003. During the summers of 2002 and 2003, water-quality samples were also collected from 12 lake basins.

\section{Stream Water Quality}

The USGS collected 193 synoptic water-quality samples at 14 stream sites across Oakland County during the period from September, 2001 through September, 2003. A map showing the location of these sites is shown in figure 4 , while table 4 presents a listing of these sites with station number and location information. Results of inorganic and microbial analysis are presented in table 9. Results of inorganic analysis are presented in table 10.

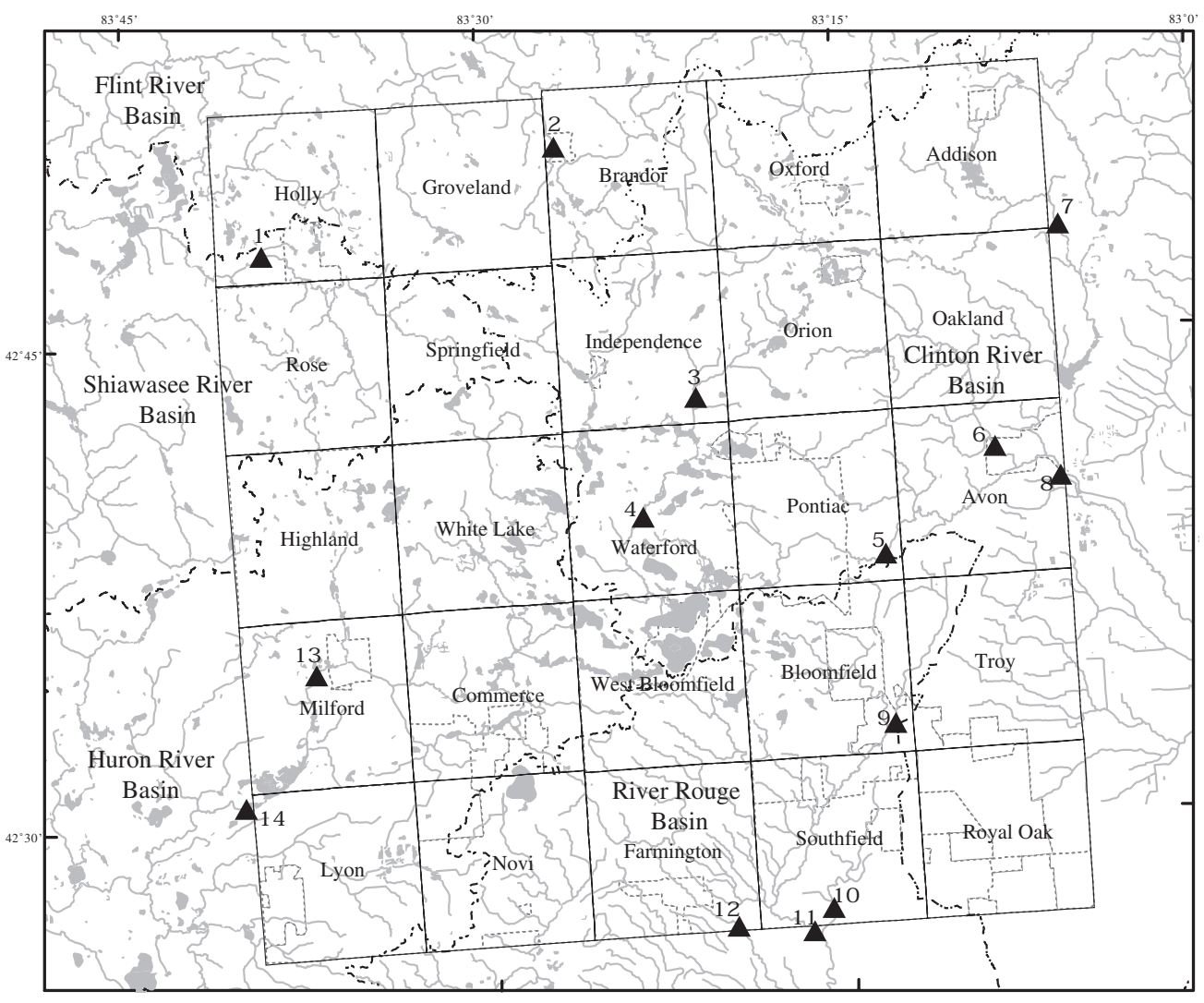

Political boundary data from Oakland County GIS Utility, 2003. Hydrographic data from USGS 1:100,000 scale Digital Line Graphs.

Figure 4. Locations of stream water-quality sampling sites in and around Oakland County, Michigan. (Site information can be obtained by cross referencing the number in the figure to table 4 of this report)

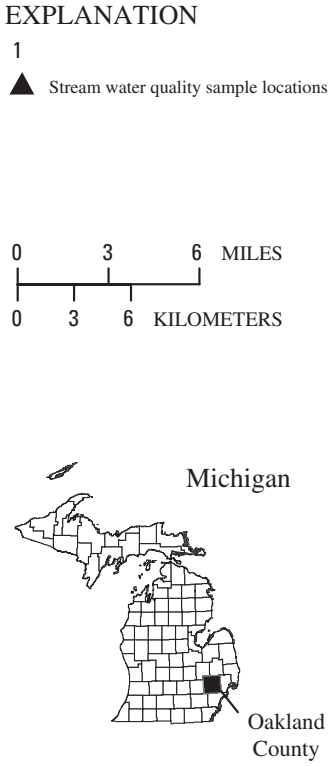

County 
Table 4. Station number, name, and location of water-quality sampling sites in and around Oakland County, Mich.

[Reference number corresponds to number in figure 4 of this report]

\begin{tabular}{|c|c|c|c|c|c|}
\hline $\begin{array}{l}\text { Reference } \\
\text { number }\end{array}$ & $\begin{array}{l}\text { Site } \\
\text { number }\end{array}$ & Site name & Latitude & Longitude & $\begin{array}{l}\text { Drainage area } \\
\text { (square miles) }\end{array}$ \\
\hline 1 & 04143830 & Shiawassee River at Holly, Mich. & $42 \circ 47^{\prime} 57^{\prime \prime} \mathrm{N}$ & $83^{\circ} 38^{\prime} 52^{\prime} \mathrm{W}$ & 49.2 \\
\hline 2 & 04148035 & Kearsley Creek at Mill Street at Ortonville, Mich. & $42^{\circ} 51^{\prime} 08^{\prime \prime} \mathrm{N}$ & $83^{\circ} 26^{\prime} 47^{\prime \prime} \mathrm{W}$ & 19.6 \\
\hline 3 & 04160800 & Sashabaw Creek near Drayton Plains, Mich. & $42^{\circ} 43^{\prime} 12^{\prime \prime} \mathrm{N}$ & $83^{\circ} 21^{\prime} 13^{\prime \prime} \mathrm{W}$ & 20.9 \\
\hline 4 & 04160900 & Clinton River near Drayton Plains, Mich. & $42^{\circ} 39^{\prime} 37^{\prime \prime} \mathrm{N}$ & $83^{\circ} 23^{\prime} 25^{\prime \prime} \mathrm{W}$ & 79.2 \\
\hline 5 & 04161000 & Clinton River at Auburn Hills, Mich. & $42^{\circ} 38^{\prime} 00^{\prime \prime} \mathrm{N}$ & $83^{\circ} 13^{\prime} 28^{\prime \prime} \mathrm{W}$ & 123.0 \\
\hline 6 & 04161540 & Paint Creek at Rochester, Mich. & $42^{\circ} 41^{\prime} 18^{\prime \prime} \mathrm{N}$ & $83^{\circ} 08^{\prime} 35^{\prime \prime} \mathrm{W}$ & 70.9 \\
\hline 7 & 04161810 & Clinton River at Yates, Mich. & $42^{\circ} 40^{\prime} 18^{\prime \prime} \mathrm{N}$ & $83^{\circ} 05^{\prime} 47^{\prime \prime} \mathrm{W}$ & 299.0 \\
\hline 8 & 04166000 & River Rouge at Birmingham, Mich. & $42^{\circ} 32^{\prime} 45^{\prime \prime} \mathrm{N}$ & $83^{\circ} 13^{\prime} 25^{\prime \prime} \mathrm{W}$ & 33.3 \\
\hline 9 & 04166100 & River Rouge at Southfield, Mich. & $42^{\circ} 26^{\prime} 52^{\prime \prime} \mathrm{N}$ & $83^{\circ} 17^{\prime} 52^{\prime \prime} \mathrm{W}$ & 87.9 \\
\hline 10 & 04161580 & Stony Creek near Romeo, Mich. & $42^{\circ} 48^{\prime} 03^{\prime \prime} \mathrm{N}$ & $83^{\circ} 05^{\prime} 25^{\prime \prime} \mathrm{W}$ & 25.6 \\
\hline 11 & 04166200 & Evans Ditch at Southfield, Mich. & $42^{\circ} 27^{\prime} 28^{\prime \prime} \mathrm{N}$ & $83^{\circ} 16^{\prime} 03^{\prime \prime} \mathrm{W}$ & 9.5 \\
\hline 12 & 04166315 & Upper River Rouge at Clarenceville, Mich. & $42^{\circ} 26^{\prime} 48^{\prime \prime} \mathrm{N}$ & $83^{\circ} 20^{\prime} 12^{\prime \prime} \mathrm{W}$ & 19.8 \\
\hline 13 & 04170000 & Huron River at Milford, Mich. & $42^{\circ} 34^{\prime} 44^{\prime \prime} \mathrm{N}$ & $83^{\circ} 37^{\prime} 36^{\prime \prime} \mathrm{W}$ & 132.0 \\
\hline 14 & 04170500 & Huron River near New Hudson, Mich. & $42^{\circ} 30^{\prime} 45^{\prime \prime} \mathrm{N}$ & $83^{\circ} 40^{\prime} 35^{\prime \prime} \mathrm{W}$ & 148.0 \\
\hline
\end{tabular}

Inorganic stream-water-quality samples were collected as depth and width integrated (DWI) samples using a DH-81 sampler as described in Wilde and others (1999). Streamflow was computed from stage-discharge ratings at all sites except 04143830, 04148035, and 04166315. At these three sites, streamflow measurements were made in accordance with standard
USGS procedures, as outlined in Rantz and others (1982). All samples were collected and handled in accordance with the procedures described in Wilde and others (2003) Wilde and others (1999), and Wilde and others (2004). A table of the parameters analyzed, the reporting level, and analytical methods is included in table 5. 
Table 5. Water-quality characteristics and analytical methods for inorganic stream water-quality samples collected in Oakland County, Michigan during 2001, 2002, and 2003

[MRL, Minimum Reporting Level; mg/L, milligrams per liter; $\mu \mathrm{g} / \mathrm{L}$, micrograms per liter; $\mu \mathrm{s} / \mathrm{cm}$, microsiemens per centimeter at 25 degrees Celsius]

\begin{tabular}{|c|c|c|c|c|c|}
\hline $\begin{array}{l}\text { Parameter } \\
\text { code }\end{array}$ & Characteristic & Units & MRL & Method & Reference \\
\hline 403 & $\mathrm{pH}$, laboratory & standard units & 0.1 & $\mathrm{I} 258785$ & Fishman and Friedman, 1989 \\
\hline 530 & Residue, total & $\mathrm{mg} / \mathrm{L}$ & 1.0 & I376585 & Fishman and Friedman, 1989 \\
\hline 608 & Nitrogen, ammonia & $\mathrm{mg} / \mathrm{L}$ as $\mathrm{N}$ & 0.02 & I252290 & Fishman, 1993 \\
\hline 613 & Nitrogen, nitrite & $\mathrm{mg} / \mathrm{L}$ as $\mathrm{N}$ & 0.01 & I254090 & Fishman, 1993 \\
\hline 623 & Nitrogen, ammonia \& organic & $\mathrm{mg} / \mathrm{L}$ as $\mathrm{N}$ & 0.1 & I261091 & Patton and Truitt, 1992 \\
\hline 625 & Nitrogen, ammonia \& organic & $\mathrm{mg} / \mathrm{L}$ as $\mathrm{N}$ & 0.1 & I451591 & Patton and Truitt, 2000 \\
\hline 631 & $\mathrm{NO} 2+\mathrm{NO} 3$, dissolved & $\mathrm{mg} / \mathrm{L}$ as $\mathrm{N}$ & 0.05 & I254590 & Fishman, 1993 \\
\hline 665 & Phosphorus, total & $\mathrm{mg} / \mathrm{L}$ as $\mathrm{P}$ & 0.05 & I461091 & Patton and Truitt, 1992 \\
\hline 666 & Phosphorus, dissolved & $\mathrm{mg} / \mathrm{L}$ as $\mathrm{P}$ & 0.004 & EPA365.1 & U.S. Environmental Protection Agency, 1994 \\
\hline 671 & Phosphorus, orthophosphate & $\mathrm{mg} / \mathrm{L}$ as $\mathrm{P}$ & 0.01 & I260190 & Fishman, 1993 \\
\hline 915 & Calcium, dissolved & $\mathrm{mg} / \mathrm{L}$ as $\mathrm{Ca}$ & 0.02 & I147287 & Fishman and Friedman, 1989 \\
\hline 925 & Magnesium, dissolved & $\mathrm{mg} / \mathrm{L}$ as $\mathrm{Mg}$ & 0.004 & $\mathrm{I} 147287$ & Fishman, 1993 \\
\hline 930 & Sodium, dissolved & $\mathrm{mg} / \mathrm{L}$ as $\mathrm{Na}$ & 0.06 & I147287 & Fishman, 1993 \\
\hline 935 & Potassium, dissolved & $\mathrm{mg} / \mathrm{L}$ as $\mathrm{K}$ & 0.1 & I163085 & Fishman and Friedman, 1989 \\
\hline 940 & Chloride, dissolved & $\mathrm{mg} / \mathrm{L}$ as $\mathrm{Cl}$ & 0.1 & I205785 & Fishman and Friedman, 1989 \\
\hline 945 & Sulfate, dissolved & $\mathrm{mg} / \mathrm{L}$ as $\mathrm{SO} 4$ & 0.1 & I205785 & Fishman and Friedman, 1989 \\
\hline 950 & Fluoride, dissolved & $\mathrm{mg} / \mathrm{L}$ as $\mathrm{F}$ & 0.1 & $\mathrm{I} 232785$ & Fishman and Friedman, 1989 \\
\hline 955 & Silica, dissolved & $\mathrm{mg} / \mathrm{L}$ as $\mathrm{SiO} 2$ & 0.1 & I270085 & Fishman and Friedman, 1989 \\
\hline 1046 & Iron, dissolved & $\mu \mathrm{g} / \mathrm{L}$ & 6.0 & I147287 & Fishman, 1993 \\
\hline 1056 & Manganese, dissolved & $\mu \mathrm{g} / \mathrm{L}$ & 0.6 & I147287 & Fishman, 1993 \\
\hline 39086 & Alkalinity, dissolved & $\mathrm{mg} / \mathrm{L}$ as $\mathrm{CaCO} 3$ & 1.0 & I203085 & Fishman and Friedman, 1989 \\
\hline 70300 & Dissolved solids, residue at $180 \mathrm{oC}$ & $\mathrm{mg} / \mathrm{L}$ & 10.0 & I175085 & Fishman and Friedman, 1989 \\
\hline 90095 & Specific conductance & $\mathrm{ms} / \mathrm{cm}$ & 1.0 & I278185 & Fishman and Friedman, 1989 \\
\hline 90410 & Acid neutralizing capacity & $\mathrm{mg} / \mathrm{L}$ as $\mathrm{CaCO} 3$ & 1.0 & I203085 & Fishman and Friedman, 1989 \\
\hline
\end{tabular}


Dissolved oxygen, specific conductance, temperature, $\mathrm{pH}$, and alkalinity were measured in the field with a Hydrolab $\mathrm{H} 20$ multiparameter probe and individual probes. Escherichia coli were cultured in the field on several occasions using membrane thermotolerant Eshcerichia coli (mTEC) media (Myers and Sylvester, 1997).

Organic stream-water-quality samples were collected as grab samples in baked 1-L amber glass bottles and chilled for shipment to the USGS National Water Quality Laboratory in Denver, Colo. Analyses for specific organic contaminants listed were performed as described in Zaugg and others (2002).

\section{Lake Water Quality}

Synoptic lake-water-quality samples were collected from 12 lake basins in Oakland County during the summers of 2002 and 2003. Sample locations are shown in figure 5. Location information is presented in table 6. Sampling was conducted in late summer, during the period of maximum thermal stratification of lakes. Additional spring samples were collected at several sites. Samples were collected at a depth of three feet below the water surface; three feet above the lake bed; at the thermocline, if present; and throughout the water column. These depths are identical to the stratified sample collected by the USGS in cooperation with the Michigan Department of Environmental Quality Lake Water Quality Assessment program (Minnerick, written communication, 2004). All analyses were conducted by the USGS National Water Quality Laboratory in Denver, Colo. Vertical profile data are presented in table 11. Chemical water-quality data are presented in table 12 .

\section{Ecological Assessment}

During August and September 2003, the USGS conducted ecological assessments of the 14 sites where synoptic water-chemistry samples were collected (figure 4 and table 4). These ecological assessments included habitat assessment, as well as collection and identification of macro-invertebrates. These assessments were carried out in accordance with the Great Lakes and Ecological Assessment Section (GLEAS) Procedure 51 used by the Michigan Department of Environmental Quality (MDEQ; 2002). These data are presented in table 13.

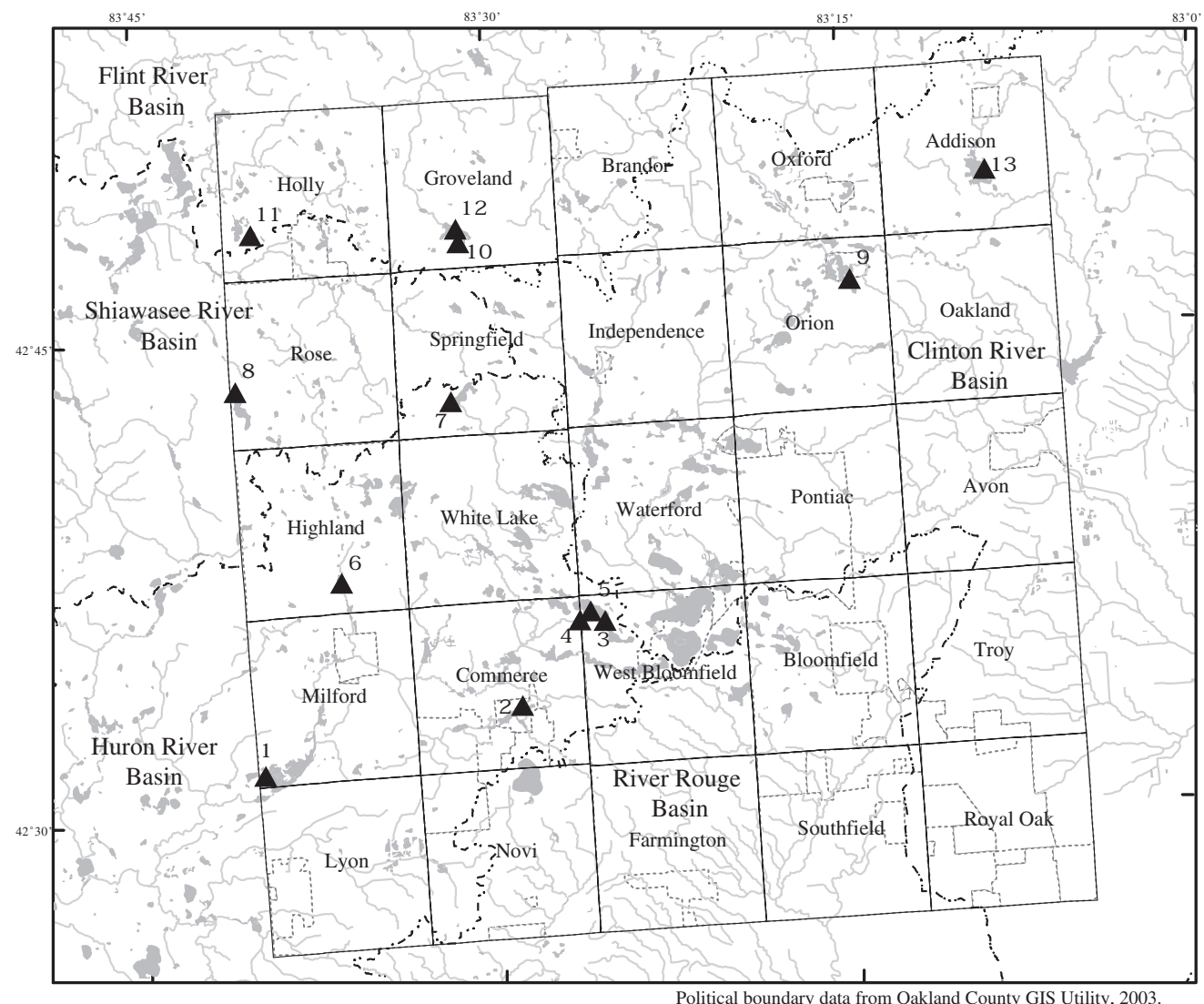

Hydrographic data from USGS 1:100,000 scale Digital Line Graphs.
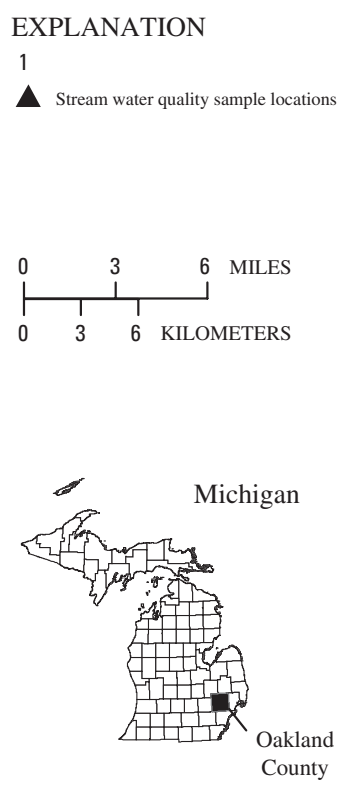

Figure 5. Locations of lake water-quality sampling sites in Oakland County, Michigan.

(Station names can be obtained by cross referencing the number in this figure to table 6 of this report.) 
Table 6. Site number, name, and location of lake water-quality monitoring sites in Oakland County, Mich.

[Reference number corresponds to number on figure 5 of this report]

\begin{tabular}{|c|c|c|c|c|}
\hline $\begin{array}{l}\text { Reference } \\
\text { number }\end{array}$ & Site number & Site name & Latitude & Longitude \\
\hline 1 & 423133083400101 & Kent Lake near New Hudson, Mich. & $42^{\circ} 31^{\prime} 33^{\prime \prime} \mathrm{N}$ & $83^{\circ} 40^{\prime} 01^{\prime \prime} \mathrm{W}$ \\
\hline 2 & 423332083290301 & Wolverine Lake near Oakley Park, Mich. & $42^{\circ} 33^{\prime} 32^{\prime} " \mathrm{~N}$ & $83^{\circ} 29^{\prime} 03^{\prime \prime} \mathrm{W}$ \\
\hline 3 & 423607083252601 & Union Lake at Union Lake, Mich. (southeast basin) & $42^{\circ} 36^{\prime} 07^{\prime \prime} \mathrm{N}$ & $83^{\circ} 25^{\prime} 26^{\prime \prime} \mathrm{W}$ \\
\hline 4 & 423609083263001 & Union Lake at Union Lake, Mich. (southwest basin) & $42^{\circ} 36^{\prime} 09^{\prime \prime} \mathrm{N}$ & $83^{\circ} 26^{\prime} 30^{\prime \prime} \mathrm{W}$ \\
\hline 5 & 423626083260201 & Union Lake at Union Lake, Mich. (central basin) & $42^{\circ} 36^{\prime} 26^{\prime \prime} \mathrm{N}$ & $83^{\circ} 26^{\prime} 02^{\prime} \mathrm{W}$ \\
\hline 6 & 423730083363401 & Lower Pettibone Lake near Highland, Mich. & $42^{\circ} 37^{\prime} 30^{\prime \prime} \mathrm{N}$ & $83^{\circ} 36^{\prime} 34^{\prime \prime} \mathrm{W}$ \\
\hline 7 & 424306083314201 & Big Lake at Andersonville, Mich. & $42^{\circ} 43^{\prime} 06^{\prime \prime} \mathrm{N}$ & $83^{\circ} 31^{\prime} 42^{\prime} " \mathrm{~W}$ \\
\hline 8 & 424335083405201 & Tipsico Lake near Rose Center, Mich. & $42^{\circ} 43^{\prime} 35^{\prime \prime} \mathrm{N}$ & $83^{\circ} 40^{\prime} 52^{\prime} \mathrm{W}$ \\
\hline 9 & 424634083143601 & Lake Orion near Lake Orion, Mich. & $42^{\circ} 46^{\prime} 34^{\prime \prime} \mathrm{N}$ & $83^{\circ} 14^{\prime} 36^{\prime \prime} \mathrm{W}$ \\
\hline 10 & 424804083311301 & Valley Lake near Davisburg, Mich. & $42^{\circ} 48^{\prime} 04^{\prime \prime} \mathrm{N}$ & $83^{\circ} 31^{\prime} 13^{\prime \prime} \mathrm{W}$ \\
\hline 11 & 424828083400201 & Dickinson Lake near Holly, Mich. & $42^{\circ} 48^{\prime} 28^{\prime \prime} \mathrm{N}$ & $83^{\circ} 40^{\prime} 02^{\prime} \mathrm{W}$ \\
\hline 12 & 424830083311801 & Heron Lake near Holly, Mich. & $42^{\circ} 48^{\prime} 30^{\prime \prime} \mathrm{N}$ & $83^{\circ} 31^{\prime} 18^{\prime \prime} \mathrm{W}$ \\
\hline 13 & 424952083084301 & Lakeville Lake near Lakeville, Mich. & $42^{\circ} 49^{\prime} 52^{\prime \prime} \mathrm{N}$ & $83^{\circ} 08^{\prime} 43^{\prime \prime} \mathrm{W}$ \\
\hline
\end{tabular}

\section{References Cited}

Blumer, S.P., Behrendt, T.E., Ellis, J.M., Minnerick, R.J., LeuVoy, R.L., and Whited, C.R., 2002, Water Resource Data - Michigan - Water Year 2001: U.S. Geological Survey Water-Data report MI-01-1, 462 p.

Blumer, S.P., Behrendt, T.E., Ellis, J.M., Minnerick, R.J., LeuVoy, R.L., and Whited, C.R., 2003, Water Resource Data - Michigan - Water Year 2002: U.S. Geological Survey Water-Data report MI-02-1, 516 p.

Blumer, S.P., Behrendt, T.E., Ellis, J.M., Minnerick, R.J., LeuVoy, R.L., and Whited, C.R., 2004, Water Resource Data - Michigan - Water Year 2003: U.S. Geological Survey Water-Data report MI-03-1, $526 \mathrm{p}$.

Fishman, M.J., 1993, Methods of analysis of the U.S. Geological Survey National Water Quality Laboratory - Determination of inorganic and organic constituents in water and fluvial sediments: U.S. Geological Survey Open-File Report 93-125, 273 p.

Fishman, M.J., and Friedman, L.C., 1989, Methods for determination of inorganic substances in water and fluvial sediments: U.S. Geological Survey Techniques of Water Resources Investigations, book 5, chap. A1, 454 p.

MDEQ, 2002, Qualitative Biological and habitat survey protocols for wadable streams and rivers: http://www.deq. state. mi.us/documents/deq-swq-gleas-proc51.pdf
Minnerick, R.J., 2004, Supervisory Hydrologic Technician, U.S. Geological Survey, Grayling, Michigan. Field protocol for sampling of inland lakes in Michigan.

Myers, D.N., and Sylvester, M.D., 1997. National Field Manual for the collection of water-quality data - Biological indicators: U.S. Geological Survey Techniques of WaterResources Investigations, book 9, chap. A7, 38 p.

Patton, C.J., and Truitt, E.P., 1992, Methods of analysis of the U.S. Geological Survey National Water Quality Laboratory - Determination of total phosphorus by a Kjeldahl digestion method and an automated colorometric finish that includes dialysis: U.S. Geological Survey Open-File Report 92-146, $39 \mathrm{p}$.

Patton, C.J., and Truitt, E.P., 2000, Methods of analysis by the U.S. Geological Survey National Water Quality Laboratory - Determination of ammonium plus organic nitrogen by a Kjeldahl digestion method and an automated photometric finish that includes digest cleanup by gas diffusion: U.S. Geolocial Survey Open-File Report 00-170, 31 p.

Rantz, S.E., and others, 1982, Measurement and computation of streamflow, v. 1, measurement of stage and discharge: U. S. Geological Survey Water Supply Paper 2175, 284 p.

Wagner, R.J., Mattraw, H.C., Ritz, G.F., and Smith, B.A., 2000. Guidelines and procedures for continuous waterquality monitors: site selection, field calibration, record computation, and reporting: U. S. Geological Survey WaterResources Investigations Report 00-4252, 26 p. 
Wilde, F.D., Radtke, D.B., Gibs, Jacob, and Iwatsubo, R.T., eds., September 1999, Collection of water samples: U.S. Geological Survey Techniques of Water-Resources Investigations, book 9, chap. A4, accessed Oct. 12, 2004 at http:// pubs.water.usgs.gov/twri9A4/

Wilde, F.D., Radtke, D.B., Gibs, Jacob, and Iwatsubo, R.T., eds., March 2003, Cleaning of Equipment for water sampling (ver. 1.2): U.S. Geological Survey Techniques of Water-Resources Investigations, book 9, chap. A3, accessed Oct. 12, 2004 at http://pubs.water.usgs.gov/twri9A3/

Wilde, F.D., Radtke, D.B., Gibs, Jacob, and Iwatsubo, R.T., eds., April 2004, Processing of water samples (version 2.1): U.S. Geological Survey Techniques of Water-Resources Investigations, book 9, chap. A5, accessed Oct. 12, 2004 at http://pubs.water.usgs.gov/twri9A5/

U.S. Environmental Protection Agency, 1994, Methods for the determination of inorganic substances in environmental samples: Available from National Technical Information Service as PB94-120821, 169 p.

Zaugg, S.D., Smith, S.G., Schroeder, M.P., Barber, L.B., and Burkhardt, M.R., 2002, Methods of analysis by the U.S. Geological Survey National Water Quality Laboratory--Determination of wastewater compounds by polystyrenedivinylbenzene solid-phase extraction and capillary-column gas chromatography/mass spectrometry: U.S. Geological Survey Water-Resources Investigations Report 01-4186, 37 p. 
Table 7. Daily mean and maximum depth, in feet, of water below land surface in four monitoring wells in Oakland County, Michigan, June 2001 through March 2004

$[--$, no data $]$

\begin{tabular}{|c|c|c|c|c|c|c|c|c|c|c|}
\hline \multirow[b]{2}{*}{ Year } & \multirow[b]{2}{*}{ Month } & \multirow[b]{2}{*}{ Day } & \multicolumn{2}{|c|}{$\begin{array}{c}\text { Holly State Rec. Area } \\
425116083321501\end{array}$} & \multicolumn{2}{|c|}{$\begin{array}{l}\text { Proud Lake State Rec. Area } \\
\quad 423423083324001\end{array}$} & \multicolumn{2}{|c|}{$\begin{array}{l}\text { Fish Lake Road } \\
424109083384301\end{array}$} & \multicolumn{2}{|c|}{$\begin{array}{l}\text { Teggerdine Road } \\
424133083293101\end{array}$} \\
\hline & & & Mean & Maximum & Mean & Maximum & Mean & Maximum & Mean & Maximum \\
\hline 2001 & 6 & 1 & -- & -- & -- & -- & 34.71 & 34.72 & -- & -- \\
\hline 2001 & 6 & 2 & -- & -- & -- & -- & 34.69 & 34.70 & -- & -- \\
\hline 2001 & 6 & 3 & -- & -- & -- & -- & 34.69 & 34.69 & -- & -- \\
\hline 2001 & 6 & 4 & -- & -- & -- & -- & 34.68 & 34.69 & -- & -- \\
\hline 2001 & 6 & 5 & -- & -- & -- & -- & 34.66 & 34.68 & -- & -- \\
\hline 2001 & 6 & 6 & -- & -- & -- & -- & 34.65 & 34.66 & -- & -- \\
\hline 2001 & 6 & 7 & -- & -- & -- & -- & 34.64 & 34.65 & -- & -- \\
\hline 2001 & 6 & 8 & -- & -- & -- & -- & 34.63 & 34.64 & 28.72 & 28.76 \\
\hline 2001 & 6 & 9 & -- & -- & -- & -- & 34.62 & 34.62 & 28.74 & 28.76 \\
\hline 2001 & 6 & 10 & -- & -- & -- & -- & 34.61 & 34.61 & 28.75 & 28.76 \\
\hline 2001 & 6 & 11 & -- & -- & -- & -- & 34.60 & 34.60 & 28.75 & 28.76 \\
\hline 2001 & 6 & 12 & -- & -- & -- & -- & 34.59 & 34.60 & 28.77 & 28.78 \\
\hline 2001 & 6 & 13 & -- & -- & -- & -- & 34.58 & 34.59 & 28.80 & 28.81 \\
\hline 2001 & 6 & 14 & 25.98 & 26.02 & -- & -- & 34.58 & 34.58 & 28.83 & 28.85 \\
\hline 2001 & 6 & 15 & 26.01 & 26.02 & -- & -- & 34.57 & 34.58 & 28.85 & 28.87 \\
\hline 2001 & 6 & 16 & 26.04 & 26.07 & -- & -- & 34.58 & 34.58 & 28.88 & 28.89 \\
\hline 2001 & 6 & 17 & 26.09 & 26.16 & -- & -- & 34.57 & 34.58 & 28.91 & 28.93 \\
\hline 2001 & 6 & 18 & 26.11 & 26.13 & -- & -- & 34.56 & 34.57 & 28.93 & 28.95 \\
\hline 2001 & 6 & 19 & 26.13 & 26.19 & -- & -- & 34.56 & 34.56 & 28.95 & 28.97 \\
\hline 2001 & 6 & 20 & 26.18 & 26.21 & -- & -- & 34.55 & 34.56 & 28.98 & 29.00 \\
\hline 2001 & 6 & 21 & 26.16 & 26.19 & -- & -- & 34.55 & 34.55 & 29.00 & 29.01 \\
\hline 2001 & 6 & 22 & 26.02 & 26.06 & -- & -- & 34.54 & 34.54 & 28.94 & 28.95 \\
\hline 2001 & 6 & 23 & 26.02 & 26.04 & -- & -- & 34.53 & 34.54 & 28.95 & 28.98 \\
\hline 2001 & 6 & 24 & 26.07 & 26.12 & -- & -- & 34.53 & 34.53 & 28.99 & 29.01 \\
\hline 2001 & 6 & 25 & 26.12 & 26.16 & -- & -- & 34.52 & 34.52 & 29.02 & 29.04 \\
\hline 2001 & 6 & 26 & 26.15 & 26.18 & -- & -- & 34.51 & 34.52 & 29.05 & 29.07 \\
\hline 2001 & 6 & 27 & 26.19 & 26.22 & -- & -- & 34.50 & 34.51 & 29.07 & 29.09 \\
\hline 2001 & 6 & 28 & 26.23 & 26.25 & -- & -- & 34.49 & 34.50 & 29.09 & 29.10 \\
\hline 2001 & 6 & 29 & 26.26 & 26.32 & -- & -- & 34.48 & 34.49 & 29.11 & 29.13 \\
\hline 2001 & 6 & 30 & 26.32 & 26.36 & -- & -- & 34.47 & 34.48 & 29.13 & 29.16 \\
\hline 2001 & 7 & 1 & 26.38 & 26.43 & -- & -- & 34.47 & 34.47 & 29.18 & 29.22 \\
\hline 2001 & 7 & 2 & 26.42 & 26.44 & -- & -- & 34.46 & 34.47 & 29.22 & 29.23 \\
\hline 2001 & 7 & 3 & 26.41 & 26.43 & -- & -- & 34.45 & 34.46 & 29.23 & 29.26 \\
\hline 2001 & 7 & 4 & 26.44 & 26.50 & -- & -- & 34.44 & 34.45 & 29.22 & 29.24 \\
\hline 2001 & 7 & 5 & 26.50 & 26.54 & -- & -- & 34.44 & 34.44 & 29.25 & 29.27 \\
\hline 2001 & 7 & 6 & 26.54 & 26.56 & -- & -- & 34.43 & 34.44 & 29.28 & 29.30 \\
\hline 2001 & 7 & 7 & 26.55 & 26.57 & -- & -- & 34.43 & 34.43 & 29.29 & 29.32 \\
\hline 2001 & 7 & 8 & 26.59 & 26.64 & -- & -- & 34.42 & 34.42 & 29.30 & 29.33 \\
\hline 2001 & 7 & 9 & 26.63 & 26.68 & -- & -- & 34.41 & 34.42 & 29.33 & 29.35 \\
\hline 2001 & 7 & 10 & 26.67 & 26.72 & -- & -- & 34.41 & 34.42 & 29.35 & 29.40 \\
\hline 2001 & 7 & 11 & 26.73 & 26.78 & -- & -- & 34.41 & 34.42 & 29.40 & 29.42 \\
\hline 2001 & 7 & 12 & 26.80 & 26.87 & -- & -- & 34.42 & 34.42 & 29.44 & 29.46 \\
\hline 2001 & 7 & 13 & 26.85 & 26.88 & -- & -- & 34.42 & 34.42 & 29.46 & 29.48 \\
\hline 2001 & 7 & 14 & 26.87 & 26.88 & -- & -- & 34.41 & 34.42 & 29.47 & 29.49 \\
\hline 2001 & 7 & 15 & 26.91 & 26.98 & -- & -- & 34.41 & 34.41 & 29.51 & 29.53 \\
\hline 2001 & 7 & 16 & 26.96 & 27.02 & -- & -- & 34.41 & 34.41 & 29.54 & 29.57 \\
\hline
\end{tabular}


Table 7. Daily mean and maximum depth, in feet, of water below land surface in four monitoring wells in Oakland County, Michigan, June 2001 through March 2004 - continued $[--$, no data $]$

\begin{tabular}{|c|c|c|c|c|c|c|c|c|c|c|}
\hline \multirow[b]{2}{*}{ Year } & \multirow[b]{2}{*}{ Month } & \multirow[b]{2}{*}{ Day } & \multicolumn{2}{|c|}{$\begin{array}{c}\text { Holly State Rec. Area } \\
425116083321501\end{array}$} & \multicolumn{2}{|c|}{$\begin{array}{c}\text { Proud Lake State Rec. Area } \\
423423083324001\end{array}$} & \multicolumn{2}{|c|}{$\begin{array}{l}\text { Fish Lake Road } \\
424109083384301\end{array}$} & \multicolumn{2}{|c|}{$\begin{array}{l}\text { Teggerdine Road } \\
424133083293101\end{array}$} \\
\hline & & & Mean & Maximum & Mean & Maximum & Mean & Maximum & Mean & Maximum \\
\hline 2001 & 7 & 17 & 26.97 & 26.99 & -- & -- & 34.41 & 34.41 & 29.57 & 29.60 \\
\hline 2001 & 7 & 18 & 26.96 & 26.97 & -- & -- & 34.42 & 34.42 & 29.60 & 29.63 \\
\hline 2001 & 7 & 19 & 26.97 & 27.03 & -- & -- & 34.42 & 34.43 & 29.63 & 29.66 \\
\hline 2001 & 7 & 20 & 27.00 & 27.07 & -- & -- & 34.43 & 34.43 & 29.67 & 29.69 \\
\hline 2001 & 7 & 21 & 27.02 & 27.04 & -- & -- & 34.42 & 34.43 & 29.69 & 29.70 \\
\hline 2001 & 7 & 22 & 27.05 & 27.08 & -- & -- & 34.42 & 34.42 & 29.71 & 29.73 \\
\hline 2001 & 7 & 23 & 27.07 & 27.13 & -- & -- & 34.42 & 34.42 & 29.74 & 29.75 \\
\hline 2001 & 7 & 24 & 27.11 & 27.15 & -- & -- & 34.43 & 34.43 & 29.76 & 29.79 \\
\hline 2001 & 7 & 25 & 27.15 & 27.16 & -- & -- & 34.44 & 34.44 & 29.80 & 29.80 \\
\hline 2001 & 7 & 26 & 27.17 & 27.24 & -- & -- & 34.45 & 34.46 & 29.81 & 29.83 \\
\hline 2001 & 7 & 27 & 27.18 & 27.20 & -- & -- & 34.46 & 34.47 & 29.83 & 29.84 \\
\hline 2001 & 7 & 28 & 27.19 & 27.21 & -- & -- & 34.46 & 34.46 & 29.84 & 29.85 \\
\hline 2001 & 7 & 29 & 27.22 & 27.25 & -- & -- & 34.45 & 34.46 & 29.86 & 29.88 \\
\hline 2001 & 7 & 30 & 27.26 & 27.28 & -- & -- & 34.46 & 34.47 & 29.88 & 29.89 \\
\hline 2001 & 7 & 31 & 27.29 & 27.34 & -- & -- & 34.47 & 34.48 & 29.90 & 29.91 \\
\hline 2001 & 8 & 1 & 27.34 & 27.41 & -- & -- & 34.48 & 34.48 & 29.93 & 29.94 \\
\hline 2001 & 8 & 2 & 27.36 & 27.40 & -- & -- & 34.47 & 34.48 & 29.94 & 29.95 \\
\hline 2001 & 8 & 3 & 27.38 & 27.41 & -- & -- & 34.47 & 34.47 & 29.95 & 29.96 \\
\hline 2001 & 8 & 4 & 27.43 & 27.47 & -- & -- & 34.47 & 34.47 & 29.97 & 29.99 \\
\hline 2001 & 8 & 5 & 27.48 & 27.53 & -- & -- & 34.48 & 34.48 & 30.01 & 30.02 \\
\hline 2001 & 8 & 6 & 27.51 & 27.54 & -- & -- & 34.48 & 34.48 & 30.03 & 30.03 \\
\hline 2001 & 8 & 7 & 27.53 & 27.55 & -- & -- & 34.48 & 34.48 & 30.06 & 30.07 \\
\hline 2001 & 8 & 8 & 27.56 & 27.62 & -- & -- & 34.48 & 34.49 & 30.08 & 30.09 \\
\hline 2001 & 8 & 9 & 27.60 & 27.64 & -- & -- & 34.48 & 34.49 & 30.11 & 30.12 \\
\hline 2001 & 8 & 10 & 27.63 & 27.66 & -- & -- & 34.50 & 34.51 & 30.14 & 30.15 \\
\hline 2001 & 8 & 11 & 27.66 & 27.68 & -- & -- & 34.51 & 34.51 & 30.16 & 30.16 \\
\hline 2001 & 8 & 12 & 27.69 & 27.76 & -- & -- & 34.51 & 34.52 & 30.19 & 30.19 \\
\hline 2001 & 8 & 13 & 27.71 & 27.75 & -- & -- & 34.52 & 34.53 & 30.21 & 30.22 \\
\hline 2001 & 8 & 14 & 27.74 & 27.79 & -- & -- & 34.54 & 34.54 & 30.24 & 30.23 \\
\hline 2001 & 8 & 15 & 27.75 & 27.78 & -- & -- & 34.54 & 34.54 & 30.25 & 30.24 \\
\hline 2001 & 8 & 16 & 27.74 & 27.76 & -- & -- & 34.54 & 34.55 & 30.24 & 30.25 \\
\hline 2001 & 8 & 17 & 27.73 & 27.74 & -- & -- & 34.55 & 34.56 & 30.22 & 30.21 \\
\hline 2001 & 8 & 18 & 27.69 & 27.71 & 5.61 & 5.65 & 34.56 & 34.56 & 30.20 & 30.20 \\
\hline 2001 & 8 & 19 & 27.65 & 27.66 & 5.52 & 5.56 & 34.55 & 34.56 & 30.15 & 30.18 \\
\hline 2001 & 8 & 20 & 27.63 & 27.64 & 5.49 & 5.50 & 34.57 & 34.58 & 30.12 & 30.12 \\
\hline 2001 & 8 & 21 & 27.62 & 27.62 & 5.50 & 5.52 & 34.58 & 34.59 & 30.11 & 30.11 \\
\hline 2001 & 8 & 22 & 27.61 & 27.63 & 5.48 & 5.52 & 34.59 & 34.59 & 30.10 & 30.11 \\
\hline 2001 & 8 & 23 & 27.59 & 27.61 & 5.47 & 5.48 & 34.59 & 34.60 & 30.07 & 30.07 \\
\hline 2001 & 8 & 24 & 27.60 & 27.63 & 5.48 & 5.49 & 34.61 & 34.61 & 30.07 & 30.06 \\
\hline 2001 & 8 & 25 & 27.60 & 27.61 & 5.49 & 5.49 & 34.61 & 34.61 & 30.05 & 30.06 \\
\hline 2001 & 8 & 26 & 27.61 & 27.64 & 5.48 & 5.50 & 34.62 & 34.62 & 30.04 & 30.04 \\
\hline 2001 & 8 & 27 & 27.63 & 27.65 & 5.48 & 5.49 & 34.62 & 34.63 & 30.05 & 30.04 \\
\hline 2001 & 8 & 28 & 27.67 & 27.70 & 5.51 & 5.54 & 34.64 & 34.65 & 30.07 & 30.10 \\
\hline 2001 & 8 & 29 & 27.70 & 27.72 & 5.55 & 5.56 & 34.65 & 34.65 & 30.11 & 30.12 \\
\hline 2001 & 8 & 30 & 27.72 & 27.74 & 5.56 & 5.57 & 34.65 & 34.66 & 30.12 & 30.14 \\
\hline 2001 & 8 & 31 & 27.76 & 27.79 & 5.59 & 5.63 & 34.66 & 34.67 & 30.15 & 30.19 \\
\hline
\end{tabular}


Table 7. Daily mean and maximum depth, in feet, of water below land surface in four monitoring wells in Oakland County, Michigan, June 2001 through March 2004 - continued $[--$, no data $]$

\begin{tabular}{|c|c|c|c|c|c|c|c|c|c|c|}
\hline \multirow[b]{2}{*}{ Year } & \multirow[b]{2}{*}{ Month } & \multirow[b]{2}{*}{ Day } & \multicolumn{2}{|c|}{$\begin{array}{c}\text { Holly State Rec. Area } \\
425116083321501\end{array}$} & \multicolumn{2}{|c|}{$\begin{array}{c}\text { Proud Lake State Rec. Area } \\
423423083324001\end{array}$} & \multicolumn{2}{|c|}{$\begin{array}{l}\text { Fish Lake Road } \\
424109083384301\end{array}$} & \multicolumn{2}{|c|}{$\begin{array}{l}\text { Teggerdine Road } \\
424133083293101\end{array}$} \\
\hline & & & Mean & Maximum & Mean & Maximum & Mean & Maximum & Mean & Maximum \\
\hline 2001 & 9 & 1 & 27.79 & 27.81 & 5.65 & 5.66 & 34.68 & 34.68 & 30.20 & 30.23 \\
\hline 2001 & 9 & 2 & -- & -- & 5.66 & 5.68 & 34.69 & 34.69 & 30.23 & 30.24 \\
\hline 2001 & 9 & 3 & -- & -- & 5.68 & 5.71 & 34.69 & 34.69 & 30.24 & 30.27 \\
\hline 2001 & 9 & 4 & -- & -- & -- & 5.80 & 34.70 & 34.71 & 30.28 & 30.30 \\
\hline 2001 & 9 & 5 & 27.86 & 27.89 & 5.74 & 5.82 & 34.71 & 34.72 & 30.31 & 30.32 \\
\hline 2001 & 9 & 6 & 27.88 & 27.90 & 5.75 & 5.85 & 34.72 & 34.72 & 30.31 & 30.33 \\
\hline 2001 & 9 & 7 & 27.90 & 27.92 & 5.77 & 5.79 & 34.71 & 34.72 & 30.33 & 30.34 \\
\hline 2001 & 9 & 8 & 27.94 & 27.99 & 5.79 & 5.80 & 34.72 & 34.73 & 30.34 & 30.36 \\
\hline 2001 & 9 & 9 & 27.87 & 27.92 & 5.73 & 5.77 & 34.73 & 34.73 & 30.33 & 30.36 \\
\hline 2001 & 9 & 10 & 27.82 & 27.83 & 5.70 & 5.93 & 34.74 & 34.75 & 30.31 & 30.33 \\
\hline 2001 & 9 & 11 & 27.81 & 27.83 & 5.69 & 5.76 & 34.75 & 34.75 & 30.31 & 30.33 \\
\hline 2001 & 9 & 12 & 27.79 & 27.81 & 5.71 & 5.73 & 34.75 & 34.76 & 30.31 & 30.32 \\
\hline 2001 & 9 & 13 & 27.82 & 27.85 & 5.74 & 5.78 & 34.76 & 34.77 & 30.33 & 30.36 \\
\hline 2001 & 9 & 14 & 27.83 & 27.85 & 5.75 & 5.78 & 34.77 & 34.78 & 30.34 & 30.36 \\
\hline 2001 & 9 & 15 & 27.83 & 27.86 & 5.75 & 5.78 & 34.78 & 34.78 & 30.33 & 30.34 \\
\hline 2001 & 9 & 16 & 27.84 & 27.86 & 5.79 & 5.86 & 34.79 & 34.79 & 30.32 & 30.35 \\
\hline 2001 & 9 & 17 & 27.85 & 27.86 & 5.80 & 5.87 & 34.79 & 34.80 & 30.34 & 30.36 \\
\hline 2001 & 9 & 18 & 27.86 & 27.88 & 5.80 & 5.84 & 34.80 & 34.81 & 30.36 & 30.38 \\
\hline 2001 & 9 & 19 & 27.82 & 27.88 & 5.72 & 5.82 & 34.81 & 34.81 & 30.31 & 30.38 \\
\hline 2001 & 9 & 20 & 27.79 & 27.80 & 5.69 & 5.74 & 34.82 & 34.83 & 30.25 & 30.27 \\
\hline 2001 & 9 & 21 & 27.75 & 27.79 & 5.65 & 5.69 & 34.83 & 34.84 & 30.23 & 30.25 \\
\hline 2001 & 9 & 22 & 27.68 & 27.71 & 5.65 & 5.68 & 34.84 & 34.85 & 30.20 & 30.22 \\
\hline 2001 & 9 & 23 & 27.64 & 27.66 & 5.62 & 5.67 & 34.84 & 34.85 & 30.16 & 30.19 \\
\hline 2001 & 9 & 24 & 27.63 & 27.64 & 5.59 & 5.61 & 34.85 & 34.85 & 30.14 & 30.15 \\
\hline 2001 & 9 & 25 & 27.59 & 27.62 & 5.57 & 5.61 & 34.84 & 34.84 & 30.12 & 30.14 \\
\hline 2001 & 9 & 26 & 27.53 & 27.56 & 5.54 & 5.56 & 34.84 & 34.84 & 30.07 & 30.11 \\
\hline 2001 & 9 & 27 & 27.54 & 27.54 & 5.55 & 5.56 & 34.85 & 34.85 & 30.07 & 30.09 \\
\hline 2001 & 9 & 28 & 27.54 & 27.57 & 5.55 & 5.61 & 34.85 & 34.86 & 30.08 & 30.09 \\
\hline 2001 & 9 & 29 & 27.55 & 27.58 & 5.56 & 5.58 & 34.86 & 34.86 & 30.09 & 30.10 \\
\hline 2001 & 9 & 30 & 27.55 & 27.57 & 5.56 & 5.58 & 34.86 & 34.86 & 30.08 & 30.10 \\
\hline 2001 & 10 & 1 & 27.53 & 27.54 & 5.56 & 5.61 & 34.85 & 34.85 & 30.07 & 30.08 \\
\hline 2001 & 10 & 2 & 27.55 & 27.56 & 5.57 & 5.61 & 34.86 & 34.86 & 30.07 & 30.08 \\
\hline 2001 & 10 & 3 & 27.56 & 27.59 & 5.58 & 5.61 & 34.86 & 34.87 & 30.07 & 30.08 \\
\hline 2001 & 10 & 4 & 27.60 & 27.61 & 5.60 & 5.64 & 34.87 & 34.88 & 30.10 & 30.12 \\
\hline 2001 & 10 & 5 & 27.54 & 27.60 & 5.51 & 5.57 & 34.87 & 34.88 & 30.05 & 30.09 \\
\hline 2001 & 10 & 6 & 27.46 & 27.48 & 5.48 & 5.51 & 34.88 & 34.89 & 30.01 & 30.03 \\
\hline 2001 & 10 & 7 & 27.46 & 27.47 & 5.50 & 5.52 & 34.89 & 34.89 & 30.00 & 30.03 \\
\hline 2001 & 10 & 8 & 27.46 & 27.49 & 5.49 & 5.54 & 34.89 & 34.90 & 30.01 & 30.04 \\
\hline 2001 & 10 & 9 & 27.44 & 27.46 & 5.47 & 5.51 & 34.89 & 34.89 & 29.99 & 30.00 \\
\hline 2001 & 10 & 10 & 27.45 & 27.47 & 5.47 & 5.52 & 34.88 & 34.89 & 29.97 & 29.99 \\
\hline 2001 & 10 & 11 & 27.45 & 27.47 & 5.45 & 5.48 & 34.88 & 34.89 & 29.96 & 29.98 \\
\hline 2001 & 10 & 12 & 27.40 & 27.45 & 5.39 & 5.43 & 34.88 & 34.89 & 29.90 & 29.94 \\
\hline 2001 & 10 & 13 & 27.36 & 27.40 & 5.37 & 5.40 & 34.87 & 34.89 & 29.85 & 29.88 \\
\hline 2001 & 10 & 14 & 27.29 & 27.33 & 5.34 & 5.38 & 34.86 & 34.87 & 29.79 & 29.83 \\
\hline 2001 & 10 & 15 & 27.28 & 27.30 & 5.34 & 5.37 & 34.88 & 34.88 & 29.77 & 29.78 \\
\hline 2001 & 10 & 16 & 27.18 & 27.29 & 5.24 & 5.34 & 34.85 & 34.87 & 29.68 & 29.77 \\
\hline
\end{tabular}


Table 7. Daily mean and maximum depth, in feet, of water below land surface in four monitoring wells in Oakland County, Michigan, June 2001 through March 2004 - continued $[--$, no data $]$

\begin{tabular}{|c|c|c|c|c|c|c|c|c|c|c|}
\hline \multirow[b]{2}{*}{ Year } & \multirow[b]{2}{*}{ Month } & \multirow[b]{2}{*}{ Day } & \multicolumn{2}{|c|}{$\begin{array}{c}\text { Holly State Rec. Area } \\
425116083321501\end{array}$} & \multicolumn{2}{|c|}{$\begin{array}{c}\text { Proud Lake State Rec. Area } \\
423423083324001\end{array}$} & \multicolumn{2}{|c|}{$\begin{array}{c}\text { Fish Lake Road } \\
424109083384301\end{array}$} & \multicolumn{2}{|c|}{$\begin{array}{l}\text { Teggerdine Road } \\
424133083293101\end{array}$} \\
\hline & & & Mean & Maximum & Mean & Maximum & Mean & Maximum & Mean & Maximum \\
\hline 2001 & 10 & 17 & 27.10 & 27.12 & 5.15 & 5.18 & 34.82 & 34.85 & 29.52 & 29.59 \\
\hline 2001 & 10 & 18 & 27.06 & 27.10 & 5.08 & 5.14 & 34.76 & 34.79 & 29.45 & 29.50 \\
\hline 2001 & 10 & 19 & 27.04 & 27.05 & 5.04 & 5.12 & 34.71 & 34.73 & 29.41 & 29.42 \\
\hline 2001 & 10 & 20 & 27.05 & 27.05 & 5.04 & 5.06 & 34.67 & 34.69 & 29.39 & 29.42 \\
\hline 2001 & 10 & 21 & 27.04 & 27.07 & 5.04 & 5.10 & 34.65 & 34.66 & 29.40 & 29.43 \\
\hline 2001 & 10 & 22 & 27.04 & 27.05 & 5.02 & 5.08 & 34.62 & 34.63 & 29.40 & 29.43 \\
\hline 2001 & 10 & 23 & 26.99 & 27.01 & 4.98 & 5.03 & 34.59 & 34.60 & -- & -- \\
\hline 2001 & 10 & 24 & -- & -- & 4.97 & 5.01 & 34.57 & 34.58 & -- & -- \\
\hline 2001 & 10 & 25 & -- & -- & 4.93 & 4.97 & 34.56 & 34.57 & -- & -- \\
\hline 2001 & 10 & 26 & -- & -- & 4.93 & 4.96 & 34.56 & 34.56 & -- & -- \\
\hline 2001 & 10 & 27 & -- & -- & 4.94 & 4.99 & 34.56 & 34.56 & -- & -- \\
\hline 2001 & 10 & 28 & -- & -- & 4.93 & 4.96 & 34.54 & 34.55 & -- & -- \\
\hline 2001 & 10 & 29 & -- & -- & 4.92 & 4.99 & 34.53 & 34.54 & -- & -- \\
\hline 2001 & 10 & 30 & -- & -- & 4.92 & 4.98 & 34.52 & 34.53 & -- & -- \\
\hline 2001 & 10 & 31 & -- & -- & 4.88 & 4.91 & 34.51 & 34.52 & -- & -- \\
\hline 2001 & 11 & 1 & -- & -- & 4.89 & 5.02 & 34.50 & 34.50 & -- & -- \\
\hline 2001 & 11 & 2 & 26.91 & 26.94 & 4.87 & 4.90 & 34.49 & 34.50 & 29.34 & 29.38 \\
\hline 2001 & 11 & 3 & 26.90 & 26.92 & 4.88 & 4.92 & 34.50 & 34.51 & 29.34 & 29.35 \\
\hline 2001 & 11 & 4 & 26.88 & 26.92 & 4.87 & 4.93 & 34.49 & 34.49 & 29.34 & 29.35 \\
\hline 2001 & 11 & 5 & 26.89 & 26.91 & 4.88 & 4.94 & 34.48 & 34.49 & 29.35 & 29.36 \\
\hline 2001 & 11 & 6 & 26.88 & 26.90 & 4.87 & 4.93 & 34.47 & 34.48 & 29.35 & 29.36 \\
\hline 2001 & 11 & 7 & 26.88 & 26.90 & 4.87 & 4.92 & 34.47 & 34.48 & 29.36 & 29.36 \\
\hline 2001 & 11 & 8 & 26.87 & 26.92 & 4.87 & 4.90 & 34.47 & 34.49 & 29.36 & 29.40 \\
\hline 2001 & 11 & 9 & 26.89 & 26.92 & 4.88 & 4.91 & 34.48 & 34.49 & 29.39 & 29.40 \\
\hline 2001 & 11 & 10 & 26.86 & 26.91 & 4.86 & 4.91 & 34.47 & 34.49 & 29.37 & 29.40 \\
\hline 2001 & 11 & 11 & 26.92 & 26.94 & 4.90 & 4.97 & 34.50 & 34.51 & 29.43 & 29.45 \\
\hline 2001 & 11 & 12 & 26.92 & 26.94 & 4.88 & 4.95 & 34.51 & 34.51 & 29.45 & 29.46 \\
\hline 2001 & 11 & 13 & 26.89 & 26.91 & 4.86 & 4.92 & 34.50 & 34.51 & 29.44 & 29.46 \\
\hline 2001 & 11 & 14 & 26.89 & 26.90 & 4.86 & 4.90 & 34.50 & 34.51 & 29.44 & 29.44 \\
\hline 2001 & 11 & 15 & 26.83 & 26.88 & 4.85 & 4.96 & 34.51 & 34.51 & 29.42 & 29.44 \\
\hline 2001 & 11 & 16 & 26.83 & 26.85 & 4.85 & 4.88 & 34.53 & 34.54 & 29.43 & 29.46 \\
\hline 2001 & 11 & 17 & 26.84 & 26.86 & 4.85 & 4.88 & 34.54 & 34.55 & 29.46 & 29.47 \\
\hline 2001 & 11 & 18 & 26.81 & 26.83 & 4.84 & 4.86 & 34.54 & 34.55 & 29.45 & 29.46 \\
\hline 2001 & 11 & 19 & 26.80 & 26.84 & 4.84 & 4.90 & 34.54 & 34.56 & 29.42 & 29.45 \\
\hline 2001 & 11 & 20 & 26.83 & 26.84 & 4.85 & 4.90 & 34.57 & 34.57 & 29.45 & 29.48 \\
\hline 2001 & 11 & 21 & 26.80 & 26.83 & 4.84 & 4.89 & 34.57 & 34.58 & 29.43 & 29.46 \\
\hline 2001 & 11 & 22 & 26.83 & 26.84 & 4.85 & 4.86 & 34.59 & 34.59 & 29.46 & 29.50 \\
\hline 2001 & 11 & 23 & 26.83 & 26.85 & 4.85 & 4.91 & 34.59 & 34.60 & 29.47 & 29.50 \\
\hline 2001 & 11 & 24 & 26.81 & 26.83 & 4.83 & 4.85 & 34.59 & 34.60 & 29.47 & 29.49 \\
\hline 2001 & 11 & 25 & 26.80 & 26.83 & 4.84 & 4.90 & 34.60 & 34.62 & 29.45 & 29.50 \\
\hline 2001 & 11 & 26 & 26.81 & 26.83 & 4.86 & 4.88 & 34.62 & 34.62 & 29.50 & 29.51 \\
\hline 2001 & 11 & 27 & 26.77 & 26.80 & 4.84 & 4.89 & 34.62 & 34.63 & 29.47 & 29.52 \\
\hline 2001 & 11 & 28 & 26.80 & 26.81 & 4.86 & 4.89 & 34.64 & 34.64 & 29.52 & 29.54 \\
\hline 2001 & 11 & 29 & 26.74 & 26.78 & 4.82 & 4.85 & 34.63 & 34.64 & 29.47 & 29.53 \\
\hline 2001 & 11 & 30 & 26.65 & 26.72 & 4.76 & 4.80 & 34.63 & 34.65 & 29.34 & 29.43 \\
\hline 2001 & 12 & 1 & 26.66 & 26.68 & 4.77 & 4.82 & 34.66 & 34.66 & 29.30 & 29.31 \\
\hline
\end{tabular}


Table 7. Daily mean and maximum depth, in feet, of water below land surface in four monitoring wells in Oakland County, Michigan, June 2001 through March 2004 - continued $[--$, no data $]$

\begin{tabular}{|c|c|c|c|c|c|c|c|c|c|c|}
\hline \multirow[b]{2}{*}{ Year } & \multirow[b]{2}{*}{ Month } & \multirow[b]{2}{*}{ Day } & \multicolumn{2}{|c|}{$\begin{array}{c}\text { Holly State Rec. Area } \\
425116083321501\end{array}$} & \multicolumn{2}{|c|}{$\begin{array}{c}\text { Proud Lake State Rec. Area } \\
423423083324001\end{array}$} & \multicolumn{2}{|c|}{$\begin{array}{c}\text { Fish Lake Road } \\
424109083384301\end{array}$} & \multicolumn{2}{|c|}{$\begin{array}{l}\text { Teggerdine Road } \\
424133083293101\end{array}$} \\
\hline & & & Mean & Maximum & Mean & Maximum & Mean & Maximum & Mean & Maximum \\
\hline 2001 & 12 & 2 & 26.66 & 26.69 & 4.76 & 4.78 & 34.66 & 34.67 & 29.28 & 29.31 \\
\hline 2001 & 12 & 3 & 26.64 & 26.66 & 4.74 & 4.77 & 34.65 & 34.66 & 29.27 & 29.28 \\
\hline 2001 & 12 & 4 & 26.64 & 26.67 & 4.74 & 4.79 & 34.66 & 34.67 & 29.27 & 29.28 \\
\hline 2001 & 12 & 5 & 26.63 & 26.66 & 4.73 & 4.77 & 34.66 & 34.67 & 29.26 & 29.28 \\
\hline 2001 & 12 & 6 & 26.65 & 26.67 & 4.73 & 4.79 & 34.66 & 34.67 & 29.26 & 29.29 \\
\hline 2001 & 12 & 7 & 26.67 & 26.69 & 4.75 & 4.80 & 34.68 & 34.68 & 29.28 & 29.31 \\
\hline 2001 & 12 & 8 & 26.66 & 26.68 & 4.74 & 4.76 & 34.67 & 34.68 & 29.29 & 29.31 \\
\hline 2001 & 12 & 9 & 26.69 & 26.71 & 4.75 & 4.78 & 34.69 & 34.69 & 29.32 & 29.33 \\
\hline 2001 & 12 & 10 & 26.67 & 26.69 & 4.75 & 4.80 & 34.69 & 34.70 & 29.33 & 29.34 \\
\hline 2001 & 12 & 11 & 26.69 & 26.71 & 4.76 & 4.81 & 34.70 & 34.70 & 29.35 & 29.37 \\
\hline 2001 & 12 & 12 & 26.65 & 26.69 & 4.74 & 4.80 & 34.68 & 34.69 & 29.35 & 29.37 \\
\hline 2001 & 12 & 13 & 26.62 & 26.64 & 4.72 & 4.75 & 34.66 & 34.67 & 29.31 & 29.35 \\
\hline 2001 & 12 & 14 & 26.61 & 26.65 & 4.72 & 4.76 & 34.66 & 34.68 & 29.31 & 29.35 \\
\hline 2001 & 12 & 15 & 26.63 & 26.64 & 4.75 & 4.76 & 34.69 & 34.69 & 29.37 & 29.38 \\
\hline 2001 & 12 & 16 & 26.58 & 26.62 & 4.72 & 4.76 & 34.67 & 34.69 & 29.35 & 29.38 \\
\hline 2001 & 12 & 17 & 26.51 & 26.55 & 4.67 & 4.71 & 34.63 & 34.65 & 29.27 & 29.31 \\
\hline 2001 & 12 & 18 & 26.52 & 26.54 & 4.68 & 4.74 & 34.64 & 34.64 & 29.25 & 29.26 \\
\hline 2001 & 12 & 19 & 26.51 & 26.55 & 4.68 & 4.74 & 34.63 & 34.63 & 29.25 & 29.26 \\
\hline 2001 & 12 & 20 & 26.54 & 26.56 & 4.70 & 4.72 & 34.64 & 34.65 & 29.27 & 29.28 \\
\hline 2001 & 12 & 21 & 26.56 & 26.59 & 4.71 & 4.73 & 34.65 & 34.65 & 29.31 & 29.34 \\
\hline 2001 & 12 & 22 & 26.52 & 26.58 & 4.69 & 4.72 & 34.63 & 34.65 & 29.29 & 29.34 \\
\hline 2001 & 12 & 23 & 26.49 & 26.53 & 4.68 & 4.70 & 34.60 & 34.61 & 29.24 & 29.27 \\
\hline 2001 & 12 & 24 & 26.54 & 26.57 & 4.69 & 4.72 & 34.60 & 34.61 & 29.24 & 29.28 \\
\hline 2001 & 12 & 25 & 26.57 & 26.59 & 4.70 & 4.73 & 34.61 & 34.61 & 29.28 & 29.31 \\
\hline 2001 & 12 & 26 & 26.58 & 26.70 & 4.69 & 4.72 & 34.59 & 34.60 & 29.26 & 29.31 \\
\hline 2001 & 12 & 27 & 26.56 & 26.59 & 4.69 & 4.73 & 34.58 & 34.58 & 29.25 & 29.28 \\
\hline 2001 & 12 & 28 & 26.55 & 26.60 & 4.69 & 4.73 & 34.58 & 34.58 & 29.26 & 29.32 \\
\hline 2001 & 12 & 29 & 26.61 & 26.65 & 4.71 & 4.75 & 34.59 & 34.59 & 29.32 & 29.35 \\
\hline 2001 & 12 & 30 & 26.64 & 26.75 & 4.72 & 4.74 & 34.59 & 34.59 & 29.35 & 29.37 \\
\hline 2001 & 12 & 31 & 26.61 & 26.64 & 4.72 & 4.73 & 34.58 & 34.59 & 29.36 & 29.38 \\
\hline 2002 & 1 & 1 & -- & -- & 4.74 & 4.76 & 34.59 & 34.59 & 29.39 & 29.43 \\
\hline 2002 & 1 & 2 & -- & -- & 4.74 & 4.78 & 34.58 & 34.59 & 29.40 & 29.43 \\
\hline 2002 & 1 & 3 & -- & -- & 4.73 & 4.76 & 34.57 & 34.57 & 29.38 & 29.41 \\
\hline 2002 & 1 & 4 & -- & -- & 4.73 & 4.76 & 34.55 & 34.56 & 29.37 & 29.41 \\
\hline 2002 & 1 & 5 & 26.59 & 26.61 & 4.73 & 4.74 & 34.55 & 34.55 & 29.38 & 29.38 \\
\hline 2002 & 1 & 6 & -- & -- & 4.73 & 4.77 & 34.54 & 34.55 & 29.36 & 29.39 \\
\hline 2002 & 1 & 7 & -- & -- & 4.75 & 4.80 & 34.55 & 34.55 & 29.39 & 29.42 \\
\hline 2002 & 1 & 8 & -- & -- & 4.73 & 4.75 & 34.53 & 34.54 & 29.36 & 29.41 \\
\hline 2002 & 1 & 9 & 26.55 & 26.59 & 4.72 & 4.77 & 34.52 & 34.53 & 29.34 & 29.36 \\
\hline 2002 & 1 & 10 & 26.58 & 26.61 & 4.75 & 4.82 & 34.53 & 34.54 & 29.37 & 29.38 \\
\hline 2002 & 1 & 11 & 26.59 & 26.61 & 4.75 & 4.76 & 34.54 & 34.54 & 29.38 & 29.44 \\
\hline 2002 & 1 & 12 & -- & -- & 4.72 & 4.80 & 34.52 & 34.53 & 29.34 & 29.38 \\
\hline 2002 & 1 & 13 & -- & -- & 4.75 & 4.84 & 34.52 & 34.53 & 29.36 & 29.38 \\
\hline 2002 & 1 & 14 & -- & -- & 4.74 & 4.78 & 34.52 & 34.53 & 29.36 & 29.38 \\
\hline 2002 & 1 & 15 & -- & -- & 4.76 & 4.80 & 34.53 & 34.54 & 29.37 & 29.42 \\
\hline 2002 & 1 & 16 & -- & -- & 4.75 & 4.80 & 34.53 & 34.54 & 29.38 & 29.42 \\
\hline
\end{tabular}


Table 7. Daily mean and maximum depth, in feet, of water below land surface in four monitoring wells in Oakland County, Michigan, June 2001 through March 2004 - continued $[--$, no data $]$

\begin{tabular}{|c|c|c|c|c|c|c|c|c|c|c|}
\hline \multirow[b]{2}{*}{ Year } & \multirow[b]{2}{*}{ Month } & \multirow[b]{2}{*}{ Day } & \multicolumn{2}{|c|}{$\begin{array}{c}\text { Holly State Rec. Area } \\
425116083321501\end{array}$} & \multicolumn{2}{|c|}{$\begin{array}{c}\text { Proud Lake State Rec. Area } \\
423423083324001\end{array}$} & \multicolumn{2}{|c|}{$\begin{array}{c}\text { Fish Lake Road } \\
424109083384301\end{array}$} & \multicolumn{2}{|c|}{$\begin{array}{l}\text { Teggerdine Road } \\
424133083293101\end{array}$} \\
\hline & & & Mean & Maximum & Mean & Maximum & Mean & Maximum & Mean & Maximum \\
\hline 2002 & 1 & 17 & -- & -- & 4.74 & 4.78 & 34.52 & 34.53 & 29.36 & 29.38 \\
\hline 2002 & 1 & 18 & -- & -- & 4.76 & 4.77 & 34.53 & 34.53 & 29.38 & 29.40 \\
\hline 2002 & 1 & 19 & -- & -- & 4.74 & 4.76 & 34.51 & 34.53 & 29.35 & 29.40 \\
\hline 2002 & 1 & 20 & -- & -- & 4.75 & 4.79 & 34.51 & 34.52 & 29.36 & 29.38 \\
\hline 2002 & 1 & 21 & -- & -- & 4.75 & 4.79 & 34.51 & 34.53 & 29.34 & 29.40 \\
\hline 2002 & 1 & 22 & -- & -- & 4.77 & 4.82 & 34.53 & 34.53 & 29.39 & 29.41 \\
\hline 2002 & 1 & 23 & -- & -- & 4.74 & 4.79 & 34.51 & 34.52 & 29.35 & 29.35 \\
\hline 2002 & 1 & 24 & -- & -- & 4.75 & 4.78 & 34.52 & 34.52 & 29.34 & 29.36 \\
\hline 2002 & 1 & 25 & -- & -- & 4.75 & 4.79 & 34.52 & 34.53 & 29.35 & 29.36 \\
\hline 2002 & 1 & 26 & -- & -- & 4.76 & 4.79 & 34.53 & 34.53 & 29.34 & 29.36 \\
\hline 2002 & 1 & 27 & -- & -- & 4.75 & 4.80 & 34.52 & 34.53 & 29.34 & 29.36 \\
\hline 2002 & 1 & 28 & -- & -- & 4.74 & 4.80 & 34.52 & 34.52 & 29.32 & 29.35 \\
\hline 2002 & 1 & 29 & -- & -- & 4.75 & 4.82 & 34.53 & 34.53 & 29.34 & 29.35 \\
\hline 2002 & 1 & 30 & -- & -- & 4.77 & 4.81 & 34.54 & 34.55 & 29.38 & 29.41 \\
\hline 2002 & 1 & 31 & -- & -- & 4.73 & 4.78 & 34.53 & 34.55 & 29.34 & 29.40 \\
\hline 2002 & 2 & 1 & -- & -- & 4.73 & 4.83 & 34.53 & 34.56 & 29.32 & 29.38 \\
\hline 2002 & 2 & 2 & -- & -- & 4.76 & 4.83 & 34.55 & 34.56 & 29.37 & 29.39 \\
\hline 2002 & 2 & 3 & -- & -- & 4.72 & 4.78 & 34.53 & 34.54 & 29.31 & 29.35 \\
\hline 2002 & 2 & 4 & -- & -- & 4.76 & 4.83 & 34.55 & 34.56 & 29.36 & 29.41 \\
\hline 2002 & 2 & 5 & -- & -- & 4.76 & 4.83 & 34.55 & 34.56 & 29.36 & 29.39 \\
\hline 2002 & 2 & 6 & -- & -- & 4.74 & 4.81 & 34.54 & 34.55 & 29.34 & 29.37 \\
\hline 2002 & 2 & 7 & -- & -- & 4.73 & 4.78 & 34.54 & 34.55 & 29.33 & 29.35 \\
\hline 2002 & 2 & 8 & -- & -- & 4.75 & 4.81 & 34.55 & 34.56 & 29.35 & 29.40 \\
\hline 2002 & 2 & 9 & -- & -- & 4.76 & 4.79 & 34.56 & 34.57 & 29.36 & 29.40 \\
\hline 2002 & 2 & 10 & -- & -- & 4.73 & 4.85 & 34.55 & 34.57 & 29.33 & 29.35 \\
\hline 2002 & 2 & 11 & -- & -- & 4.82 & 5.03 & 34.56 & 34.58 & 29.33 & 29.36 \\
\hline 2002 & 2 & 12 & -- & -- & 4.70 & 4.77 & 34.55 & 34.57 & 29.28 & 29.33 \\
\hline 2002 & 2 & 13 & -- & -- & 4.75 & 4.78 & 34.58 & 34.58 & 29.33 & 29.34 \\
\hline 2002 & 2 & 14 & -- & -- & 4.72 & 4.77 & 34.57 & 34.57 & 29.32 & 29.34 \\
\hline 2002 & 2 & 15 & -- & -- & 4.69 & 4.74 & 34.55 & 34.56 & 29.28 & 29.30 \\
\hline 2002 & 2 & 16 & -- & -- & 4.70 & 4.80 & 34.55 & 34.57 & 29.27 & 29.29 \\
\hline 2002 & 2 & 17 & -- & -- & 4.73 & 4.81 & 34.58 & 34.59 & 29.30 & 29.33 \\
\hline 2002 & 2 & 18 & -- & -- & 4.72 & 4.78 & 34.59 & 34.59 & 29.31 & 29.33 \\
\hline 2002 & 2 & 19 & -- & -- & 4.69 & 4.72 & 34.57 & 34.58 & 29.28 & 29.30 \\
\hline 2002 & 2 & 20 & -- & -- & 4.64 & 4.68 & 34.55 & 34.56 & 29.22 & 29.26 \\
\hline 2002 & 2 & 21 & 26.21 & 26.23 & 4.63 & 4.68 & 34.57 & 34.59 & 29.18 & 29.19 \\
\hline 2002 & 2 & 22 & 26.21 & 26.21 & 4.62 & 4.66 & 34.59 & 34.59 & 29.17 & 29.18 \\
\hline 2002 & 2 & 23 & 26.18 & 26.20 & 4.59 & 4.63 & 34.58 & 34.59 & 29.14 & 29.17 \\
\hline 2002 & 2 & 24 & 26.16 & 26.19 & 4.57 & 4.63 & 34.56 & 34.57 & 29.12 & 29.14 \\
\hline 2002 & 2 & 25 & 26.14 & 26.17 & 4.55 & 4.62 & 34.54 & 34.55 & 29.10 & 29.11 \\
\hline 2002 & 2 & 26 & 26.11 & 26.14 & 4.53 & 4.59 & 34.52 & 34.53 & 29.08 & 29.09 \\
\hline 2002 & 2 & 27 & 26.14 & 26.18 & 4.55 & 4.60 & 34.53 & 34.53 & 29.09 & 29.12 \\
\hline 2002 & 2 & 28 & 26.16 & 26.20 & 4.55 & 4.59 & 34.53 & 34.53 & 29.10 & 29.12 \\
\hline 2002 & 3 & 1 & 26.17 & 26.19 & 4.56 & 4.61 & 34.54 & 34.54 & 29.12 & 29.14 \\
\hline 2002 & 3 & 2 & 26.07 & 26.15 & 4.49 & 4.55 & 34.51 & 34.53 & 29.08 & 29.13 \\
\hline 2002 & 3 & 3 & 26.04 & 26.11 & 4.47 & 4.53 & 34.50 & 34.52 & 29.03 & 29.05 \\
\hline
\end{tabular}


Table 7. Daily mean and maximum depth, in feet, of water below land surface in four monitoring wells in Oakland County, Michigan, June 2001 through March 2004 - continued $[--$, no data $]$

\begin{tabular}{|c|c|c|c|c|c|c|c|c|c|c|}
\hline \multirow[b]{2}{*}{ Year } & \multirow[b]{2}{*}{ Month } & \multirow[b]{2}{*}{ Day } & \multicolumn{2}{|c|}{$\begin{array}{c}\text { Holly State Rec. Area } \\
425116083321501\end{array}$} & \multicolumn{2}{|c|}{$\begin{array}{c}\text { Proud Lake State Rec. Area } \\
423423083324001\end{array}$} & \multicolumn{2}{|c|}{$\begin{array}{l}\text { Fish Lake Road } \\
424109083384301\end{array}$} & \multicolumn{2}{|c|}{$\begin{array}{l}\text { Teggerdine Road } \\
424133083293101\end{array}$} \\
\hline & & & Mean & Maximum & Mean & Maximum & Mean & Maximum & Mean & Maximum \\
\hline 2002 & 3 & 4 & 26.08 & 26.15 & 4.48 & 4.51 & 34.51 & 34.52 & 29.04 & 29.04 \\
\hline 2002 & 3 & 5 & 26.05 & 26.09 & 4.46 & 4.51 & 34.50 & 34.51 & 29.03 & 29.05 \\
\hline 2002 & 3 & 6 & 26.02 & 26.05 & 4.44 & 4.47 & 34.49 & 34.49 & 29.03 & 29.03 \\
\hline 2002 & 3 & 7 & 26.03 & 26.05 & 4.44 & 4.49 & 34.48 & 34.49 & 29.04 & 29.04 \\
\hline 2002 & 3 & 8 & 25.97 & 26.01 & 4.41 & 4.44 & 34.47 & 34.47 & 29.00 & 29.02 \\
\hline 2002 & 3 & 9 & 25.86 & 25.94 & 4.36 & 4.40 & 34.45 & 34.47 & 28.95 & 28.98 \\
\hline 2002 & 3 & 10 & 25.91 & 25.95 & 4.39 & 4.42 & 34.47 & 34.47 & 28.94 & 28.97 \\
\hline 2002 & 3 & 11 & 25.84 & 25.95 & 4.36 & 4.42 & 34.44 & 34.46 & 28.91 & 28.96 \\
\hline 2002 & 3 & 12 & 25.80 & 25.83 & 4.34 & 4.37 & 34.42 & 34.43 & 28.88 & 28.90 \\
\hline 2002 & 3 & 13 & 25.78 & 25.83 & 4.32 & 4.36 & 34.40 & 34.41 & 28.86 & 28.88 \\
\hline 2002 & 3 & 14 & 25.81 & 25.87 & 4.32 & 4.35 & 34.38 & 34.39 & 28.87 & 28.88 \\
\hline 2002 & 3 & 15 & 25.79 & 25.87 & 4.30 & 4.33 & 34.37 & 34.37 & 28.85 & 28.90 \\
\hline 2002 & 3 & 16 & 25.89 & 25.93 & 4.33 & 4.38 & 34.36 & 34.37 & 28.91 & 28.93 \\
\hline 2002 & 3 & 17 & 25.83 & 25.98 & 4.29 & 4.34 & 34.34 & 34.36 & 28.91 & 28.93 \\
\hline 2002 & 3 & 18 & 25.82 & 25.84 & 4.30 & 4.35 & 34.33 & 34.34 & 28.91 & 28.93 \\
\hline 2002 & 3 & 19 & 25.81 & 25.87 & 4.29 & 4.33 & 34.32 & 34.33 & 28.92 & 28.93 \\
\hline 2002 & 3 & 20 & -- & 25.82 & 4.27 & 4.30 & 34.30 & 34.30 & 28.90 & 28.94 \\
\hline 2002 & 3 & 21 & -- & -- & 4.28 & 4.32 & 34.28 & 34.29 & 28.92 & 28.94 \\
\hline 2002 & 3 & 22 & -- & -- & 4.27 & 4.31 & 34.27 & 34.28 & 28.93 & 28.95 \\
\hline 2002 & 3 & 23 & -- & -- & 4.26 & 4.29 & 34.25 & 34.26 & 28.91 & 28.94 \\
\hline 2002 & 3 & 24 & -- & -- & 4.28 & 4.33 & 34.24 & 34.25 & 28.95 & 28.97 \\
\hline 2002 & 3 & 25 & -- & -- & 4.30 & 4.33 & 34.23 & 34.24 & 28.97 & 28.98 \\
\hline 2002 & 3 & 26 & -- & -- & 4.27 & 4.34 & 34.21 & 34.22 & 28.96 & 28.98 \\
\hline 2002 & 3 & 27 & -- & -- & 4.29 & 4.34 & 34.20 & 34.21 & 28.98 & 28.99 \\
\hline 2002 & 3 & 28 & -- & -- & 4.27 & 4.35 & 34.18 & 34.19 & 28.97 & 28.99 \\
\hline 2002 & 3 & 29 & -- & -- & 4.25 & 4.29 & 34.16 & 34.17 & 28.94 & 28.95 \\
\hline 2002 & 3 & 30 & -- & -- & 4.27 & 4.30 & 34.16 & 34.16 & 28.96 & 28.99 \\
\hline 2002 & 3 & 31 & -- & -- & 4.28 & 4.32 & 34.15 & 34.15 & 28.97 & 29.00 \\
\hline 2002 & 4 & 1 & -- & -- & 4.29 & 4.34 & 34.14 & 34.14 & 28.98 & 29.01 \\
\hline 2002 & 4 & 2 & -- & -- & 4.25 & 4.30 & 34.12 & 34.13 & 28.94 & 28.98 \\
\hline 2002 & 4 & 3 & -- & -- & 4.28 & 4.33 & 34.14 & 34.15 & 28.94 & 28.97 \\
\hline 2002 & 4 & 4 & -- & -- & 4.29 & 4.33 & 34.14 & 34.14 & 28.96 & 28.97 \\
\hline 2002 & 4 & 5 & -- & -- & 4.27 & 4.31 & 34.12 & 34.13 & 28.94 & 28.95 \\
\hline 2002 & 4 & 6 & -- & -- & 4.27 & 4.30 & 34.12 & 34.12 & 28.95 & 28.96 \\
\hline 2002 & 4 & 7 & -- & -- & 4.25 & 4.31 & 34.11 & 34.11 & 28.93 & 28.95 \\
\hline 2002 & 4 & 8 & -- & -- & 4.23 & 4.29 & 34.10 & 34.11 & 28.89 & 28.92 \\
\hline 2002 & 4 & 9 & -- & -- & 4.23 & 4.29 & 34.11 & 34.13 & 28.85 & 28.87 \\
\hline 2002 & 4 & 10 & -- & -- & 4.22 & 4.28 & 34.12 & 34.13 & 28.85 & 28.87 \\
\hline 2002 & 4 & 11 & -- & -- & 4.20 & 4.22 & 34.10 & 34.11 & 28.81 & 28.82 \\
\hline 2002 & 4 & 12 & -- & -- & 4.18 & 4.20 & 34.09 & 34.09 & 28.79 & 28.80 \\
\hline 2002 & 4 & 13 & -- & -- & 4.16 & 4.20 & 34.08 & 34.09 & 28.78 & 28.78 \\
\hline 2002 & 4 & 14 & -- & -- & 4.14 & 4.19 & 34.07 & 34.08 & 28.76 & 28.78 \\
\hline 2002 & 4 & 15 & -- & -- & 4.15 & 4.20 & 34.06 & 34.07 & 28.75 & 28.76 \\
\hline 2002 & 4 & 16 & -- & -- & 4.16 & 4.20 & 34.07 & 34.07 & 28.77 & 28.79 \\
\hline 2002 & 4 & 17 & -- & -- & 4.16 & 4.20 & 34.06 & 34.07 & 28.79 & 28.80 \\
\hline 2002 & 4 & 18 & -- & -- & 4.15 & 4.18 & 34.06 & 34.07 & 28.79 & 28.81 \\
\hline
\end{tabular}


Table 7. Daily mean and maximum depth, in feet, of water below land surface in four monitoring wells in Oakland County, Michigan, June 2001 through March 2004 - continued $[--$, no data $]$

\begin{tabular}{|c|c|c|c|c|c|c|c|c|c|c|}
\hline \multirow[b]{2}{*}{ Year } & \multirow[b]{2}{*}{ Month } & \multirow[b]{2}{*}{ Day } & \multicolumn{2}{|c|}{$\begin{array}{c}\text { Holly State Rec. Area } \\
425116083321501\end{array}$} & \multicolumn{2}{|c|}{$\begin{array}{c}\text { Proud Lake State Rec. Area } \\
423423083324001\end{array}$} & \multicolumn{2}{|c|}{$\begin{array}{c}\text { Fish Lake Road } \\
424109083384301\end{array}$} & \multicolumn{2}{|c|}{$\begin{array}{l}\text { Teggerdine Road } \\
424133083293101\end{array}$} \\
\hline & & & Mean & Maximum & Mean & Maximum & Mean & Maximum & Mean & Maximum \\
\hline 2002 & 4 & 19 & -- & -- & 4.15 & 4.20 & 34.06 & 34.07 & 28.80 & 28.83 \\
\hline 2002 & 4 & 20 & -- & -- & 4.16 & 4.20 & 34.06 & 34.08 & 28.83 & 28.84 \\
\hline 2002 & 4 & 21 & -- & -- & 4.15 & 4.17 & 34.05 & 34.06 & 28.83 & 28.85 \\
\hline 2002 & 4 & 22 & -- & -- & 4.16 & 4.21 & 34.05 & 34.06 & 28.83 & 28.86 \\
\hline 2002 & 4 & 23 & -- & -- & 4.18 & 4.20 & 34.06 & 34.07 & 28.85 & 28.87 \\
\hline 2002 & 4 & 24 & -- & -- & 4.16 & 4.20 & 34.05 & 34.06 & 28.83 & 28.87 \\
\hline 2002 & 4 & 25 & -- & -- & 4.18 & 4.24 & 34.06 & 34.06 & 28.85 & 28.88 \\
\hline 2002 & 4 & 26 & 25.96 & 25.98 & 4.21 & 4.24 & 34.07 & 34.08 & 28.88 & 28.93 \\
\hline 2002 & 4 & 27 & 25.94 & 25.99 & 4.19 & 4.23 & 34.06 & 34.08 & 28.89 & 28.94 \\
\hline 2002 & 4 & 28 & 25.85 & 25.92 & 4.14 & 4.19 & 34.04 & 34.07 & 28.81 & 28.85 \\
\hline 2002 & 4 & 29 & 25.90 & 25.92 & 4.19 & 4.22 & 34.07 & 34.07 & 28.85 & 28.87 \\
\hline 2002 & 4 & 30 & 25.89 & 25.91 & 4.18 & 4.22 & 34.06 & 34.07 & 28.85 & 28.87 \\
\hline 2002 & 5 & 1 & 25.89 & 25.91 & 4.19 & 4.22 & 34.06 & 34.07 & 28.87 & 28.89 \\
\hline 2002 & 5 & 2 & 25.83 & 25.86 & 4.17 & 4.21 & 34.06 & 34.08 & 28.83 & 28.87 \\
\hline 2002 & 5 & 3 & 25.89 & 25.92 & 4.22 & 4.27 & 34.09 & 34.10 & 28.88 & 28.90 \\
\hline 2002 & 5 & 4 & 25.88 & 25.90 & 4.23 & 4.26 & 34.09 & 34.10 & 28.89 & 28.94 \\
\hline 2002 & 5 & 5 & 25.88 & 25.90 & 4.24 & 4.26 & 34.08 & 34.09 & 28.90 & 28.91 \\
\hline 2002 & 5 & 6 & 25.85 & 25.88 & 4.22 & 4.29 & 34.06 & 34.07 & 28.88 & 28.90 \\
\hline 2002 & 5 & 7 & 25.87 & 25.90 & 4.24 & 4.38 & 34.06 & 34.07 & 28.88 & 28.92 \\
\hline 2002 & 5 & 8 & 25.87 & 25.90 & 4.23 & 4.27 & 34.06 & 34.07 & 28.89 & 28.92 \\
\hline 2002 & 5 & 9 & 25.83 & 25.88 & 4.21 & 4.25 & 34.04 & 34.06 & 28.86 & 28.91 \\
\hline 2002 & 5 & 10 & 25.90 & 25.94 & 4.27 & 4.30 & 34.08 & 34.09 & 28.92 & 28.94 \\
\hline 2002 & 5 & 11 & 25.90 & 25.93 & 4.28 & 4.31 & 34.08 & 34.09 & 28.94 & 28.98 \\
\hline 2002 & 5 & 12 & 25.78 & 25.85 & 4.20 & 4.26 & 34.04 & 34.06 & 28.85 & 28.91 \\
\hline 2002 & 5 & 13 & 25.76 & 25.77 & 4.20 & 4.24 & 34.04 & 34.04 & 28.80 & 28.84 \\
\hline 2002 & 5 & 14 & 25.77 & 25.81 & 4.21 & 4.26 & 34.03 & 34.05 & 28.79 & 28.82 \\
\hline 2002 & 5 & 15 & 25.80 & 25.82 & 4.24 & 4.29 & 34.04 & 34.05 & 28.81 & 28.85 \\
\hline 2002 & 5 & 16 & 25.74 & 25.77 & 4.22 & 4.27 & 34.03 & 34.04 & 28.79 & 28.81 \\
\hline 2002 & 5 & 17 & 25.68 & 25.70 & 4.23 & 4.26 & 34.03 & 34.04 & 28.80 & 28.82 \\
\hline 2002 & 5 & 18 & 25.69 & 25.71 & 4.24 & 4.27 & 34.03 & 34.03 & 28.81 & 28.85 \\
\hline 2002 & 5 & 19 & 25.71 & 25.72 & 4.26 & 4.30 & 34.02 & 34.03 & 28.84 & 28.87 \\
\hline 2002 & 5 & 20 & 25.72 & 25.73 & 4.27 & 4.32 & 34.02 & 34.02 & 28.85 & 28.90 \\
\hline 2002 & 5 & 21 & 25.72 & 25.74 & 4.27 & 4.30 & 34.01 & 34.02 & 28.86 & 28.90 \\
\hline 2002 & 5 & 22 & 25.71 & 25.74 & 4.28 & 4.33 & 34.01 & 34.01 & 28.87 & 28.91 \\
\hline 2002 & 5 & 23 & 25.70 & 25.73 & 4.28 & 4.32 & 34.00 & 34.00 & 28.83 & 28.87 \\
\hline 2002 & 5 & 24 & 25.76 & 25.80 & 4.31 & 4.34 & 34.00 & 34.01 & 28.87 & 28.91 \\
\hline 2002 & 5 & 25 & 25.76 & 25.79 & 4.31 & 4.33 & 33.99 & 34.00 & 28.87 & 28.90 \\
\hline 2002 & 5 & 26 & 25.79 & 25.81 & 4.35 & 4.37 & 33.99 & 34.00 & 28.90 & 28.93 \\
\hline 2002 & 5 & 27 & 25.80 & 25.83 & 4.37 & 4.41 & 33.98 & 33.98 & 28.91 & 28.94 \\
\hline 2002 & 5 & 28 & 25.80 & 25.82 & 4.40 & 4.44 & 33.97 & 33.98 & 28.93 & 28.98 \\
\hline 2002 & 5 & 29 & 25.80 & 25.82 & 4.40 & 4.43 & 33.96 & 33.96 & 28.94 & 28.96 \\
\hline 2002 & 5 & 30 & 25.82 & 25.84 & 4.41 & 4.47 & 33.96 & 33.96 & 28.94 & 28.96 \\
\hline 2002 & 5 & 31 & 25.84 & 25.89 & 4.39 & 4.44 & 33.95 & 33.96 & 28.94 & 28.98 \\
\hline 2002 & 6 & 1 & 25.90 & 25.94 & 4.44 & 4.48 & 33.95 & 33.96 & 28.98 & 29.01 \\
\hline 2002 & 6 & 2 & 25.97 & 26.01 & 4.51 & 4.55 & 33.96 & 33.97 & 29.02 & 29.07 \\
\hline 2002 & 6 & 3 & 25.97 & 26.00 & 4.51 & 4.56 & 33.96 & 33.97 & 29.03 & 29.10 \\
\hline
\end{tabular}


Table 7. Daily mean and maximum depth, in feet, of water below land surface in four monitoring wells in Oakland County, Michigan, June 2001 through March 2004 - continued $[--$, no data $]$

\begin{tabular}{|c|c|c|c|c|c|c|c|c|c|c|}
\hline \multirow[b]{2}{*}{ Year } & \multirow[b]{2}{*}{ Month } & \multirow[b]{2}{*}{ Day } & \multicolumn{2}{|c|}{$\begin{array}{c}\text { Holly State Rec. Area } \\
425116083321501\end{array}$} & \multicolumn{2}{|c|}{$\begin{array}{l}\text { Proud Lake State Rec. Area } \\
423423083324001\end{array}$} & \multicolumn{2}{|c|}{$\begin{array}{l}\text { Fish Lake Road } \\
424109083384301\end{array}$} & \multicolumn{2}{|c|}{$\begin{array}{l}\text { Teggerdine Road } \\
424133083293101\end{array}$} \\
\hline & & & Mean & Maximum & Mean & Maximum & Mean & Maximum & Mean & Maximum \\
\hline 2002 & 6 & 4 & 25.89 & 25.93 & 4.48 & 4.76 & 33.95 & 33.96 & 28.97 & 29.02 \\
\hline 2002 & 6 & 5 & 25.89 & 25.92 & 4.47 & 4.51 & 33.95 & 33.95 & 28.94 & 28.98 \\
\hline 2002 & 6 & 6 & 25.92 & 25.94 & 4.49 & 4.55 & 33.95 & 33.95 & 28.97 & 29.01 \\
\hline 2002 & 6 & 7 & 25.95 & 25.97 & 4.51 & 4.54 & 33.94 & 33.95 & 28.97 & 29.05 \\
\hline 2002 & 6 & 8 & 25.99 & 26.02 & 4.53 & 4.56 & 33.94 & 33.94 & 28.99 & 29.05 \\
\hline 2002 & 6 & 9 & 26.03 & 26.05 & 4.57 & 4.61 & 33.93 & 33.93 & 29.02 & 29.09 \\
\hline 2002 & 6 & 10 & 26.05 & 26.07 & 4.67 & 5.12 & 33.92 & 33.93 & 29.03 & 29.07 \\
\hline 2002 & 6 & 11 & 26.07 & 26.09 & 4.99 & 5.16 & 33.91 & 33.92 & 29.04 & 29.09 \\
\hline 2002 & 6 & 12 & 26.12 & 26.16 & 4.64 & 4.68 & 33.92 & 33.93 & 29.06 & 29.14 \\
\hline 2002 & 6 & 13 & 26.15 & 26.16 & 4.66 & 4.73 & 33.92 & 33.93 & 29.08 & 29.14 \\
\hline 2002 & 6 & 14 & 26.15 & 26.17 & 4.63 & 4.67 & 33.92 & 33.92 & 29.07 & 29.13 \\
\hline 2002 & 6 & 15 & 26.17 & 26.18 & 4.64 & 4.67 & 33.93 & 33.94 & 29.07 & 29.14 \\
\hline 2002 & 6 & 16 & 26.19 & 26.22 & 4.66 & 4.81 & 33.95 & 33.95 & 29.08 & 29.14 \\
\hline 2002 & 6 & 17 & 26.22 & 26.24 & 4.68 & 4.71 & 33.96 & 33.97 & 29.10 & 29.17 \\
\hline 2002 & 6 & 18 & 26.25 & 26.29 & 4.72 & 4.84 & 33.98 & 33.99 & 29.15 & 29.25 \\
\hline 2002 & 6 & 19 & 26.28 & 26.32 & 4.77 & 4.81 & 34.00 & 34.00 & 29.15 & 29.27 \\
\hline 2002 & 6 & 20 & 26.32 & 26.34 & 4.82 & 4.86 & 34.01 & 34.01 & 29.19 & 29.27 \\
\hline 2002 & 6 & 21 & 26.35 & 26.36 & 4.84 & 4.89 & 34.01 & 34.01 & 29.21 & 29.25 \\
\hline 2002 & 6 & 22 & 26.36 & 26.38 & 4.85 & 4.90 & 34.00 & 34.01 & 29.22 & 29.29 \\
\hline 2002 & 6 & 23 & 26.39 & 26.43 & 4.90 & 4.95 & 33.99 & 33.99 & 29.25 & 29.32 \\
\hline 2002 & 6 & 24 & 26.43 & 26.46 & 4.90 & 4.94 & 33.99 & 33.99 & 29.28 & 29.33 \\
\hline 2002 & 6 & 25 & 26.47 & 26.49 & 4.93 & 4.96 & 33.99 & 33.99 & 29.31 & 29.41 \\
\hline 2002 & 6 & 26 & 26.49 & 26.52 & 4.92 & 4.95 & 33.99 & 33.99 & 29.31 & 29.36 \\
\hline 2002 & 6 & 27 & 26.53 & 26.58 & 4.94 & 4.99 & 34.00 & 34.02 & 29.32 & 29.46 \\
\hline 2002 & 6 & 28 & 26.59 & 26.63 & 5.00 & 5.04 & 34.03 & 34.04 & 29.36 & 29.45 \\
\hline 2002 & 6 & 29 & 26.64 & 26.67 & 5.04 & 5.09 & 34.05 & 34.06 & 29.39 & 29.46 \\
\hline 2002 & 6 & 30 & 26.68 & 26.72 & 5.08 & 5.15 & 34.06 & 34.06 & 29.42 & 29.57 \\
\hline 2002 & 7 & 1 & 26.71 & 26.74 & 5.12 & 5.18 & 34.07 & 34.07 & -- & -- \\
\hline 2002 & 7 & 2 & 26.76 & 26.79 & 5.15 & 5.19 & 34.08 & 34.08 & -- & -- \\
\hline 2002 & 7 & 3 & 26.80 & 26.84 & 5.20 & 5.40 & 34.08 & 34.09 & -- & -- \\
\hline 2002 & 7 & 4 & 26.86 & 26.90 & 5.25 & 5.33 & 34.10 & 34.11 & -- & -- \\
\hline 2002 & 7 & 5 & 26.91 & 26.92 & 5.29 & 5.33 & 34.12 & 34.13 & -- & -- \\
\hline 2002 & 7 & 6 & 26.93 & 26.95 & 5.31 & 5.34 & 34.13 & 34.14 & -- & -- \\
\hline 2002 & 7 & 7 & 26.95 & 26.99 & 5.34 & 5.44 & 34.14 & 34.14 & -- & -- \\
\hline 2002 & 7 & 8 & 26.97 & 27.02 & 5.37 & 5.46 & 34.14 & 34.15 & -- & -- \\
\hline 2002 & 7 & 9 & 27.01 & 27.06 & 5.34 & 5.39 & 34.15 & 34.16 & -- & -- \\
\hline 2002 & 7 & 10 & 27.05 & 27.11 & 5.35 & 5.41 & 34.17 & 34.18 & -- & -- \\
\hline 2002 & 7 & 11 & 27.08 & 27.10 & 5.41 & 5.46 & 34.19 & 34.19 & -- & -- \\
\hline 2002 & 7 & 12 & 27.09 & 27.11 & 5.45 & 5.52 & 34.19 & 34.20 & -- & -- \\
\hline 2002 & 7 & 13 & 27.13 & 27.16 & 5.49 & 5.55 & 34.20 & 34.21 & -- & -- \\
\hline 2002 & 7 & 14 & 27.18 & 27.24 & 5.53 & 5.61 & 34.22 & 34.22 & -- & -- \\
\hline 2002 & 7 & 15 & 27.22 & 27.27 & 5.57 & 5.60 & 34.23 & 34.24 & -- & -- \\
\hline 2002 & 7 & 16 & 27.27 & 27.33 & 5.60 & 5.64 & 34.25 & 34.25 & -- & -- \\
\hline 2002 & 7 & 17 & 27.30 & 27.35 & 5.64 & 5.69 & 34.26 & 34.26 & -- & -- \\
\hline 2002 & 7 & 18 & 27.33 & 27.38 & 5.66 & 5.71 & 34.27 & 34.27 & -- & -- \\
\hline 2002 & 7 & 19 & 27.36 & 27.41 & 5.67 & 5.71 & 34.28 & 34.29 & -- & -- \\
\hline
\end{tabular}


Table 7. Daily mean and maximum depth, in feet, of water below land surface in four monitoring wells in Oakland County, Michigan, June 2001 through March 2004 - continued $[--$, no data $]$

\begin{tabular}{|c|c|c|c|c|c|c|c|c|c|c|}
\hline \multirow[b]{2}{*}{ Year } & \multirow[b]{2}{*}{ Month } & \multirow[b]{2}{*}{ Day } & \multicolumn{2}{|c|}{$\begin{array}{c}\text { Holly State Rec. Area } \\
425116083321501\end{array}$} & \multicolumn{2}{|c|}{$\begin{array}{l}\text { Proud Lake State Rec. Area } \\
423423083324001\end{array}$} & \multicolumn{2}{|c|}{$\begin{array}{l}\text { Fish Lake Road } \\
424109083384301\end{array}$} & \multicolumn{2}{|c|}{$\begin{array}{l}\text { Teggerdine Road } \\
424133083293101\end{array}$} \\
\hline & & & Mean & Maximum & Mean & Maximum & Mean & Maximum & Mean & Maximum \\
\hline 2002 & 7 & 20 & 27.39 & 27.42 & 5.72 & 5.77 & 34.30 & 34.30 & -- & -- \\
\hline 2002 & 7 & 21 & 27.38 & 27.42 & 5.71 & 5.77 & 34.31 & 34.31 & -- & -- \\
\hline 2002 & 7 & 22 & 27.32 & 27.35 & 5.64 & 5.68 & 34.32 & 34.33 & -- & -- \\
\hline 2002 & 7 & 23 & 27.29 & 27.30 & 5.66 & 5.71 & 34.33 & 34.33 & -- & -- \\
\hline 2002 & 7 & 24 & 27.26 & 27.29 & 5.70 & 5.77 & 34.33 & 34.34 & -- & -- \\
\hline 2002 & 7 & 25 & 27.26 & 27.28 & 5.72 & 5.78 & 34.34 & 34.34 & -- & -- \\
\hline 2002 & 7 & 26 & 27.23 & 27.26 & 5.67 & 5.74 & 34.34 & 34.34 & -- & -- \\
\hline 2002 & 7 & 27 & 27.20 & 27.22 & 5.64 & 5.67 & 34.34 & 34.35 & -- & -- \\
\hline 2002 & 7 & 28 & 27.16 & 27.18 & 5.61 & 5.64 & 34.33 & 34.33 & -- & -- \\
\hline 2002 & 7 & 29 & 27.15 & 27.17 & -- & 5.65 & 34.32 & 34.33 & -- & -- \\
\hline 2002 & 7 & 30 & 27.11 & 27.12 & -- & -- & 34.32 & 34.32 & -- & -- \\
\hline 2002 & 7 & 31 & 27.12 & 27.13 & -- & -- & 34.32 & 34.32 & -- & -- \\
\hline 2002 & 8 & 1 & 27.14 & 27.17 & -- & -- & 34.32 & 34.32 & -- & -- \\
\hline 2002 & 8 & 2 & 27.19 & 27.23 & -- & -- & 34.33 & 34.34 & -- & -- \\
\hline 2002 & 8 & 3 & 27.23 & 27.26 & -- & -- & 34.34 & 34.34 & -- & -- \\
\hline 2002 & 8 & 4 & 27.25 & 27.27 & -- & -- & 34.34 & 34.34 & -- & -- \\
\hline 2002 & 8 & 5 & 27.28 & 27.31 & -- & -- & 34.34 & 34.35 & -- & -- \\
\hline 2002 & 8 & 6 & 27.32 & 27.37 & -- & -- & 34.35 & 34.36 & -- & -- \\
\hline 2002 & 8 & 7 & 27.33 & 27.35 & -- & -- & 34.36 & 34.36 & -- & -- \\
\hline 2002 & 8 & 8 & 27.36 & 27.38 & -- & -- & 34.37 & 34.37 & -- & -- \\
\hline 2002 & 8 & 9 & 27.38 & 27.41 & -- & -- & 34.37 & 34.38 & -- & -- \\
\hline 2002 & 8 & 10 & 27.40 & 27.43 & -- & -- & 34.38 & 34.38 & -- & -- \\
\hline 2002 & 8 & 11 & 27.45 & 27.50 & -- & -- & 34.38 & 34.39 & -- & -- \\
\hline 2002 & 8 & 12 & 27.47 & 27.52 & -- & -- & 34.39 & 34.39 & -- & -- \\
\hline 2002 & 8 & 13 & 27.50 & 27.53 & -- & -- & 34.39 & 34.40 & -- & -- \\
\hline 2002 & 8 & 14 & 27.46 & 27.50 & -- & -- & 34.40 & 34.41 & -- & -- \\
\hline 2002 & 8 & 15 & 27.40 & 27.42 & -- & -- & 34.41 & 34.42 & -- & -- \\
\hline 2002 & 8 & 16 & 27.39 & 27.41 & -- & -- & 34.42 & 34.43 & -- & -- \\
\hline 2002 & 8 & 17 & 27.37 & 27.39 & -- & -- & 34.43 & 34.43 & -- & -- \\
\hline 2002 & 8 & 18 & 27.40 & 27.44 & -- & -- & 34.44 & 34.44 & -- & -- \\
\hline 2002 & 8 & 19 & 27.39 & 27.41 & -- & -- & 34.44 & 34.45 & -- & -- \\
\hline 2002 & 8 & 20 & 27.39 & 27.41 & -- & -- & 34.46 & 34.47 & -- & -- \\
\hline 2002 & 8 & 21 & 27.39 & 27.41 & -- & -- & 34.47 & 34.47 & -- & -- \\
\hline 2002 & 8 & 22 & 27.39 & 27.41 & -- & -- & 34.48 & 34.48 & -- & -- \\
\hline 2002 & 8 & 23 & 27.38 & 27.41 & -- & -- & 34.48 & 34.49 & -- & -- \\
\hline 2002 & 8 & 24 & 27.37 & 27.40 & -- & -- & 34.49 & 34.50 & -- & -- \\
\hline 2002 & 8 & 25 & 27.39 & 27.41 & -- & -- & 34.50 & 34.51 & -- & -- \\
\hline 2002 & 8 & 26 & 27.41 & 27.45 & -- & -- & 34.51 & 34.51 & -- & -- \\
\hline 2002 & 8 & 27 & 27.45 & 27.48 & -- & -- & 34.52 & 34.53 & -- & -- \\
\hline 2002 & 8 & 28 & 27.46 & 27.50 & -- & -- & 34.53 & 34.54 & -- & -- \\
\hline 2002 & 8 & 29 & 27.48 & 27.54 & -- & -- & 34.54 & 34.55 & -- & -- \\
\hline 2002 & 8 & 30 & 27.51 & 27.53 & 6.12 & 6.15 & 34.55 & 34.56 & -- & -- \\
\hline 2002 & 8 & 31 & 27.54 & 27.55 & 6.15 & 6.18 & 34.56 & 34.57 & -- & -- \\
\hline 2002 & 9 & 1 & 27.55 & 27.57 & 6.17 & 6.19 & 34.57 & 34.57 & -- & -- \\
\hline 2002 & 9 & 2 & 27.57 & 27.59 & 6.17 & 6.20 & 34.57 & 34.58 & -- & -- \\
\hline 2002 & 9 & 3 & 27.60 & 27.66 & 6.20 & 6.24 & 34.58 & 34.59 & -- & -- \\
\hline
\end{tabular}


Table 7. Daily mean and maximum depth, in feet, of water below land surface in four monitoring wells in Oakland County, Michigan, June 2001 through March 2004 - continued

$[--$, no data $]$

\begin{tabular}{|c|c|c|c|c|c|c|c|c|c|c|}
\hline \multirow[b]{2}{*}{ Year } & \multirow[b]{2}{*}{ Month } & \multirow[b]{2}{*}{ Day } & \multicolumn{2}{|c|}{$\begin{array}{c}\text { Holly State Rec. Area } \\
425116083321501\end{array}$} & \multicolumn{2}{|c|}{$\begin{array}{c}\text { Proud Lake State Rec. Area } \\
423423083324001\end{array}$} & \multicolumn{2}{|c|}{$\begin{array}{l}\text { Fish Lake Road } \\
424109083384301\end{array}$} & \multicolumn{2}{|c|}{$\begin{array}{l}\text { Teggerdine Road } \\
424133083293101\end{array}$} \\
\hline & & & Mean & Maximum & Mean & Maximum & Mean & Maximum & Mean & Maximum \\
\hline 2002 & 9 & 4 & 27.65 & 27.69 & 6.25 & 6.29 & 34.60 & 34.61 & -- & -- \\
\hline 2002 & 9 & 5 & 27.66 & 27.69 & 6.29 & 6.34 & 34.61 & 34.62 & -- & -- \\
\hline 2002 & 9 & 6 & 27.68 & 27.74 & 6.28 & 6.31 & 34.62 & 34.63 & -- & -- \\
\hline 2002 & 9 & 7 & 27.70 & 27.73 & 6.31 & 6.35 & 34.63 & 34.64 & -- & -- \\
\hline 2002 & 9 & 8 & 27.74 & 27.80 & 6.35 & 6.40 & 34.65 & 34.65 & -- & -- \\
\hline 2002 & 9 & 9 & 27.75 & 27.79 & 6.37 & 6.41 & 34.65 & 34.66 & -- & -- \\
\hline 2002 & 9 & 10 & 27.76 & 27.79 & 6.37 & 6.40 & 34.66 & 34.67 & -- & -- \\
\hline 2002 & 9 & 11 & 27.79 & 27.82 & 6.40 & 6.44 & 34.67 & 34.68 & -- & -- \\
\hline 2002 & 9 & 12 & 27.81 & 27.87 & 6.42 & 6.46 & 34.69 & 34.69 & -- & -- \\
\hline 2002 & 9 & 13 & 27.85 & 27.89 & 6.44 & 6.46 & 34.70 & 34.70 & -- & -- \\
\hline 2002 & 9 & 14 & 27.86 & 27.92 & 6.45 & 6.48 & 34.71 & 34.72 & -- & -- \\
\hline 2002 & 9 & 15 & 27.89 & 27.90 & 6.45 & 6.48 & 34.72 & 34.73 & -- & -- \\
\hline 2002 & 9 & 16 & 27.88 & 27.90 & 6.45 & 6.49 & 34.74 & 34.74 & -- & -- \\
\hline 2002 & 9 & 17 & 27.89 & 27.91 & 6.47 & 6.51 & 34.75 & 34.75 & -- & -- \\
\hline 2002 & 9 & 18 & 27.89 & 27.91 & 6.47 & 6.50 & 34.76 & 34.77 & -- & -- \\
\hline 2002 & 9 & 19 & 27.86 & 27.88 & 6.46 & 6.49 & 34.77 & 34.78 & -- & -- \\
\hline 2002 & 9 & 20 & 27.85 & 27.88 & 6.41 & 6.47 & 34.79 & 34.80 & -- & -- \\
\hline 2002 & 9 & 21 & 27.84 & 27.87 & 6.31 & 6.34 & 34.80 & 34.81 & -- & -- \\
\hline 2002 & 9 & 22 & 27.85 & 27.87 & 6.31 & 6.34 & 34.82 & 34.83 & -- & -- \\
\hline 2002 & 9 & 23 & 27.84 & 27.85 & 6.32 & 6.34 & 34.83 & 34.84 & -- & -- \\
\hline 2002 & 9 & 24 & 27.85 & 27.87 & 6.34 & 6.37 & 34.85 & 34.86 & -- & -- \\
\hline 2002 & 9 & 25 & 27.85 & 27.87 & 6.33 & 6.35 & 34.86 & 34.87 & -- & -- \\
\hline 2002 & 9 & 26 & 27.83 & 27.85 & 6.33 & 6.37 & 34.87 & 34.87 & -- & -- \\
\hline 2002 & 9 & 27 & 27.84 & 27.90 & 6.31 & 6.34 & 34.88 & 34.89 & -- & -- \\
\hline 2002 & 9 & 28 & 27.90 & 27.91 & 6.31 & 6.34 & 34.90 & 34.91 & -- & -- \\
\hline 2002 & 9 & 29 & 27.90 & 27.92 & 6.31 & 6.33 & 34.91 & 34.92 & -- & -- \\
\hline 2002 & 9 & 30 & 27.91 & 27.92 & 6.32 & 6.35 & 34.93 & 34.93 & -- & -- \\
\hline 2002 & 10 & 1 & 27.92 & 27.94 & 6.35 & 6.37 & 34.88 & 34.88 & -- & -- \\
\hline 2002 & 10 & 2 & 27.94 & 27.97 & 6.34 & 6.38 & 34.89 & 34.89 & -- & -- \\
\hline 2002 & 10 & 3 & 27.94 & 27.96 & 6.30 & 6.34 & 34.90 & 34.90 & -- & -- \\
\hline 2002 & 10 & 4 & 27.89 & 27.93 & 6.25 & 6.28 & 34.90 & 34.91 & 30.38 & 30.42 \\
\hline 2002 & 10 & 5 & 27.92 & 27.93 & 6.26 & 6.27 & 34.92 & 34.92 & 30.39 & 30.40 \\
\hline 2002 & 10 & 6 & 27.89 & 27.92 & 6.24 & 6.26 & 34.92 & 34.94 & 30.36 & 30.39 \\
\hline 2002 & 10 & 7 & 27.92 & 27.94 & 6.27 & 6.29 & 34.95 & 34.95 & 30.37 & 30.39 \\
\hline 2002 & 10 & 8 & 27.91 & 27.93 & 6.27 & 6.29 & 34.96 & 34.96 & 30.38 & 30.40 \\
\hline 2002 & 10 & 9 & 27.92 & 27.94 & 6.28 & 6.30 & 34.96 & 34.97 & 30.39 & 30.39 \\
\hline 2002 & 10 & 10 & 27.94 & 27.96 & 6.30 & 6.31 & 34.97 & 34.98 & 30.40 & 30.41 \\
\hline 2002 & 10 & 11 & 27.95 & 27.96 & 6.30 & 6.32 & 34.98 & 34.99 & 30.41 & 30.42 \\
\hline 2002 & 10 & 12 & 27.95 & 27.97 & 6.30 & 6.31 & 34.99 & 35.00 & 30.41 & 30.41 \\
\hline 2002 & 10 & 13 & 27.97 & 27.99 & 6.31 & 6.33 & 35.01 & 35.02 & 30.42 & 30.44 \\
\hline 2002 & 10 & 14 & 27.95 & 27.99 & 6.30 & 6.32 & 35.02 & 35.02 & 30.43 & 30.45 \\
\hline 2002 & 10 & 15 & 27.92 & 27.93 & 6.29 & 6.31 & 35.02 & 35.02 & 30.41 & 30.42 \\
\hline 2002 & 10 & 16 & 27.94 & 27.96 & 6.30 & 6.32 & 35.03 & 35.03 & 30.42 & 30.43 \\
\hline 2002 & 10 & 17 & 27.94 & 27.96 & 6.31 & 6.33 & 35.04 & 35.04 & 30.43 & 30.44 \\
\hline 2002 & 10 & 18 & 27.93 & 27.95 & 6.29 & 6.32 & 35.05 & 35.05 & 30.43 & 30.45 \\
\hline 2002 & 10 & 19 & 27.93 & 27.96 & 6.28 & 6.31 & 35.06 & 35.07 & 30.43 & 30.44 \\
\hline
\end{tabular}


Table 7. Daily mean and maximum depth, in feet, of water below land surface in four monitoring wells in Oakland County, Michigan, June 2001 through March 2004 - continued

$[--$, no data $]$

\begin{tabular}{|c|c|c|c|c|c|c|c|c|c|c|}
\hline \multirow[b]{2}{*}{ Year } & \multirow[b]{2}{*}{ Month } & \multirow[b]{2}{*}{ Day } & \multicolumn{2}{|c|}{$\begin{array}{c}\text { Holly State Rec. Area } \\
425116083321501\end{array}$} & \multicolumn{2}{|c|}{$\begin{array}{c}\text { Proud Lake State Rec. Area } \\
423423083324001\end{array}$} & \multicolumn{2}{|c|}{$\begin{array}{l}\text { Fish Lake Road } \\
424109083384301\end{array}$} & \multicolumn{2}{|c|}{$\begin{array}{l}\text { Teggerdine Road } \\
424133083293101\end{array}$} \\
\hline & & & Mean & Maximum & Mean & Maximum & Mean & Maximum & Mean & Maximum \\
\hline 2002 & 10 & 20 & 27.95 & 27.97 & 6.29 & 6.31 & 35.07 & 35.08 & 30.45 & 30.47 \\
\hline 2002 & 10 & 21 & 27.95 & 27.97 & 6.29 & 6.30 & 35.08 & 35.08 & 30.47 & 30.48 \\
\hline 2002 & 10 & 22 & 27.95 & 27.98 & 6.29 & 6.31 & 35.09 & 35.10 & 30.50 & 30.53 \\
\hline 2002 & 10 & 23 & 27.96 & 27.97 & 6.30 & 6.31 & 35.10 & 35.11 & 30.53 & 30.55 \\
\hline 2002 & 10 & 24 & 27.95 & 27.96 & 6.28 & 6.30 & 35.11 & 35.11 & 30.53 & 30.55 \\
\hline 2002 & 10 & 25 & 27.91 & 27.95 & 6.26 & 6.29 & 35.12 & 35.13 & 30.51 & 30.53 \\
\hline 2002 & 10 & 26 & 27.90 & 27.92 & 6.26 & 6.28 & 35.13 & 35.14 & 30.49 & 30.50 \\
\hline 2002 & 10 & 27 & 27.91 & 27.92 & 6.27 & 6.28 & 35.14 & 35.15 & 30.51 & 30.52 \\
\hline 2002 & 10 & 28 & 27.91 & 27.92 & 6.27 & 6.28 & 35.15 & 35.15 & 30.51 & 30.52 \\
\hline 2002 & 10 & 29 & 27.89 & 27.92 & 6.26 & 6.27 & 35.15 & 35.16 & 30.51 & 30.52 \\
\hline 2002 & 10 & 30 & 27.89 & 27.91 & 6.27 & 6.28 & 35.16 & 35.17 & 30.52 & 30.53 \\
\hline 2002 & 10 & 31 & -- & -- & 6.27 & 6.28 & 35.18 & 35.19 & 30.53 & 30.54 \\
\hline 2002 & 11 & 1 & -- & -- & 6.27 & 6.29 & 35.19 & 35.20 & 30.52 & 30.53 \\
\hline 2002 & 11 & 2 & -- & -- & 6.28 & 6.30 & 35.20 & 35.21 & 30.52 & 30.53 \\
\hline 2002 & 11 & 3 & -- & -- & 6.28 & 6.29 & 35.21 & 35.21 & 30.52 & 30.53 \\
\hline 2002 & 11 & 4 & -- & -- & 6.28 & 6.30 & 35.22 & 35.22 & 30.52 & 30.53 \\
\hline 2002 & 11 & 5 & -- & -- & 6.26 & 6.30 & 35.22 & 35.23 & 30.50 & 30.53 \\
\hline 2002 & 11 & 6 & -- & -- & 6.26 & 6.29 & 35.23 & 35.25 & 30.50 & 30.52 \\
\hline 2002 & 11 & 7 & -- & -- & 6.27 & 6.29 & 35.25 & 35.26 & 30.50 & 30.52 \\
\hline 2002 & 11 & 8 & -- & -- & 6.25 & 6.27 & 35.26 & 35.26 & 30.48 & 30.49 \\
\hline 2002 & 11 & 9 & -- & -- & 6.26 & 6.27 & 35.27 & 35.27 & 30.47 & 30.48 \\
\hline 2002 & 11 & 10 & -- & -- & 6.21 & 6.25 & 35.27 & 35.27 & 30.43 & 30.46 \\
\hline 2002 & 11 & 11 & 27.80 & 27.82 & 6.24 & 6.25 & 35.28 & 35.29 & 30.44 & 30.45 \\
\hline 2002 & 11 & 12 & 27.79 & 27.82 & 6.23 & 6.25 & 35.29 & 35.29 & 30.44 & 30.45 \\
\hline 2002 & 11 & 13 & 27.77 & 27.78 & 6.21 & 6.22 & 35.30 & 35.31 & 30.42 & 30.43 \\
\hline 2002 & 11 & 14 & 27.76 & 27.78 & 6.20 & 6.21 & 35.30 & 35.31 & 30.40 & 30.40 \\
\hline 2002 & 11 & 15 & 27.78 & 27.80 & 6.21 & 6.22 & 35.31 & 35.32 & 30.40 & 30.41 \\
\hline 2002 & 11 & 16 & 27.77 & 27.78 & 6.20 & 6.21 & 35.32 & 35.32 & 30.38 & 30.39 \\
\hline 2002 & 11 & 17 & 27.76 & 27.79 & 6.19 & 6.22 & 35.31 & 35.32 & 30.36 & 30.37 \\
\hline 2002 & 11 & 18 & 27.77 & 27.78 & 6.21 & 6.22 & 35.32 & 35.32 & 30.38 & 30.39 \\
\hline 2002 & 11 & 19 & 27.74 & 27.76 & 6.18 & 6.20 & 35.33 & 35.34 & 30.36 & 30.37 \\
\hline 2002 & 11 & 20 & 27.74 & 27.77 & 6.19 & 6.21 & 35.34 & 35.34 & 30.35 & 30.36 \\
\hline 2002 & 11 & 21 & 27.71 & 27.73 & 6.17 & 6.19 & 35.34 & 35.34 & 30.33 & 30.34 \\
\hline 2002 & 11 & 22 & 27.71 & 27.73 & 6.17 & 6.18 & 35.34 & 35.35 & 30.32 & 30.35 \\
\hline 2002 & 11 & 23 & 27.70 & 27.73 & 6.17 & 6.19 & 35.35 & 35.35 & 30.32 & 30.34 \\
\hline 2002 & 11 & 24 & 27.71 & 27.72 & 6.18 & 6.19 & 35.35 & 35.36 & 30.34 & 30.35 \\
\hline 2002 & 11 & 25 & 27.71 & 27.72 & 6.19 & 6.20 & 35.36 & 35.36 & 30.35 & 30.36 \\
\hline 2002 & 11 & 26 & 27.70 & 27.72 & 6.19 & 6.20 & 35.36 & 35.36 & 30.36 & 30.36 \\
\hline 2002 & 11 & 27 & 27.70 & 27.72 & 6.19 & 6.20 & 35.36 & 35.36 & 30.36 & 30.37 \\
\hline 2002 & 11 & 28 & 27.68 & 27.70 & 6.18 & 6.19 & 35.36 & 35.36 & 30.34 & 30.36 \\
\hline 2002 & 11 & 29 & 27.64 & 27.65 & 6.16 & 6.18 & 35.35 & 35.35 & 30.30 & 30.32 \\
\hline 2002 & 11 & 30 & 27.67 & 27.71 & 6.19 & 6.23 & 35.36 & 35.37 & 30.32 & 30.36 \\
\hline 2002 & 12 & 1 & 27.68 & 27.71 & 6.21 & 6.22 & 35.37 & 35.37 & 30.35 & 30.36 \\
\hline 2002 & 12 & 2 & 27.69 & 27.74 & 6.21 & 6.26 & 35.37 & 35.38 & 30.37 & 30.41 \\
\hline 2002 & 12 & 3 & -- & -- & 6.24 & 6.26 & 35.38 & 35.38 & 30.42 & 30.43 \\
\hline 2002 & 12 & 4 & 27.72 & 27.79 & 6.23 & 6.25 & 35.38 & 35.38 & 30.42 & 30.43 \\
\hline
\end{tabular}


Table 7. Daily mean and maximum depth, in feet, of water below land surface in four monitoring wells in Oakland County, Michigan, June 2001 through March 2004 - continued $[--$, no data $]$

\begin{tabular}{|c|c|c|c|c|c|c|c|c|c|c|}
\hline \multirow[b]{2}{*}{ Year } & \multirow[b]{2}{*}{ Month } & \multirow[b]{2}{*}{ Day } & \multicolumn{2}{|c|}{$\begin{array}{c}\text { Holly State Rec. Area } \\
425116083321501\end{array}$} & \multicolumn{2}{|c|}{$\begin{array}{l}\text { Proud Lake State Rec. Area } \\
\quad 423423083324001\end{array}$} & \multicolumn{2}{|c|}{$\begin{array}{l}\text { Fish Lake Road } \\
424109083384301\end{array}$} & \multicolumn{2}{|c|}{$\begin{array}{l}\text { Teggerdine Road } \\
424133083293101\end{array}$} \\
\hline & & & Mean & Maximum & Mean & Maximum & Mean & Maximum & Mean & Maximum \\
\hline 2002 & 12 & 5 & 27.67 & 27.69 & 6.21 & 6.23 & 35.38 & 35.38 & 30.40 & 30.41 \\
\hline 2002 & 12 & 6 & 27.68 & 27.69 & 6.22 & 6.23 & 35.38 & 35.39 & -- & -- \\
\hline 2002 & 12 & 7 & 27.68 & 27.69 & 6.22 & 6.24 & 35.39 & 35.39 & -- & -- \\
\hline 2002 & 12 & 8 & 27.71 & 27.75 & 6.25 & 6.27 & 35.39 & 35.40 & -- & -- \\
\hline 2002 & 12 & 9 & -- & -- & 6.24 & 6.26 & 35.40 & 35.40 & -- & -- \\
\hline 2002 & 12 & 10 & 27.69 & 27.69 & 6.24 & 6.25 & 35.40 & 35.40 & -- & -- \\
\hline 2002 & 12 & 11 & 27.69 & 27.72 & 6.24 & 6.25 & 35.40 & 35.40 & -- & -- \\
\hline 2002 & 12 & 12 & 27.69 & 27.71 & 6.25 & 6.26 & 35.41 & 35.41 & -- & -- \\
\hline 2002 & 12 & 13 & 27.67 & 27.69 & 6.23 & 6.25 & 35.40 & 35.41 & -- & -- \\
\hline 2002 & 12 & 14 & 27.68 & 27.70 & 6.22 & 6.24 & 35.41 & 35.41 & -- & -- \\
\hline 2002 & 12 & 15 & 27.68 & 27.70 & 6.22 & 6.24 & 35.41 & 35.42 & -- & -- \\
\hline 2002 & 12 & 16 & 27.71 & 27.73 & 6.24 & 6.25 & 35.42 & 35.43 & -- & -- \\
\hline 2002 & 12 & 17 & 27.70 & 27.72 & 6.23 & 6.24 & 35.43 & 35.43 & -- & -- \\
\hline 2002 & 12 & 18 & 27.67 & 27.69 & 6.21 & 6.24 & 35.43 & 35.43 & -- & -- \\
\hline 2002 & 12 & 19 & 27.62 & 27.66 & 6.16 & 6.20 & 35.43 & 35.43 & -- & -- \\
\hline 2002 & 12 & 20 & 27.57 & 27.59 & 6.14 & 6.15 & 35.43 & 35.44 & -- & -- \\
\hline 2002 & 12 & 21 & 27.58 & 27.59 & 6.15 & 6.18 & 35.44 & 35.44 & -- & -- \\
\hline 2002 & 12 & 22 & 27.58 & 27.61 & 6.15 & 6.17 & 35.44 & 35.45 & -- & -- \\
\hline 2002 & 12 & 23 & 27.59 & 27.61 & 6.16 & 6.18 & 35.45 & 35.45 & -- & -- \\
\hline 2002 & 12 & 24 & 27.57 & 27.61 & 6.15 & 6.18 & 35.45 & 35.45 & -- & -- \\
\hline 2002 & 12 & 25 & 27.53 & 27.57 & 6.15 & 6.18 & 35.45 & 35.45 & -- & -- \\
\hline 2002 & 12 & 26 & 27.55 & 27.57 & 6.17 & 6.18 & 35.46 & 35.46 & -- & -- \\
\hline 2002 & 12 & 27 & 27.52 & 27.55 & 6.16 & 6.18 & 35.46 & 35.46 & -- & -- \\
\hline 2002 & 12 & 28 & 27.48 & 27.50 & 6.14 & 6.16 & 35.46 & 35.47 & -- & -- \\
\hline 2002 & 12 & 29 & 27.48 & 27.50 & 6.16 & 6.17 & 35.47 & 35.47 & -- & -- \\
\hline 2002 & 12 & 30 & 27.42 & 27.47 & 6.13 & 6.16 & 35.47 & 35.47 & -- & -- \\
\hline 2002 & 12 & 31 & 27.39 & 27.41 & 6.12 & 6.14 & 35.48 & 35.48 & -- & -- \\
\hline 2003 & 1 & 1 & 27.37 & 27.40 & 6.12 & 6.14 & 35.48 & 35.48 & -- & -- \\
\hline 2003 & 1 & 2 & 27.35 & 27.37 & 6.12 & 6.14 & 35.48 & 35.49 & -- & -- \\
\hline 2003 & 1 & 3 & 27.33 & 27.37 & 6.12 & 6.13 & 35.49 & 35.49 & -- & -- \\
\hline 2003 & 1 & 4 & 27.32 & 27.35 & 6.12 & 6.14 & 35.49 & 35.50 & -- & -- \\
\hline 2003 & 1 & 5 & 27.30 & 27.33 & 6.12 & 6.14 & 35.50 & 35.50 & -- & -- \\
\hline 2003 & 1 & 6 & -- & -- & 6.14 & 6.15 & 35.50 & 35.51 & -- & -- \\
\hline 2003 & 1 & 7 & -- & -- & 6.11 & 6.14 & 35.50 & 35.51 & -- & -- \\
\hline 2003 & 1 & 8 & 27.19 & 27.22 & 6.10 & 6.12 & 35.50 & 35.51 & -- & -- \\
\hline 2003 & 1 & 9 & 27.19 & 27.22 & -- & -- & 35.51 & 35.52 & -- & -- \\
\hline 2003 & 1 & 10 & -- & -- & 6.13 & 6.15 & 35.52 & 35.53 & -- & -- \\
\hline 2003 & 1 & 11 & -- & -- & 6.15 & 6.18 & 35.53 & 35.54 & -- & -- \\
\hline 2003 & 1 & 12 & -- & -- & 6.16 & 6.17 & 35.54 & 35.54 & -- & -- \\
\hline 2003 & 1 & 13 & -- & -- & 6.15 & 6.18 & 35.54 & 35.55 & -- & -- \\
\hline 2003 & 1 & 14 & 27.17 & 27.19 & 6.16 & 6.17 & 35.55 & 35.55 & 30.36 & 30.37 \\
\hline 2003 & 1 & 15 & 27.19 & 27.21 & 6.17 & 6.19 & 35.56 & 35.56 & 30.37 & 30.39 \\
\hline 2003 & 1 & 16 & 27.20 & 27.23 & 6.16 & 6.19 & 35.56 & 35.57 & 30.37 & 30.39 \\
\hline 2003 & 1 & 17 & 27.22 & 27.25 & 6.18 & 6.19 & 35.57 & 35.57 & 30.39 & 30.40 \\
\hline 2003 & 1 & 18 & 27.22 & 27.25 & 6.17 & 6.19 & 35.57 & 35.58 & 30.38 & 30.39 \\
\hline 2003 & 1 & 19 & 27.23 & 27.26 & 6.18 & 6.20 & 35.58 & 35.59 & 30.38 & 30.39 \\
\hline
\end{tabular}


Table 7. Daily mean and maximum depth, in feet, of water below land surface in four monitoring wells in Oakland County, Michigan, June 2001 through March 2004 - continued $[--$, no data $]$

\begin{tabular}{|c|c|c|c|c|c|c|c|c|c|c|}
\hline \multirow[b]{2}{*}{ Year } & \multirow[b]{2}{*}{ Month } & \multirow[b]{2}{*}{ Day } & \multicolumn{2}{|c|}{$\begin{array}{c}\text { Holly State Rec. Area } \\
425116083321501\end{array}$} & \multicolumn{2}{|c|}{$\begin{array}{c}\text { Proud Lake State Rec. Area } \\
423423083324001\end{array}$} & \multicolumn{2}{|c|}{$\begin{array}{l}\text { Fish Lake Road } \\
424109083384301\end{array}$} & \multicolumn{2}{|c|}{$\begin{array}{l}\text { Teggerdine Road } \\
424133083293101\end{array}$} \\
\hline & & & Mean & Maximum & Mean & Maximum & Mean & Maximum & Mean & Maximum \\
\hline 2003 & 1 & 20 & 27.27 & 27.30 & 6.21 & 6.23 & 35.59 & 35.59 & 30.42 & 30.44 \\
\hline 2003 & 1 & 21 & 27.30 & 27.32 & 6.23 & 6.24 & 35.60 & 35.60 & 30.45 & 30.46 \\
\hline 2003 & 1 & 22 & 27.32 & 27.33 & 6.23 & 6.24 & 35.61 & 35.61 & 30.47 & 30.48 \\
\hline 2003 & 1 & 23 & 27.33 & 27.36 & 6.24 & 6.26 & 35.61 & 35.62 & 30.48 & 30.50 \\
\hline 2003 & 1 & 24 & 27.36 & 27.37 & 6.25 & 6.27 & 35.62 & 35.63 & 30.50 & 30.51 \\
\hline 2003 & 1 & 25 & 27.34 & 27.36 & 6.24 & 6.25 & 35.63 & 35.63 & 30.48 & 30.49 \\
\hline 2003 & 1 & 26 & 27.38 & 27.41 & 6.25 & 6.27 & 35.64 & 35.64 & 30.50 & 30.52 \\
\hline 2003 & 1 & 27 & 27.40 & 27.41 & 6.26 & 6.26 & 35.65 & 35.65 & 30.51 & 30.52 \\
\hline 2003 & 1 & 28 & 27.37 & 27.39 & 6.24 & 6.26 & 35.65 & 35.66 & 30.49 & 30.50 \\
\hline 2003 & 1 & 29 & 27.42 & 27.44 & 6.26 & 6.28 & 35.66 & 35.67 & 30.51 & 30.53 \\
\hline 2003 & 1 & 30 & 27.42 & 27.43 & 6.26 & 6.27 & 35.66 & 35.67 & 30.51 & 30.52 \\
\hline 2003 & 1 & 31 & 27.40 & 27.43 & 6.25 & 6.26 & 35.67 & 35.67 & 30.49 & 30.51 \\
\hline 2003 & 2 & 1 & 27.40 & 27.42 & 6.25 & 6.26 & 35.67 & 35.68 & 30.48 & 30.49 \\
\hline 2003 & 2 & 2 & 27.42 & 27.44 & 6.26 & 6.26 & 35.68 & 35.69 & 30.49 & 30.51 \\
\hline 2003 & 2 & 3 & 27.38 & 27.41 & 6.22 & 6.26 & 35.68 & 35.69 & 30.46 & 30.50 \\
\hline 2003 & 2 & 4 & 27.37 & 27.42 & 6.20 & 6.23 & 35.70 & 35.70 & 30.41 & 30.43 \\
\hline 2003 & 2 & 5 & 27.42 & 27.43 & 6.21 & 6.24 & 35.70 & 35.71 & 30.43 & 30.44 \\
\hline 2003 & 2 & 6 & 27.40 & 27.42 & 6.19 & 6.20 & 35.70 & 35.71 & 30.42 & 30.43 \\
\hline 2003 & 2 & 7 & 27.40 & 27.41 & 6.17 & 6.18 & 35.71 & 35.71 & 30.40 & 30.41 \\
\hline 2003 & 2 & 8 & 27.39 & 27.42 & 6.17 & 6.18 & 35.71 & 35.72 & 30.38 & 30.39 \\
\hline 2003 & 2 & 9 & 27.42 & 27.45 & 6.18 & 6.20 & 35.72 & 35.72 & 30.39 & 30.40 \\
\hline 2003 & 2 & 10 & 27.42 & 27.47 & 6.17 & 6.21 & 35.72 & 35.73 & 30.38 & 30.40 \\
\hline 2003 & 2 & 11 & 27.42 & 27.46 & 6.17 & 6.20 & 35.72 & 35.73 & 30.38 & 30.40 \\
\hline 2003 & 2 & 12 & 27.47 & 27.49 & 6.20 & 6.22 & 35.73 & 35.73 & 30.41 & 30.43 \\
\hline 2003 & 2 & 13 & 27.48 & 27.51 & 6.20 & 6.23 & 35.74 & 35.74 & 30.44 & 30.45 \\
\hline 2003 & 2 & 14 & 27.50 & 27.51 & 6.21 & 6.23 & 35.74 & 35.75 & 30.45 & 30.47 \\
\hline 2003 & 2 & 15 & 27.53 & 27.57 & 6.24 & 6.25 & 35.75 & 35.76 & 30.49 & 30.51 \\
\hline 2003 & 2 & 16 & 27.54 & 27.56 & 6.22 & 6.24 & 35.76 & 35.76 & 30.49 & 30.50 \\
\hline 2003 & 2 & 17 & 27.52 & 27.54 & 6.21 & 6.22 & 35.76 & 35.77 & 30.47 & 30.48 \\
\hline 2003 & 2 & 18 & 27.53 & 27.55 & 6.22 & 6.24 & 35.76 & 35.77 & 30.47 & 30.48 \\
\hline 2003 & 2 & 19 & 27.56 & 27.57 & 6.23 & 6.24 & 35.77 & 35.78 & 30.48 & 30.50 \\
\hline 2003 & 2 & 20 & 27.56 & 27.58 & 6.23 & 6.24 & 35.78 & 35.78 & 30.49 & 30.50 \\
\hline 2003 & 2 & 21 & 27.54 & 27.56 & 6.21 & 6.22 & 35.79 & 35.79 & 30.47 & 30.48 \\
\hline 2003 & 2 & 22 & 27.52 & 27.55 & 6.19 & 6.20 & 35.79 & 35.79 & 30.42 & 30.45 \\
\hline 2003 & 2 & 23 & 27.55 & 27.59 & 6.22 & 6.25 & 35.80 & 35.80 & 30.44 & 30.47 \\
\hline 2003 & 2 & 24 & 27.57 & 27.59 & 6.23 & 6.24 & 35.80 & 35.81 & 30.47 & 30.48 \\
\hline 2003 & 2 & 25 & 27.58 & 27.60 & 6.23 & 6.24 & 35.81 & 35.81 & 30.49 & 30.50 \\
\hline 2003 & 2 & 26 & 27.54 & 27.58 & 6.21 & 6.22 & 35.82 & 35.82 & 30.47 & 30.48 \\
\hline 2003 & 2 & 27 & 27.53 & 27.55 & 6.20 & 6.23 & 35.82 & 35.83 & 30.47 & 30.47 \\
\hline 2003 & 2 & 28 & 27.54 & 27.56 & 6.22 & 6.23 & 35.83 & 35.84 & 30.47 & 30.49 \\
\hline 2003 & 3 & 1 & 27.53 & 27.55 & 6.21 & 6.23 & 35.83 & 35.84 & 30.47 & 30.49 \\
\hline 2003 & 3 & 2 & 27.53 & 27.57 & 6.21 & 6.25 & 35.84 & 35.84 & 30.45 & 30.48 \\
\hline 2003 & 3 & 3 & 27.55 & 27.58 & 6.22 & 6.25 & 35.84 & 35.85 & 30.48 & 30.49 \\
\hline 2003 & 3 & 4 & 27.52 & 27.54 & 6.21 & 6.23 & 35.85 & 35.86 & 30.46 & 30.48 \\
\hline 2003 & 3 & 5 & 27.53 & 27.55 & 6.22 & 6.23 & 35.86 & 35.87 & 30.47 & 30.49 \\
\hline 2003 & 3 & 6 & 27.55 & 27.57 & 6.23 & 6.23 & 35.87 & 35.88 & 30.49 & 30.51 \\
\hline
\end{tabular}


Table 7. Daily mean and maximum depth, in feet, of water below land surface in four monitoring wells in Oakland County, Michigan, June 2001 through March 2004 - continued $[--$, no data $]$

\begin{tabular}{|c|c|c|c|c|c|c|c|c|c|c|}
\hline \multirow[b]{2}{*}{ Year } & \multirow[b]{2}{*}{ Month } & \multirow[b]{2}{*}{ Day } & \multicolumn{2}{|c|}{$\begin{array}{c}\text { Holly State Rec. Area } \\
425116083321501\end{array}$} & \multicolumn{2}{|c|}{$\begin{array}{c}\text { Proud Lake State Rec. Area } \\
423423083324001\end{array}$} & \multicolumn{2}{|c|}{$\begin{array}{l}\text { Fish Lake Road } \\
424109083384301\end{array}$} & \multicolumn{2}{|c|}{$\begin{array}{l}\text { Teggerdine Road } \\
424133083293101\end{array}$} \\
\hline & & & Mean & Maximum & Mean & Maximum & Mean & Maximum & Mean & Maximum \\
\hline 2003 & 3 & 7 & 27.53 & 27.56 & 6.22 & 6.23 & 35.87 & 35.88 & 30.48 & 30.50 \\
\hline 2003 & 3 & 8 & 27.51 & 27.53 & 6.21 & 6.23 & 35.88 & 35.89 & 30.46 & 30.49 \\
\hline 2003 & 3 & 9 & 27.54 & 27.56 & 6.21 & 6.23 & 35.89 & 35.89 & 30.48 & 30.49 \\
\hline 2003 & 3 & 10 & 27.54 & 27.56 & 6.20 & 6.22 & 35.90 & 35.90 & 30.49 & 30.50 \\
\hline 2003 & 3 & 11 & 27.52 & 27.54 & 6.19 & 6.21 & 35.90 & 35.91 & 30.47 & 30.48 \\
\hline 2003 & 3 & 12 & 27.53 & 27.56 & 6.20 & 6.22 & 35.91 & 35.92 & 30.47 & 30.48 \\
\hline 2003 & 3 & 13 & 27.54 & 27.55 & 6.20 & 6.22 & 35.92 & 35.93 & 30.48 & 30.50 \\
\hline 2003 & 3 & 14 & 27.52 & 27.56 & 6.19 & 6.21 & 35.93 & 35.95 & 30.47 & 30.49 \\
\hline 2003 & 3 & 15 & 27.50 & 27.52 & 6.17 & 6.18 & 35.95 & 35.95 & 30.44 & 30.45 \\
\hline 2003 & 3 & 16 & 27.46 & 27.50 & 6.11 & 6.14 & 35.94 & 35.95 & 30.36 & 30.40 \\
\hline 2003 & 3 & 17 & 27.38 & 27.42 & 6.05 & 6.08 & 35.90 & 35.93 & 30.24 & 30.28 \\
\hline 2003 & 3 & 18 & 27.33 & 27.35 & 6.01 & 6.03 & 35.80 & 35.85 & 30.15 & 30.18 \\
\hline 2003 & 3 & 19 & 27.28 & 27.32 & 5.98 & 6.01 & 35.72 & 35.75 & 30.09 & 30.12 \\
\hline 2003 & 3 & 20 & 27.24 & 27.27 & 5.95 & 5.97 & 35.67 & 35.69 & 30.04 & 30.06 \\
\hline 2003 & 3 & 21 & 27.21 & 27.23 & 5.91 & 5.93 & 35.66 & 35.66 & 29.98 & 30.00 \\
\hline 2003 & 3 & 22 & 27.21 & 27.23 & 5.91 & 5.93 & 35.66 & 35.67 & 29.96 & 29.97 \\
\hline 2003 & 3 & 23 & 27.22 & 27.24 & 5.90 & 5.93 & 35.67 & 35.68 & 29.94 & 29.96 \\
\hline 2003 & 3 & 24 & 27.19 & 27.21 & 5.89 & 5.90 & 35.68 & 35.68 & 29.93 & 29.93 \\
\hline 2003 & 3 & 25 & 27.16 & 27.18 & 5.88 & 5.89 & 35.69 & 35.69 & 29.92 & 29.92 \\
\hline 2003 & 3 & 26 & 27.15 & 27.16 & 5.87 & 5.88 & 35.70 & 35.70 & 29.91 & 29.91 \\
\hline 2003 & 3 & 27 & 27.13 & 27.15 & 5.87 & 5.88 & 35.71 & 35.71 & 29.91 & 29.92 \\
\hline 2003 & 3 & 28 & 27.10 & 27.12 & 5.84 & 5.86 & 35.72 & 35.72 & 29.89 & 29.90 \\
\hline 2003 & 3 & 29 & 27.10 & 27.12 & 5.84 & 5.86 & 35.73 & 35.74 & 29.87 & 29.88 \\
\hline 2003 & 3 & 30 & 27.08 & 27.11 & 5.84 & 5.86 & 35.75 & 35.75 & 29.87 & 29.88 \\
\hline 2003 & 3 & 31 & 27.07 & 27.08 & 5.82 & 5.84 & 35.76 & 35.77 & 29.86 & 29.87 \\
\hline 2003 & 4 & 1 & 27.05 & 27.08 & 5.81 & 5.84 & 35.77 & 35.78 & 29.83 & 29.86 \\
\hline 2003 & 4 & 2 & 27.07 & 27.08 & 5.83 & 5.85 & 35.78 & 35.79 & 29.86 & 29.87 \\
\hline 2003 & 4 & 3 & 27.06 & 27.08 & 5.82 & 5.84 & 35.80 & 35.81 & 29.86 & 29.87 \\
\hline 2003 & 4 & 4 & 26.91 & 27.03 & 5.73 & 5.80 & 35.81 & 35.81 & 29.77 & 29.85 \\
\hline 2003 & 4 & 5 & 26.85 & 26.88 & 5.70 & 5.73 & 35.82 & 35.82 & 29.67 & 29.69 \\
\hline 2003 & 4 & 6 & 26.86 & 26.88 & 5.69 & 5.72 & 35.81 & 35.82 & 29.65 & 29.67 \\
\hline 2003 & 4 & 7 & 26.81 & 26.83 & 5.64 & 5.66 & 35.78 & 35.79 & 29.60 & 29.62 \\
\hline 2003 & 4 & 8 & 26.83 & 26.85 & 5.63 & 5.64 & 35.76 & 35.76 & 29.60 & 29.60 \\
\hline 2003 & 4 & 9 & 26.82 & 26.85 & 5.61 & 5.63 & 35.75 & 35.76 & 29.59 & 29.60 \\
\hline 2003 & 4 & 10 & 26.78 & 26.81 & 5.58 & 5.60 & 35.74 & 35.75 & 29.56 & 29.57 \\
\hline 2003 & 4 & 11 & 26.76 & 26.77 & 5.56 & 5.58 & 35.74 & 35.74 & 29.53 & 29.54 \\
\hline 2003 & 4 & 12 & 26.78 & 26.81 & 5.57 & 5.59 & 35.74 & 35.75 & 29.54 & 29.57 \\
\hline 2003 & 4 & 13 & 26.79 & 26.81 & 5.58 & 5.58 & 35.75 & 35.75 & 29.58 & 29.59 \\
\hline 2003 & 4 & 14 & 26.77 & 26.80 & 5.56 & 5.58 & 35.75 & 35.75 & 29.58 & 29.58 \\
\hline 2003 & 4 & 15 & 26.77 & 26.78 & 5.54 & 5.56 & 35.75 & 35.75 & 29.57 & 29.57 \\
\hline 2003 & 4 & 16 & 26.79 & 26.83 & 5.56 & 5.58 & 35.76 & 35.77 & 29.59 & 29.61 \\
\hline 2003 & 4 & 17 & 26.79 & 26.81 & 5.55 & 5.57 & 35.78 & 35.79 & 29.61 & 29.62 \\
\hline 2003 & 4 & 18 & 26.79 & 26.82 & 5.56 & 5.58 & 35.80 & 35.81 & 29.63 & 29.65 \\
\hline 2003 & 4 & 19 & 26.80 & 26.82 & 5.56 & 5.57 & 35.81 & 35.82 & 29.66 & 29.66 \\
\hline 2003 & 4 & 20 & 26.77 & 26.78 & 5.54 & 5.56 & 35.82 & 35.83 & 29.63 & 29.65 \\
\hline 2003 & 4 & 21 & 26.77 & 26.79 & 5.52 & 5.54 & 35.83 & 35.84 & 29.55 & 29.58 \\
\hline
\end{tabular}


Table 7. Daily mean and maximum depth, in feet, of water below land surface in four monitoring wells in Oakland County, Michigan, June 2001 through March 2004 - continued $[--$, no data $]$

\begin{tabular}{|c|c|c|c|c|c|c|c|c|c|c|}
\hline \multirow[b]{2}{*}{ Year } & \multirow[b]{2}{*}{ Month } & \multirow[b]{2}{*}{ Day } & \multicolumn{2}{|c|}{$\begin{array}{c}\text { Holly State Rec. Area } \\
425116083321501\end{array}$} & \multicolumn{2}{|c|}{$\begin{array}{c}\text { Proud Lake State Rec. Area } \\
423423083324001\end{array}$} & \multicolumn{2}{|c|}{$\begin{array}{l}\text { Fish Lake Road } \\
424109083384301\end{array}$} & \multicolumn{2}{|c|}{$\begin{array}{l}\text { Teggerdine Road } \\
424133083293101\end{array}$} \\
\hline & & & Mean & Maximum & Mean & Maximum & Mean & Maximum & Mean & Maximum \\
\hline 2003 & 4 & 22 & 26.79 & 26.81 & 5.53 & 5.54 & 35.85 & 35.86 & 29.54 & 29.56 \\
\hline 2003 & 4 & 23 & 26.82 & 26.86 & 5.53 & 5.54 & 35.86 & 35.87 & 29.57 & 29.58 \\
\hline 2003 & 4 & 24 & 26.81 & 26.83 & 5.52 & 5.53 & 35.87 & 35.88 & 29.57 & 29.58 \\
\hline 2003 & 4 & 25 & 26.80 & 26.83 & 5.53 & 5.55 & 35.88 & 35.89 & 29.55 & 29.56 \\
\hline 2003 & 4 & 26 & 26.83 & 26.86 & 5.53 & 5.56 & 35.90 & 35.90 & 29.57 & 29.59 \\
\hline 2003 & 4 & 27 & 26.84 & 26.86 & 5.54 & 5.56 & 35.91 & 35.91 & 29.60 & 29.61 \\
\hline 2003 & 4 & 28 & 26.84 & 26.88 & 5.54 & 5.57 & 35.92 & 35.93 & 29.61 & 29.63 \\
\hline 2003 & 4 & 29 & 26.88 & 26.88 & 5.57 & 5.59 & 35.93 & 35.94 & 29.64 & 29.65 \\
\hline 2003 & 4 & 30 & 26.86 & 26.89 & 5.56 & 5.59 & 35.94 & 35.95 & 29.65 & 29.66 \\
\hline 2003 & 5 & 1 & 26.78 & 26.84 & 5.52 & 5.54 & 35.96 & 35.96 & 29.62 & 29.64 \\
\hline 2003 & 5 & 2 & 26.72 & 26.75 & 5.52 & 5.54 & 35.97 & 35.98 & 29.62 & 29.63 \\
\hline 2003 & 5 & 3 & 26.72 & 26.73 & 5.52 & 5.54 & 35.98 & 35.99 & 29.63 & 29.64 \\
\hline 2003 & 5 & 4 & 26.70 & 26.73 & 5.52 & 5.54 & 35.99 & 35.99 & 29.62 & 29.64 \\
\hline 2003 & 5 & 5 & 26.65 & 26.68 & 5.48 & 5.51 & 35.99 & 36.00 & 29.56 & 29.59 \\
\hline 2003 & 5 & 6 & 26.62 & 26.64 & 5.49 & 5.51 & 36.01 & 36.01 & 29.57 & 29.58 \\
\hline 2003 & 5 & 7 & 26.62 & 26.64 & 5.50 & 5.52 & 36.01 & 36.02 & 29.58 & 29.59 \\
\hline 2003 & 5 & 8 & 26.63 & 26.65 & 5.50 & 5.52 & 36.03 & 36.03 & 29.59 & 29.61 \\
\hline 2003 & 5 & 9 & 26.65 & 26.70 & 5.48 & 5.51 & 36.03 & 36.04 & 29.58 & 29.60 \\
\hline 2003 & 5 & 10 & 26.67 & 26.69 & 5.46 & 5.48 & 36.05 & 36.05 & 29.55 & 29.56 \\
\hline 2003 & 5 & 11 & 26.65 & 26.67 & 5.44 & 5.46 & 36.06 & 36.06 & 29.48 & 29.51 \\
\hline 2003 & 5 & 12 & 26.69 & 26.73 & 5.45 & 5.47 & 36.06 & 36.07 & 29.47 & 29.48 \\
\hline 2003 & 5 & 13 & 26.71 & 26.73 & 5.45 & 5.47 & 36.07 & 36.08 & 29.47 & 29.48 \\
\hline 2003 & 5 & 14 & 26.73 & 26.74 & 5.45 & 5.46 & 36.08 & 36.09 & 29.47 & 29.48 \\
\hline 2003 & 5 & 15 & 26.74 & 26.76 & 5.44 & 5.46 & 36.09 & 36.10 & 29.47 & 29.48 \\
\hline 2003 & 5 & 16 & 26.77 & 26.78 & 5.44 & 5.46 & 36.10 & 36.11 & 29.49 & 29.50 \\
\hline 2003 & 5 & 17 & 26.79 & 26.83 & 5.45 & 5.47 & 36.11 & 36.12 & 29.52 & 29.53 \\
\hline 2003 & 5 & 18 & 26.79 & 26.81 & 5.47 & 5.49 & 36.12 & 36.13 & 29.54 & 29.55 \\
\hline 2003 & 5 & 19 & 26.81 & 26.84 & 5.48 & 5.50 & 36.13 & 36.13 & 29.55 & 29.57 \\
\hline 2003 & 5 & 20 & 26.83 & 26.87 & 5.49 & 5.51 & 36.14 & 36.14 & 29.56 & 29.58 \\
\hline 2003 & 5 & 21 & 26.88 & 26.90 & 5.51 & 5.53 & 36.15 & 36.15 & 29.59 & 29.60 \\
\hline 2003 & 5 & 22 & 26.88 & 26.92 & 5.53 & 5.55 & 36.16 & 36.16 & 29.60 & 29.61 \\
\hline 2003 & 5 & 23 & 26.88 & 26.90 & 5.52 & 5.54 & 36.17 & 36.17 & 29.60 & 29.60 \\
\hline 2003 & 5 & 24 & 26.89 & 26.91 & 5.52 & 5.55 & 36.18 & 36.18 & 29.59 & 29.60 \\
\hline 2003 & 5 & 25 & 26.93 & 26.97 & 5.55 & 5.57 & 36.19 & 36.19 & 29.62 & 29.63 \\
\hline 2003 & 5 & 26 & 26.95 & 26.97 & 5.57 & 5.58 & 36.19 & 36.20 & 29.64 & 29.65 \\
\hline 2003 & 5 & 27 & 26.94 & 26.96 & 5.58 & 5.59 & 36.20 & 36.21 & 29.66 & 29.67 \\
\hline 2003 & 5 & 28 & 26.92 & 26.95 & 5.58 & 5.59 & 36.21 & 36.22 & 29.65 & 29.67 \\
\hline 2003 & 5 & 29 & 26.94 & 26.96 & 5.60 & 5.64 & 36.22 & 36.23 & 29.66 & 29.67 \\
\hline 2003 & 5 & 30 & 26.93 & 26.95 & 5.62 & 5.65 & 36.23 & 36.24 & 29.67 & 29.68 \\
\hline 2003 & 5 & 31 & 26.89 & 26.92 & 5.59 & 5.62 & 36.25 & 36.25 & 29.65 & 29.67 \\
\hline 2003 & 6 & 1 & 26.88 & 26.90 & 5.60 & 5.62 & 36.25 & 36.26 & 29.67 & 29.68 \\
\hline 2003 & 6 & 2 & 26.89 & 26.91 & 5.62 & 5.65 & 36.26 & 36.27 & 29.68 & 29.69 \\
\hline 2003 & 6 & 3 & 26.89 & 26.91 & 5.63 & 5.65 & 36.27 & 36.27 & 29.67 & 29.68 \\
\hline 2003 & 6 & 4 & 26.89 & 26.91 & 5.61 & 5.64 & 36.28 & 36.28 & 29.67 & 29.68 \\
\hline 2003 & 6 & 5 & 26.91 & 26.93 & 5.60 & 5.62 & 36.29 & 36.30 & 29.65 & 29.66 \\
\hline 2003 & 6 & 6 & 26.92 & 26.95 & 5.61 & 5.62 & 36.30 & 36.31 & 29.66 & 29.66 \\
\hline
\end{tabular}


Table 7. Daily mean and maximum depth, in feet, of water below land surface in four monitoring wells in Oakland County, Michigan, June 2001 through March 2004 - continued $[--$, no data $]$

\begin{tabular}{|c|c|c|c|c|c|c|c|c|c|c|}
\hline \multirow[b]{2}{*}{ Year } & \multirow[b]{2}{*}{ Month } & \multirow[b]{2}{*}{ Day } & \multicolumn{2}{|c|}{$\begin{array}{c}\text { Holly State Rec. Area } \\
425116083321501\end{array}$} & \multicolumn{2}{|c|}{$\begin{array}{c}\text { Proud Lake State Rec. Area } \\
423423083324001\end{array}$} & \multicolumn{2}{|c|}{$\begin{array}{l}\text { Fish Lake Road } \\
424109083384301\end{array}$} & \multicolumn{2}{|c|}{$\begin{array}{l}\text { Teggerdine Road } \\
424133083293101\end{array}$} \\
\hline & & & Mean & Maximum & Mean & Maximum & Mean & Maximum & Mean & Maximum \\
\hline 2003 & 6 & 7 & 26.93 & 26.96 & 5.62 & 5.65 & 36.31 & 36.31 & 29.66 & 29.67 \\
\hline 2003 & 6 & 8 & 26.90 & 26.94 & 5.63 & 5.65 & 36.32 & 36.32 & 29.66 & 29.68 \\
\hline 2003 & 6 & 9 & 26.79 & 26.81 & 5.63 & 5.65 & 36.32 & 36.33 & 29.65 & 29.66 \\
\hline 2003 & 6 & 10 & 26.76 & 26.80 & 5.63 & 5.66 & 36.33 & 36.33 & 29.65 & 29.66 \\
\hline 2003 & 6 & 11 & 26.77 & 26.79 & 5.64 & 5.66 & 36.34 & 36.34 & 29.66 & 29.68 \\
\hline 2003 & 6 & 12 & 26.70 & 26.78 & 5.62 & 5.65 & 36.34 & 36.35 & 29.65 & 29.67 \\
\hline 2003 & 6 & 13 & 26.62 & 26.64 & 5.61 & 5.64 & 36.35 & 36.36 & 29.62 & 29.63 \\
\hline 2003 & 6 & 14 & 26.65 & 26.68 & 5.63 & 5.66 & 36.36 & 36.36 & 29.63 & 29.65 \\
\hline 2003 & 6 & 15 & 26.68 & 26.73 & 5.68 & 5.71 & 36.35 & 36.35 & 29.67 & 29.68 \\
\hline 2003 & 6 & 16 & 26.71 & 26.73 & 5.71 & 5.73 & 36.35 & 36.36 & 29.70 & 29.72 \\
\hline 2003 & 6 & 17 & 26.71 & 26.73 & 5.71 & 5.74 & 36.36 & 36.36 & 29.71 & 29.72 \\
\hline 2003 & 6 & 18 & 26.71 & 26.73 & 5.69 & 5.72 & 36.36 & 36.37 & 29.72 & 29.74 \\
\hline 2003 & 6 & 19 & 26.76 & 26.79 & 5.60 & 5.61 & 36.37 & 36.37 & 29.72 & 29.75 \\
\hline 2003 & 6 & 20 & 26.78 & 26.80 & 5.60 & 5.63 & 36.37 & 36.38 & 29.75 & 29.76 \\
\hline 2003 & 6 & 21 & 26.83 & 26.88 & 5.62 & 5.64 & 36.38 & 36.38 & 29.77 & 29.79 \\
\hline 2003 & 6 & 22 & 26.85 & 26.89 & 5.64 & 5.67 & 36.39 & 36.39 & 29.80 & 29.81 \\
\hline 2003 & 6 & 23 & 26.91 & 26.97 & 5.68 & 5.71 & 36.39 & 36.39 & 29.83 & 29.85 \\
\hline 2003 & 6 & 24 & 26.97 & 27.03 & 5.74 & 5.76 & 36.40 & 36.40 & 29.87 & 29.89 \\
\hline 2003 & 6 & 25 & 27.03 & 27.07 & 5.75 & 5.76 & 36.40 & 36.41 & 29.90 & 29.92 \\
\hline 2003 & 6 & 26 & 27.04 & 27.07 & 5.75 & 5.77 & 36.41 & 36.41 & -- & -- \\
\hline 2003 & 6 & 27 & 27.08 & 27.11 & 5.77 & 5.79 & 36.42 & 36.42 & 29.95 & 29.97 \\
\hline 2003 & 6 & 28 & 27.11 & 27.16 & 5.78 & 5.82 & 36.42 & 36.43 & 29.97 & 29.98 \\
\hline 2003 & 6 & 29 & 27.13 & 27.17 & 5.77 & 5.79 & 36.43 & 36.43 & 30.00 & 30.02 \\
\hline 2003 & 6 & 30 & 27.17 & 27.22 & 5.81 & 5.83 & 36.43 & 36.44 & 30.04 & 30.06 \\
\hline 2003 & 7 & 1 & 27.20 & 27.25 & 5.84 & 5.87 & 36.44 & 36.44 & 30.07 & 30.08 \\
\hline 2003 & 7 & 2 & 27.22 & 27.27 & 5.88 & 5.92 & 36.44 & 36.45 & 30.09 & 30.10 \\
\hline 2003 & 7 & 3 & 27.27 & 27.28 & 5.92 & 5.93 & 36.45 & 36.46 & 30.12 & 30.14 \\
\hline 2003 & 7 & 4 & 27.30 & 27.34 & 5.91 & 5.95 & 36.46 & 36.47 & 30.15 & 30.16 \\
\hline 2003 & 7 & 5 & 27.33 & 27.35 & 5.88 & 5.90 & 36.47 & 36.47 & 30.17 & 30.18 \\
\hline 2003 & 7 & 6 & 27.34 & 27.36 & 5.88 & 5.91 & 36.47 & 36.48 & 30.18 & 30.19 \\
\hline 2003 & 7 & 7 & 27.36 & 27.42 & 5.89 & 5.91 & 36.48 & 36.48 & 30.19 & 30.20 \\
\hline 2003 & 7 & 8 & 27.37 & 27.38 & 5.93 & 5.95 & 36.49 & 36.49 & 30.21 & 30.22 \\
\hline 2003 & 7 & 9 & 27.38 & 27.41 & 5.91 & 5.93 & 36.49 & 36.49 & 30.23 & 30.24 \\
\hline 2003 & 7 & 10 & 27.37 & 27.39 & 5.90 & 5.92 & 36.49 & 36.49 & 30.21 & 30.23 \\
\hline 2003 & 7 & 11 & 27.39 & 27.42 & 5.90 & 5.91 & 36.50 & 36.50 & 30.20 & 30.22 \\
\hline 2003 & 7 & 12 & 27.42 & 27.45 & 5.92 & 5.95 & 36.51 & 36.51 & 30.24 & 30.26 \\
\hline 2003 & 7 & 13 & 27.46 & 27.52 & 5.97 & 5.99 & 36.51 & 36.52 & 30.27 & 30.28 \\
\hline 2003 & 7 & 14 & 27.47 & 27.51 & 6.00 & 6.03 & 36.52 & 36.52 & 30.29 & 30.30 \\
\hline 2003 & 7 & 15 & 27.47 & 27.49 & 6.01 & 6.03 & 36.53 & 36.53 & 30.29 & 30.31 \\
\hline 2003 & 7 & 16 & 27.53 & 27.59 & 6.06 & 6.08 & 36.54 & 36.54 & 30.32 & 30.33 \\
\hline 2003 & 7 & 17 & 27.55 & 27.57 & 6.07 & 6.09 & 36.54 & 36.55 & 30.35 & 30.37 \\
\hline 2003 & 7 & 18 & 27.59 & 27.62 & 6.09 & 6.12 & 36.55 & 36.56 & 30.37 & 30.39 \\
\hline 2003 & 7 & 19 & 27.63 & 27.67 & 6.13 & 6.17 & 36.56 & 36.56 & 30.40 & 30.41 \\
\hline 2003 & 7 & 20 & 27.63 & 27.67 & 6.15 & 6.16 & 36.57 & 36.57 & 30.41 & 30.42 \\
\hline 2003 & 7 & 21 & 27.59 & 27.67 & 6.11 & 6.13 & 36.57 & 36.58 & 30.41 & 30.43 \\
\hline 2003 & 7 & 22 & 27.48 & 27.50 & 6.11 & 6.13 & 36.58 & 36.59 & 30.40 & 30.41 \\
\hline
\end{tabular}


Table 7. Daily mean and maximum depth, in feet, of water below land surface in four monitoring wells in Oakland County, Michigan, June 2001 through March 2004 - continued

$[--$, no data $]$

\begin{tabular}{|c|c|c|c|c|c|c|c|c|c|c|}
\hline \multirow[b]{2}{*}{ Year } & \multirow[b]{2}{*}{ Month } & \multirow[b]{2}{*}{ Day } & \multicolumn{2}{|c|}{$\begin{array}{c}\text { Holly State Rec. Area } \\
425116083321501\end{array}$} & \multicolumn{2}{|c|}{$\begin{array}{l}\text { Proud Lake State Rec. Area } \\
\quad 423423083324001\end{array}$} & \multicolumn{2}{|c|}{$\begin{array}{l}\text { Fish Lake Road } \\
424109083384301\end{array}$} & \multicolumn{2}{|c|}{$\begin{array}{l}\text { Teggerdine Road } \\
424133083293101\end{array}$} \\
\hline & & & Mean & Maximum & Mean & Maximum & Mean & Maximum & Mean & Maximum \\
\hline 2003 & 7 & 23 & 27.46 & 27.47 & 6.14 & 6.17 & 36.59 & 36.60 & 30.42 & 30.43 \\
\hline 2003 & 7 & 24 & 27.49 & 27.55 & 6.19 & 6.23 & 36.60 & 36.61 & 30.44 & 30.46 \\
\hline 2003 & 7 & 25 & 27.52 & 27.56 & 6.23 & 6.25 & 36.61 & 36.61 & 30.46 & 30.47 \\
\hline 2003 & 7 & 26 & 27.54 & 27.58 & 6.23 & 6.26 & 36.62 & 36.62 & 30.47 & 30.49 \\
\hline 2003 & 7 & 27 & 27.55 & 27.60 & 6.24 & 6.29 & 36.63 & 36.63 & 30.49 & 30.52 \\
\hline 2003 & 7 & 28 & 27.61 & 27.67 & 6.28 & 6.31 & 36.63 & 36.64 & 30.53 & 30.54 \\
\hline 2003 & 7 & 29 & 27.64 & 27.67 & 6.31 & 6.36 & 36.64 & 36.65 & 30.55 & 30.56 \\
\hline 2003 & 7 & 30 & 27.66 & 27.69 & 6.35 & 6.37 & 36.65 & 36.66 & 30.57 & 30.59 \\
\hline 2003 & 7 & 31 & 27.69 & 27.74 & 6.37 & 6.38 & 36.66 & 36.67 & 30.60 & 30.61 \\
\hline 2003 & 8 & 1 & 27.71 & 27.74 & 6.38 & 6.41 & 36.67 & 36.68 & 30.61 & 30.64 \\
\hline 2003 & 8 & 2 & 27.72 & 27.74 & 6.33 & 6.34 & 36.68 & 36.69 & 30.59 & 30.60 \\
\hline 2003 & 8 & 3 & 27.72 & 27.74 & 6.34 & 6.35 & 36.69 & 36.69 & 30.59 & 30.59 \\
\hline 2003 & 8 & 4 & 27.67 & 27.69 & 6.31 & 6.33 & 36.70 & 36.70 & 30.59 & 30.60 \\
\hline 2003 & 8 & 5 & 27.66 & 27.68 & 6.32 & 6.34 & 36.71 & 36.71 & 30.60 & 30.62 \\
\hline 2003 & 8 & 6 & 27.68 & 27.71 & 6.36 & 6.38 & 36.71 & 36.72 & 30.62 & 30.63 \\
\hline 2003 & 8 & 7 & 27.71 & 27.73 & 6.39 & 6.41 & 36.72 & 36.73 & 30.64 & 30.66 \\
\hline 2003 & 8 & 8 & 27.73 & 27.74 & 6.41 & 6.43 & 36.73 & 36.74 & 30.67 & 30.69 \\
\hline 2003 & 8 & 9 & 27.73 & 27.75 & 6.44 & 6.47 & 36.74 & 36.75 & 30.69 & 30.71 \\
\hline 2003 & 8 & 10 & 27.71 & 27.72 & 6.48 & 6.50 & 36.75 & 36.75 & 30.71 & 30.72 \\
\hline 2003 & 8 & 11 & 27.72 & 27.75 & 6.47 & 6.50 & 36.76 & 36.76 & 30.72 & 30.74 \\
\hline 2003 & 8 & 12 & 27.74 & 27.77 & 6.48 & 6.51 & 36.77 & 36.77 & 30.74 & 30.75 \\
\hline 2003 & 8 & 13 & 27.73 & 27.74 & 6.44 & 6.46 & 36.78 & 36.78 & 30.75 & 30.76 \\
\hline 2003 & 8 & 14 & 27.73 & 27.75 & 6.47 & 6.50 & 36.78 & 36.79 & 30.76 & 30.77 \\
\hline 2003 & 8 & 15 & 27.71 & 27.74 & 6.41 & 6.44 & 36.79 & 36.79 & 30.73 & 30.76 \\
\hline 2003 & 8 & 16 & 27.73 & 27.78 & 6.39 & 6.41 & 36.79 & 36.80 & 30.64 & 30.67 \\
\hline 2003 & 8 & 17 & 27.78 & 27.83 & 6.43 & 6.46 & 36.79 & 36.80 & 30.63 & 30.63 \\
\hline 2003 & 8 & 18 & 27.80 & 27.84 & 6.48 & 6.50 & 36.80 & 36.81 & 30.64 & 30.66 \\
\hline 2003 & 8 & 19 & 27.83 & 27.86 & 6.52 & 6.54 & 36.81 & 36.82 & 30.66 & 30.67 \\
\hline 2003 & 8 & 20 & 27.85 & 27.91 & 6.55 & 6.57 & 36.82 & 36.83 & 30.68 & 30.70 \\
\hline 2003 & 8 & 21 & 27.87 & 27.90 & 6.57 & 6.59 & 36.83 & 36.84 & 30.69 & 30.70 \\
\hline 2003 & 8 & 22 & 27.89 & 27.91 & 6.58 & 6.60 & 36.84 & 36.85 & 30.71 & 30.72 \\
\hline 2003 & 8 & 23 & 27.91 & 27.93 & 6.63 & 6.65 & 36.85 & 36.86 & 30.74 & 30.75 \\
\hline 2003 & 8 & 24 & 27.91 & 27.94 & 6.64 & 6.66 & 36.86 & 36.86 & 30.75 & 30.76 \\
\hline 2003 & 8 & 25 & 27.93 & 27.95 & 6.65 & 6.67 & 36.87 & 36.87 & 30.77 & 30.78 \\
\hline 2003 & 8 & 26 & 27.91 & 27.94 & 6.61 & 6.65 & 36.88 & 36.88 & 30.77 & 30.78 \\
\hline 2003 & 8 & 27 & 27.92 & 27.99 & 6.62 & 6.66 & 36.89 & 36.89 & 30.77 & 30.78 \\
\hline 2003 & 8 & 28 & 27.93 & 27.95 & 6.66 & 6.68 & 36.89 & 36.90 & 30.78 & 30.79 \\
\hline 2003 & 8 & 29 & 27.94 & 27.98 & 6.68 & 6.70 & 36.91 & 36.91 & 30.79 & 30.82 \\
\hline 2003 & 8 & 30 & 27.99 & 28.01 & 6.71 & 6.73 & 36.92 & 36.92 & 30.83 & 30.84 \\
\hline 2003 & 8 & 31 & 27.99 & 28.00 & 6.72 & 6.73 & 36.92 & 36.93 & 30.84 & 30.85 \\
\hline 2003 & 9 & 1 & 27.99 & 28.00 & 6.68 & 6.71 & 36.93 & 36.94 & 30.84 & 30.85 \\
\hline 2003 & 9 & 2 & 27.98 & 28.01 & 6.64 & 6.65 & 36.94 & 36.95 & 30.84 & 30.85 \\
\hline 2003 & 9 & 3 & 27.99 & 28.01 & 6.66 & 6.67 & 36.95 & 36.96 & 30.85 & 30.85 \\
\hline 2003 & 9 & 4 & 28.01 & 28.03 & 6.68 & 6.70 & 36.96 & 36.96 & 30.85 & 30.86 \\
\hline 2003 & 9 & 5 & 28.03 & 28.05 & 6.70 & 6.71 & 36.97 & 36.98 & 30.87 & 30.88 \\
\hline 2003 & 9 & 6 & 28.08 & 28.14 & 6.72 & 6.74 & 36.98 & 36.98 & 30.89 & 30.90 \\
\hline
\end{tabular}


Table 7. Daily mean and maximum depth, in feet, of water below land surface in four monitoring wells in Oakland County, Michigan, June 2001 through March 2004 - continued

$[--$, no data $]$

\begin{tabular}{|c|c|c|c|c|c|c|c|c|c|c|}
\hline \multirow[b]{2}{*}{ Year } & \multirow[b]{2}{*}{ Month } & \multirow[b]{2}{*}{ Day } & \multicolumn{2}{|c|}{$\begin{array}{c}\text { Holly State Rec. Area } \\
425116083321501\end{array}$} & \multicolumn{2}{|c|}{$\begin{array}{c}\text { Proud Lake State Rec. Area } \\
423423083324001\end{array}$} & \multicolumn{2}{|c|}{$\begin{array}{l}\text { Fish Lake Road } \\
424109083384301\end{array}$} & \multicolumn{2}{|c|}{$\begin{array}{l}\text { Teggerdine Road } \\
424133083293101\end{array}$} \\
\hline & & & Mean & Maximum & Mean & Maximum & Mean & Maximum & Mean & Maximum \\
\hline 2003 & 9 & 7 & 28.08 & 28.10 & 6.75 & 6.78 & 36.99 & 36.99 & 30.91 & 30.92 \\
\hline 2003 & 9 & 8 & 28.09 & 28.11 & 6.77 & 6.79 & 37.00 & 37.00 & 30.93 & 30.94 \\
\hline 2003 & 9 & 9 & 28.11 & 28.14 & -- & -- & 37.01 & 37.01 & 30.95 & 30.96 \\
\hline 2003 & 9 & 10 & 28.12 & 28.17 & -- & -- & 37.02 & 37.02 & 30.96 & 30.98 \\
\hline 2003 & 9 & 11 & 28.15 & 28.19 & -- & -- & 37.03 & 37.03 & 30.98 & 30.99 \\
\hline 2003 & 9 & 12 & 28.15 & 28.18 & -- & -- & 37.04 & 37.04 & 30.99 & 31.01 \\
\hline 2003 & 9 & 13 & 28.18 & 28.23 & -- & -- & 37.05 & 37.05 & 31.01 & 31.02 \\
\hline 2003 & 9 & 14 & 28.20 & 28.21 & -- & -- & 37.05 & 37.06 & 31.03 & 31.03 \\
\hline 2003 & 9 & 15 & 28.19 & 28.20 & -- & -- & 37.06 & 37.07 & 31.03 & 31.03 \\
\hline 2003 & 9 & 16 & 28.18 & 28.19 & -- & -- & 37.07 & 37.08 & 31.04 & 31.05 \\
\hline 2003 & 9 & 17 & 28.19 & 28.22 & -- & -- & 37.08 & 37.09 & 31.05 & 31.06 \\
\hline 2003 & 9 & 18 & 28.19 & 28.21 & -- & -- & 37.09 & 37.10 & 31.06 & 31.06 \\
\hline 2003 & 9 & 19 & 28.18 & 28.21 & -- & -- & 37.10 & 37.11 & 31.05 & 31.06 \\
\hline 2003 & 9 & 20 & 28.21 & 28.23 & -- & -- & 37.11 & 37.12 & 31.07 & 31.08 \\
\hline 2003 & 9 & 21 & 28.20 & 28.22 & -- & -- & 37.12 & 37.12 & 31.08 & 31.08 \\
\hline 2003 & 9 & 22 & 28.13 & 28.20 & -- & -- & 37.13 & 37.13 & 31.02 & 31.07 \\
\hline 2003 & 9 & 23 & 28.05 & 28.07 & -- & -- & 37.14 & 37.14 & 30.96 & 30.97 \\
\hline 2003 & 9 & 24 & 28.01 & 28.05 & -- & -- & 37.15 & 37.15 & 30.93 & 30.95 \\
\hline 2003 & 9 & 25 & 27.97 & 28.00 & -- & -- & 37.16 & 37.16 & 30.89 & 30.92 \\
\hline 2003 & 9 & 26 & 27.92 & 27.96 & -- & -- & 37.16 & 37.17 & 30.86 & 30.88 \\
\hline 2003 & 9 & 27 & 27.89 & 27.90 & -- & -- & 37.17 & 37.18 & 30.84 & 30.85 \\
\hline 2003 & 9 & 28 & 27.89 & 27.91 & -- & -- & 37.18 & 37.18 & 30.84 & 30.85 \\
\hline 2003 & 9 & 29 & 27.90 & 27.92 & -- & -- & 37.18 & 37.19 & 30.86 & 30.86 \\
\hline 2003 & 9 & 30 & 27.90 & 27.91 & -- & -- & 37.19 & 37.19 & 30.86 & 30.86 \\
\hline 2003 & 10 & 1 & 27.90 & 27.92 & -- & -- & 37.20 & 37.20 & 30.86 & 30.86 \\
\hline 2003 & 10 & 2 & 27.90 & 27.92 & 6.63 & 6.64 & 37.21 & 37.21 & 30.86 & 30.87 \\
\hline 2003 & 10 & 3 & 27.87 & 27.90 & 6.60 & 6.63 & 37.21 & 37.22 & 30.83 & 30.86 \\
\hline 2003 & 10 & 4 & 27.88 & 27.89 & 6.60 & 6.62 & 37.22 & 37.23 & 30.83 & 30.84 \\
\hline 2003 & 10 & 5 & 27.89 & 27.91 & 6.62 & 6.63 & 37.23 & 37.23 & 30.84 & 30.85 \\
\hline 2003 & 10 & 6 & 27.90 & 27.92 & 6.62 & 6.64 & 37.23 & 37.24 & 30.85 & 30.86 \\
\hline 2003 & 10 & 7 & 27.89 & 27.91 & 6.62 & 6.64 & 37.24 & 37.25 & 30.85 & 30.86 \\
\hline 2003 & 10 & 8 & 27.89 & 27.91 & 6.63 & 6.65 & 37.25 & 37.25 & 30.86 & 30.86 \\
\hline 2003 & 10 & 9 & 27.89 & 27.91 & 6.64 & 6.67 & 37.26 & 37.26 & 30.86 & 30.87 \\
\hline 2003 & 10 & 10 & 27.87 & 27.89 & 6.65 & 6.66 & 37.26 & 37.27 & 30.86 & 30.87 \\
\hline 2003 & 10 & 11 & 27.87 & 27.89 & 6.64 & 6.65 & 37.27 & 37.28 & 30.87 & 30.88 \\
\hline 2003 & 10 & 12 & 27.86 & 27.89 & 6.64 & 6.67 & 37.28 & 37.29 & 30.87 & 30.88 \\
\hline 2003 & 10 & 13 & 27.86 & 27.88 & 6.65 & 6.66 & 37.29 & 37.29 & 30.88 & 30.88 \\
\hline 2003 & 10 & 14 & 27.82 & 27.85 & 6.59 & 6.64 & 37.29 & 37.30 & 30.83 & 30.87 \\
\hline 2003 & 10 & 15 & 27.80 & 27.82 & 6.53 & 6.54 & 37.30 & 37.31 & 30.80 & 30.81 \\
\hline 2003 & 10 & 16 & 27.80 & 27.80 & 6.52 & 6.53 & 37.31 & 37.32 & 30.79 & 30.79 \\
\hline 2003 & 10 & 17 & 27.79 & 27.80 & 6.52 & 6.53 & 37.32 & 37.32 & 30.79 & 30.79 \\
\hline 2003 & 10 & 18 & 27.78 & 27.80 & 6.50 & 6.51 & 37.32 & 37.33 & 30.77 & 30.78 \\
\hline 2003 & 10 & 19 & 27.82 & 27.84 & 6.51 & 6.52 & 37.33 & 37.34 & 30.77 & 30.78 \\
\hline 2003 & 10 & 20 & 27.79 & 27.83 & 6.49 & 6.51 & 37.34 & 37.34 & 30.75 & 30.77 \\
\hline 2003 & 10 & 21 & 27.78 & 27.80 & 6.50 & 6.51 & 37.35 & 37.35 & 30.74 & 30.75 \\
\hline 2003 & 10 & 22 & 27.77 & 27.80 & 6.50 & 6.51 & 37.35 & 37.36 & 30.75 & 30.76 \\
\hline
\end{tabular}


Table 7. Daily mean and maximum depth, in feet, of water below land surface in four monitoring wells in Oakland County, Michigan, June 2001 through March 2004 - continued $[--$, no data $]$

\begin{tabular}{|c|c|c|c|c|c|c|c|c|c|c|}
\hline \multirow[b]{2}{*}{ Year } & \multirow[b]{2}{*}{ Month } & \multirow[b]{2}{*}{ Day } & \multicolumn{2}{|c|}{$\begin{array}{c}\text { Holly State Rec. Area } \\
425116083321501\end{array}$} & \multicolumn{2}{|c|}{$\begin{array}{c}\text { Proud Lake State Rec. Area } \\
423423083324001\end{array}$} & \multicolumn{2}{|c|}{$\begin{array}{l}\text { Fish Lake Road } \\
424109083384301\end{array}$} & \multicolumn{2}{|c|}{$\begin{array}{l}\text { Teggerdine Road } \\
424133083293101\end{array}$} \\
\hline & & & Mean & Maximum & Mean & Maximum & Mean & Maximum & Mean & Maximum \\
\hline 2003 & 10 & 23 & 27.77 & 27.80 & 6.50 & 6.51 & 37.36 & 37.36 & 30.77 & 30.78 \\
\hline 2003 & 10 & 24 & 27.78 & 27.80 & 6.51 & 6.51 & 37.37 & 37.37 & 30.79 & 30.80 \\
\hline 2003 & 10 & 25 & 27.76 & 27.79 & 6.48 & 6.51 & 37.38 & 37.39 & 30.77 & 30.80 \\
\hline 2003 & 10 & 26 & 27.73 & 27.75 & 6.44 & 6.46 & 37.39 & 37.39 & 30.74 & 30.76 \\
\hline 2003 & 10 & 27 & 27.70 & 27.73 & 6.43 & 6.45 & 37.39 & 37.40 & 30.72 & 30.74 \\
\hline 2003 & 10 & 28 & 27.69 & 27.71 & 6.42 & 6.44 & 37.40 & 37.41 & 30.70 & 30.71 \\
\hline 2003 & 10 & 29 & 27.70 & 27.71 & 6.43 & 6.45 & 37.41 & 37.41 & 30.71 & 30.73 \\
\hline 2003 & 10 & 30 & 27.70 & 27.73 & 6.42 & 6.45 & 37.42 & 37.42 & 30.73 & 30.74 \\
\hline 2003 & 10 & 31 & 27.70 & 27.72 & 6.43 & 6.44 & 37.42 & 37.43 & 30.73 & 30.74 \\
\hline 2003 & 11 & 1 & 27.70 & 27.72 & 6.43 & 6.44 & 37.43 & 37.43 & 30.75 & 30.75 \\
\hline 2003 & 11 & 2 & 27.63 & 27.70 & 6.37 & 6.42 & 37.44 & 37.44 & 30.70 & 30.74 \\
\hline 2003 & 11 & 3 & 27.53 & 27.59 & 6.33 & 6.36 & 37.44 & 37.45 & 30.65 & 30.67 \\
\hline 2003 & 11 & 4 & 27.45 & 27.48 & 6.30 & 6.32 & 37.45 & 37.46 & 30.60 & 30.62 \\
\hline 2003 & 11 & 5 & 27.46 & 27.48 & 6.31 & 6.33 & 37.46 & 37.46 & 30.58 & 30.58 \\
\hline 2003 & 11 & 6 & 27.44 & 27.47 & 6.30 & 6.32 & 37.46 & 37.46 & 30.56 & 30.58 \\
\hline 2003 & 11 & 7 & 27.44 & 27.47 & 6.29 & 6.31 & 37.46 & 37.46 & 30.55 & 30.57 \\
\hline 2003 & 11 & 8 & 27.46 & 27.48 & 6.31 & 6.33 & 37.46 & 37.46 & 30.57 & 30.58 \\
\hline 2003 & 11 & 9 & 27.45 & 27.47 & 6.31 & 6.33 & 37.47 & 37.47 & 30.56 & 30.58 \\
\hline 2003 & 11 & 10 & 27.42 & 27.45 & 6.28 & 6.30 & 37.47 & 37.47 & 30.55 & 30.56 \\
\hline 2003 & 11 & 11 & 27.39 & 27.42 & 6.24 & 6.27 & 37.47 & 37.47 & 30.51 & 30.53 \\
\hline 2003 & 11 & 12 & 27.38 & 27.41 & 6.23 & 6.26 & 37.47 & 37.48 & 30.48 & 30.50 \\
\hline 2003 & 11 & 13 & 27.43 & 27.46 & 6.26 & 6.28 & 37.48 & 37.48 & 30.51 & 30.53 \\
\hline 2003 & 11 & 14 & 27.42 & 27.46 & 6.25 & 6.27 & 37.48 & 37.49 & 30.51 & 30.52 \\
\hline 2003 & 11 & 15 & 27.42 & 27.43 & 6.25 & 6.26 & 37.49 & 37.49 & 30.51 & 30.52 \\
\hline 2003 & 11 & 16 & 27.41 & 27.42 & 6.24 & 6.26 & 37.49 & 37.49 & 30.52 & 30.52 \\
\hline 2003 & 11 & 17 & 27.41 & 27.42 & 6.24 & 6.26 & 37.50 & 37.50 & 30.53 & 30.53 \\
\hline 2003 & 11 & 18 & 27.33 & 27.40 & 6.18 & 6.23 & 37.50 & 37.50 & 30.46 & 30.51 \\
\hline 2003 & 11 & 19 & 27.27 & 27.30 & 6.16 & 6.19 & 37.50 & 37.51 & 30.40 & 30.42 \\
\hline 2003 & 11 & 20 & 27.25 & 27.28 & 6.16 & 6.19 & 37.51 & 37.51 & 30.38 & 30.39 \\
\hline 2003 & 11 & 21 & 27.25 & 27.28 & 6.16 & 6.18 & 37.51 & 37.51 & 30.37 & 30.38 \\
\hline 2003 & 11 & 22 & 27.24 & 27.27 & 6.15 & 6.17 & 37.51 & 37.51 & 30.36 & 30.37 \\
\hline 2003 & 11 & 23 & 27.22 & 27.24 & 6.13 & 6.16 & 37.51 & 37.51 & 30.34 & 30.36 \\
\hline 2003 & 11 & 24 & 27.18 & 27.20 & 6.11 & 6.14 & 37.51 & 37.52 & 30.31 & 30.32 \\
\hline 2003 & 11 & 25 & 27.17 & 27.20 & 6.11 & 6.13 & 37.52 & 37.52 & 30.31 & 30.32 \\
\hline 2003 & 11 & 26 & 27.16 & 27.18 & 6.11 & 6.12 & 37.52 & 37.52 & 30.31 & 30.31 \\
\hline 2003 & 11 & 27 & 27.15 & 27.18 & 6.09 & 6.11 & 37.52 & 37.52 & 30.30 & 30.31 \\
\hline 2003 & 11 & 28 & 27.12 & 27.16 & 6.06 & 6.10 & 37.53 & 37.53 & 30.27 & 30.30 \\
\hline 2003 & 11 & 29 & 27.09 & 27.12 & 6.05 & 6.07 & 37.53 & 37.53 & 30.26 & 30.27 \\
\hline 2003 & 11 & 30 & 27.07 & 27.09 & 6.04 & 6.05 & 37.53 & 37.53 & 30.24 & 30.24 \\
\hline 2003 & 12 & 1 & 27.10 & 27.12 & 6.06 & 6.07 & 37.53 & 37.53 & 30.26 & 30.28 \\
\hline 2003 & 12 & 2 & 27.10 & 27.12 & 6.06 & 6.07 & 37.53 & 37.53 & 30.28 & 30.29 \\
\hline 2003 & 12 & 3 & 27.09 & 27.11 & 6.05 & 6.07 & 37.53 & 37.53 & 30.28 & 30.29 \\
\hline 2003 & 12 & 4 & 27.06 & 27.09 & 6.03 & 6.04 & 37.53 & 37.53 & 30.26 & 30.27 \\
\hline 2003 & 12 & 5 & 27.06 & 27.09 & 6.03 & 6.05 & 37.53 & 37.53 & 30.25 & 30.27 \\
\hline 2003 & 12 & 6 & 27.07 & 27.10 & 6.03 & 6.05 & 37.52 & 37.52 & 30.27 & 30.27 \\
\hline 2003 & 12 & 7 & 27.06 & 27.09 & 6.03 & 6.05 & 37.51 & 37.52 & 30.27 & 30.28 \\
\hline
\end{tabular}


Table 7. Daily mean and maximum depth, in feet, of water below land surface in four monitoring wells in Oakland County, Michigan, June 2001 through March 2004 - continued $[--$, no data $]$

\begin{tabular}{|c|c|c|c|c|c|c|c|c|c|c|}
\hline \multirow[b]{2}{*}{ Year } & \multirow[b]{2}{*}{ Month } & \multirow[b]{2}{*}{ Day } & \multicolumn{2}{|c|}{$\begin{array}{c}\text { Holly State Rec. Area } \\
425116083321501\end{array}$} & \multicolumn{2}{|c|}{$\begin{array}{c}\text { Proud Lake State Rec. Area } \\
423423083324001\end{array}$} & \multicolumn{2}{|c|}{$\begin{array}{l}\text { Fish Lake Road } \\
424109083384301\end{array}$} & \multicolumn{2}{|c|}{$\begin{array}{l}\text { Teggerdine Road } \\
424133083293101\end{array}$} \\
\hline & & & Mean & Maximum & Mean & Maximum & Mean & Maximum & Mean & Maximum \\
\hline 2003 & 12 & 8 & 27.06 & 27.08 & 6.03 & 6.04 & 37.50 & 37.51 & 30.28 & 30.29 \\
\hline 2003 & 12 & 9 & 27.05 & 27.08 & 6.02 & 6.04 & 37.50 & 37.50 & 30.28 & 30.29 \\
\hline 2003 & 12 & 10 & 27.01 & 27.05 & 5.99 & 6.02 & 37.49 & 37.49 & 30.24 & 30.28 \\
\hline 2003 & 12 & 11 & 27.04 & 27.08 & 6.00 & 6.02 & 37.49 & 37.50 & 30.24 & 30.26 \\
\hline 2003 & 12 & 12 & 27.05 & 27.07 & 6.01 & 6.02 & 37.49 & 37.49 & 30.27 & 30.28 \\
\hline 2003 & 12 & 13 & 27.04 & 27.08 & 6.01 & 6.02 & 37.48 & 37.48 & 30.28 & 30.29 \\
\hline 2003 & 12 & 14 & 26.99 & 27.02 & 5.98 & 6.00 & 37.47 & 37.47 & 30.24 & 30.26 \\
\hline 2003 & 12 & 15 & 27.01 & 27.04 & 6.01 & 6.02 & 37.47 & 37.47 & 30.25 & 30.26 \\
\hline 2003 & 12 & 16 & 26.98 & 27.00 & 5.99 & 6.01 & 37.46 & 37.47 & 30.23 & 30.24 \\
\hline 2003 & 12 & 17 & 26.98 & 27.00 & 5.99 & 6.00 & 37.46 & 37.46 & 30.23 & 30.24 \\
\hline 2003 & 12 & 18 & 26.98 & 27.00 & 5.99 & 6.01 & 37.46 & 37.46 & 30.23 & 30.25 \\
\hline 2003 & 12 & 19 & 26.95 & 26.97 & 5.99 & 6.02 & 37.45 & 37.45 & 30.25 & 30.27 \\
\hline 2003 & 12 & 20 & 26.95 & 26.97 & 6.01 & 6.03 & 37.45 & 37.45 & 30.29 & 30.30 \\
\hline 2003 & 12 & 21 & 26.90 & 26.93 & 5.99 & 6.01 & 37.45 & 37.45 & 30.28 & 30.29 \\
\hline 2003 & 12 & 22 & 26.89 & 26.92 & 5.99 & 6.00 & 37.44 & 37.45 & 30.27 & 30.28 \\
\hline 2003 & 12 & 23 & 26.86 & 26.90 & 5.95 & 5.99 & 37.44 & 37.44 & 30.24 & 30.28 \\
\hline 2003 & 12 & 24 & 26.82 & 26.85 & 5.95 & 5.97 & 37.44 & 37.44 & 30.22 & 30.23 \\
\hline 2003 & 12 & 25 & 26.81 & 26.83 & 5.95 & 5.97 & 37.43 & 37.44 & 30.21 & 30.22 \\
\hline 2003 & 12 & 26 & 26.82 & 26.85 & 5.96 & 5.98 & 37.43 & 37.43 & 30.23 & 30.23 \\
\hline 2003 & 12 & 27 & 26.79 & 26.81 & 5.95 & 5.97 & 37.42 & 37.43 & 30.22 & 30.23 \\
\hline 2003 & 12 & 28 & 26.76 & 26.78 & 5.92 & 5.94 & 37.42 & 37.42 & 30.19 & 30.20 \\
\hline 2003 & 12 & 29 & 26.69 & 26.76 & 5.89 & 5.92 & 37.42 & 37.42 & 30.15 & 30.18 \\
\hline 2003 & 12 & 30 & 26.65 & 26.67 & 5.89 & 5.91 & 37.41 & 37.42 & 30.13 & 30.13 \\
\hline 2003 & 12 & 31 & 26.63 & 26.67 & 5.88 & 5.90 & 37.41 & 37.41 & 30.09 & 30.11 \\
\hline 2004 & 1 & 1 & 26.63 & 26.67 & 5.88 & 5.90 & 37.40 & 37.40 & 30.08 & 30.10 \\
\hline 2004 & 1 & 2 & 26.56 & 26.59 & 5.84 & 5.85 & 37.39 & 37.39 & 30.03 & 30.05 \\
\hline 2004 & 1 & 3 & 26.57 & 26.60 & 5.84 & 5.86 & 37.39 & 37.39 & 30.02 & 30.04 \\
\hline 2004 & 1 & 4 & 26.58 & 26.60 & 5.84 & 5.86 & 37.38 & 37.39 & 30.02 & 30.03 \\
\hline 2004 & 1 & 5 & 26.57 & 26.60 & 5.85 & 5.87 & 37.38 & 37.38 & 30.01 & 30.03 \\
\hline 2004 & 1 & 6 & 26.59 & 26.62 & -- & -- & 37.37 & 37.37 & 30.03 & 30.04 \\
\hline 2004 & 1 & 7 & 26.59 & 26.60 & -- & -- & 37.36 & 37.37 & 30.04 & 30.05 \\
\hline 2004 & 1 & 8 & 26.58 & 26.60 & -- & -- & 37.35 & 37.36 & 30.04 & 30.05 \\
\hline 2004 & 1 & 9 & 26.61 & 26.64 & -- & -- & 37.34 & 37.35 & 30.06 & 30.08 \\
\hline 2004 & 1 & 10 & 26.60 & 26.64 & -- & -- & 37.34 & 37.34 & 30.06 & 30.08 \\
\hline 2004 & 1 & 11 & 26.55 & 26.57 & -- & -- & 37.33 & 37.34 & 30.02 & 30.05 \\
\hline 2004 & 1 & 12 & 26.57 & 26.59 & -- & -- & 37.33 & 37.33 & 30.04 & 30.06 \\
\hline 2004 & 1 & 13 & 26.59 & 26.62 & 5.81 & 5.83 & 37.32 & 37.33 & 30.07 & 30.09 \\
\hline 2004 & 1 & 14 & 26.53 & 26.59 & 5.77 & 5.81 & 37.31 & 37.32 & 30.04 & 30.08 \\
\hline 2004 & 1 & 15 & 26.59 & 26.61 & 5.80 & 5.82 & 37.31 & 37.31 & 30.08 & 30.10 \\
\hline 2004 & 1 & 16 & 26.58 & 26.61 & 5.80 & 5.81 & 37.30 & 37.31 & 30.11 & 30.12 \\
\hline 2004 & 1 & 17 & 26.52 & 26.58 & 5.76 & 5.79 & 37.29 & 37.30 & 30.07 & 30.11 \\
\hline 2004 & 1 & 18 & 26.53 & 26.59 & 5.77 & 5.80 & 37.29 & 37.29 & 30.06 & 30.09 \\
\hline 2004 & 1 & 19 & 26.57 & 26.59 & 5.79 & 5.82 & 37.29 & 37.29 & 30.10 & 30.12 \\
\hline 2004 & 1 & 20 & 26.58 & 26.62 & 5.79 & 5.81 & 37.28 & 37.28 & 30.13 & 30.14 \\
\hline 2004 & 1 & 21 & 26.53 & 26.59 & 5.76 & 5.80 & 37.27 & 37.28 & 30.10 & 30.14 \\
\hline 2004 & 1 & 22 & 26.56 & 26.59 & 5.78 & 5.81 & 37.27 & 37.28 & 30.11 & 30.14 \\
\hline
\end{tabular}


Table 7. Daily mean and maximum depth, in feet, of water below land surface in four monitoring wells in Oakland County, Michigan, June 2001 through March 2004 - continued $[--$, no data $]$

\begin{tabular}{|c|c|c|c|c|c|c|c|c|c|c|}
\hline \multirow[b]{2}{*}{ Year } & \multirow[b]{2}{*}{ Month } & \multirow[b]{2}{*}{ Day } & \multicolumn{2}{|c|}{$\begin{array}{c}\text { Holly State Rec. Area } \\
425116083321501\end{array}$} & \multicolumn{2}{|c|}{$\begin{array}{c}\text { Proud Lake State Rec. Area } \\
423423083324001\end{array}$} & \multicolumn{2}{|c|}{$\begin{array}{l}\text { Fish Lake Road } \\
424109083384301\end{array}$} & \multicolumn{2}{|c|}{$\begin{array}{l}\text { Teggerdine Road } \\
424133083293101\end{array}$} \\
\hline & & & Mean & Maximum & Mean & Maximum & Mean & Maximum & Mean & Maximum \\
\hline 2004 & 1 & 23 & 26.56 & 26.59 & 5.76 & 5.79 & 37.27 & 37.27 & 30.12 & 30.13 \\
\hline 2004 & 1 & 24 & 26.58 & 26.62 & 5.78 & 5.80 & 37.26 & 37.27 & 30.14 & 30.17 \\
\hline 2004 & 1 & 25 & 26.58 & 26.63 & 5.77 & 5.80 & 37.26 & 37.26 & 30.16 & 30.18 \\
\hline 2004 & 1 & 26 & 26.55 & 26.57 & 5.76 & 5.77 & 37.25 & 37.26 & 30.14 & 30.15 \\
\hline 2004 & 1 & 27 & 26.51 & 26.57 & 5.74 & 5.77 & 37.25 & 37.25 & 30.11 & 30.13 \\
\hline 2004 & 1 & 28 & 26.56 & 26.58 & 5.77 & 5.78 & 37.25 & 37.25 & 30.15 & 30.16 \\
\hline 2004 & 1 & 29 & 26.56 & 26.58 & 5.76 & 5.77 & 37.24 & 37.25 & 30.16 & 30.17 \\
\hline 2004 & 1 & 30 & 26.54 & 26.56 & 5.75 & 5.77 & 37.24 & 37.24 & 30.15 & 30.17 \\
\hline 2004 & 1 & 31 & 26.58 & 26.62 & 5.77 & 5.80 & 37.24 & 37.24 & 30.18 & 30.21 \\
\hline 2004 & 2 & 1 & 26.60 & 26.62 & 5.78 & 5.80 & 37.24 & 37.24 & 30.22 & 30.22 \\
\hline 2004 & 2 & 2 & 26.55 & 26.61 & 5.75 & 5.78 & 37.23 & 37.24 & 30.19 & 30.22 \\
\hline 2004 & 2 & 3 & 26.52 & 26.59 & 5.74 & 5.79 & 37.23 & 37.24 & 30.16 & 30.20 \\
\hline 2004 & 2 & 4 & 26.60 & 26.63 & 5.78 & 5.80 & 37.23 & 37.24 & 30.22 & 30.23 \\
\hline 2004 & 2 & 5 & 26.56 & 26.63 & 5.75 & 5.79 & 37.23 & 37.24 & 30.21 & 30.24 \\
\hline 2004 & 2 & 6 & 26.49 & 26.52 & 5.72 & 5.74 & 37.23 & 37.23 & 30.14 & 30.17 \\
\hline 2004 & 2 & 7 & 26.55 & 26.59 & 5.76 & 5.79 & 37.23 & 37.23 & 30.17 & 30.21 \\
\hline 2004 & 2 & 8 & 26.58 & 26.61 & 5.77 & 5.80 & 37.23 & 37.23 & 30.22 & 30.23 \\
\hline 2004 & 2 & 9 & 26.53 & 26.56 & 5.74 & 5.76 & 37.23 & 37.23 & 30.18 & 30.20 \\
\hline 2004 & 2 & 10 & 26.53 & 26.57 & 5.74 & 5.77 & 37.23 & 37.23 & 30.18 & 30.21 \\
\hline 2004 & 2 & 11 & 26.55 & 26.56 & 5.76 & 5.77 & 37.23 & 37.23 & 30.21 & 30.21 \\
\hline 2004 & 2 & 12 & 26.54 & 26.56 & 5.75 & 5.77 & 37.22 & 37.23 & 30.21 & 30.22 \\
\hline 2004 & 2 & 13 & 26.53 & 26.55 & 5.73 & 5.75 & 37.23 & 37.24 & 30.20 & 30.21 \\
\hline 2004 & 2 & 14 & 26.54 & 26.58 & 5.74 & 5.77 & 37.24 & 37.24 & 30.20 & 30.23 \\
\hline 2004 & 2 & 15 & 26.58 & 26.61 & 5.77 & 5.79 & 37.24 & 37.24 & 30.24 & 30.25 \\
\hline 2004 & 2 & 16 & 26.59 & 26.60 & 5.77 & 5.79 & 37.24 & 37.24 & 30.26 & 30.26 \\
\hline 2004 & 2 & 17 & 26.56 & 26.58 & 5.76 & 5.78 & 37.24 & 37.24 & 30.25 & 30.25 \\
\hline 2004 & 2 & 18 & 26.53 & 26.58 & 5.75 & 5.78 & 37.24 & 37.24 & 30.22 & 30.25 \\
\hline 2004 & 2 & 19 & 26.52 & 26.54 & 5.74 & 5.76 & 37.24 & 37.24 & 30.20 & 30.21 \\
\hline 2004 & 2 & 20 & 26.47 & 26.54 & 5.71 & 5.75 & 37.24 & 37.24 & 30.15 & 30.20 \\
\hline 2004 & 2 & 21 & 26.51 & 26.54 & 5.73 & 5.76 & 37.24 & 37.25 & 30.16 & 30.19 \\
\hline 2004 & 2 & 22 & 26.54 & 26.56 & 5.74 & 5.76 & 37.25 & 37.25 & 30.18 & 30.19 \\
\hline 2004 & 2 & 23 & 26.50 & 26.54 & 5.71 & 5.74 & 37.25 & 37.25 & 30.15 & 30.17 \\
\hline 2004 & 2 & 24 & 26.50 & 26.54 & 5.70 & 5.72 & 37.25 & 37.25 & 30.14 & 30.15 \\
\hline 2004 & 2 & 25 & 26.49 & 26.51 & 5.71 & 5.72 & 37.25 & 37.25 & 30.14 & 30.15 \\
\hline 2004 & 2 & 26 & 26.47 & 26.49 & 5.69 & 5.71 & 37.25 & 37.25 & 30.12 & 30.13 \\
\hline 2004 & 2 & 27 & 26.46 & 26.49 & 5.69 & 5.70 & 37.25 & 37.25 & 30.11 & 30.13 \\
\hline 2004 & 2 & 28 & 26.44 & 26.47 & 5.68 & 5.68 & 37.26 & 37.26 & 30.10 & 30.11 \\
\hline 2004 & 2 & 29 & 26.41 & 26.44 & 5.66 & 5.67 & 37.26 & 37.26 & 30.06 & 30.07 \\
\hline 2004 & 3 & 1 & 26.39 & 26.42 & 5.64 & 5.67 & 37.26 & 37.26 & 30.00 & 30.03 \\
\hline 2004 & 3 & 2 & 26.38 & 26.41 & 5.62 & 5.64 & 37.26 & 37.26 & 29.95 & 29.96 \\
\hline 2004 & 3 & 3 & 26.36 & 26.40 & 5.60 & 5.63 & 37.26 & 37.26 & 29.92 & 29.94 \\
\hline 2004 & 3 & 4 & 26.33 & 26.35 & 5.57 & 5.59 & 37.25 & 37.25 & 29.87 & 29.89 \\
\hline 2004 & 3 & 5 & 26.19 & 26.28 & 5.51 & 5.54 & 37.24 & 37.25 & 29.77 & 29.83 \\
\hline 2004 & 3 & 6 & 26.25 & 26.29 & 5.52 & 5.53 & 37.24 & 37.24 & 29.72 & 29.74 \\
\hline 2004 & 3 & 7 & 26.22 & 26.27 & 5.48 & 5.53 & 37.24 & 37.24 & 29.67 & 29.71 \\
\hline 2004 & 3 & 8 & 26.26 & 26.28 & 5.48 & 5.50 & 37.23 & 37.24 & 29.65 & 29.66 \\
\hline
\end{tabular}


Table 7. Daily mean and maximum depth, in feet, of water below land surface in four monitoring wells in Oakland County, Michigan, June 2001 through March 2004 - continued $[--$, no data $]$

\begin{tabular}{|c|c|c|c|c|c|c|c|c|c|c|}
\hline \multirow[b]{2}{*}{ Year } & \multirow[b]{2}{*}{ Month } & \multirow[b]{2}{*}{ Day } & \multicolumn{2}{|c|}{$\begin{array}{c}\text { Holly State Rec. Area } \\
425116083321501\end{array}$} & \multicolumn{2}{|c|}{$\begin{array}{c}\text { Proud Lake State Rec. Area } \\
423423083324001\end{array}$} & \multicolumn{2}{|c|}{$\begin{array}{l}\text { Fish Lake Road } \\
424109083384301\end{array}$} & \multicolumn{2}{|c|}{$\begin{array}{l}\text { Teggerdine Road } \\
424133083293101\end{array}$} \\
\hline & & & Mean & Maximum & Mean & Maximum & Mean & Maximum & Mean & Maximum \\
\hline 2004 & 3 & 9 & 26.26 & 26.29 & 5.46 & 5.48 & 37.23 & 37.23 & 29.64 & 29.65 \\
\hline 2004 & 3 & 10 & 26.24 & 26.27 & 5.45 & 5.47 & 37.22 & 37.22 & 29.63 & 29.64 \\
\hline 2004 & 3 & 11 & 26.19 & 26.24 & 5.41 & 5.43 & 37.21 & 37.22 & 29.59 & 29.61 \\
\hline 2004 & 3 & 12 & 26.25 & 26.29 & 5.42 & 5.44 & 37.21 & 37.21 & 29.60 & 29.62 \\
\hline 2004 & 3 & 13 & 26.27 & 26.32 & 5.43 & 5.45 & 37.20 & 37.21 & 29.64 & 29.65 \\
\hline 2004 & 3 & 14 & 26.21 & 26.25 & 5.39 & 5.41 & 37.20 & 37.21 & 29.59 & 29.61 \\
\hline 2004 & 3 & 15 & 26.27 & 26.30 & 5.41 & 5.43 & 37.20 & 37.21 & 29.63 & 29.65 \\
\hline 2004 & 3 & 16 & 26.24 & 26.26 & 5.38 & 5.40 & 37.19 & 37.19 & 29.61 & 29.63 \\
\hline 2004 & 3 & 17 & 26.24 & 26.27 & 5.37 & 5.38 & 37.19 & 37.19 & 29.61 & 29.62 \\
\hline 2004 & 3 & 18 & 26.25 & 26.28 & 5.37 & 5.40 & 37.18 & 37.19 & 29.63 & 29.65 \\
\hline 2004 & 3 & 19 & 26.28 & 26.32 & 5.38 & 5.40 & 37.18 & 37.19 & 29.67 & 29.69 \\
\hline 2004 & 3 & 20 & 26.18 & 26.24 & 5.32 & 5.37 & 37.18 & 37.19 & 29.60 & 29.65 \\
\hline 2004 & 3 & 21 & 26.22 & 26.24 & 5.34 & 5.36 & 37.17 & 37.18 & 29.61 & 29.62 \\
\hline 2004 & 3 & 22 & 26.22 & 26.26 & 5.34 & 5.36 & 37.17 & 37.17 & 29.61 & 29.63 \\
\hline 2004 & 3 & 23 & 26.21 & 26.24 & 5.32 & 5.34 & 37.17 & 37.17 & 29.60 & 29.61 \\
\hline 2004 & 3 & 24 & 26.20 & 26.24 & 5.31 & 5.33 & 37.16 & 37.16 & 29.59 & 29.60 \\
\hline 2004 & 3 & 25 & 26.19 & 26.21 & 5.28 & 5.30 & 37.16 & 37.16 & 29.58 & 29.59 \\
\hline 2004 & 3 & 26 & 26.14 & 26.21 & 5.26 & 5.27 & 37.14 & 37.15 & 29.55 & 29.56 \\
\hline 2004 & 3 & 27 & 26.13 & 26.15 & 5.26 & 5.27 & 37.14 & 37.14 & 29.53 & 29.54 \\
\hline 2004 & 3 & 28 & 26.11 & 26.14 & 5.24 & 5.26 & 37.13 & 37.14 & 29.51 & 29.52 \\
\hline 2004 & 3 & 29 & 26.11 & 26.13 & 5.24 & 5.25 & 37.13 & 37.13 & 29.50 & 29.50 \\
\hline 2004 & 3 & 30 & 26.11 & 26.13 & 5.22 & 5.24 & 37.12 & 37.13 & 29.49 & 29.50 \\
\hline 2004 & 3 & 31 & 26.11 & 26.14 & 5.22 & 5.23 & 37.12 & 37.12 & 29.48 & 29.49 \\
\hline
\end{tabular}


Table 8. Measured depth, in feet, to water in monitoring well 424133083293201 Oakland County, Michigan.

\begin{tabular}{rrr}
\multicolumn{1}{c}{ Date } & Time & Depth \\
\hline April 9, 2001 & 1530 & 8.40 \\
June 13, 2001 & 1715 & 8.55 \\
June 22, 2001 & 1315 & 9.36 \\
July 7, 2001 & 1145 & 10.16 \\
July 13, 2001 & 1334 & 10.45 \\
July 25, 2001 & 915 & 10.90 \\
August 17, 2001 & 1230 & 11.41 \\
September 4, 2001 & 1320 & 11.06 \\
October 23, 2001 & 940 & 9.16 \\
January 4, 2002 & 1527 & 9.21 \\
April 25, 2002 & 1515 & 8.59 \\
June 21, 2002 & 1330 & 9.73 \\
July 30, 2002 & 1103 & 11.06 \\
September 6, 2002 & 1403 & 11.64 \\
September 19, 2002 & 927 & 11.89 \\
October 31, 2002 & 1315 & 11.33 \\
December 6, 2002 & 1100 & 10.63 \\
January 13, 2003 & 1100 & 10.57 \\
February 21, 2003 & 1600 & 10.55 \\
March 25, 2003 & 1730 & 10.05 \\
April 21, 2003 & 1400 & 9.53 \\
June 2, 2003 & 830 & 10.30 \\
June 26, 2003 & 1500 & 10.98 \\
August 15, 2003 & 1050 & 11.65 \\
September 8, 2003 & 915 & 12.36 \\
October 8, 2003 & 1000 & 11.75 \\
November 18, 2003 & 1510 & 10.51 \\
December 15, 2003 & 945 & 10.27 \\
\hline & &
\end{tabular}


Table 9.Results of synoptic stream-water-quality sample analysis in and around Oakland County, Michigan between September 2001 and September 2003

$[\mathrm{mg} / \mathrm{L}$, milligrams per liter; $\mathrm{mg} / \mathrm{L}$, micrograms per liter; $\mathrm{mS} / \mathrm{cm}$, microsiemens per centimeter; wf, filtered water; $\mathrm{mL}$, milliliter]

\begin{tabular}{|c|c|c|c|c|c|c|c|c|}
\hline 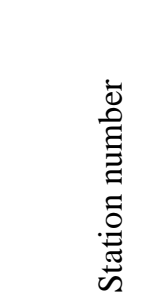 & 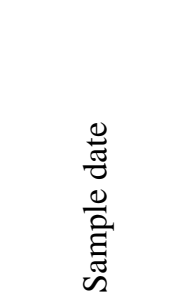 & 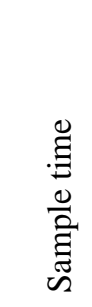 & 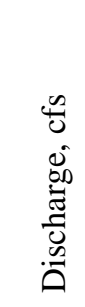 & 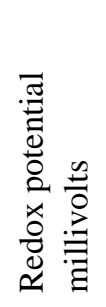 & 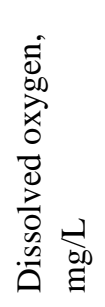 & 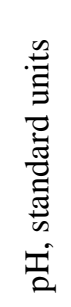 & 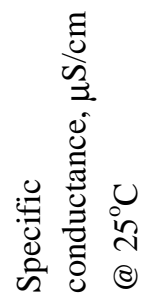 & 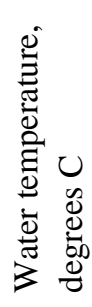 \\
\hline 04143830 & $09-17-2001$ & 1415 & 14 & -- & 9.7 & 7.8 & 544 & 19.1 \\
\hline 04143830 & $09-19-2001$ & 1445 & 32 & -- & 7.8 & 7.7 & 500 & 18.6 \\
\hline 04143830 & $11-14-2001$ & 930 & -- & -- & 12.2 & 7.7 & 567 & 7.4 \\
\hline 04143830 & $01-24-2002$ & 1500 & 42 & -- & 13.9 & 7.6 & 604 & 1.1 \\
\hline 04143830 & $03-11-2002$ & 1545 & 116 & -- & 14.6 & 7.5 & 535 & 1.8 \\
\hline 04143830 & 04-29-2002 & 1345 & -- & -- & -- & 8.3 & 522 & -- \\
\hline 04143830 & 06-06-2002 & 1430 & -- & -- & 8.3 & 7.8 & 454 & 18.8 \\
\hline 04143830 & 07-10-2002 & 858 & 14 & -- & 5.5 & 8.0 & 478 & 23.0 \\
\hline 04143830 & 08-21-2002 & 1545 & 21 & 166 & 8.8 & 8.0 & 522 & 24.8 \\
\hline 04143830 & $11-11-2002$ & 900 & 25 & 166 & 9.6 & 7.8 & 542 & 9.7 \\
\hline 04143830 & $12-11-2002$ & 1600 & 15 & -- & -- & 8.0 & 651 & 0.6 \\
\hline 04143830 & $01-28-2003$ & 930 & 20 & -- & 12 & 7.7 & 680 & -0.2 \\
\hline 04143830 & $04-22-2003$ & 900 & 41 & -- & 8.4 & 7.7 & 539 & 10.0 \\
\hline 04143830 & $05-20-2003$ & 1000 & 40 & -- & 7.2 & 8.0 & 526 & 19.1 \\
\hline 04143830 & $06-12-2003$ & 1430 & 44 & -- & 8.1 & 7.6 & 481 & 17.7 \\
\hline 04143830 & $07-23-2003$ & 845 & 7.2 & 347 & 5.8 & 7.4 & 542 & 20.5 \\
\hline 04143830 & $08-05-2003$ & 1845 & 9.7 & 522 & 7.4 & 8.1 & 590 & 25.8 \\
\hline 04143830 & 09-09-2003 & 900 & 4.5 & 382 & 6.2 & 7.6 & 571 & 19.6 \\
\hline 04148035 & 07-10-2002 & 1042 & 5.1 & -- & 8.2 & 7.8 & 776 & 17.0 \\
\hline 04148035 & $08-21-2002$ & 1430 & 6.8 & 182 & 8.8 & 7.8 & 755 & 17.9 \\
\hline 04148035 & $12-11-2002$ & 1445 & 6.8 & -- & -- & 8.0 & 802 & 0.6 \\
\hline 04148035 & $04-22-2003$ & 1030 & 38 & 323 & 9.2 & 7.8 & 639 & 8.5 \\
\hline 04148035 & $06-12-2003$ & 1600 & 32 & -- & 8.2 & 7.5 & 594 & 15.6 \\
\hline 04148035 & $07-23-2003$ & 1715 & 6.2 & 355 & 7.9 & 7.9 & 747 & 18.4 \\
\hline 04148035 & 08-05-2003 & 1745 & 6.1 & 551 & 8.4 & 8.1 & 784 & 20.4 \\
\hline 04148035 & 09-09-2003 & 1000 & 4.2 & 332 & 8.4 & 8.0 & 806 & 14.8 \\
\hline 04160800 & 09-17-2001 & 830 & 1.6 & -- & 7.8 & 7.6 & 712 & 12.8 \\
\hline 04160800 & 04-29-2002 & 930 & -- & -- & -- & 8.1 & 655 & -- \\
\hline 04160800 & 06-04-2002 & 945 & 31 & -- & 11.5 & 7.6 & 515 & 13.5 \\
\hline 04160800 & 07-08-2002 & 1046 & 3.8 & -- & -- & 8.2 & 610 & -- \\
\hline 04160800 & 08-20-2002 & 850 & 1.3 & -- & -- & 8.1 & 707 & -- \\
\hline 04160800 & $12-12-2002$ & 1000 & 1.8 & -- & 8.9 & 7.2 & 781 & -0.1 \\
\hline 04160800 & 02-02-2003 & 1700 & 3.2 & -- & 11.8 & 7.5 & 729 & -0.2 \\
\hline 04160800 & $04-22-2003$ & 1500 & 14 & 304 & 9.8 & 7.7 & 741 & 8.8 \\
\hline 04160800 & $05-20-2003$ & 1130 & 8.5 & -- & 7.4 & 7.8 & E720 & 17.1 \\
\hline 04160800 & $06-11-2003$ & 945 & 12 & -- & 8.2 & 7.4 & 754 & 17.4 \\
\hline 04160800 & $06-12-2003$ & 1715 & 20 & -- & 7.6 & 7.4 & 694 & 16.4 \\
\hline 04160800 & $07-23-2003$ & 1015 & 1.4 & 392 & 7.3 & 7.8 & 648 & 18.4 \\
\hline 04160800 & 08-05-2003 & 1930 & 2.2 & 519 & 6.6 & 8.0 & 661 & 23.5 \\
\hline 04160800 & 09-09-2003 & 1130 & 0.55 & -- & 8.3 & 8.1 & 675 & 18.5 \\
\hline
\end{tabular}


Table 9.Results of synoptic stream-water-quality sample analysis in and around Oakland County, Michigan between September 2001 and September 2003- continued

$[\mathrm{mg} / \mathrm{L}$, milligrams per liter; $\mathrm{mg} / \mathrm{L}$, micrograms per liter; $\mathrm{mS} / \mathrm{cm}$, microsiemens per centimeter; wf, filtered water; $\mathrm{mL}$, milliliter]

\begin{tabular}{|c|c|c|c|c|c|c|c|c|}
\hline 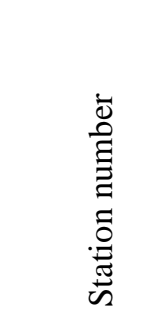 & 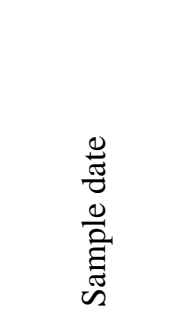 & 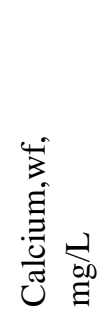 & 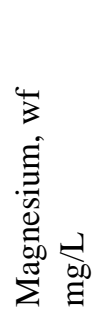 & 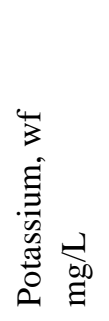 & 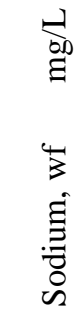 & 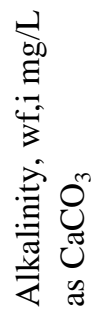 & 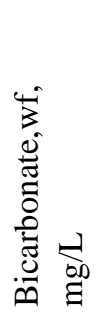 & 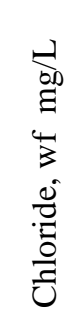 \\
\hline 04143830 & $09-17-2001$ & 48.2 & 24.7 & 2.6 & 26.5 & 192 & 234 & 54 \\
\hline 04143830 & 09-19-2001 & 42.9 & 22.5 & 3.0 & 21.7 & 156 & 190 & 44 \\
\hline 04143830 & $11-14-2001$ & 64.6 & 23.0 & 2.1 & 19.5 & 208 & 254 & 42 \\
\hline 04143830 & 01-24-2002 & 69.7 & 22.8 & 2.0 & 19.1 & 222 & 271 & 40 \\
\hline 04143830 & 03-11-2002 & 59.5 & 19.2 & 1.5 & 17 & -- & -- & 35 \\
\hline 04143830 & 04-29-2002 & 60.3 & 21.3 & 1.6 & 18.7 & 200 & -- & 39 \\
\hline 04143830 & 06-06-2002 & 51.8 & 19.1 & 1.3 & 14.1 & 184 & 225 & 31 \\
\hline 04143830 & 07-10-2002 & 37.7 & 22.5 & 2.0 & 25.5 & 114 & 139 & 47 \\
\hline 04143830 & 08-21-2002 & 55.3 & 23.8 & 2.5 & 23.8 & 172 & 210 & 45 \\
\hline 04143830 & $11-11-2002$ & 60.5 & 24.8 & 2.0 & 19.5 & 222 & -- & 37 \\
\hline 04143830 & $12-11-2002$ & 73.3 & 26.6 & 2.9 & 24.8 & 240 & 293 & 47 \\
\hline 04143830 & $01-28-2003$ & 86.2 & 29.4 & 2.5 & 25.8 & 289 & -- & 44 \\
\hline 04143830 & $04-22-2003$ & 64.8 & 21.1 & 2.6 & 17.6 & 214 & 261 & 38 \\
\hline 04143830 & $05-20-2003$ & 60.4 & 21.9 & 2.1 & 19.8 & 188 & 229 & 40 \\
\hline 04143830 & $06-12-2003$ & 54.2 & 19.5 & 2.0 & 22.8 & 172 & 210 & 43 \\
\hline 04143830 & $07-23-2003$ & 49.0 & 23.3 & 3.2 & 31.7 & 184 & 224 & 59 \\
\hline 04143830 & 08-05-2003 & 48.8 & 25.2 & 3.4 & 36.7 & E168 & 205 & 67 \\
\hline 04143830 & 09-09-2003 & 46.2 & 25.6 & 4.2 & 32.5 & 196 & 239 & 66 \\
\hline 04148035 & 07-10-2002 & 83.7 & 27.9 & 1.6 & 39.9 & 264 & 322 & 78 \\
\hline 04148035 & $08-21-2002$ & 85.2 & 27.3 & 2.0 & 39.9 & 274 & 334 & 77 \\
\hline 04148035 & $12-11-2002$ & 90.1 & 28.6 & 2.1 & 40.1 & 266 & 324 & 79 \\
\hline 04148035 & $04-22-2003$ & 71.1 & 21.9 & 2.8 & 29.9 & 212 & 258 & 65 \\
\hline 04148035 & $06-12-2003$ & 71.1 & 21.5 & 1.9 & 27.7 & 204 & 245 & 55 \\
\hline 04148035 & 07-23-2003 & 84.1 & 25.9 & 1.9 & 43.6 & 264 & 322 & 82 \\
\hline 04148035 & 08-05-2003 & 85.8 & 26.7 & 2.1 & 45.5 & 262 & 320 & 85 \\
\hline 04148035 & 09-09-2003 & 85.7 & 27.6 & 2.0 & 45.4 & 270 & 330 & 90 \\
\hline 04160800 & 09-17-2001 & 80.5 & 22.4 & 2.1 & 32.9 & 230 & 281 & 75 \\
\hline 04160800 & 04-29-2002 & 68.1 & 17.7 & 1.9 & 42.3 & 496 & -- & 85 \\
\hline 04160800 & 06-04-2002 & 52.0 & 13.5 & 1.8 & 31.9 & 152 & 185 & 63 \\
\hline 04160800 & 07-08-2002 & 60.9 & 19.1 & 1.5 & 35.6 & 295 & 360 & 73 \\
\hline 04160800 & 08-20-2002 & 77.2 & 22.6 & 2.3 & 37.6 & 227 & 277 & 84 \\
\hline 04160800 & $12-12-2002$ & 92.0 & 24.1 & 2.2 & 35 & 244 & 298 & 82 \\
\hline 04160800 & $02-02-2003$ & 86.4 & 22.1 & 2.0 & 32.3 & 266 & 325 & 72 \\
\hline 04160800 & $04-22-2003$ & 70.2 & 17.4 & 2.7 & 56.8 & 164 & 201 & 112 \\
\hline 04160800 & $05-20-2003$ & 73.2 & 18.5 & 2.2 & 47.9 & 178 & 217 & 99 \\
\hline 04160800 & 06-11-2003 & 73.3 & 17.8 & 1.9 & 57.4 & 176 & 215 & 110 \\
\hline 04160800 & $06-12-2003$ & 70.1 & 17.2 & 2.0 & 50.7 & 180 & 220 & 101 \\
\hline 04160800 & $07-23-2003$ & 74.3 & 21.1 & 1.9 & 36.1 & 200 & 244 & 81 \\
\hline 04160800 & 08-05-2003 & 72.9 & 20.8 & 2.1 & 36.5 & 212 & 259 & 81 \\
\hline 04160800 & 09-09-2003 & 74.1 & 22.9 & 2.3 & 34.8 & 212 & 258 & 81 \\
\hline
\end{tabular}


Table 9.Results of synoptic stream-water-quality sample analysis in and around Oakland County, Michigan between September 2001 and September 2003- continued

[mg/L, milligrams per liter; $\mathrm{mg} / \mathrm{L}$, micrograms per liter; $\mathrm{mS} / \mathrm{cm}$, microsiemens per centimeter; wf, filtered water; $\mathrm{mL}$, milliliter]

\begin{tabular}{|c|c|c|c|c|c|c|c|c|c|}
\hline 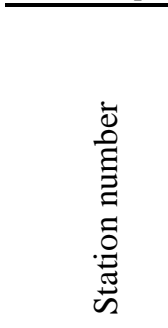 & 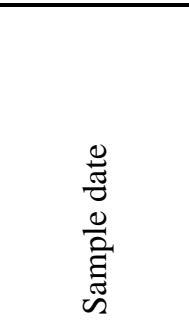 & 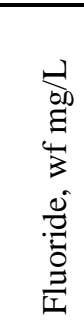 & 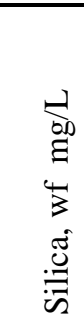 & 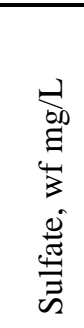 & 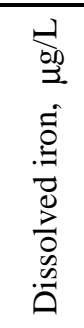 & 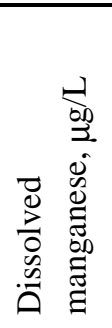 & 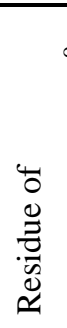 & 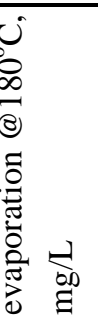 & 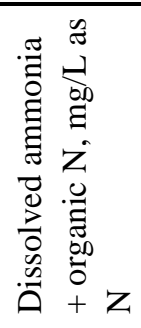 \\
\hline 04143830 & 09-17-2001 & 0.2 & 13.2 & 18.0 & 45 & 16.7 & & 318 & 0.44 \\
\hline 04143830 & $09-19-2001$ & 0.2 & 11.9 & 16.5 & 31 & 45.7 & & 289 & 0.5 \\
\hline 04143830 & $11-14-2001$ & 0.2 & 9.6 & 24.7 & 30 & 15.2 & & 334 & 0.33 \\
\hline 04143830 & 01-24-2002 & 0.2 & 9.6 & 22.1 & 30 & 23.4 & & 362 & 0.28 \\
\hline 04143830 & $03-11-2002$ & 0.2 & 7.5 & 19.1 & 22 & 8.3 & & 298 & 0.27 \\
\hline 04143830 & $04-29-2002$ & 0.2 & 4.7 & 16.2 & 70 & 29.3 & & 310 & 0.37 \\
\hline 04143830 & 06-06-2002 & 0.2 & 7.1 & 12.7 & 52 & 14.6 & & 301 & 0.46 \\
\hline 04143830 & $07-10-2002$ & 0.2 & 19.1 & 13.1 & 59 & 19.9 & & 286 & 0.55 \\
\hline 04143830 & 08-21-2002 & 0.2 & 18.1 & 16.8 & 48 & 10.3 & & 350 & 0.61 \\
\hline 04143830 & $11-11-2002$ & 0.2 & 11.1 & 21.8 & 31 & 20.8 & & 322 & 0.3 \\
\hline 04143830 & $12-11-2002$ & 0.2 & 12.1 & 24.2 & 42 & 15.7 & & 401 & 0.45 \\
\hline 04143830 & $01-28-2003$ & 0.2 & 14.0 & 25.8 & 33 & 21.1 & & 413 & 0.36 \\
\hline 04143830 & $04-22-2003$ & 0.2 & 5.5 & 21.9 & 79 & 82.7 & & 319 & 0.46 \\
\hline 04143830 & $05-20-2003$ & 0.2 & 6.3 & 19.1 & 101 & 47.8 & & 325 & 0.56 \\
\hline 04143830 & $06-12-2003$ & 0.2 & 7.0 & 14.9 & 90 & 51.1 & & 290 & 0.54 \\
\hline 04143830 & $07-23-2003$ & 0.3 & 16.5 & 16.2 & 46 & 40.2 & & 342 & 0.66 \\
\hline 04143830 & 08-05-2003 & 0.3 & 18.4 & 17.0 & 47 & 25.3 & & 382 & 0.8 \\
\hline 04143830 & 09-09-2003 & 0.3 & 15.2 & 20.8 & 56 & 34.6 & & 343 & 0.55 \\
\hline 04148035 & 07-10-2002 & 0.2 & 14.5 & 26.8 & 66 & 66.8 & & 458 & 0.29 \\
\hline 04148035 & 08-21-2002 & 0.2 & 15.4 & 27.1 & 52 & 68.2 & & 505 & 0.26 \\
\hline 04148035 & $12-11-2002$ & 0.2 & 12.0 & 34.7 & 39 & 65.7 & & 497 & 0.24 \\
\hline 04148035 & $04-22-2003$ & 0.2 & 5.5 & 33.2 & 103 & 47.4 & & 388 & 0.53 \\
\hline 04148035 & $06-12-2003$ & 0.2 & 9.2 & 24.7 & 121 & 78.9 & & 370 & 0.57 \\
\hline 04148035 & 07-23-2003 & 0.3 & 14.4 & 27.2 & 55 & 57.3 & & 474 & 0.34 \\
\hline 04148035 & 08-05-2003 & 0.3 & 13.8 & 28.3 & 41 & 44.7 & & 516 & 0.28 \\
\hline 04148035 & 09-09-2003 & 0.3 & 15.0 & 29.4 & 47 & 53.8 & & 493 & 0.23 \\
\hline 04160800 & 09-17-2001 & E. 2 & 8.7 & 30.7 & 88 & 89.1 & & 413 & 0.44 \\
\hline 04160800 & 04-29-2002 & E.10 & 3.5 & 21.2 & 172 & 60.7 & & 392 & 0.48 \\
\hline 04160800 & 06-04-2002 & E.10 & 4.2 & 16.4 & 194 & 125 & & 304 & 0.64 \\
\hline 04160800 & 07-08-2002 & $<.10$ & 6.8 & 19.8 & 110 & 56.9 & & 378 & 0.53 \\
\hline 04160800 & 08-20-2002 & 0.2 & 10.5 & 30.0 & 120 & 70.8 & & 447 & 0.38 \\
\hline 04160800 & $12-12-2002$ & $<.17$ & 10.4 & 44.8 & 86 & 101 & & 471 & 0.46 \\
\hline 04160800 & $02-02-2003$ & 0.1 & 9.8 & 36.4 & 97 & 164 & & 426 & 0.52 \\
\hline 04160800 & $04-22-2003$ & 0.1 & 5.1 & 46.8 & 142 & 50 & & 459 & 0.61 \\
\hline 04160800 & $05-20-2003$ & $<.2$ & 4.0 & 33.3 & 278 & 100 & & 454 & 0.66 \\
\hline 04160800 & 06-11-2003 & $<.2$ & 4.8 & 29.5 & 220 & 53.5 & & 482 & 0.83 \\
\hline 04160800 & $06-12-2003$ & $<.2$ & 5.9 & 27.4 & 247 & 54.4 & & 424 & 0.74 \\
\hline 04160800 & 07-23-2003 & 0.2 & 9.5 & 29.1 & 110 & 83.8 & & 443 & 0.58 \\
\hline 04160800 & 08-05-2003 & $<.2$ & 9.4 & 30.5 & 69 & 53.1 & & 482 & 0.6 \\
\hline 04160800 & 09-09-2003 & $<.2$ & 7.4 & 33.2 & 68 & 50.8 & & 415 & 0.47 \\
\hline
\end{tabular}


Table 9.Results of synoptic stream-water-quality sample analysis in and around Oakland County, Michigan between September 2001 and September 2003- continued

$[\mathrm{mg} / \mathrm{L}$, milligrams per liter; $\mathrm{mg} / \mathrm{L}$, micrograms per liter; $\mathrm{mS} / \mathrm{cm}$, microsiemens per centimeter; wf, filtered water; $\mathrm{mL}$, milliliter]

\begin{tabular}{|c|c|c|c|c|c|c|c|c|}
\hline 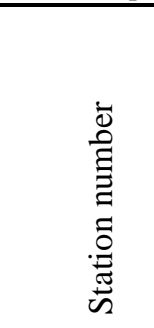 & 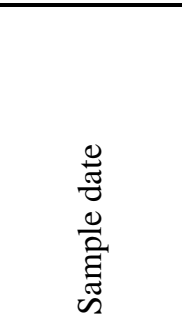 & 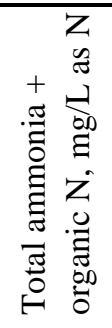 & 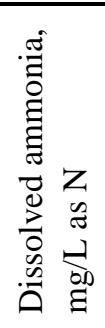 & 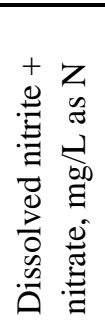 & 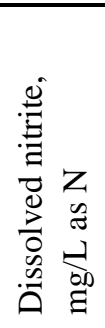 & 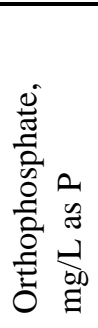 & 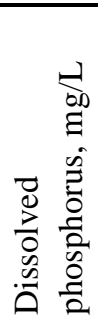 & 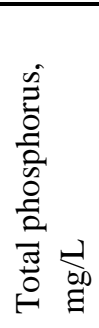 \\
\hline 04143830 & 09-17-2001 & 0.55 & $<<.04$ & 0.46 & $<.006$ & 0.02 & 0.035 & 0.031 \\
\hline 04143830 & 09-19-2001 & 1 & 0.04 & 0.67 & 0.009 & E.01 & 0.027 & 0.044 \\
\hline 04143830 & $11-14-2001$ & 0.41 & $<.04$ & 0.24 & $<.008$ & $<.02$ & 0.007 & E.003 \\
\hline 04143830 & 01-24-2002 & 0.37 & $<.04$ & 0.42 & $<.008$ & E.01 & 0.024 & 0.01 \\
\hline 04143830 & 03-11-2002 & 0.6 & $<.04$ & 0.2 & $<.008$ & $<.02$ & 0.005 & $<.004$ \\
\hline 04143830 & $04-29-2002$ & 0.52 & $<.04$ & 0.12 & E.005 & E.01 & 0.009 & E.04 \\
\hline 04143830 & 06-06-2002 & 0.57 & 0.06 & 0.21 & E.004 & $<.02$ & 0.013 & $<.004$ \\
\hline 04143830 & $07-10-2002$ & 0.78 & E.03 & 0.53 & 0.009 & 0.02 & 0.026 & E.05 \\
\hline 04143830 & 08-21-2002 & 0.64 & 0.08 & 0.71 & 0.015 & E.01 & 0.021 & 0.031 \\
\hline 04143830 & $11-11-2002$ & 0.37 & $<.04$ & 0.18 & E.004 & $<.02$ & 0.01 & 0.022 \\
\hline 04143830 & $12-11-2002$ & 0.59 & 0.12 & 0.96 & 0.01 & $<.02$ & 0.012 & 0.026 \\
\hline 04143830 & 01-28-2003 & 0.41 & 0.04 & 0.57 & E.004 & $<.02$ & 0.008 & 0.02 \\
\hline 04143830 & $04-22-2003$ & 0.58 & E.03 & 0.18 & $<.008$ & $<.02$ & 0.01 & 0.022 \\
\hline 04143830 & $05-20-2003$ & 0.6 & E.03 & 0.33 & E.007 & $<.02$ & 0.013 & 0.027 \\
\hline 04143830 & $06-12-2003$ & 0.7 & 0.04 & 0.46 & 0.009 & $<.02$ & 0.017 & 0.049 \\
\hline 04143830 & $07-23-2003$ & 0.64 & 0.09 & 0.88 & 0.012 & E.02 & 0.033 & 0.046 \\
\hline 04143830 & 08-05-2003 & 0.82 & $<.04$ & 1.99 & 0.029 & E.01 & 0.028 & 0.036 \\
\hline 04143830 & 09-09-2003 & 0.68 & E.03 & 1.03 & 0.009 & E.02 & 0.028 & 0.041 \\
\hline 04148035 & 07-10-2002 & 0.46 & $<.04$ & 0.9 & 0.009 & E.02 & 0.023 & E.06 \\
\hline 04148035 & $08-21-2002$ & 0.34 & E.02 & 0.58 & E.005 & 0.02 & 0.025 & 0.046 \\
\hline 04148035 & $12-11-2002$ & 0.36 & 0.05 & 0.64 & 0.008 & $<.02$ & 0.007 & 0.021 \\
\hline 04148035 & 04-22-2003 & 0.6 & 0.05 & 0.21 & E.005 & $<.02$ & 0.012 & 0.03 \\
\hline 04148035 & $06-12-2003$ & 1.4 & E.04 & 0.23 & E.007 & E.01 & 0.025 & 0.125 \\
\hline 04148035 & $07-23-2003$ & 0.42 & 0.04 & 0.57 & E.005 & E.01 & 0.019 & 0.039 \\
\hline 04148035 & 08-05-2003 & 0.33 & $<.04$ & 0.42 & E.004 & E.01 & 0.018 & 0.03 \\
\hline 04148035 & 09-09-2003 & 0.29 & E.03 & 0.42 & $<.008$ & E.01 & 0.015 & 0.025 \\
\hline 04160800 & 09-17-2001 & 0.56 & E.03 & 0.11 & $<.006$ & $<.02$ & 0.008 & 0.01 \\
\hline 04160800 & 04-29-2002 & 0.5 & $<.04$ & E.04 & E.005 & $<.02$ & 0.006 & $<.06$ \\
\hline 04160800 & 06-04-2002 & 1.1 & 0.07 & 0.17 & 0.008 & $<.02$ & 0.013 & E.003 \\
\hline 04160800 & 07-08-2002 & 0.58 & E.03 & 0.23 & 0.01 & $<.02$ & 0.011 & E.03 \\
\hline 04160800 & 08-20-2002 & 0.71 & E.04 & 0.23 & E.004 & E.01 & 0.02 & 0.054 \\
\hline 04160800 & $12-12-2002$ & 0.54 & 0.18 & 0.11 & E.004 & $<.02$ & 0.004 & 0.013 \\
\hline 04160800 & 02-02-2003 & 0.58 & 0.25 & 0.15 & E.005 & $<.02$ & E.003 & 0.013 \\
\hline 04160800 & $04-22-2003$ & 0.72 & $<.04$ & 0.08 & $<.008$ & $<.02$ & 0.011 & 0.018 \\
\hline 04160800 & $05-20-2003$ & 0.7 & 0.04 & 0.08 & E.006 & E.01 & 0.01 & 0.021 \\
\hline 04160800 & $06-11-2003$ & 0.71 & E.04 & 0.11 & 0.024 & $<.02$ & 0.026 & 0.012 \\
\hline 04160800 & $06-12-2003$ & 0.88 & E.03 & 0.08 & E.005 & $<.02$ & 0.014 & 0.039 \\
\hline 04160800 & $07-23-2003$ & 0.69 & 0.04 & 0.15 & E.005 & $<.02$ & 0.017 & 0.036 \\
\hline 04160800 & 08-05-2003 & 0.7 & $<.04$ & 0.14 & E.004 & $<.02$ & 0.017 & 0.036 \\
\hline 04160800 & 09-09-2003 & 0.52 & $<.04$ & $<.06$ & $<.008$ & $<.02$ & 0.009 & 0.021 \\
\hline
\end{tabular}


Table 9.Results of synoptic stream-water-quality sample analysis in and around Oakland County, Michigan between September 2001 and September 2003- continued

$[\mathrm{mg} / \mathrm{L}$, milligrams per liter; $\mathrm{mg} / \mathrm{L}$, micrograms per liter; $\mathrm{mS} / \mathrm{cm}$, microsiemens per centimeter; wf, filtered water; $\mathrm{mL}$, milliliter]
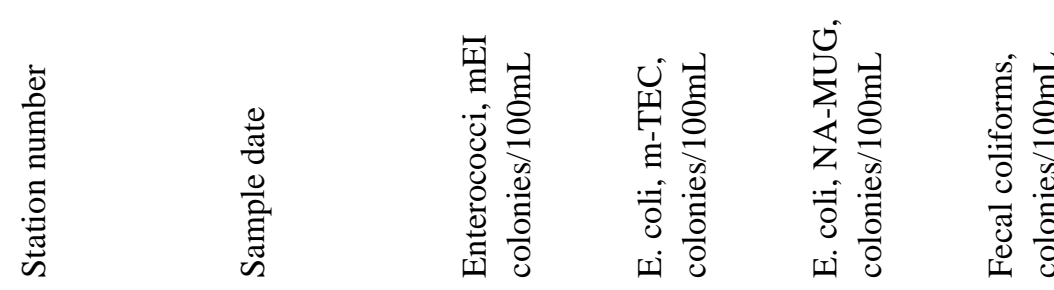

\begin{tabular}{|c|c|c|c|c|c|}
\hline 04143830 & $09-17-2001$ & -- & -- & -- & -- \\
\hline 04143830 & 09-19-2001 & -- & -- & -- & -- \\
\hline 04143830 & $11-14-2001$ & -- & -- & -- & -- \\
\hline 04143830 & 01-24-2002 & -- & -- & -- & -- \\
\hline 04143830 & 03-11-2002 & -- & 18 & -- & -- \\
\hline 04143830 & 04-29-2002 & -- & 10 & -- & -- \\
\hline 04143830 & 06-06-2002 & -- & -- & -- & -- \\
\hline 04143830 & $07-10-2002$ & -- & -- & -- & -- \\
\hline 04143830 & $08-21-2002$ & -- & 120 & -- & -- \\
\hline 04143830 & $11-11-2002$ & -- & 59 & -- & -- \\
\hline 04143830 & $12-11-2002$ & -- & -- & -- & -- \\
\hline 04143830 & $01-28-2003$ & -- & -- & -- & -- \\
\hline 04143830 & $04-22-2003$ & -- & 30 & -- & -- \\
\hline 04143830 & $05-20-2003$ & -- & 140 & -- & -- \\
\hline 04143830 & $06-12-2003$ & -- & 130 & -- & -- \\
\hline 04143830 & $07-23-2003$ & -- & -- & -- & -- \\
\hline 04143830 & $08-05-2003$ & E200 & -- & 140 & E980 \\
\hline 04143830 & 09-09-2003 & E390 & -- & E100 & E290 \\
\hline 04148035 & 07-10-2002 & -- & -- & -- & - \\
\hline 04148035 & $08-21-2002$ & -- & 340 & -- & -- \\
\hline 04148035 & $12-11-2002$ & -- & -- & -- & -- \\
\hline 04148035 & $04-22-2003$ & -- & 200 & -- & -- \\
\hline 04148035 & $06-12-2003$ & -- & -- & -- & -- \\
\hline 04148035 & $07-23-2003$ & -- & -- & -- & -- \\
\hline 04148035 & 08-05-2003 & E400 & -- & E300 & E2400 \\
\hline 04148035 & 09-09-2003 & E650 & -- & E500 & E600 \\
\hline 04160800 & 09-17-2001 & -- & -- & -- & -- \\
\hline 04160800 & 04-29-2002 & -- & 46 & -- & -- \\
\hline 04160800 & 06-04-2002 & -- & 9500 & -- & -- \\
\hline 04160800 & 07-08-2002 & -- & -- & -- & -- \\
\hline 04160800 & $08-20-2002$ & -- & 770 & -- & -- \\
\hline 04160800 & $12-12-2002$ & -- & -- & -- & -- \\
\hline 04160800 & 02-02-2003 & -- & 8 & -- & -- \\
\hline 04160800 & $04-22-2003$ & -- & 200 & -- & -- \\
\hline 04160800 & $05-20-2003$ & -- & 700 & -- & -- \\
\hline 04160800 & 06-11-2003 & -- & 70 & -- & -- \\
\hline 04160800 & $06-12-2003$ & -- & 80 & -- & -- \\
\hline 04160800 & $07-23-2003$ & -- & -- & -- & -- \\
\hline 04160800 & 08-05-2003 & 96 & -- & 120 & $\mathrm{E} 430$ \\
\hline 04160800 & 09-09-2003 & $\mathrm{E} 300$ & -- & E220 & E1040 \\
\hline
\end{tabular}


Table 9.Results of synoptic stream-water-quality sample analysis in and around Oakland County, Michigan between September 2001 and September 2003- continued

[mg/L, milligrams per liter; $\mathrm{mg} / \mathrm{L}$, micrograms per liter; $\mathrm{mS} / \mathrm{cm}$, microsiemens per centimeter; wf, filtered water; $\mathrm{mL}$, milliliter]

\begin{tabular}{|c|c|c|c|c|c|c|c|c|}
\hline 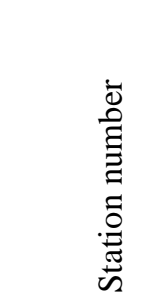 & 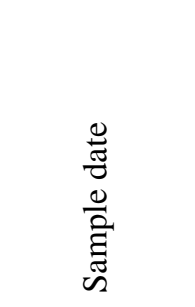 & 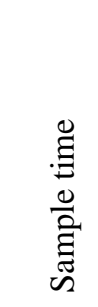 & 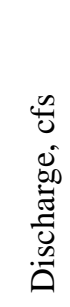 & 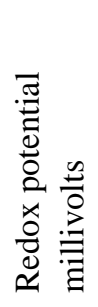 & 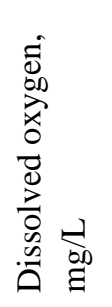 & 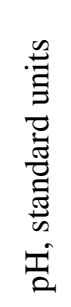 & 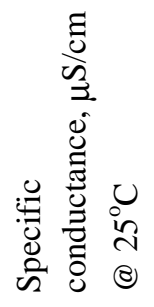 & 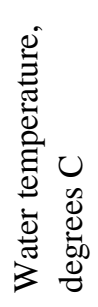 \\
\hline 04160900 & $09-17-2001$ & 1030 & 1.4 & -- & 5.5 & 7.0 & 879 & 13.5 \\
\hline 04160900 & $11-14-2001$ & 1300 & 66 & -- & 10.5 & 7.7 & 720 & 8.9 \\
\hline 04160900 & $01-22-2002$ & 915 & 57 & -- & 12.2 & 7.0 & 739 & 0.7 \\
\hline 04160900 & 03-11-2002 & 915 & 106 & -- & 13 & 7.6 & 747 & 1.8 \\
\hline 04160900 & 04-29-2002 & 800 & 84 & -- & 10.9 & 7.5 & 703 & 8.2 \\
\hline 04160900 & 06-04-2002 & 815 & 41 & -- & 6.7 & 7.7 & 624 & 15.1 \\
\hline 04160900 & $07-09-2002$ & 1235 & 12 & -- & -- & 7.9 & 773 & -- \\
\hline 04160900 & 08-20-2002 & 1130 & -- & -- & -- & 7.9 & 870 & -- \\
\hline 04160900 & $12-12-2002$ & 1115 & 24 & -- & 12 & 7.8 & 721 & 1.4 \\
\hline 04160900 & 02-03-2003 & 1000 & 6.4 & -- & 12.1 & 7.6 & 924 & 1.4 \\
\hline 04160900 & 05-01-2003 & 1030 & 73 & -- & 8.9 & 7.7 & 688 & 13.6 \\
\hline 04160900 & $06-11-2003$ & 1045 & 68 & -- & 8.8 & 8.0 & 735 & 18.3 \\
\hline 04160900 & $07-22-2003$ & 1430 & -- & -- & -- & 7.9 & 815 & -- \\
\hline 04160900 & 08-05-2003 & 2000 & 3 & 549 & 6.7 & 7.6 & 844 & 22.5 \\
\hline 04161000 & $01-22-2002$ & 1355 & 103 & -- & -- & 7.8 & 922 & 3.7 \\
\hline 04161000 & $03-11-2002$ & 1345 & 159 & -- & 15.3 & 7.3 & 1070 & 3.9 \\
\hline 04161000 & 04-29-2002 & 1145 & 140 & -- & -- & 8.2 & 850 & -- \\
\hline 04161000 & 06-04-2002 & 1200 & 116 & -- & 7.1 & 7.7 & 626 & 15.5 \\
\hline 04161000 & 07-09-2002 & 1332 & 23 & -- & 7.5 & 7.2 & 1140 & 21.2 \\
\hline 04161000 & $08-28-2002$ & 1345 & 22 & 193 & 8.5 & 7.4 & 1010 & 22.0 \\
\hline 04161000 & $12-12-2002$ & 1300 & 90 & -- & 13.4 & 8.3 & 785 & 2.7 \\
\hline 04161000 & 02-03-2003 & 1400 & 35 & -- & 13.5 & 7.5 & 1420 & 4.9 \\
\hline 04161000 & 04-30-2003 & 1730 & 73 & -- & 11.2 & 7.8 & 1040 & 14.5 \\
\hline 04161000 & $06-11-2003$ & 1515 & 115 & -- & 9.3 & 7.9 & 834 & 19.5 \\
\hline 04161000 & $07-23-2003$ & 1600 & 21 & 360 & 9.8 & 7.8 & 1060 & 21.4 \\
\hline 04161000 & $08-05-2003$ & 1415 & 21 & 523 & 9 & 7.7 & 1090 & 22.3 \\
\hline 04161000 & 09-08-2003 & 1500 & 23 & 338 & 9.7 & 7.9 & 952 & 21.9 \\
\hline 04161540 & 09-19-2001 & 1130 & 137 & -- & 8.7 & 7.8 & 559 & 17.1 \\
\hline 04161540 & $11-15-2001$ & 1130 & 93 & -- & 11.5 & 7.8 & 670 & 9.9 \\
\hline 04161540 & $01-24-2002$ & 1100 & 66 & -- & 12.1 & 7.6 & 907 & 3.2 \\
\hline 04161540 & $03-12-2002$ & 1110 & 114 & -- & 14.4 & 8.2 & 738 & 2.7 \\
\hline 04161540 & 05-01-2002 & 940 & 49 & -- & 11.3 & 8.3 & 747 & 10.0 \\
\hline 04161540 & 06-06-2002 & 1230 & 98 & -- & 9 & 8.1 & 624 & 15.5 \\
\hline 04161540 & 07-09-2002 & 1150 & 15 & -- & 8.4 & 7.9 & 778 & 19.5 \\
\hline 04161540 & 08-28-2002 & 1230 & 13 & 169 & 9.3 & 7.8 & 749 & 17.7 \\
\hline 04161540 & $11-11-2002$ & 1215 & 30 & 166 & 9.8 & 7.8 & 733 & 10.5 \\
\hline 04161540 & $12-11-2002$ & 1115 & 9 & -- & -- & 8.0 & 877 & 0.3 \\
\hline 04161540 & $01-28-2003$ & 1635 & 99 & -- & 12.9 & 7.8 & 846 & -0.2 \\
\hline 04161540 & 02-02-2003 & 1545 & 53 & -- & 15.1 & 7.9 & 893 & -0.2 \\
\hline 04161540 & 04-22-2003 & 1230 & 47 & 324 & 10.5 & 8.0 & 748 & 8.8 \\
\hline
\end{tabular}


Table 9.Results of synoptic stream-water-quality sample analysis in and around Oakland County, Michigan between September 2001 and September 2003- continued

$[\mathrm{mg} / \mathrm{L}$, milligrams per liter; $\mathrm{mg} / \mathrm{L}$, micrograms per liter; $\mathrm{mS} / \mathrm{cm}$, microsiemens per centimeter; wf, filtered water; $\mathrm{mL}$, milliliter]

\begin{tabular}{|c|c|c|c|c|c|c|c|c|}
\hline 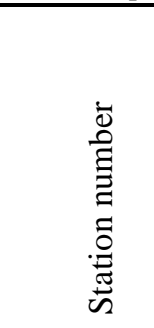 & 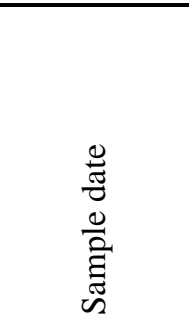 & 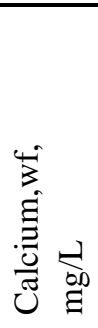 & 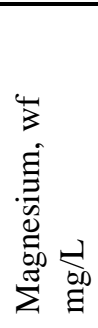 & 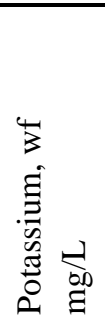 & 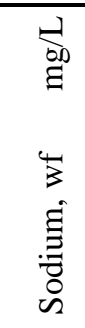 & 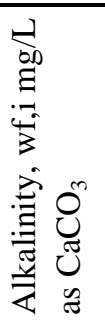 & 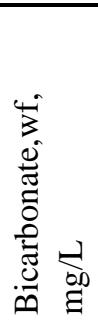 & 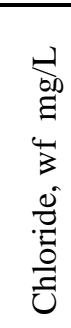 \\
\hline 04160900 & 09-17-2001 & 81.9 & 22.4 & 2.2 & 63.2 & 134 & 163 & 123 \\
\hline 04160900 & $11-14-2001$ & 58.1 & 21.1 & 2.3 & 53.7 & 229 & 279 & 113 \\
\hline 04160900 & $01-22-2002$ & 60.0 & 20.6 & 2.6 & 51.2 & 282 & 344 & 107 \\
\hline 04160900 & 03-11-2002 & 64.2 & 20.2 & 2.0 & 49.8 & 212 & 259 & 100 \\
\hline 04160900 & 04-29-2002 & 66.3 & 20.4 & 2.3 & 51.8 & 184 & 224 & 100 \\
\hline 04160900 & 06-04-2002 & 57.7 & 17.2 & 1.9 & 44.5 & 154 & 188 & 87 \\
\hline 04160900 & 07-09-2002 & 63.3 & 22.3 & 1.7 & 61.3 & 186 & 227 & 117 \\
\hline 04160900 & $08-20-2002$ & 81.4 & 23.6 & 2.2 & 66.9 & 271 & -- & 133 \\
\hline 04160900 & $12-12-2002$ & 57.4 & 21.9 & 2.1 & 54.5 & 176 & 215 & 113 \\
\hline 04160900 & 02-03-2003 & 67.7 & 23.5 & 2.3 & 83 & 230 & -- & 159 \\
\hline 04160900 & 05-01-2003 & 65.5 & 22.5 & 2.5 & 57.9 & 170 & -- & 108 \\
\hline 04160900 & 06-11-2003 & 61.5 & 21.3 & 2.2 & 56.8 & 174 & 212 & 110 \\
\hline 04160900 & $07-22-2003$ & 70.8 & 22.6 & 2.2 & 66.7 & -- & -- & 132 \\
\hline 04160900 & 08-05-2003 & 78.8 & 24.2 & 2.3 & 73.6 & 208 & 254 & 134 \\
\hline 04161000 & $01-22-2002$ & 61.6 & 19.8 & 3.7 & 92 & 168 & 205 & 166 \\
\hline 04161000 & 03-11-2002 & 65.1 & 19.1 & 3.1 & 111 & -- & -- & 197 \\
\hline 04161000 & 04-29-2002 & 65.4 & 18.9 & 3.5 & 82.1 & 180 & 220 & 147 \\
\hline 04161000 & 06-04-2002 & 44.2 & 10.7 & 3.9 & 61.1 & 134 & 163 & 108 \\
\hline 04161000 & 07-09-2002 & 75.9 & 19.8 & 7.9 & 127 & 150 & 183 & 213 \\
\hline 04161000 & 08-28-2002 & 72.4 & 17.5 & 9.4 & 104 & 141 & 172 & 175 \\
\hline 04161000 & $12-12-2002$ & 55.0 & 20.6 & 3.6 & 70.7 & 152 & 185 & 135 \\
\hline 04161000 & 02-03-2003 & 66.6 & 18.0 & 6.9 & 181 & 170 & 207 & 301 \\
\hline 04161000 & 04-30-2003 & 65.8 & 19.9 & 5.0 & 127 & 143 & 174 & 222 \\
\hline 04161000 & 06-11-2003 & 58.0 & 18.6 & 3.8 & 81.4 & 158 & 193 & 148 \\
\hline 04161000 & $07-23-2003$ & 69.9 & 16.6 & 9.5 & 120 & 134 & 163 & 194 \\
\hline 04161000 & 08-05-2003 & 73.8 & 17.6 & 9.1 & 119 & 144 & 176 & 194 \\
\hline 04161000 & 09-08-2003 & 65.0 & 13.9 & 11.2 & 103 & -- & -- & 176 \\
\hline 04161540 & 09-19-2001 & 52.4 & 14.5 & 3.7 & 35 & 170 & 207 & 71 \\
\hline 04161540 & $11-15-2001$ & 70.9 & 22.0 & 2.3 & 35.1 & 194 & 237 & 75 \\
\hline 04161540 & 01-24-2002 & 81.2 & 23.6 & 2.3 & 68.9 & 216 & 264 & 129 \\
\hline 04161540 & 03-12-2002 & 73.2 & 21.2 & 2.2 & 43.6 & -- & -- & 84 \\
\hline 04161540 & 05-01-2002 & 79.1 & 23.4 & 1.9 & 41.2 & 230 & 281 & 83 \\
\hline 04161540 & 06-06-2002 & 67.3 & 20.6 & 2.0 & 33.6 & 190 & 232 & 67 \\
\hline 04161540 & 07-09-2002 & 83.2 & 25.9 & 2.0 & 42.9 & 244 & 297 & 86 \\
\hline 04161540 & 08-28-2002 & 82.8 & 25.7 & 2.0 & 43.5 & 141 & 172 & 91 \\
\hline 04161540 & $11-11-2002$ & 77.7 & 22.9 & 3.6 & 41.1 & 272 & -- & 93 \\
\hline 04161540 & $12-11-2002$ & 94.5 & 27.8 & 2.0 & 51 & 250 & 305 & 106 \\
\hline 04161540 & $01-28-2003$ & 84.3 & 28.0 & 2.1 & 60.2 & 254 & 305 & 112 \\
\hline 04161540 & 02-02-2003 & 78.6 & 24.7 & 2.1 & 72 & 252 & 302 & 142 \\
\hline 04161540 & 04-22-2003 & 76.6 & 23.3 & 2.5 & 47.7 & 206 & 251 & 97 \\
\hline
\end{tabular}


Table 9.Results of synoptic stream-water-quality sample analysis in and around Oakland County, Michigan between September 2001 and September 2003- continued

[mg/L, milligrams per liter; $\mathrm{mg} / \mathrm{L}$, micrograms per liter; $\mathrm{mS} / \mathrm{cm}$, microsiemens per centimeter; wf, filtered water; $\mathrm{mL}$, milliliter]

\begin{tabular}{|c|c|c|c|c|c|c|c|c|c|}
\hline 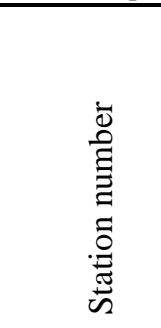 & 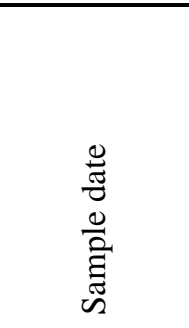 & 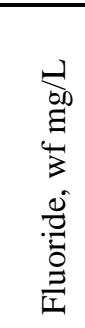 & 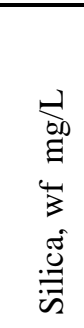 & 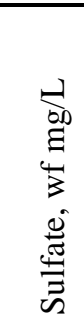 & 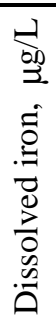 & 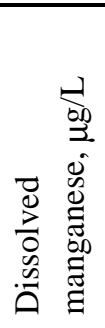 & 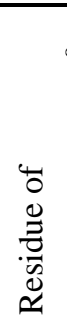 & 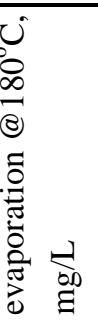 & 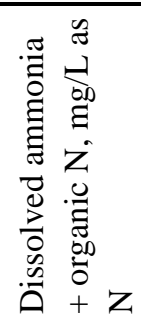 \\
\hline$\overline{04160900}$ & 09-17-2001 & E.1 & 8.3 & 24.0 & 17 & 79.1 & & 488 & 0.41 \\
\hline 04160900 & $11-14-2001$ & 0.1 & 6.5 & 27.3 & 35 & 16.5 & & 404 & 0.38 \\
\hline 04160900 & $01-22-2002$ & 0.1 & 5.3 & 26.4 & 41 & 12.4 & & 434 & 0.4 \\
\hline 04160900 & 03-11-2002 & 0.2 & 4.9 & 25.5 & 34 & 14 & & 402 & 0.37 \\
\hline 04160900 & 04-29-2002 & 0.1 & 3.4 & 24.5 & 63 & 18.4 & & 411 & 0.39 \\
\hline 04160900 & 06-04-2002 & 0.1 & 3.0 & 23.0 & 76 & 28.3 & & 396 & 0.54 \\
\hline 04160900 & 07-09-2002 & $<.10$ & 5.9 & 23.6 & 70 & 49.3 & & 454 & 0.49 \\
\hline 04160900 & 08-20-2002 & 0.2 & 8.8 & 21.7 & 46 & 60.3 & & 535 & 0.51 \\
\hline 04160900 & $12-12-2002$ & $<.17$ & 5.6 & 24.6 & 31 & 17.3 & & 413 & 0.39 \\
\hline 04160900 & 02-03-2003 & 0.2 & 6.3 & 27.4 & 60 & 46.4 & & 523 & -- \\
\hline 04160900 & $05-01-2003$ & $<.17$ & 3.5 & 29.3 & 51 & 21.1 & & 409 & 0.45 \\
\hline 04160900 & 06-11-2003 & $<.2$ & 1.0 & 28.0 & 32 & 9.6 & & 430 & 0.45 \\
\hline 04160900 & $07-22-2003$ & $<.2$ & 7.2 & 25.2 & 28 & 82.9 & & 501 & 0.49 \\
\hline 04160900 & $08-05-2003$ & $<.2$ & 8.0 & 23.0 & 20 & 61 & & 530 & 0.46 \\
\hline 04161000 & $01-22-2002$ & 0.2 & 4.5 & 33.1 & 44 & 21 & & 524 & 0.52 \\
\hline 04161000 & 03-11-2002 & 0.3 & 4.6 & 34.0 & 31 & 23.4 & & 580 & 0.45 \\
\hline 04161000 & 04-29-2002 & 0.3 & 3.1 & 32.6 & 41 & 19 & & 506 & 0.49 \\
\hline 04161000 & 06-04-2002 & 0.2 & 2.9 & 27.0 & 35 & 34 & & 381 & 0.85 \\
\hline 04161000 & 07-09-2002 & 0.8 & 5.5 & 55.1 & 57 & 30.9 & & 675 & 0.71 \\
\hline 04161000 & 08-28-2002 & 0.9 & 6.8 & 54.0 & 44 & 23.1 & & 609 & 0.73 \\
\hline 04161000 & $12-12-2002$ & 0.3 & 2.8 & 31.0 & 36 & 13.5 & & 433 & 0.59 \\
\hline 04161000 & 02-03-2003 & 0.7 & 5.1 & 46.6 & 61 & 64.7 & & 784 & 2.4 \\
\hline 04161000 & 04-30-2003 & 0.3 & 2.1 & 40.6 & 58 & 35.5 & & 610 & 1 \\
\hline 04161000 & 06-11-2003 & 0.3 & 2.2 & 33.3 & 42 & 15.5 & & 475 & 0.58 \\
\hline 04161000 & 07-23-2003 & 1.1 & 6.4 & 53.9 & 43 & 25.4 & & 611 & 0.86 \\
\hline 04161000 & $08-05-2003$ & 0.8 & 6.9 & 52.4 & 54 & 29.1 & & 675 & 0.86 \\
\hline 04161000 & 09-08-2003 & 1.0 & 6.4 & 48.1 & 57 & 17.3 & & 578 & 0.87 \\
\hline 04161540 & 09-19-2001 & 0.2 & 5.1 & 27.1 & 22 & 21.7 & & 314 & 0.64 \\
\hline 04161540 & $11-15-2001$ & 0.2 & 7.1 & 35.2 & 43 & 25.1 & & 388 & 0.32 \\
\hline 04161540 & 01-24-2002 & 0.2 & 7.0 & 36.1 & 44 & 39.1 & & 526 & 0.33 \\
\hline 04161540 & 03-12-2002 & 0.2 & 5.6 & 33.8 & 41 & 24.8 & & 416 & 0.35 \\
\hline 04161540 & 05-01-2002 & 0.2 & 3.3 & 32.1 & 90 & 28.1 & & 433 & 0.33 \\
\hline 04161540 & 06-06-2002 & 0.2 & 4.6 & 26.5 & 48 & 20.2 & & 412 & 0.52 \\
\hline 04161540 & 07-09-2002 & 0.2 & 8.4 & 31.9 & 46 & 21.2 & & 476 & 0.23 \\
\hline 04161540 & 08-28-2002 & 0.3 & 9.4 & 29.3 & 16 & 17.8 & & 486 & 0.27 \\
\hline 04161540 & $11-11-2002$ & 0.2 & 9.1 & 34.5 & 98 & 27.9 & & 434 & 0.3 \\
\hline 04161540 & $12-11-2002$ & 0.3 & 10.7 & 37.2 & 52 & 28.5 & & 532 & 0.2 \\
\hline 04161540 & $01-28-2003$ & 0.2 & 9.2 & 35.6 & 63 & 26.9 & & 498 & 0.3 \\
\hline 04161540 & 02-02-2003 & 0.2 & 8.2 & 34.8 & 61 & 31.5 & & 529 & 0.28 \\
\hline 04161540 & 04-22-2003 & 0.2 & 6.0 & 42.2 & 75 & 35.9 & & 462 & 0.4 \\
\hline
\end{tabular}


Table 9.Results of synoptic stream-water-quality sample analysis in and around Oakland County, Michigan between September 2001 and September 2003- continued

$[\mathrm{mg} / \mathrm{L}$, milligrams per liter; $\mathrm{mg} / \mathrm{L}$, micrograms per liter; $\mathrm{mS} / \mathrm{cm}$, microsiemens per centimeter; wf, filtered water; $\mathrm{mL}$, milliliter]

\begin{tabular}{|c|c|c|c|c|c|c|c|c|}
\hline 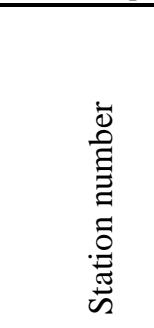 & 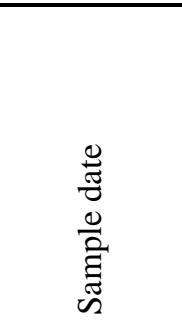 & 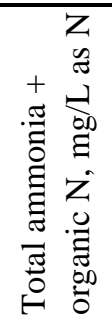 & 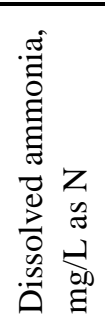 & 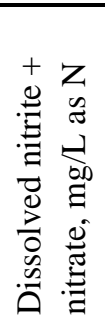 & 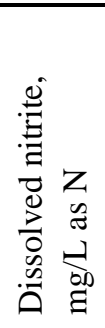 & 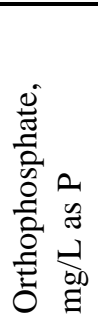 & 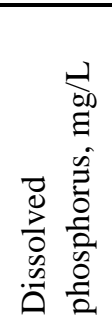 & 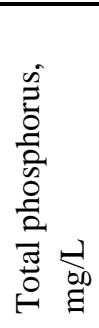 \\
\hline 04160900 & 09-17-2001 & 0.42 & 0.15 & 0.13 & E.005 & E.01 & 0.021 & 0.019 \\
\hline 04160900 & $11-14-2001$ & 0.47 & E. 03 & 0.06 & E.004 & $<.02$ & E.004 & 0.004 \\
\hline 04160900 & $01-22-2002$ & 0.43 & 0.05 & 0.14 & E.006 & $<.02$ & E.004 & E.003 \\
\hline 04160900 & 03-11-2002 & 0.54 & 0.04 & 0.15 & E.004 & $<.02$ & E.003 & $<.004$ \\
\hline 04160900 & 04-29-2002 & 1 & E.04 & 0.14 & E.007 & $<.02$ & 0.005 & E.05 \\
\hline 04160900 & 06-04-2002 & 0.66 & 0.11 & 0.23 & E.007 & $<.02$ & 0.008 & $<.004$ \\
\hline 04160900 & 07-09-2002 & 0.45 & 0.08 & 0.07 & E.007 & $<.02$ & 0.009 & $<.004$ \\
\hline 04160900 & $08-20-2002$ & 0.43 & 0.18 & 0.13 & E.007 & $<.02$ & E.004 & 0.013 \\
\hline 04160900 & $12-12-2002$ & 0.48 & 0.08 & 0.07 & E.005 & $<.02$ & 0.005 & 0.011 \\
\hline 04160900 & 02-03-2003 & -- & -- & -- & -- & -- & E.003 & 0.008 \\
\hline 04160900 & 05-01-2003 & 0.51 & 0.07 & 0.12 & E.005 & $<.02$ & 0.005 & 0.018 \\
\hline 04160900 & 06-11-2003 & 0.56 & E.03 & 0.08 & $<.008$ & $<.02$ & E.004 & 0.017 \\
\hline 04160900 & $07-22-2003$ & 0.57 & 0.14 & 0.06 & E.004 & $<.02$ & 0.005 & 0.017 \\
\hline 04160900 & $08-05-2003$ & 0.58 & 0.13 & 0.09 & E.006 & $<.02$ & E.004 & 0.023 \\
\hline 04161000 & $01-22-2002$ & 0.52 & 0.05 & 1.9 & 0.008 & 0.03 & 0.035 & 0.007 \\
\hline 04161000 & 03-11-2002 & 0.39 & 0.04 & 2.2 & E.007 & 0.02 & 0.027 & $<.004$ \\
\hline 04161000 & 04-29-2002 & 0.58 & E.02 & 1.9 & 0.016 & 0.02 & 0.04 & 0.06 \\
\hline 04161000 & 06-04-2002 & 1.1 & 0.28 & 3.05 & 0.036 & 0.06 & 0.082 & 0.02 \\
\hline 04161000 & 07-09-2002 & 0.75 & 0.07 & 10.3 & 0.052 & 0.16 & 0.2 & 0.21 \\
\hline 04161000 & $08-28-2002$ & 0.68 & 0.07 & 11.1 & 0.05 & 0.38 & 0.43 & 0.46 \\
\hline 04161000 & $12-12-2002$ & 0.71 & 0.12 & 1.52 & E.005 & 0.07 & 0.091 & 0.111 \\
\hline 04161000 & 02-03-2003 & 2.6 & 1.82 & 6.43 & 0.043 & 0.22 & 0.25 & 0.26 \\
\hline 04161000 & 04-30-2003 & 1.5 & 0.29 & 3.98 & 0.055 & 0.02 & 0.044 & 0.107 \\
\hline 04161000 & $06-11-2003$ & 0.67 & $<.04$ & 2.25 & 0.024 & 0.04 & 0.062 & 0.095 \\
\hline 04161000 & $07-23-2003$ & 0.94 & 0.06 & 11.7 & 0.03 & 0.43 & 0.46 & 0.49 \\
\hline 04161000 & 08-05-2003 & 0.88 & 0.06 & 10.8 & 0.035 & 0.55 & 0.57 & 0.6 \\
\hline 04161000 & 09-08-2003 & 0.85 & $<.41$ & 11.4 & 0.029 & 0.44 & 0.53 & 0.54 \\
\hline 04161540 & 09-19-2001 & 1.1 & 0.09 & 0.45 & 0.015 & $<.02$ & 0.023 & 0.064 \\
\hline 04161540 & $11-15-2001$ & 0.4 & $<.04$ & 0.24 & E.004 & $<.02$ & 0.004 & 0.004 \\
\hline 04161540 & 01-24-2002 & 0.38 & $<.04$ & 0.43 & E.004 & $<.02$ & E.004 & E.003 \\
\hline 04161540 & $03-12-2002$ & 0.39 & $<.04$ & 0.32 & E.005 & $<.02$ & E.004 & $<.004$ \\
\hline 04161540 & 05-01-2002 & 0.36 & $<.04$ & 0.15 & E.004 & $<.02$ & 0.004 & $<.004$ \\
\hline 04161540 & 06-06-2002 & 0.69 & 0.05 & 0.21 & 0.009 & $<.02$ & 0.011 & 0.004 \\
\hline 04161540 & 07-09-2002 & 0.27 & $<.04$ & 0.32 & E.006 & $<.02$ & 0.008 & $<.06$ \\
\hline 04161540 & $08-28-2002$ & 0.27 & $<.04$ & 0.28 & $<.008$ & $<.02$ & 0.007 & 0.018 \\
\hline 04161540 & $11-11-2002$ & 0.42 & $<.04$ & 0.11 & E.005 & $<.02$ & 0.006 & 0.026 \\
\hline 04161540 & $12-11-2002$ & 0.24 & E.04 & 0.31 & $<.008$ & $<.02$ & E.003 & 0.01 \\
\hline 04161540 & $01-28-2003$ & 0.32 & 0.05 & 0.23 & E.006 & $<.02$ & 0.006 & 0.01 \\
\hline 04161540 & 02-02-2003 & 0.29 & 0.06 & 0.21 & E.006 & $<.02$ & 0.005 & 0.01 \\
\hline 04161540 & 04-22-2003 & 0.44 & E.03 & 0.25 & E.005 & $<.02$ & 0.008 & 0.014 \\
\hline
\end{tabular}


Table 9.Results of synoptic stream-water-quality sample analysis in and around Oakland County, Michigan between September 2001 and September 2003- continued

$[\mathrm{mg} / \mathrm{L}$, milligrams per liter; $\mathrm{mg} / \mathrm{L}$, micrograms per liter; $\mathrm{mS} / \mathrm{cm}$, microsiemens per centimeter; wf, filtered water; $\mathrm{mL}$, milliliter]

\begin{tabular}{|c|c|c|c|c|c|}
\hline 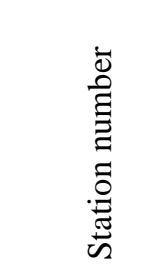 & 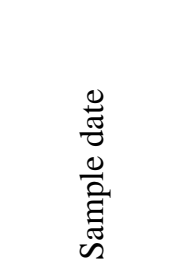 & 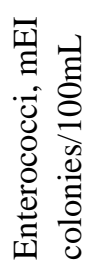 & 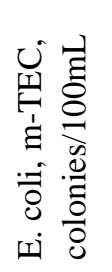 & 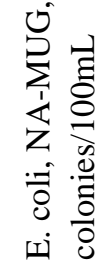 & 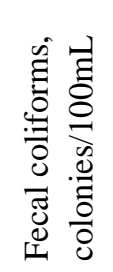 \\
\hline 04160900 & $09-17-2001$ & -- & -- & -- & -- \\
\hline 04160900 & 11-14-2001 & -- & -- & -- & -- \\
\hline 04160900 & $01-22-2002$ & -- & -- & -- & -- \\
\hline 04160900 & $03-11-2002$ & -- & 13 & -- & -- \\
\hline 04160900 & 04-29-2002 & -- & 120 & -- & -- \\
\hline 04160900 & 06-04-2002 & -- & 9700 & -- & -- \\
\hline 04160900 & 07-09-2002 & -- & 150 & -- & -- \\
\hline 04160900 & $08-20-2002$ & -- & -- & -- & -- \\
\hline 04160900 & $12-12-2002$ & -- & -- & -- & -- \\
\hline 04160900 & 02-03-2003 & -- & -- & -- & -- \\
\hline 04160900 & 05-01-2003 & -- & -- & -- & -- \\
\hline 04160900 & $06-11-2003$ & -- & 190 & -- & -- \\
\hline 04160900 & $07-22-2003$ & -- & -- & -- & -- \\
\hline 04160900 & 08-05-2003 & E906 & -- & E1700 & E3700 \\
\hline 04161000 & $01-22-2002$ & -- & -- & -- & -- \\
\hline 04161000 & $03-11-2002$ & -- & 43 & -- & -- \\
\hline 04161000 & 04-29-2002 & -- & 97 & -- & -- \\
\hline 04161000 & 06-04-2002 & -- & 3100 & -- & -- \\
\hline 04161000 & 07-09-2002 & -- & 150 & -- & -- \\
\hline 04161000 & $08-28-2002$ & -- & 800 & -- & -- \\
\hline 04161000 & $12-12-2002$ & -- & -- & -- & -- \\
\hline 04161000 & 02-03-2003 & -- & 4200 & -- & -- \\
\hline 04161000 & 04-30-2003 & -- & 140 & -- & -- \\
\hline 04161000 & $06-11-2003$ & -- & 200 & -- & -- \\
\hline 04161000 & $07-23-2003$ & -- & -- & -- & -- \\
\hline 04161000 & 08-05-2003 & E100 & -- & E110 & E1620 \\
\hline 04161000 & 09-08-2003 & $\mathrm{E} 400$ & -- & E320 & E1030 \\
\hline 04161540 & 09-19-2001 & -- & -- & -- & -- \\
\hline 04161540 & $11-15-2001$ & -- & -- & -- & -- \\
\hline 04161540 & 01-24-2002 & -- & -- & -- & -- \\
\hline 04161540 & $03-12-2002$ & -- & 8 & -- & -- \\
\hline 04161540 & 05-01-2002 & -- & 57 & -- & -- \\
\hline 04161540 & 06-06-2002 & -- & 590 & -- & -- \\
\hline 04161540 & 07-09-2002 & -- & 2100 & -- & -- \\
\hline 04161540 & 08-28-2002 & -- & 580 & -- & -- \\
\hline 04161540 & $11-11-2002$ & -- & 800 & -- & -- \\
\hline 04161540 & $12-11-2002$ & -- & -- & -- & -- \\
\hline 04161540 & $01-28-2003$ & -- & -- & -- & -- \\
\hline 04161540 & 02-02-2003 & -- & 35 & -- & -- \\
\hline 04161540 & $04-22-2003$ & -- & 20 & -- & -- \\
\hline
\end{tabular}


Table 9.Results of synoptic stream-water-quality sample analysis in and around Oakland County, Michigan between September 2001 and September 2003- continued

$[\mathrm{mg} / \mathrm{L}$, milligrams per liter; $\mathrm{mg} / \mathrm{L}$, micrograms per liter; $\mathrm{mS} / \mathrm{cm}$, microsiemens per centimeter; wf, filtered water; $\mathrm{mL}$, milliliter]

\begin{tabular}{|c|c|c|c|c|c|c|c|c|}
\hline 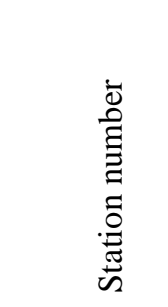 & 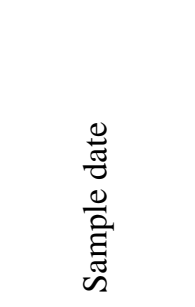 & 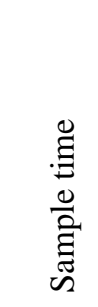 & 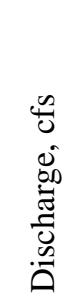 & 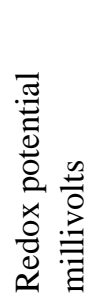 & 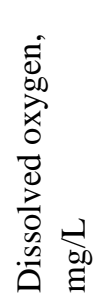 & 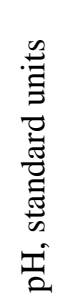 & 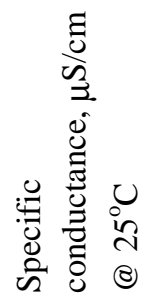 & 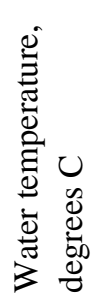 \\
\hline 04161540 & $07-23-2003$ & 1145 & 14 & 353 & 8.8 & 8.0 & 763 & 17.3 \\
\hline 04161540 & $08-05-2003$ & 1545 & 24 & 537 & 8.2 & 8.0 & 678 & 20.0 \\
\hline 04161540 & 09-09-2003 & 1245 & 8 & 338 & 10.4 & 8.2 & 801 & 16.4 \\
\hline 04161580 & $12-11-2002$ & 1300 & 8.1 & -- & -- & 7.4 & 637 & 1.0 \\
\hline 04161580 & $06-11-2003$ & 1415 & 15 & -- & 9.6 & 8.1 & 575 & 18.7 \\
\hline 04161580 & 08-05-2003 & 1630 & 3.2 & 415 & 8.5 & 8.3 & 634 & 23.5 \\
\hline 04161580 & 09-09-2003 & 1545 & 1.7 & 180 & 9.6 & 8.3 & 611 & 19.8 \\
\hline 04161810 & $09-19-2001$ & 930 & 228 & -- & 8.8 & 7.7 & 801 & 17.2 \\
\hline 04161810 & $11-15-2001$ & 945 & 144 & -- & 10.7 & 7.5 & 723 & 10.7 \\
\hline 04161810 & $01-24-2002$ & 1250 & -- & -- & 13.7 & 7.9 & 1190 & 3.2 \\
\hline 04161810 & 03-12-2002 & 1245 & 292 & -- & 13.8 & 7.8 & 1070 & 4.4 \\
\hline 04161810 & 05-01-2002 & 1100 & 235 & -- & 11.4 & 8.4 & 864 & 11.0 \\
\hline 04161810 & 06-06-2002 & 1030 & 346 & -- & 9.7 & 8.1 & 734 & 16.2 \\
\hline 04161810 & 07-09-2002 & 1033 & 52 & -- & 7.9 & 8.0 & 957 & 22.2 \\
\hline 04161810 & 08-21-2002 & 1100 & 50 & 165 & 8.5 & 8.1 & 848 & 19.4 \\
\hline 04161810 & $11-11-2002$ & 1100 & 139 & 169 & 10.2 & 7.8 & 635 & 10.8 \\
\hline 04161810 & $12-11-2002$ & 1000 & 125 & 166 & 14.2 & 7.0 & 871 & 0.5 \\
\hline 04161810 & $01-28-2003$ & 1600 & 49 & -- & 13.7 & 8.0 & 1100 & -0.2 \\
\hline 04161810 & $02-02-2003$ & 1500 & -- & -- & 16.2 & 8.5 & 2580 & -0.3 \\
\hline 04161810 & $05-01-2003$ & 1200 & 456 & -- & 9.4 & 7.6 & 835 & 13.5 \\
\hline 04161810 & $06-11-2003$ & 1215 & 235 & -- & 9.5 & 7.9 & 871 & 18.2 \\
\hline 04161810 & $07-23-2003$ & 1300 & 59 & -- & 9.9 & 8.0 & 944 & 20.1 \\
\hline 04161810 & $08-05-2003$ & 1500 & 63 & 467 & 9.1 & 8.2 & 853 & 22.0 \\
\hline 04161810 & 09-09-2003 & 1415 & 34 & 331 & 10.3 & 8.5 & 937 & 19.3 \\
\hline 04166000 & 09-18-2001 & 1345 & 8 & -- & 8.3 & 7.9 & 952 & 18.9 \\
\hline 04166000 & $11-15-2001$ & 1400 & 55 & -- & 11 & 7.7 & 1120 & 9.7 \\
\hline 04166000 & 01-24-2002 & 910 & 28 & -- & 12.8 & 7.8 & 1550 & 2.5 \\
\hline 04166000 & 03-12-2002 & 915 & 43 & -- & 13.8 & 8.6 & 1680 & 2.0 \\
\hline 04166000 & 05-01-2002 & 745 & 23 & -- & 10.3 & 8.3 & 1210 & 10.3 \\
\hline 04166000 & 06-06-2002 & 815 & 20 & -- & 8.1 & 7.8 & 1040 & 16.4 \\
\hline 04166000 & 07-09-2002 & 843 & 4.8 & -- & 5.8 & 7.7 & 1150 & 25.0 \\
\hline 04166000 & 08-28-2002 & 1000 & 5.4 & 178 & 6.2 & 7.6 & 1080 & 21.8 \\
\hline 04166000 & 09-03-2002 & 1030 & 4.2 & 176 & 5 & 7.5 & 1090 & 23.3 \\
\hline 04166000 & $12-12-2002$ & 1400 & 7.2 & -- & 13.1 & 8.2 & 1540 & 0.8 \\
\hline 04166000 & 02-02-2003 & 1330 & 8 & -- & 14.8 & 7.8 & 2120 & 0.3 \\
\hline 04166000 & 04-30-2003 & 1630 & 14 & -- & 10.4 & 8.0 & 1540 & 14.7 \\
\hline 04166000 & $05-20-2003$ & 1230 & 22 & -- & 8.1 & 8.0 & 1320 & 17.8 \\
\hline 04166000 & $07-23-2003$ & 1500 & 6.1 & 375 & 7.6 & 8.1 & 1160 & 21.9 \\
\hline 04166000 & 08-05-2003 & 1315 & 8.9 & 516 & 7.3 & 8.1 & 1160 & 22.5 \\
\hline 04166000 & 09-08-2003 & 1400 & 3.6 & 320 & 8.5 & 8.3 & 1110 & 20.9 \\
\hline
\end{tabular}


Table 9.Results of synoptic stream-water-quality sample analysis in and around Oakland County, Michigan between September 2001 and September 2003- continued

$[\mathrm{mg} / \mathrm{L}$, milligrams per liter; $\mathrm{mg} / \mathrm{L}$, micrograms per liter; $\mathrm{mS} / \mathrm{cm}$, microsiemens per centimeter; wf, filtered water; $\mathrm{mL}$, milliliter]

\begin{tabular}{|c|c|c|c|c|c|c|c|c|}
\hline 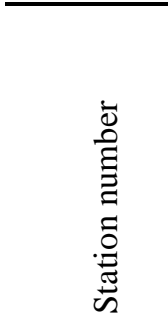 & 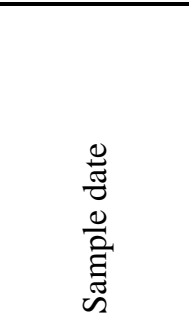 & 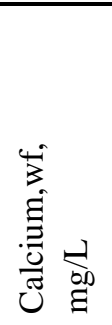 & 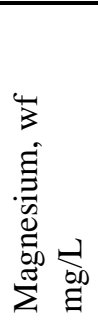 & 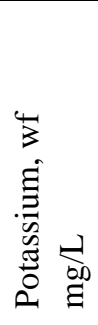 & 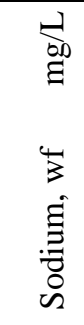 & 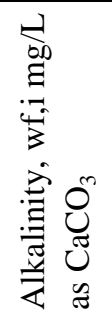 & 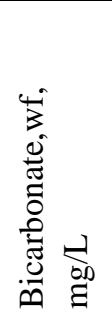 & 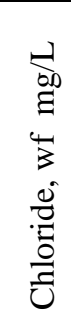 \\
\hline 04161540 & $07-23-2003$ & 73.7 & 20.4 & 2.9 & 55.7 & 174 & 212 & 107 \\
\hline 04161540 & $08-05-2003$ & 65.6 & 18.8 & 3.6 & 48.5 & 190 & 232 & 93 \\
\hline 04161540 & 09-09-2003 & 80.7 & 26.0 & 2.3 & 46.2 & 252 & 307 & 103 \\
\hline 04161580 & $12-11-2002$ & 67.5 & 27.7 & 1.6 & 23.4 & 212 & -- & 53 \\
\hline 04161580 & 06-11-2003 & 64.0 & 23.4 & 1.5 & 23.4 & 204 & -- & 52 \\
\hline 04161580 & 08-05-2003 & 69.3 & 27.1 & 1.8 & 29.7 & 232 & -- & 59 \\
\hline 04161580 & 09-09-2003 & 62.3 & 27.7 & 1.9 & 25.9 & 216 & -- & 58 \\
\hline 04161810 & 09-19-2001 & 63.3 & 18.6 & 4.6 & 64.2 & 152 & 185 & 119 \\
\hline 04161810 & $11-15-2001$ & 61.5 & 17.9 & 3.4 & 57.2 & 150 & 183 & 106 \\
\hline 04161810 & 01-24-2002 & 78.3 & 22.6 & 3.4 & 133 & -- & -- & 232 \\
\hline 04161810 & 03-12-2002 & 74.5 & 20.7 & 2.7 & 104 & -- & -- & 190 \\
\hline 04161810 & 05-01-2002 & 73.4 & 22.1 & 2.8 & 72.9 & 216 & 264 & 133 \\
\hline 04161810 & 06-06-2002 & 66.8 & 20.7 & 2.7 & 55.2 & 192 & 234 & 106 \\
\hline 04161810 & 07-09-2002 & 79.4 & 24.1 & 4.2 & 83.3 & 204 & 249 & 151 \\
\hline 04161810 & 08-21-2002 & 72.7 & 22.3 & 4.7 & 70.9 & 192 & 234 & 132 \\
\hline 04161810 & 11-11-2002 & 54.1 & 17.2 & 3.7 & 50.7 & 151 & 184 & 99 \\
\hline 04161810 & $12-11-2002$ & 63.0 & 22.6 & 3.4 & 76.8 & 166 & 202 & 145 \\
\hline 04161810 & 01-28-2003 & 79.9 & 25.0 & 4.4 & 117 & 214 & 261 & 198 \\
\hline 04161810 & $02-02-2003$ & 79.3 & 23.9 & 5.4 & 409 & 220 & 268 & 675 \\
\hline 04161810 & 05-01-2003 & 49.5 & 11.9 & 3.2 & 111 & 106 & 129 & 182 \\
\hline 04161810 & 06-11-2003 & 67.7 & 20.6 & 3.1 & 80.5 & 168 & 205 & 146 \\
\hline 04161810 & 07-23-2003 & 73.9 & 21.0 & 4.6 & 98.9 & 174 & 212 & 183 \\
\hline 04161810 & 08-05-2003 & 69.9 & 20.4 & 4.6 & 74.9 & 168 & 205 & 138 \\
\hline 04161810 & 09-09-2003 & 74.0 & 22.5 & 6.2 & 85.1 & 188 & 230 & 162 \\
\hline 04166000 & 09-18-2001 & 77.7 & 22.1 & 4.1 & 81.6 & 242 & 295 & 151 \\
\hline 04166000 & $11-15-2001$ & 103.0 & 26.0 & 3.8 & 91.9 & 232 & 283 & 179 \\
\hline 04166000 & 01-24-2002 & 111.0 & 28.2 & 3.4 & 157 & 256 & 312 & 291 \\
\hline 04166000 & 03-12-2002 & 96.2 & 22.2 & 3.2 & 202 & -- & -- & 354 \\
\hline 04166000 & 05-01-2002 & 93.5 & 25.2 & 3.2 & 119 & 274 & 334 & 219 \\
\hline 04166000 & 06-06-2002 & 86.5 & 24.1 & 3.5 & 95.6 & 208 & 254 & 182 \\
\hline 04166000 & 07-09-2002 & 81.0 & 27.5 & 4.4 & 116 & 205 & 250 & 217 \\
\hline 04166000 & 08-28-2002 & 84.3 & 25.3 & 3.9 & 94.2 & 210 & 256 & 189 \\
\hline 04166000 & 09-03-2002 & 90.2 & 28.0 & 3.9 & 97 & 216 & -- & 185 \\
\hline 04166000 & $12-12-2002$ & 124.0 & 33.4 & 4.0 & 137 & 289 & 353 & 280 \\
\hline 04166000 & $02-02-2003$ & 136.0 & 34.7 & 3.9 & 256 & 351 & 428 & 454 \\
\hline 04166000 & 04-30-2003 & 99.2 & 27.8 & 4.4 & 174 & 214 & 261 & 327 \\
\hline 04166000 & $05-20-2003$ & 101.0 & 26.2 & 4.3 & 126 & 222 & 271 & 253 \\
\hline 04166000 & 07-23-2003 & 92.1 & 26.2 & 4.2 & 120 & 224 & 273 & 225 \\
\hline 04166000 & 08-05-2003 & 99.0 & 31.1 & 4.5 & 106 & 232 & 283 & 202 \\
\hline 04166000 & 09-08-2003 & 88.6 & 28.5 & 4.6 & 102 & 198 & 242 & 202 \\
\hline
\end{tabular}


Table 9.Results of synoptic stream-water-quality sample analysis in and around Oakland County, Michigan between September 2001 and September 2003- continued

[mg/L, milligrams per liter; $\mathrm{mg} / \mathrm{L}$, micrograms per liter; $\mathrm{mS} / \mathrm{cm}$, microsiemens per centimeter; wf, filtered water; $\mathrm{mL}$, milliliter]

\begin{tabular}{|c|c|c|c|c|c|c|c|c|c|}
\hline 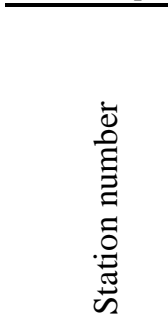 & 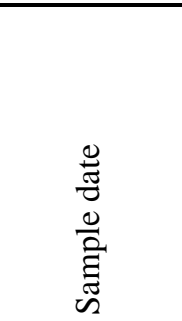 & 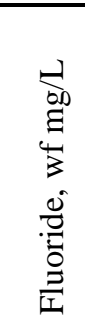 & 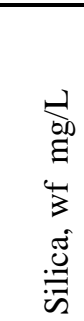 & 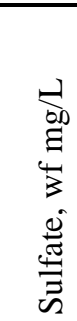 & 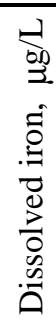 & 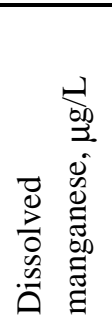 & 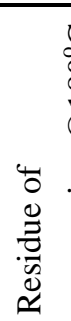 & 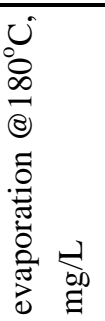 & 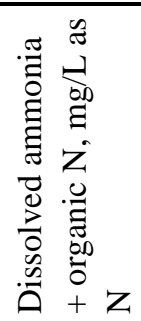 \\
\hline 04161540 & $07-23-2003$ & 0.3 & 9.9 & 32.2 & 31 & 28.8 & & 456 & 0.36 \\
\hline 04161540 & 08-05-2003 & 0.2 & 9.1 & 27.7 & 24 & 23.8 & & 443 & 0.38 \\
\hline 04161540 & 09-09-2003 & 0.3 & 10.5 & 33.0 & 42 & 25.4 & & 473 & 0.2 \\
\hline 04161580 & $12-11-2002$ & 0.2 & 9.5 & 34.1 & 28 & 15.1 & & 389 & 0.32 \\
\hline 04161580 & $06-11-2003$ & 0.2 & 5.4 & 26.3 & 97 & 21.1 & & 360 & 0.59 \\
\hline 04161580 & 08-05-2003 & 0.2 & 10.0 & 27.0 & 40 & 18.7 & & 420 & 0.31 \\
\hline 04161580 & 09-09-2003 & 0.2 & 11.2 & 29.8 & 64 & 26.8 & & 367 & 0.28 \\
\hline 04161810 & 09-19-2001 & 0.4 & 5.3 & 35.6 & 21 & 16.8 & & 449 & 0.56 \\
\hline 04161810 & $11-15-2001$ & 0.2 & 4.9 & 36.8 & 42 & 30.5 & & 418 & 0.51 \\
\hline 04161810 & $01-24-2002$ & 0.2 & 5.2 & 40.5 & 38 & 30.8 & & 680 & 0.39 \\
\hline 04161810 & $03-12-2002$ & 0.2 & 4.7 & 38.9 & 31 & 37.7 & & 572 & 0.39 \\
\hline 04161810 & 05-01-2002 & 0.2 & 1.3 & 35.8 & 58 & 21.3 & & 503 & 0.42 \\
\hline 04161810 & 06-06-2002 & 0.2 & 3.3 & 31.0 & 42 & 16.4 & & 488 & 0.52 \\
\hline 04161810 & 07-09-2002 & 0.4 & 5.1 & 45.1 & 42 & 22.7 & & 594 & 0.38 \\
\hline 04161810 & $08-21-2002$ & 0.5 & 7.2 & 40.2 & 32 & 12.3 & & 557 & 0.41 \\
\hline 04161810 & $11-11-2002$ & 0.2 & 4.8 & 33.4 & 86 & 36.2 & & 368 & 0.36 \\
\hline 04161810 & $12-11-2002$ & 0.3 & 3.7 & 35.4 & 41 & 17.6 & & 511 & 0.46 \\
\hline 04161810 & $01-28-2003$ & 0.5 & 6.0 & 46.0 & 73 & 33 & & 646 & 0.69 \\
\hline 04161810 & 02-02-2003 & 0.4 & 5.2 & 48.3 & 66 & 60.1 & & 1400 & 0.78 \\
\hline 04161810 & $05-01-2003$ & $<.17$ & 2.5 & 34.2 & 33 & 59.7 & & 473 & 0.57 \\
\hline 04161810 & $06-11-2003$ & 0.2 & 3.7 & 39.0 & 36 & 18.9 & & 501 & 0.53 \\
\hline 04161810 & $07-23-2003$ & 0.5 & 6.2 & 45.5 & 38 & 18.1 & & 595 & 0.54 \\
\hline 04161810 & 08-05-2003 & 0.4 & 7.0 & 36.7 & 29 & 25.9 & & 554 & 0.51 \\
\hline 04161810 & 09-09-2003 & 0.6 & 6.0 & 47.3 & 41 & 11.6 & & 564 & 0.55 \\
\hline 04166000 & 09-18-2001 & 0.2 & 8.6 & 54.1 & 39 & 44.9 & & 552 & 0.52 \\
\hline 04166000 & $11-15-2001$ & 0.2 & 7.6 & 63.0 & 22 & 31.2 & & 644 & 0.42 \\
\hline 04166000 & 01-24-2002 & 0.2 & 6.1 & 63.9 & 63 & 51.7 & & 872 & 0.34 \\
\hline 04166000 & $03-12-2002$ & 0.2 & 5.7 & 54.5 & 40 & 71.9 & & 912 & 0.39 \\
\hline 04166000 & 05-01-2002 & 0.2 & 3.5 & 54.4 & 76 & 31.9 & & 692 & -- \\
\hline 04166000 & 06-06-2002 & 0.2 & 7.3 & 48.3 & 62 & 23.3 & & 662 & 0.51 \\
\hline 04166000 & 07-09-2002 & 0.2 & 12.3 & 47.9 & 47 & 54.3 & & 652 & 0.48 \\
\hline 04166000 & 08-28-2002 & 0.3 & 10.5 & 51.2 & 19 & 41 & & 622 & 0.46 \\
\hline 04166000 & 09-03-2002 & 0.3 & 10.2 & 56.4 & 32 & 42.2 & & 715 & 0.53 \\
\hline 04166000 & $12-12-2002$ & 0.3 & 9.2 & 79.0 & 37 & 33 & & 906 & 0.39 \\
\hline 04166000 & $02-02-2003$ & 0.3 & 9.1 & 78.0 & 42 & 51.2 & & 1190 & 0.32 \\
\hline 04166000 & $04-30-2003$ & 0.2 & 0.9 & 74.5 & 51 & 34.3 & & 886 & 0.43 \\
\hline 04166000 & $05-20-2003$ & 0.2 & 4.1 & 66.7 & 36 & 35.9 & & 801 & 0.61 \\
\hline 04166000 & $07-23-2003$ & 0.3 & 9.0 & 51.9 & 69 & 54.3 & & 695 & 0.46 \\
\hline 04166000 & $08-05-2003$ & 0.3 & 10.2 & 56.0 & 29 & 27.1 & & 705 & 0.5 \\
\hline 04166000 & 09-08-2003 & 0.3 & 11.6 & 53.3 & 28 & 29.5 & & 653 & 0.56 \\
\hline
\end{tabular}


Table 9.Results of synoptic stream-water-quality sample analysis in and around Oakland County, Michigan between September 2001 and September 2003- continued

$[\mathrm{mg} / \mathrm{L}$, milligrams per liter; $\mathrm{mg} / \mathrm{L}$, micrograms per liter; $\mathrm{mS} / \mathrm{cm}$, microsiemens per centimeter; wf, filtered water; $\mathrm{mL}$, milliliter]

\begin{tabular}{|c|c|c|c|c|c|c|c|c|}
\hline 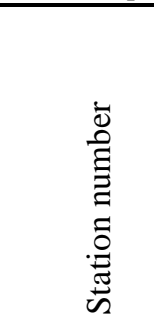 & 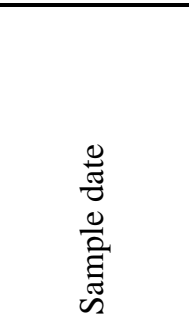 & 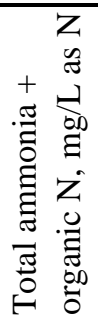 & 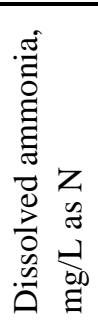 & 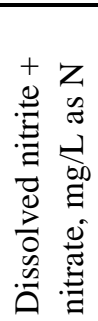 & 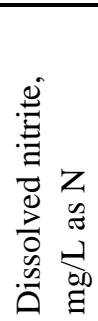 & 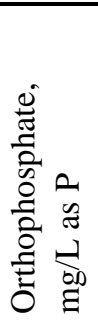 & 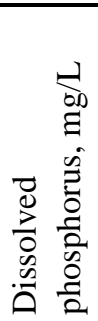 & 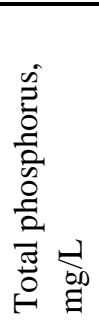 \\
\hline 04161540 & $07-23-2003$ & 0.46 & E.03 & 0.37 & E.006 & $<.02$ & 0.01 & 0.036 \\
\hline 04161540 & 08-05-2003 & 0.55 & $<.04$ & 0.3 & E.006 & $<.02$ & 0.011 & 0.063 \\
\hline 04161540 & 09-09-2003 & 0.25 & $<.04$ & 0.17 & $<.008$ & $<.02$ & 0.007 & 0.02 \\
\hline 04161580 & $12-11-2002$ & 0.41 & E.04 & 0.21 & $<.008$ & $<.02$ & 0.005 & 0.014 \\
\hline 04161580 & $06-11-2003$ & 0.73 & $<.04$ & 0.16 & $<.008$ & $<.02$ & 0.014 & 0.034 \\
\hline 04161580 & 08-05-2003 & 0.31 & $<.04$ & 0.09 & $<.008$ & $<.02$ & 0.013 & 0.022 \\
\hline 04161580 & 09-09-2003 & 0.49 & $<.04$ & 0.11 & $<.008$ & $<.02$ & 0.016 & 0.04 \\
\hline 04161810 & 09-19-2001 & 0.8 & 0.07 & 2.96 & 0.016 & 0.09 & 0.119 & 0.106 \\
\hline 04161810 & $11-15-2001$ & 0.66 & $<.04$ & 1.09 & 0.022 & $<.02$ & 0.028 & 0.004 \\
\hline 04161810 & $01-24-2002$ & 0.51 & E.02 & 1.4 & 0.011 & $<.02$ & 0.009 & $<.004$ \\
\hline 04161810 & $03-12-2002$ & 0.41 & $<.04$ & 1.09 & 0.01 & $<.02$ & 0.008 & E.002 \\
\hline 04161810 & 05-01-2002 & 0.45 & $<.04$ & 0.89 & 0.008 & $<.02$ & 0.009 & $<.004$ \\
\hline 04161810 & 06-06-2002 & 0.71 & 0.07 & 0.77 & 0.018 & E.01 & 0.026 & 0.006 \\
\hline 04161810 & 07-09-2002 & 0.43 & $<.04$ & 3.39 & 0.023 & 0.03 & 0.049 & 0.07 \\
\hline 04161810 & 08-21-2002 & 0.51 & $<.04$ & 3.33 & 0.011 & 0.09 & 0.109 & 0.131 \\
\hline 04161810 & $11-11-2002$ & 0.61 & $<.04$ & 0.56 & E.007 & 0.02 & 0.035 & 0.107 \\
\hline 04161810 & $12-11-2002$ & 0.61 & 0.06 & 1.53 & 0.009 & 0.06 & 0.057 & 0.085 \\
\hline 04161810 & $01-28-2003$ & 0.79 & 0.23 & 3.52 & 0.031 & 0.04 & 0.057 & 0.091 \\
\hline 04161810 & $02-02-2003$ & 0.79 & 0.27 & 3.19 & 0.049 & 0.07 & 0.08 & 0.108 \\
\hline 04161810 & $05-01-2003$ & 2.2 & 0.19 & 1.14 & 0.04 & $<.02$ & 0.015 & 0.45 \\
\hline 04161810 & $06-11-2003$ & 0.78 & 0.04 & 1.11 & 0.02 & 0.02 & 0.035 & 0.113 \\
\hline 04161810 & $07-23-2003$ & 0.61 & E.02 & 2.44 & 0.014 & 0.05 & 0.075 & 0.102 \\
\hline 04161810 & $08-05-2003$ & 0.7 & $<.04$ & 2.07 & 0.018 & 0.06 & 0.086 & 0.145 \\
\hline 04161810 & 09-09-2003 & 0.53 & $<.04$ & 5.23 & 0.011 & 0.15 & 0.174 & 0.183 \\
\hline 04166000 & 09-18-2001 & 0.67 & 0.04 & 0.47 & 0.023 & $<.02$ & 0.016 & 0.015 \\
\hline 04166000 & $11-15-2001$ & 0.65 & $<.04$ & 0.48 & 0.013 & $<.02$ & 0.006 & 0.005 \\
\hline 04166000 & 01-24-2002 & 0.4 & $<.04$ & 0.58 & E.007 & $<.02$ & 0.007 & E.003 \\
\hline 04166000 & 03-12-2002 & 0.45 & E.03 & 0.81 & 0.009 & $<.02$ & 0.006 & E.003 \\
\hline 04166000 & 05-01-2002 & -- & -- & -- & -- & -- & -- & -- \\
\hline 04166000 & 06-06-2002 & 0.64 & 0.08 & 0.47 & 0.028 & $<.02$ & 0.018 & E.003 \\
\hline 04166000 & 07-09-2002 & 0.81 & $<.04$ & 0.17 & E.004 & E.01 & 0.029 & 0.09 \\
\hline 04166000 & 08-28-2002 & 0.72 & 0.05 & 0.17 & $<.008$ & $<.02$ & 0.026 & 0.072 \\
\hline 04166000 & 09-03-2002 & 0.68 & 0.08 & 0.18 & E.005 & E.02 & 0.035 & 0.07 \\
\hline 04166000 & $12-12-2002$ & 0.42 & 0.1 & 0.39 & 0.009 & $<.02$ & 0.008 & 0.021 \\
\hline 04166000 & $02-02-2003$ & 0.45 & 0.09 & 0.43 & 0.011 & $<.02$ & E.004 & 0.03 \\
\hline 04166000 & 04-30-2003 & 0.61 & $<.04$ & 0.16 & E.005 & $<.02$ & 0.011 & 0.033 \\
\hline 04166000 & $05-20-2003$ & 0.8 & 0.06 & 0.47 & 0.027 & $<.02$ & 0.017 & 0.057 \\
\hline 04166000 & $07-23-2003$ & 0.75 & $<.04$ & 0.25 & $<.008$ & E.01 & 0.037 & 0.094 \\
\hline 04166000 & 08-05-2003 & 0.68 & $<.04$ & 0.32 & E.007 & 0.02 & 0.037 & 0.08 \\
\hline 04166000 & 09-08-2003 & 0.7 & 0.04 & 0.22 & E.004 & $<.18$ & 0.041 & 0.072 \\
\hline
\end{tabular}


Table 9.Results of synoptic stream-water-quality sample analysis in and around Oakland County, Michigan between September 2001 and September 2003- continued

[mg/L, milligrams per liter; $\mathrm{mg} / \mathrm{L}$, micrograms per liter; $\mathrm{mS} / \mathrm{cm}$, microsiemens per centimeter; wf, filtered water; $\mathrm{mL}$, milliliter]

\begin{tabular}{|c|c|c|c|c|c|}
\hline 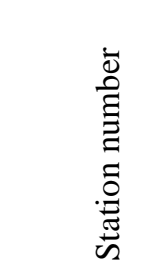 & 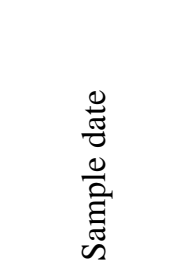 & 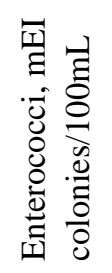 & 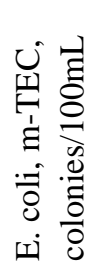 & 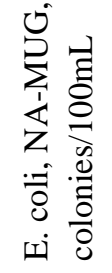 & 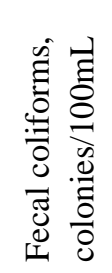 \\
\hline 04161540 & $07-23-2003$ & -- & -- & -- & -- \\
\hline 04161540 & 08-05-2003 & $>1000$ & -- & E1500 & E3500 \\
\hline 04161540 & 09-09-2003 & E350 & -- & E470 & E480 \\
\hline 04161580 & $12-11-2002$ & -- & -- & -- & -- \\
\hline 04161580 & $06-11-2003$ & -- & 110 & -- & -- \\
\hline 04161580 & 08-05-2003 & E100 & -- & E270 & E990 \\
\hline 04161580 & 09-09-2003 & 95 & -- & E260 & E360 \\
\hline 04161810 & 09-19-2001 & -- & -- & -- & -- \\
\hline 04161810 & $11-15-2001$ & -- & -- & -- & -- \\
\hline 04161810 & $01-24-2002$ & -- & -- & -- & -- \\
\hline 04161810 & 03-12-2002 & -- & 120 & -- & -- \\
\hline 04161810 & $05-01-2002$ & -- & -- & -- & -- \\
\hline 04161810 & 06-06-2002 & -- & 490 & -- & -- \\
\hline 04161810 & $07-09-2002$ & -- & 130 & -- & -- \\
\hline 04161810 & $08-21-2002$ & -- & 240 & -- & -- \\
\hline 04161810 & $11-11-2002$ & -- & 3200 & -- & -- \\
\hline 04161810 & $12-11-2002$ & -- & -- & -- & -- \\
\hline 04161810 & $01-28-2003$ & -- & -- & -- & -- \\
\hline 04161810 & 02-02-2003 & -- & 340 & -- & -- \\
\hline 04161810 & $05-01-2003$ & -- & -- & -- & -- \\
\hline 04161810 & 06-11-2003 & -- & 40 & -- & -- \\
\hline 04161810 & $07-23-2003$ & -- & -- & -- & -- \\
\hline 04161810 & 08-05-2003 & E1030 & -- & E1800 & E3400 \\
\hline 04161810 & 09-09-2003 & 69 & -- & E310 & E570 \\
\hline 04166000 & 09-18-2001 & -- & -- & -- & -- \\
\hline 04166000 & $11-15-2001$ & -- & -- & -- & -- \\
\hline 04166000 & $01-24-2002$ & -- & -- & -- & -- \\
\hline 04166000 & $03-12-2002$ & -- & 61 & -- & -- \\
\hline 04166000 & 05-01-2002 & -- & 120 & -- & -- \\
\hline 04166000 & 06-06-2002 & -- & 550 & -- & -- \\
\hline 04166000 & 07-09-2002 & -- & 600 & -- & -- \\
\hline 04166000 & $08-28-2002$ & -- & -- & -- & -- \\
\hline 04166000 & 09-03-2002 & -- & -- & -- & -- \\
\hline 04166000 & $12-12-2002$ & -- & -- & -- & -- \\
\hline 04166000 & 02-02-2003 & -- & 830 & -- & -- \\
\hline 04166000 & $04-30-2003$ & -- & 410 & -- & -- \\
\hline 04166000 & $05-20-2003$ & -- & 800 & -- & -- \\
\hline 04166000 & $07-23-2003$ & -- & -- & -- & -- \\
\hline 04166000 & 08-05-2003 & E850 & -- & E1000 & E2000 \\
\hline 04166000 & 09-08-2003 & E420 & -- & E910 & E910 \\
\hline
\end{tabular}


Table 9.Results of synoptic stream-water-quality sample analysis in and around Oakland County, Michigan between September 2001 and September 2003- continued

[mg/L, milligrams per liter; $\mathrm{mg} / \mathrm{L}$, micrograms per liter; $\mathrm{mS} / \mathrm{cm}$, microsiemens per centimeter; wf, filtered water; $\mathrm{mL}$, milliliter]

\begin{tabular}{|c|c|c|c|c|c|c|c|c|}
\hline 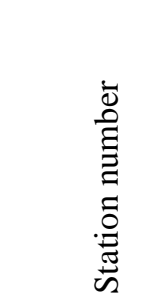 & 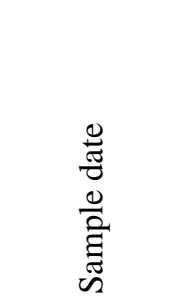 & 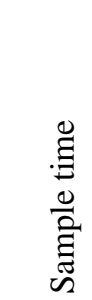 & 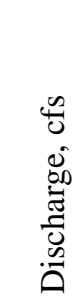 & 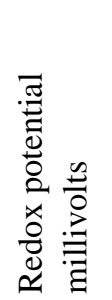 & 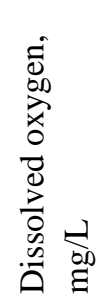 & 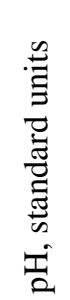 & 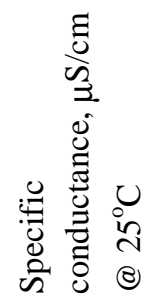 & 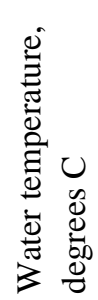 \\
\hline 04166100 & 09-18-2001 & 1215 & 16 & -- & 8.9 & 7.8 & 1100 & 15.7 \\
\hline 04166100 & $11-15-2001$ & 1545 & 200 & -- & 10.1 & 7.7 & 1030 & 10.5 \\
\hline 04166100 & 01-23-2002 & 1200 & 59 & -- & 13.2 & 7.8 & 1820 & 1.8 \\
\hline 04166100 & $03-13-2002$ & 1510 & 131 & -- & 12.6 & 8.8 & 1570 & 6.4 \\
\hline 04166100 & 05-02-2002 & 1245 & 298 & -- & 10.1 & 7.8 & 823 & 10.5 \\
\hline 04166100 & 06-05-2002 & 1245 & 87 & -- & 8.9 & 8.0 & 1080 & 16.8 \\
\hline 04166100 & $07-10-2002$ & 1510 & 15 & -- & 7.2 & 7.9 & 1270 & 22.6 \\
\hline 04166100 & 08-20-2002 & 1400 & -- & -- & 8.1 & 8.2 & 942 & 20.1 \\
\hline 04166100 & $11-11-2002$ & 1430 & 130 & 175 & 8.2 & 7.7 & 805 & 10.8 \\
\hline 04166100 & $12-10-2002$ & 1400 & 26 & 198 & 14.5 & 6.8 & 1720 & -0.1 \\
\hline 04166100 & $01-28-2003$ & 1400 & 29 & -- & 11.7 & 7.7 & 1990 & -0.3 \\
\hline 04166100 & 01-29-2003 & 1600 & 31 & -- & 11.2 & 7.6 & 2770 & -0.2 \\
\hline 04166100 & 02-02-2003 & 1200 & 44 & -- & -- & 7.7 & 6880 & -0.4 \\
\hline 04166100 & 04-30-2003 & 1530 & 32 & -- & -- & 8.1 & 1560 & 13.4 \\
\hline 04166100 & $07-22-2003$ & 1045 & -- & -- & -- & 7.8 & 1420 & -- \\
\hline 04166100 & 08-05-2003 & 1030 & 17 & 345 & 6.8 & 7.8 & 1140 & 20.4 \\
\hline 04166100 & 09-08-2003 & 1045 & 10 & 364 & 8.2 & 8.0 & 1260 & 17.5 \\
\hline 04166200 & $12-10-2002$ & 1445 & 0.64 & 190 & 12.6 & 8.2 & 5200 & 0.0 \\
\hline 04166200 & 08-05-2003 & 1230 & 3.2 & 485 & 4.7 & 7.7 & 664 & 20.6 \\
\hline 04166200 & 09-08-2003 & 1300 & 1.4 & 359 & 6.6 & 7.9 & 2810 & 18.1 \\
\hline 04166315 & 09-18-2001 & 1000 & 5.6 & -- & 9.1 & 7.9 & 1240 & 15.0 \\
\hline 04166315 & $11-19-2001$ & 1045 & 14 & -- & 10.3 & 7.9 & 1010 & 9.6 \\
\hline 04166315 & $01-23-2002$ & 1045 & 11 & -- & 13.3 & 7.8 & 1780 & 1.3 \\
\hline 04166315 & 03-13-2002 & 1330 & 26 & -- & 12.5 & 8.4 & 1780 & 6.0 \\
\hline 04166315 & 05-02-2002 & 1015 & 68 & -- & 10.2 & 8.0 & 1080 & 9.6 \\
\hline 04166315 & 06-05-2002 & 1045 & 18 & -- & -- & 8.1 & 1070 & 16.1 \\
\hline 04166315 & 07-10-2002 & 1340 & 5.1 & -- & 7.5 & 7.0 & 1260 & 21.4 \\
\hline 04166315 & $08-21-2002$ & 815 & 4.9 & 175 & 8.1 & 8.0 & 1140 & 17.7 \\
\hline 04166315 & 11-11-2002 & 1530 & 21 & 169 & 9.4 & 7.8 & 786 & 10.9 \\
\hline 04166315 & $12-10-2002$ & 1230 & -- & 173 & 14.2 & 7.0 & 1570 & -0.2 \\
\hline 04166315 & $01-28-2003$ & 1300 & 3 & -- & 11.8 & 7.5 & 1990 & -0.3 \\
\hline 04166315 & $02-02-2003$ & 1045 & 3.3 & -- & 12.1 & 7.5 & 4540 & -0.3 \\
\hline 04166315 & $04-30-2003$ & 1400 & 11 & -- & 10.3 & 7.9 & 2020 & 12.9 \\
\hline 04166315 & $05-20-2003$ & 1500 & 13 & -- & 8.6 & 8.0 & 1580 & 16.8 \\
\hline 04166315 & $07-22-2003$ & 1230 & 7.3 & -- & -- & 8.1 & 1480 & -- \\
\hline 04166315 & 08-05-2003 & 1115 & 5.9 & 411 & 7.1 & 8.0 & 1260 & 20.1 \\
\hline 04166315 & 09-08-2003 & 1145 & 3.7 & -- & 9 & 8.2 & 1360 & 17.6 \\
\hline 04170000 & 09-17-2001 & 1215 & 32 & -- & 10.4 & 7.8 & 881 & 20.1 \\
\hline 04170000 & 11-19-2001 & 1210 & 165 & -- & 10.8 & 7.7 & 726 & 10.0 \\
\hline 04170000 & $01-23-2002$ & 1420 & 102 & -- & 13.4 & 7.7 & 797 & 2.2 \\
\hline
\end{tabular}


Table 9.Results of synoptic stream-water-quality sample analysis in and around Oakland County, Michigan between September 2001 and September 2003- continued

$[\mathrm{mg} / \mathrm{L}$, milligrams per liter; $\mathrm{mg} / \mathrm{L}$, micrograms per liter; $\mathrm{mS} / \mathrm{cm}$, microsiemens per centimeter; wf, filtered water; $\mathrm{mL}$, milliliter]

\begin{tabular}{|c|c|c|c|c|c|c|c|c|}
\hline 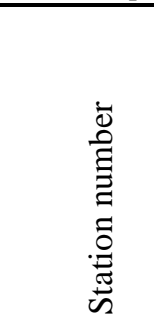 & 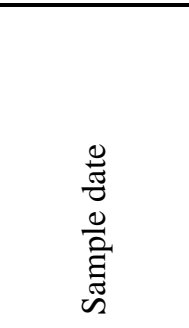 & 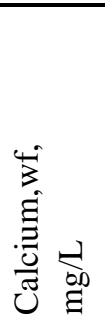 & 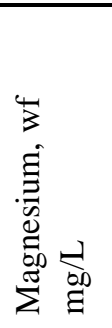 & 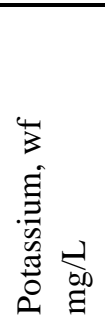 & 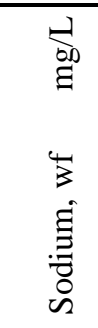 & 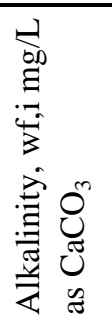 & 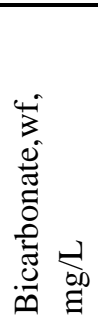 & 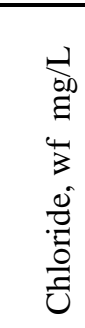 \\
\hline 04166100 & 09-18-2001 & 87.1 & 24.9 & 4.1 & 104 & 188 & 230 & 181 \\
\hline 04166100 & $11-15-2001$ & 84.6 & 21.9 & 3.8 & 94.5 & -- & -- & 173 \\
\hline 04166100 & $01-23-2002$ & 110.0 & 28.4 & 3.7 & 214 & 252 & 308 & 381 \\
\hline 04166100 & 03-13-2002 & 101.0 & 24.0 & 3.6 & 176 & 254 & 310 & 319 \\
\hline 04166100 & 05-02-2002 & 56.3 & 14.9 & 2.7 & 84.2 & 144 & 176 & 150 \\
\hline 04166100 & $06-05-2002$ & 83.6 & 22.7 & 3.6 & 106 & 228 & 278 & 200 \\
\hline 04166100 & 07-10-2002 & 91.9 & 28.7 & 4.0 & 129 & 246 & 300 & 237 \\
\hline 04166100 & 08-20-2002 & 74.3 & 22.3 & 3.9 & 93 & 188 & 229 & 167 \\
\hline 04166100 & $11-11-2002$ & 72.1 & 19.0 & 4.3 & 65.6 & 182 & 222 & 125 \\
\hline 04166100 & $12-10-2002$ & 117.0 & 32.6 & 3.9 & 178 & 273 & 332 & 334 \\
\hline 04166100 & $01-28-2003$ & 134.0 & 37.7 & 3.7 & 245 & 343 & 419 & 411 \\
\hline 04166100 & 01-29-2003 & 135.0 & 38.4 & 3.8 & 436 & 374 & 456 & 681 \\
\hline 04166100 & 02-02-2003 & 137.0 & 36.2 & 7.2 & 1320 & 305 & 372 & 2060 \\
\hline 04166100 & 04-30-2003 & 103.0 & 29.6 & 4.9 & 189 & 216 & 263 & 335 \\
\hline 04166100 & $07-22-2003$ & 79.3 & 21.3 & 5.0 & 179 & -- & -- & 310 \\
\hline 04166100 & 08-05-2003 & 78.9 & 21.8 & 4.5 & 125 & 278 & 339 & 217 \\
\hline 04166100 & 09-08-2003 & 94.4 & 27.9 & 4.6 & 132 & 216 & 263 & 239 \\
\hline 04166200 & $12-10-2002$ & 138.0 & 41.5 & 7.5 & 875 & 287 & -- & 1510 \\
\hline 04166200 & 08-05-2003 & 41.8 & 7.4 & 3.8 & 76.9 & 144 & -- & 134 \\
\hline 04166200 & 09-08-2003 & 188.0 & 37.7 & 11.7 & 539 & 224 & -- & 752 \\
\hline 04166315 & 09-18-2001 & 95.3 & 25.5 & 7.5 & 119 & 205 & 250 & 212 \\
\hline 04166315 & 11-19-2001 & 94.2 & 20.7 & 4.6 & 80.6 & 218 & 266 & 153 \\
\hline 04166315 & $01-23-2002$ & 113.0 & 27.3 & 5.4 & 200 & 251 & 306 & 359 \\
\hline 04166315 & 03-13-2002 & 99.2 & 22.3 & 4.5 & 216 & 254 & 310 & 383 \\
\hline 04166315 & 05-02-2002 & 65.1 & 15.8 & 3.7 & 122 & 156 & 190 & 230 \\
\hline 04166315 & 06-05-2002 & 85.8 & 20.5 & 4.6 & 109 & 232 & 283 & 200 \\
\hline 04166315 & 07-10-2002 & 89.3 & 25.6 & 7.3 & 133 & 230 & 281 & 243 \\
\hline 04166315 & $08-21-2002$ & 91.9 & 25.9 & 7.9 & 114 & 248 & 303 & 212 \\
\hline 04166315 & $11-11-2002$ & 68.6 & 15.5 & 6.9 & 69.4 & 165 & 201 & 124 \\
\hline 04166315 & $12-10-2002$ & 116.0 & 30.0 & 7.3 & 157 & 266 & 325 & 301 \\
\hline 04166315 & $01-28-2003$ & 117.0 & 30.6 & 8.8 & 271 & 262 & 320 & 442 \\
\hline 04166315 & 02-02-2003 & 122.0 & 30.3 & 8.6 & 795 & 262 & 320 & 1290 \\
\hline 04166315 & 04-30-2003 & 118.0 & 30.5 & 7.8 & 258 & 230 & 281 & 469 \\
\hline 04166315 & $05-20-2003$ & 95.3 & 21.9 & 7.5 & 178 & 208 & 254 & 343 \\
\hline 04166315 & $07-22-2003$ & 94.3 & 25.4 & 9.0 & 170 & -- & -- & 315 \\
\hline 04166315 & 08-05-2003 & 81.6 & 19.9 & 7.8 & 145 & 260 & -- & 255 \\
\hline 04166315 & 09-08-2003 & 90.7 & 24.9 & 9.4 & 142 & 176 & 215 & 267 \\
\hline 04170000 & 09-17-2001 & 63.0 & 22.3 & 4.0 & 81.6 & 218 & 266 & 141 \\
\hline 04170000 & 11-19-2001 & 66.5 & 19.2 & 2.8 & 52.2 & 196 & 239 & 99 \\
\hline 04170000 & 01-23-2002 & 69.5 & 20.5 & 2.9 & 62.7 & 192 & 234 & 111 \\
\hline
\end{tabular}


Table 9.Results of synoptic stream-water-quality sample analysis in and around Oakland County, Michigan between September 2001 and September 2003- continued

[mg/L, milligrams per liter; $\mathrm{mg} / \mathrm{L}$, micrograms per liter; $\mathrm{mS} / \mathrm{cm}$, microsiemens per centimeter; wf, filtered water; $\mathrm{mL}$, milliliter]

\begin{tabular}{|c|c|c|c|c|c|c|c|c|c|}
\hline 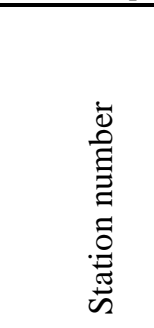 & 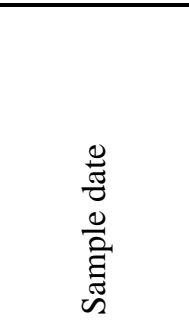 & 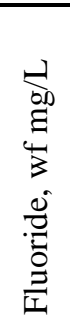 & 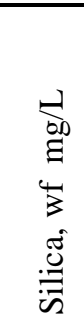 & 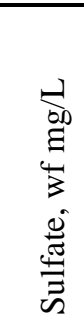 & 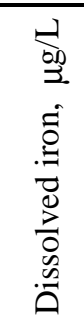 & 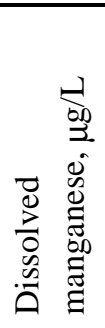 & $\begin{array}{l} \\
+ \\
0 \\
0 \\
\frac{0}{0} \\
\simeq \\
\simeq\end{array}$ & 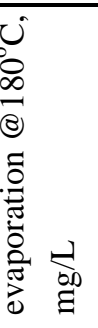 & 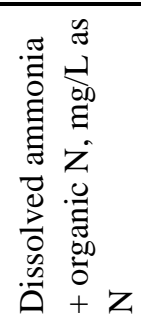 \\
\hline 04166100 & 09-18-2001 & 0.3 & 10.1 & 55.9 & 11 & 29 & & 624 & 0.45 \\
\hline 04166100 & $11-15-2001$ & 0.2 & 6.7 & 52.4 & 27 & 38.6 & & 604 & 0.32 \\
\hline 04166100 & $01-23-2002$ & 0.4 & 6.5 & 57.6 & 49 & 64.8 & & 1010 & 0.28 \\
\hline 04166100 & 03-13-2002 & 0.2 & 5.3 & 55.5 & 48 & 81.6 & & 880 & 0.33 \\
\hline 04166100 & 05-02-2002 & 0.1 & 2.8 & 33.4 & 40 & 45.2 & & 465 & 0.55 \\
\hline 04166100 & 06-05-2002 & 0.2 & 7.5 & 47.3 & 48 & 31.1 & & 652 & 0.52 \\
\hline 04166100 & 07-10-2002 & 0.4 & 13.0 & 55.2 & 13 & 43.6 & & 753 & 0.43 \\
\hline 04166100 & $08-20-2002$ & 0.4 & 10.6 & 47.7 & 13 & 37.5 & & 592 & 0.39 \\
\hline 04166100 & $11-11-2002$ & 0.2 & 7.4 & 45.5 & 82 & 49.5 & & 476 & 0.45 \\
\hline 04166100 & $12-10-2002$ & 0.4 & 9.3 & 69.0 & 53 & 51.2 & & 1000 & 0.25 \\
\hline 04166100 & $01-28-2003$ & 0.4 & 9.7 & 73.7 & 83 & 81.8 & & 1140 & 0.21 \\
\hline 04166100 & 01-29-2003 & 0.3 & 10.1 & 73.7 & 129 & 87.9 & & 1570 & 0.24 \\
\hline 04166100 & 02-02-2003 & 0.3 & 9.4 & 84.1 & 66 & 174 & & 3880 & 0.44 \\
\hline 04166100 & 04-30-2003 & 0.3 & 1.9 & 68.5 & 120 & 103 & & 903 & 0.39 \\
\hline 04166100 & $07-22-2003$ & 0.3 & 9.2 & 48.9 & 30 & 49.8 & & 822 & 0.51 \\
\hline 04166100 & 08-05-2003 & 0.4 & 9.6 & 46.7 & 21 & 50.1 & & 688 & 0.48 \\
\hline 04166100 & 09-08-2003 & 0.4 & 10.7 & 55.2 & 16 & 38.3 & & 738 & 0.4 \\
\hline 04166200 & $12-10-2002$ & 0.5 & 7.6 & 78.4 & 30 & 189 & & 3020 & 0.44 \\
\hline 04166200 & 08-05-2003 & 0.2 & 4.6 & 30.6 & 23 & 80.7 & & 391 & -- \\
\hline 04166200 & 09-08-2003 & 0.6 & 10.8 & 71.6 & 24 & 65.6 & & 1670 & 0.9 \\
\hline 04166315 & 09-18-2001 & 0.4 & 9.9 & 54.7 & 10 & 14 & & 708 & 0.36 \\
\hline 04166315 & 11-19-2001 & 0.4 & 6.7 & 52.0 & 38 & 29.9 & & 612 & 0.32 \\
\hline 04166315 & $01-23-2002$ & 0.4 & 6.7 & 54.9 & 64 & 50.4 & & 988 & 0.38 \\
\hline 04166315 & 03-13-2002 & 0.3 & 4.5 & 49.9 & 62 & 66.3 & & 976 & 0.36 \\
\hline 04166315 & 05-02-2002 & 0.2 & 3.1 & 34.3 & 46 & 33.7 & & 618 & 0.56 \\
\hline 04166315 & 06-05-2002 & 0.3 & 7.9 & 36.7 & 74 & 27.2 & & 663 & 0.52 \\
\hline 04166315 & 07-10-2002 & 0.5 & 12.2 & 52.3 & 21 & 28 & & 754 & 0.47 \\
\hline 04166315 & $08-21-2002$ & 0.4 & 12.1 & 49.5 & 16 & 18.8 & & 768 & 0.38 \\
\hline 04166315 & $11-11-2002$ & 0.3 & 7.3 & 46.2 & 77 & 25.2 & & 463 & 0.44 \\
\hline 04166315 & $12-10-2002$ & 0.5 & 10.5 & 62.2 & 52 & 21.7 & & 905 & 0.43 \\
\hline 04166315 & $01-28-2003$ & 0.4 & 9.9 & 56.1 & 62 & 39.3 & & 1130 & 0.5 \\
\hline 04166315 & 02-02-2003 & 0.4 & 10.4 & 63.1 & 70 & 72.3 & & 2510 & 0.6 \\
\hline 04166315 & 04-30-2003 & 0.4 & 3.9 & 63.0 & 90 & 63.9 & & 1130 & 0.51 \\
\hline 04166315 & $05-20-2003$ & 0.3 & 4.0 & 52.3 & 80 & 55.7 & & 900 & 0.71 \\
\hline 04166315 & $07-22-2003$ & 0.4 & 10.0 & 52.9 & 21 & 17.5 & & 863 & 0.65 \\
\hline 04166315 & 08-05-2003 & 0.4 & 9.5 & 45.0 & 20 & 17.9 & & 733 & 0.46 \\
\hline 04166315 & 09-08-2003 & 0.5 & 10.6 & 46.4 & 19 & 13.5 & & 785 & 0.36 \\
\hline 04170000 & 09-17-2001 & 0.3 & 10.3 & 33.4 & 44 & 16.4 & & 495 & 0.38 \\
\hline 04170000 & 11-19-2001 & 0.2 & 6.8 & 31.0 & 34 & 19.7 & & 438 & 0.39 \\
\hline 04170000 & 01-23-2002 & 0.3 & 6.8 & 31.2 & 34 & 25.3 & & 460 & 0.35 \\
\hline
\end{tabular}


Table 9.Results of synoptic stream-water-quality sample analysis in and around Oakland County, Michigan between September 2001 and September 2003- continued

$[\mathrm{mg} / \mathrm{L}$, milligrams per liter; $\mathrm{mg} / \mathrm{L}$, micrograms per liter; $\mathrm{mS} / \mathrm{cm}$, microsiemens per centimeter; wf, filtered water; $\mathrm{mL}$, milliliter]

\begin{tabular}{|c|c|c|c|c|c|c|c|c|}
\hline 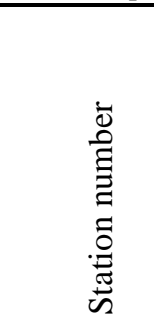 & 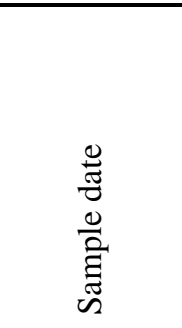 & 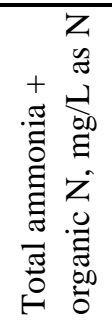 & 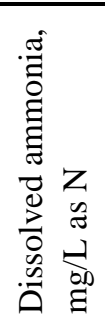 & 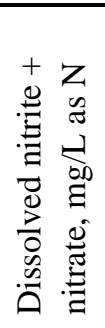 & 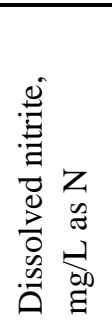 & 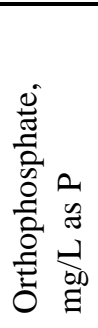 & 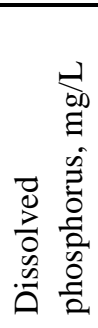 & 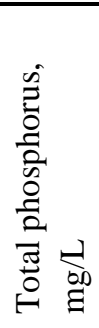 \\
\hline 04166100 & 09-18-2001 & 0.5 & $<<.04$ & 0.5 & 0.006 & E.01 & 0.026 & 0.025 \\
\hline 04166100 & $11-15-2001$ & 0.49 & $<.04$ & 0.47 & 0.013 & $<.02$ & 0.01 & 0.004 \\
\hline 04166100 & $01-23-2002$ & 0.37 & E.02 & 0.61 & E.007 & $<.02$ & 0.007 & $<.004$ \\
\hline 04166100 & 03-13-2002 & 0.32 & $<.04$ & 0.73 & 0.009 & $<.02$ & 0.007 & 0.004 \\
\hline 04166100 & 05-02-2002 & 1.2 & 0.09 & 0.48 & 0.015 & $<.04$ & 0.013 & 0.2 \\
\hline 04166100 & $06-05-2002$ & 0.74 & 0.08 & 0.48 & 0.026 & $<.02$ & 0.019 & 0.006 \\
\hline 04166100 & $07-10-2002$ & 0.79 & 0.04 & 0.42 & 0.012 & 0.03 & 0.045 & 0.12 \\
\hline 04166100 & $08-20-2002$ & 0.59 & 0.04 & 0.49 & 0.009 & 0.03 & 0.039 & 0.113 \\
\hline 04166100 & $11-11-2002$ & 0.83 & 0.1 & 0.33 & 0.016 & $<.02$ & 0.007 & 0.132 \\
\hline 04166100 & $12-10-2002$ & 0.28 & $<.04$ & 0.37 & 0.008 & $<.02$ & 0.006 & 0.088 \\
\hline 04166100 & $01-28-2003$ & 0.27 & E.03 & 0.39 & E.007 & $<.02$ & 0.008 & 0.027 \\
\hline 04166100 & 01-29-2003 & 0.26 & E.03 & 0.39 & 0.008 & $<.02$ & 0.009 & 0.021 \\
\hline 04166100 & 02-02-2003 & 0.52 & 0.19 & 0.51 & 0.028 & $<.02$ & 0.008 & 0.029 \\
\hline 04166100 & 04-30-2003 & 0.6 & $<.04$ & 0.11 & E.004 & $<.02$ & 0.014 & 0.049 \\
\hline 04166100 & $07-22-2003$ & 1 & 0.07 & 0.54 & 0.021 & E.01 & 0.029 & 0.098 \\
\hline 04166100 & 08-05-2003 & 0.67 & 0.06 & 0.29 & 0.008 & 0.02 & 0.036 & 0.097 \\
\hline 04166100 & 09-08-2003 & 0.54 & E.04 & 0.24 & E.004 & $<.18$ & 0.03 & 0.083 \\
\hline 04166200 & $12-10-2002$ & 0.46 & 0.07 & 1.11 & 0.038 & E.01 & 0.018 & 0.061 \\
\hline 04166200 & 08-05-2003 & -- & -- & -- & -- & -- & -- & -- \\
\hline 04166200 & 09-08-2003 & 0.94 & $<.20$ & 0.9 & E.022 & $<.18$ & 0.062 & 0.099 \\
\hline 04166315 & 09-18-2001 & 0.4 & $<.04$ & 1.67 & E.004 & E.01 & 0.019 & 0.01 \\
\hline 04166315 & $11-19-2001$ & 0.42 & $<.04$ & 1.41 & E.005 & $<.02$ & 0.007 & 0.006 \\
\hline 04166315 & $01-23-2002$ & 0.45 & $<.04$ & 2.97 & 0.01 & $<.02$ & 0.009 & $<.004$ \\
\hline 04166315 & 03-13-2002 & 0.33 & $<.04$ & 1.19 & E.006 & $<.02$ & 0.015 & 0.005 \\
\hline 04166315 & 05-02-2002 & 1.3 & 0.06 & 0.98 & 0.021 & $<.04$ & 0.01 & 0.19 \\
\hline 04166315 & 06-05-2002 & 0.62 & E.04 & 1.38 & 0.023 & $<.02$ & 0.019 & E.002 \\
\hline 04166315 & 07-10-2002 & 0.72 & E.02 & 1.67 & 0.012 & 0.03 & 0.032 & 0.08 \\
\hline 04166315 & $08-21-2002$ & 0.44 & $<.04$ & 1.11 & $<.008$ & 0.02 & 0.029 & 0.054 \\
\hline 04166315 & $11-11-2002$ & 0.63 & $<.04$ & 1.52 & E.007 & $<.02$ & 0.01 & 0.057 \\
\hline 04166315 & $12-10-2002$ & 0.48 & E.03 & 5.02 & 0.022 & $<.02$ & 0.008 & 0.016 \\
\hline 04166315 & $01-28-2003$ & 0.52 & 0.07 & 7.1 & 0.045 & $<.02$ & 0.015 & 0.03 \\
\hline 04166315 & $02-02-2003$ & 0.63 & 0.25 & 4.69 & 0.053 & $<.02$ & 0.01 & 0.025 \\
\hline 04166315 & 04-30-2003 & 0.67 & $<.04$ & 1.08 & 0.011 & $<.02$ & 0.014 & 0.033 \\
\hline 04166315 & $05-20-2003$ & 0.92 & 0.08 & 1.71 & 0.036 & $<.02$ & 0.017 & 0.064 \\
\hline 04166315 & $07-22-2003$ & 0.8 & E.03 & 2.26 & 0.011 & E.01 & 0.021 & 0.039 \\
\hline 04166315 & 08-05-2003 & 0.52 & $<.04$ & 2.46 & 0.01 & E.01 & 0.021 & 0.039 \\
\hline 04166315 & 09-08-2003 & 0.45 & $<.04$ & 3.1 & E.005 & $<.18$ & 0.023 & 0.038 \\
\hline 04170000 & 09-17-2001 & 0.44 & $<.04$ & 0.76 & 0.011 & $<.02$ & 0.009 & 0.017 \\
\hline 04170000 & $11-19-2001$ & 0.6 & 0.05 & 0.3 & 0.008 & $<.02$ & 0.007 & 0.006 \\
\hline 04170000 & 01-23-2002 & 0.4 & 0.05 & 0.4 & 0.011 & $<.02$ & 0.004 & $<.004$ \\
\hline
\end{tabular}


Table 9.Results of synoptic stream-water-quality sample analysis in and around Oakland County, Michigan between September 2001 and September 2003- continued

[mg/L, milligrams per liter; $\mathrm{mg} / \mathrm{L}$, micrograms per liter; $\mathrm{mS} / \mathrm{cm}$, microsiemens per centimeter; wf, filtered water; $\mathrm{mL}$, milliliter]
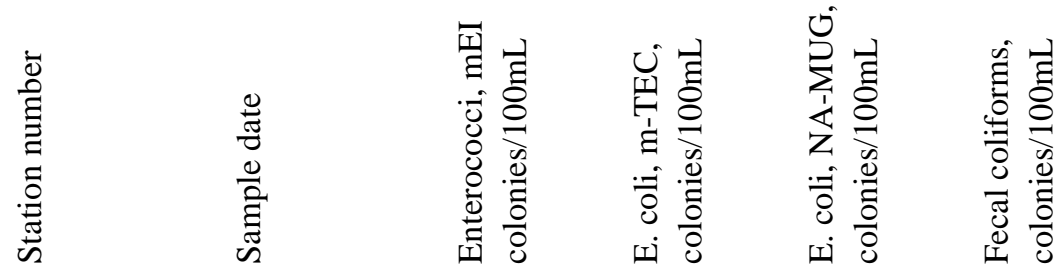

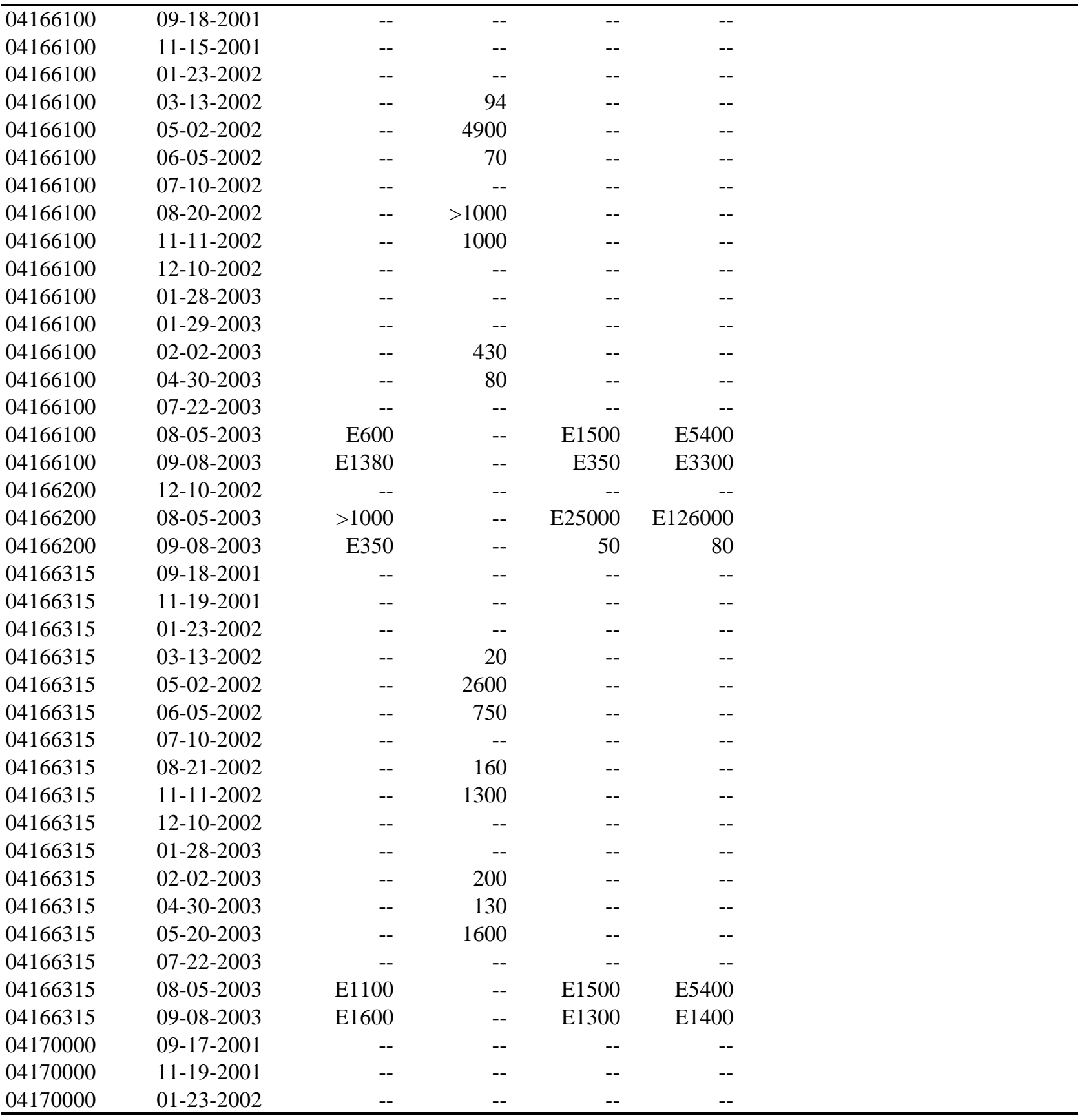


Table 9.Results of synoptic stream-water-quality sample analysis in and around Oakland County, Michigan between September 2001 and September 2003- continued

[mg/L, milligrams per liter; $\mathrm{mg} / \mathrm{L}$, micrograms per liter; $\mathrm{mS} / \mathrm{cm}$, microsiemens per centimeter; wf, filtered water; $\mathrm{mL}$, milliliter]

\begin{tabular}{|c|c|c|c|c|c|c|c|c|}
\hline 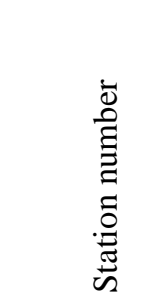 & 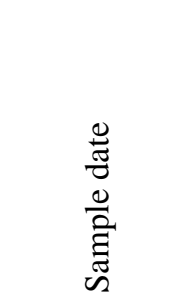 & 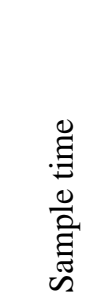 & 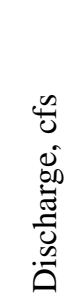 & 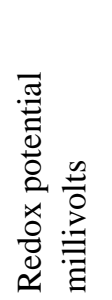 & 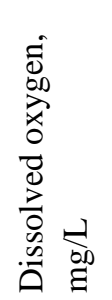 & 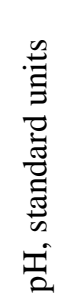 & 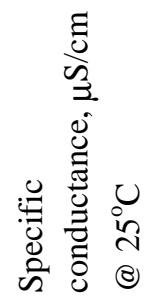 & 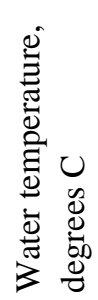 \\
\hline 04170000 & $03-13-2002$ & 1130 & 193 & -- & 12.7 & 8.1 & 796 & 4.9 \\
\hline 04170000 & $05-02-2002$ & 845 & 116 & -- & 11.1 & 8.2 & 763 & 11.0 \\
\hline 04170000 & $06-05-2002$ & 910 & 78 & -- & 7.6 & 8.2 & 749 & 19.2 \\
\hline 04170000 & $07-08-2002$ & 1425 & 32 & -- & -- & 8.2 & 775 & 26.4 \\
\hline 04170000 & $08-20-2002$ & 1200 & -- & -- & 8.9 & 7.8 & 770 & 24.1 \\
\hline 04170000 & 11-11-2002 & 1715 & 86 & 169 & 10.7 & 7.7 & 784 & 8.6 \\
\hline 04170000 & $12-10-2002$ & 1015 & 54 & -- & 14.3 & 8.0 & 847 & 1.6 \\
\hline 04170000 & 02-02-2003 & 915 & 44 & -- & 13.8 & 7.7 & 888 & 0.9 \\
\hline 04170000 & $04-22-2003$ & 1700 & 88 & -- & 7.4 & 7.9 & 832 & 11.9 \\
\hline 04170000 & 05-01-2003 & 900 & 85 & -- & 8.9 & 7.7 & 780 & 15.0 \\
\hline 04170000 & $06-12-2003$ & 1845 & 93 & -- & 8.5 & 7.7 & 791 & 18.4 \\
\hline 04170000 & $07-22-2003$ & 945 & -- & -- & -- & 8.0 & 767 & -- \\
\hline 04170000 & 08-05-2003 & 915 & 29 & 302 & 6.1 & 7.7 & 788 & 21.9 \\
\hline 04170000 & 09-08-2003 & 945 & 20 & 297 & 6.5 & 7.7 & 764 & 19.6 \\
\hline 04170500 & 09-18-2001 & 815 & 483 & -- & 8.4 & 7.8 & 719 & 20.3 \\
\hline 04170500 & $11-19-2001$ & 900 & 201 & -- & 10.8 & 7.7 & 711 & 9.6 \\
\hline 04170500 & 01-23-2002 & 900 & 131 & -- & 13.3 & 7.5 & 788 & 2.3 \\
\hline 04170500 & 03-13-2002 & 910 & 224 & -- & 12.6 & 8.5 & 813 & 2.8 \\
\hline 04170500 & 05-02-2002 & 720 & 121 & -- & 10 & 8.3 & 735 & 11.6 \\
\hline 04170500 & 06-05-2002 & 745 & 75 & -- & 8.9 & 8.0 & 691 & 18.0 \\
\hline 04170500 & 07-08-2002 & 1535 & -- & -- & -- & 8.3 & 682 & -- \\
\hline 04170500 & 08-28-2002 & 800 & 33 & 152 & 7.7 & 8.0 & 655 & 24.2 \\
\hline 04170500 & $12-10-2002$ & 830 & 64 & 164 & 14 & 7.8 & 767 & 1.8 \\
\hline 04170500 & 02-02-2003 & 830 & 58 & -- & 15.6 & 7.6 & 901 & 2.8 \\
\hline 04170500 & 05-01-2003 & 800 & 71 & -- & 10.1 & 8.0 & 732 & 14.1 \\
\hline 04170500 & $07-22-2003$ & 830 & -- & -- & -- & 8.2 & 699 & -- \\
\hline 04170500 & $08-05-2003$ & 815 & 31 & 271 & 7.2 & 8.0 & 705 & 24.5 \\
\hline 04170500 & 09-08-2003 & 845 & 23 & 266 & 8.5 & 8.0 & 668 & 22.6 \\
\hline
\end{tabular}


Table 9.Results of synoptic stream-water-quality sample analysis in and around Oakland County, Michigan between September 2001 and September 2003- continued

[mg/L, milligrams per liter; $\mathrm{mg} / \mathrm{L}$, micrograms per liter; $\mathrm{mS} / \mathrm{cm}$, microsiemens per centimeter; wf, filtered water; $\mathrm{mL}$, milliliter]

\begin{tabular}{|c|c|c|c|c|c|c|c|c|}
\hline 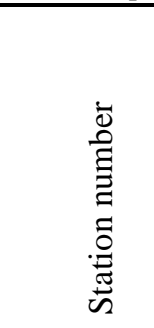 & 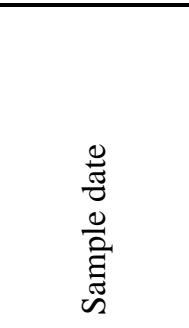 & 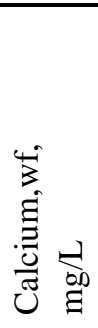 & 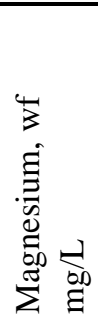 & 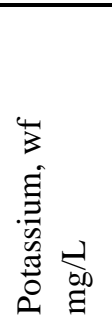 & 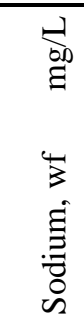 & 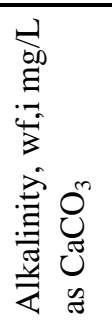 & 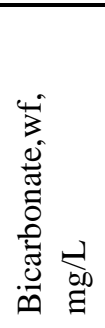 & 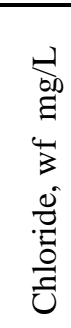 \\
\hline 04170000 & $03-13-2002$ & 64.5 & 18.3 & 2.6 & 64.4 & 194 & 237 & 119 \\
\hline 04170000 & $05-02-2002$ & 66.8 & 19.6 & 2.6 & 58.3 & 260 & 317 & 108 \\
\hline 04170000 & 06-05-2002 & 67.2 & 19.9 & 2.9 & 59.3 & 206 & 251 & 107 \\
\hline 04170000 & 07-08-2002 & 59.5 & 21.5 & 3.3 & 64.6 & 190 & 232 & 119 \\
\hline 04170000 & 08-20-2002 & 59.1 & 22.0 & 4.7 & 67.4 & 168 & 205 & 121 \\
\hline 04170000 & $11-11-2002$ & 70.9 & 22.7 & 3.6 & 60.5 & 201 & 245 & 113 \\
\hline 04170000 & $12-10-2002$ & 73.2 & 22.9 & 3.5 & 64.2 & 196 & 239 & 125 \\
\hline 04170000 & $02-02-2003$ & 76.9 & 23.2 & 3.6 & 70 & 238 & 290 & 131 \\
\hline 04170000 & $04-22-2003$ & 74.1 & 20.6 & 3.4 & 70.2 & 202 & 246 & 128 \\
\hline 04170000 & 05-01-2003 & 74.7 & 22.0 & 3.1 & 64.8 & 190 & 232 & 120 \\
\hline 04170000 & $06-12-2003$ & 72.1 & 22.5 & 3.3 & 67.6 & 192 & 234 & 121 \\
\hline 04170000 & $07-22-2003$ & 61.2 & 22.2 & 4.2 & 66.4 & -- & -- & 124 \\
\hline 04170000 & 08-05-2003 & 64.4 & 23.0 & 4.0 & 64.9 & 266 & 325 & 120 \\
\hline 04170000 & 09-08-2003 & 61.9 & 20.3 & 4.4 & 66.2 & 166 & 203 & 124 \\
\hline 04170500 & 09-18-2001 & 40.0 & 21.0 & 3.2 & 68.1 & 106 & 129 & 120 \\
\hline 04170500 & 11-19-2001 & 63.8 & 19.3 & 2.8 & 50.3 & 168 & 205 & 97 \\
\hline 04170500 & 01-23-2002 & 69.9 & 20.7 & 2.8 & 59.7 & 222 & 271 & 110 \\
\hline 04170500 & 03-13-2002 & 62.6 & 18.6 & 2.5 & 69.5 & 190 & 232 & 126 \\
\hline 04170500 & 05-02-2002 & 60.9 & 18.8 & 2.5 & 55.9 & 180 & 220 & 107 \\
\hline 04170500 & 06-05-2002 & 59.6 & 19.7 & 2.4 & 54.7 & 174 & 212 & 103 \\
\hline 04170500 & 07-08-2002 & 46.2 & 21.1 & 2.3 & 59.8 & -- & -- & 106 \\
\hline 04170500 & 08-28-2002 & 41.0 & 22.0 & 2.7 & 62.7 & 125 & 153 & 113 \\
\hline 04170500 & $12-10-2002$ & 65.5 & 23.7 & 3.3 & 55.7 & 204 & 246 & 110 \\
\hline 04170500 & 02-02-2003 & 79.8 & 25.5 & 3.4 & 71.2 & 246 & 300 & 132 \\
\hline 04170500 & 05-01-2003 & 64.4 & 19.6 & 2.9 & 67.9 & 168 & 205 & 124 \\
\hline 04170500 & $07-22-2003$ & 48.1 & 20.9 & 3.0 & 64.8 & -- & -- & 122 \\
\hline 04170500 & $08-05-2003$ & 46.4 & 20.3 & 3.0 & 67.2 & 144 & 176 & 124 \\
\hline 04170500 & $09-08-2003$ & 40.7 & 22.6 & 3.1 & 65.5 & 116 & 133 & 124 \\
\hline
\end{tabular}


Table 9.Results of synoptic stream-water-quality sample analysis in and around Oakland County, Michigan between September 2001 and September 2003- continued

$[\mathrm{mg} / \mathrm{L}$, milligrams per liter; $\mathrm{mg} / \mathrm{L}$, micrograms per liter; $\mathrm{mS} / \mathrm{cm}$, microsiemens per centimeter; wf, filtered water; $\mathrm{mL}$, milliliter]

\begin{tabular}{|c|c|c|c|c|c|c|c|c|c|}
\hline 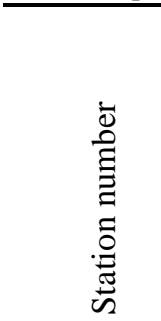 & 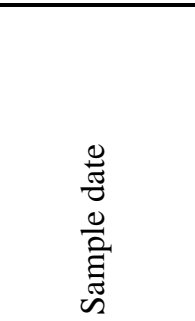 & 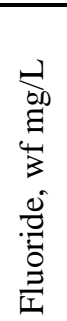 & 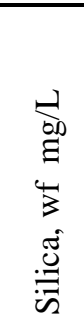 & 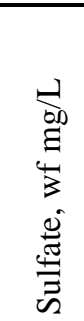 & $\begin{array}{l}\frac{1}{00} \\
3 \\
0 \\
0 \\
.0 \\
0 \\
0 \\
0 \\
0 \\
0 \\
0 \\
0\end{array}$ & 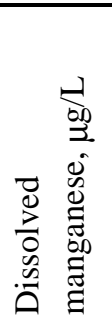 & 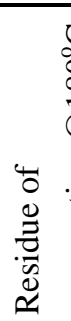 & 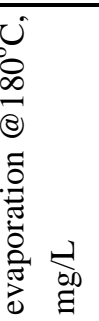 & 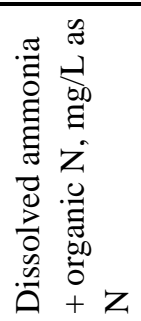 \\
\hline 04170000 & $03-13-2002$ & 0.2 & 5.8 & 28.7 & 40 & 15.4 & & 450 & 0.35 \\
\hline 04170000 & 05-02-2002 & 0.2 & 4.7 & 30.4 & 55 & 12.6 & & 446 & 0.41 \\
\hline 04170000 & 06-05-2002 & 0.2 & 5.9 & 30.1 & 98 & 21.2 & & 466 & 0.43 \\
\hline 04170000 & 07-08-2002 & 0.5 & 5.6 & 32.5 & 95 & 17.1 & & 421 & 0.42 \\
\hline 04170000 & 08-20-2002 & 0.4 & 10.9 & 31.8 & 66 & 30 & & 463 & 0.36 \\
\hline 04170000 & $11-11-2002$ & 0.3 & 8.3 & 35.1 & 34 & 20.8 & & 457 & 0.39 \\
\hline 04170000 & $12-10-2002$ & 0.3 & 8.8 & 35.4 & 32 & 25.7 & & 500 & 0.39 \\
\hline 04170000 & 02-02-2003 & 0.3 & 9.1 & 35.3 & 35 & 37 & & 512 & 0.39 \\
\hline 04170000 & $04-22-2003$ & 0.2 & 5.7 & 41.2 & 49 & 21.5 & & 495 & 0.46 \\
\hline 04170000 & 05-01-2003 & 0.3 & 4.8 & 40.0 & 47 & 16.6 & & 471 & 0.36 \\
\hline 04170000 & $06-12-2003$ & 0.3 & 4.6 & 34.1 & 67 & 18.2 & & 459 & 0.48 \\
\hline 04170000 & $07-22-2003$ & 0.7 & 8.1 & 30.5 & 92 & 28.5 & & 478 & 0.47 \\
\hline 04170000 & 08-05-2003 & 0.4 & 8.9 & 30.2 & 65 & 28.4 & & 486 & 0.42 \\
\hline 04170000 & 09-08-2003 & 0.4 & 9.3 & 33.1 & 59 & 34.1 & & 466 & 0.36 \\
\hline 04170500 & 09-18-2001 & 0.2 & 6.1 & 28.7 & 18 & 4.1 & & 395 & 0.44 \\
\hline 04170500 & 11-19-2001 & 0.2 & 6.1 & 31.2 & 22 & 4.1 & & 442 & 0.39 \\
\hline 04170500 & $01-23-2002$ & 0.3 & 5.6 & 30.9 & 25 & 7.7 & & 454 & 0.34 \\
\hline 04170500 & 03-13-2002 & 0.2 & 4.3 & 29.6 & 32 & 4.8 & & 458 & 0.32 \\
\hline 04170500 & 05-02-2002 & 0.2 & 2.8 & 30.6 & 23 & 4.7 & & 432 & 0.39 \\
\hline 04170500 & 06-05-2002 & 0.2 & 1.4 & 30.8 & 34 & 9.6 & & 430 & 0.44 \\
\hline 04170500 & 07-08-2002 & 0.2 & 2.4 & 28.6 & 47 & 11.6 & & 387 & 0.51 \\
\hline 04170500 & $08-28-2002$ & 0.3 & 5.7 & 25.9 & 17 & E2.7 & & 364 & 0.43 \\
\hline 04170500 & $12-10-2002$ & 0.3 & 3.5 & 35.9 & 24 & 4.3 & & 447 & 0.38 \\
\hline 04170500 & 02-02-2003 & 0.3 & 4.9 & 39.3 & 24 & 5.3 & & 519 & 0.33 \\
\hline 04170500 & 05-01-2003 & 0.2 & 1.9 & 37.5 & 18 & 3.7 & & 435 & 0.34 \\
\hline 04170500 & $07-22-2003$ & 0.2 & 2.6 & 31.1 & 27 & 8.8 & & 443 & 0.47 \\
\hline 04170500 & 08-05-2003 & 0.2 & 3.7 & 31.0 & 29 & 10 & & 468 & 0.54 \\
\hline 04170500 & 09-08-2003 & 0.3 & 5.7 & 27.6 & 28 & 9.3 & & 401 & 0.42 \\
\hline
\end{tabular}


Table 9.Results of synoptic stream-water-quality sample analysis in and around Oakland County, Michigan between September 2001 and September 2003- continued

$[\mathrm{mg} / \mathrm{L}$, milligrams per liter; $\mathrm{mg} / \mathrm{L}$, micrograms per liter; $\mathrm{mS} / \mathrm{cm}$, microsiemens per centimeter; wf, filtered water; $\mathrm{mL}$, milliliter]

\begin{tabular}{|c|c|c|c|c|c|c|c|c|}
\hline 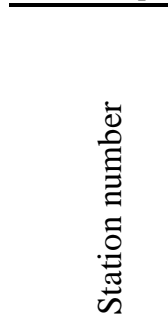 & 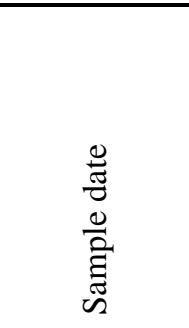 & 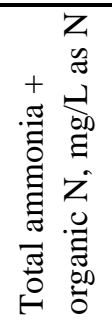 & 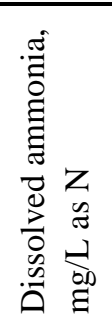 & 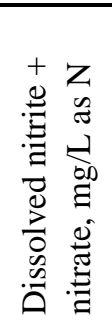 & 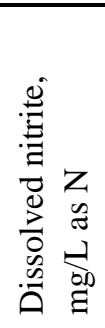 & 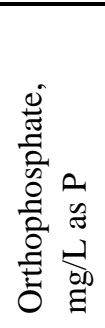 & 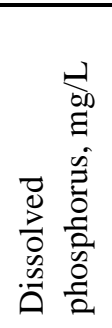 & 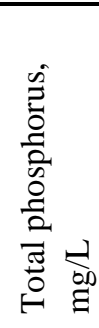 \\
\hline 04170000 & 03-13-2002 & 0.33 & $<.04$ & 0.4 & E.005 & $<.02$ & 0.004 & E.004 \\
\hline 04170000 & $05-02-2002$ & 0.41 & E. 02 & 0.17 & $<.008$ & $<.04$ & 0.007 & E.03 \\
\hline 04170000 & 06-05-2002 & 0.56 & 0.04 & 0.21 & E.005 & $<.02$ & 0.013 & $<.004$ \\
\hline 04170000 & 07-08-2002 & 0.55 & $<.04$ & 0.16 & E.004 & $<.02$ & 0.016 & E.05 \\
\hline 04170000 & 08-20-2002 & 0.51 & E.03 & 0.26 & E.004 & $<.02$ & 0.017 & 0.06 \\
\hline 04170000 & $11-11-2002$ & 0.55 & 0.1 & 0.2 & 0.044 & $<.02$ & 0.007 & 0.032 \\
\hline 04170000 & $12-10-2002$ & 0.4 & 0.08 & 0.32 & 0.07 & $<.02$ & 0.008 & 0.02 \\
\hline 04170000 & $02-02-2003$ & 0.44 & 0.1 & 1.02 & 0.039 & $<.02$ & 0.005 & 0.018 \\
\hline 04170000 & $04-22-2003$ & 0.58 & 0.07 & 0.36 & E.007 & $<.02$ & 0.006 & 0.031 \\
\hline 04170000 & 05-01-2003 & 0.58 & E.03 & 0.24 & E.004 & $<.02$ & 0.006 & 0.03 \\
\hline 04170000 & $06-12-2003$ & 0.67 & 0.06 & 0.47 & 0.015 & $<.02$ & 0.009 & 0.037 \\
\hline 04170000 & $07-22-2003$ & 0.65 & 0.07 & 0.64 & 0.026 & $<.02$ & 0.015 & 0.027 \\
\hline 04170000 & 08-05-2003 & 0.55 & 0.06 & 0.62 & 0.023 & $<.02$ & 0.011 & 0.025 \\
\hline 04170000 & 09-08-2003 & 0.42 & $<.41$ & 0.46 & E.005 & $<.18$ & 0.009 & 0.021 \\
\hline 04170500 & 09-18-2001 & 0.51 & $<.04$ & $<.05$ & $<.006$ & $<.02$ & 0.007 & 0.006 \\
\hline 04170500 & 11-19-2001 & 0.41 & E.04 & 0.26 & 0.01 & $<.02$ & 0.005 & 0.004 \\
\hline 04170500 & 01-23-2002 & 0.37 & 0.05 & 0.31 & E.005 & $<.02$ & E.004 & $<.004$ \\
\hline 04170500 & 03-13-2002 & 0.3 & $<.04$ & 0.27 & E.007 & $<.02$ & E.004 & E.002 \\
\hline 04170500 & 05-02-2002 & 0.5 & E.03 & $<.05$ & $<.008$ & $<.04$ & 0.006 & $<.06$ \\
\hline 04170500 & 06-05-2002 & 0.45 & E.03 & $<.05$ & $<.008$ & $<.02$ & 0.013 & $<.004$ \\
\hline 04170500 & 07-08-2002 & 0.49 & 0.04 & E.03 & $<.008$ & $<.02$ & 0.014 & $<.06$ \\
\hline 04170500 & 08-28-2002 & 0.53 & $<.04$ & $<.05$ & $<.008$ & $<.02$ & 0.007 & 0.027 \\
\hline 04170500 & $12-10-2002$ & 0.4 & E.04 & 0.19 & 0.024 & $<.02$ & E.004 & 0.015 \\
\hline 04170500 & 02-02-2003 & 0.4 & 0.06 & 0.28 & 0.016 & $<.02$ & E.004 & 0.011 \\
\hline 04170500 & 05-01-2003 & 0.6 & $<.04$ & 0.07 & E.007 & $<.02$ & E.004 & 0.017 \\
\hline 04170500 & $07-22-2003$ & 0.6 & E.04 & $<.06$ & $<.008$ & $<.02$ & 0.01 & 0.019 \\
\hline 04170500 & 08-05-2003 & 0.65 & E.02 & $<.06$ & $<.008$ & $<.02$ & 0.009 & 0.031 \\
\hline 04170500 & $09-08-2003$ & 0.73 & E.03 & $<.06$ & $<.008$ & $<.18$ & 0.01 & 0.022 \\
\hline
\end{tabular}


Table 9.Results of synoptic stream-water-quality sample analysis in and around Oakland County, Michigan between September 2001 and September 2003- continued

$[\mathrm{mg} / \mathrm{L}$, milligrams per liter; $\mathrm{mg} / \mathrm{L}$, micrograms per liter; $\mathrm{mS} / \mathrm{cm}$, microsiemens per centimeter; wf, filtered water; $\mathrm{mL}$, milliliter]

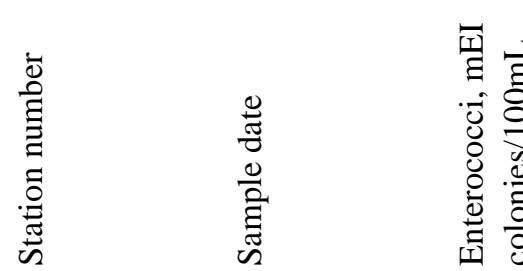

\begin{tabular}{llrrrr}
\hline 04170000 & $03-13-2002$ & -- & 5 & -- & -- \\
04170000 & $05-02-2002$ & -- & 16 & -- & -- \\
04170000 & $06-05-2002$ & -- & -- & -- & -- \\
04170000 & $07-08-2002$ & -- & -- & -- & -- \\
04170000 & $08-20-2002$ & -- & A480 & -- & -- \\
04170000 & $11-11-2002$ & -- & 210 & -- & -- \\
04170000 & $12-10-2002$ & -- & -- & -- & -- \\
04170000 & $02-02-2003$ & -- & 33 & -- & -- \\
04170000 & $04-22-2003$ & -- & -- & -- & -- \\
04170000 & $05-01-2003$ & -- & -- & -- & -- \\
04170000 & $06-12-2003$ & -- & 17 & -- & -- \\
04170000 & $07-22-2003$ & -- & -- & -- & -- \\
04170000 & $08-05-2003$ & 70 & -- & 20 & E190 \\
04170000 & $09-08-2003$ & 35 & -- & 23 & 33 \\
04170500 & $09-18-2001$ & -- & -- & -- & -- \\
04170500 & $11-19-2001$ & -- & -- & -- & -- \\
04170500 & $01-23-2002$ & -- & -- & -- & -- \\
04170500 & $03-13-2002$ & -- & 0 & -- & -- \\
04170500 & $05-02-2002$ & -- & 3 & -- & -- \\
04170500 & $06-05-2002$ & -- & 2 & -- & -- \\
04170500 & $07-08-2002$ & -- & -- & -- & -- \\
04170500 & $08-28-2002$ & -- & $>14$ & -- & -- \\
04170500 & $12-10-2002$ & -- & -- & -- & -- \\
04170500 & $02-02-2003$ & -- & -- & -- & -- \\
04170500 & $05-01-2003$ & -- & 0 & -- & -- \\
04170500 & $07-22-2003$ & -- & -- & -- & - \\
04170500 & $08-05-2003$ & 2 & -- & 3 & 6 \\
04170500 & $09-08-2003$ & 13 & -- & 3 & 3 \\
\hline
\end{tabular}


Table 10. Results of analysis for selected organic compounts at selected sites in Oakland County, Michigan

[All conentrations reported in micrograms per liter; --, not analysed; <, less than; E, estimated, but below minimum reporting level; M, compound confirmed to be present, but not quantifiable]

\begin{tabular}{|c|c|c|c|c|c|c|c|c|c|c|c|c|c|c|c|}
\hline & & & & 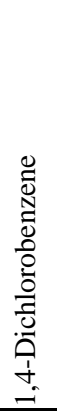 & 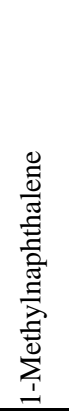 & 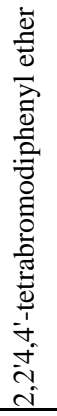 & 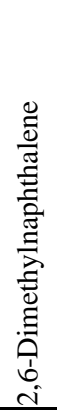 & 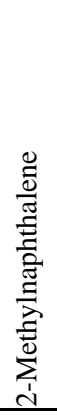 & 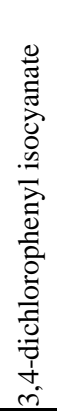 & 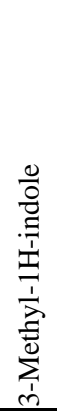 & 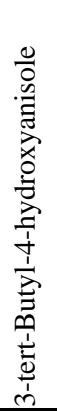 & 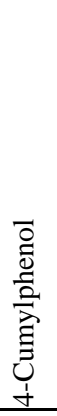 & 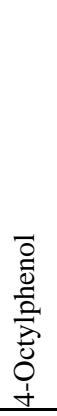 & 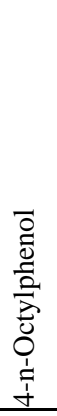 & 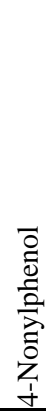 \\
\hline \multirow{12}{*}{ 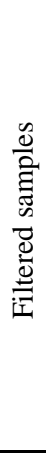 } & 04143830 & Aug. 21, 2002 & 1545 & $<.5$ & M & -- & $<.5$ & M & -- & $<1$ & $<5$ & $<1$ & $<1$ & -- & $<5$ \\
\hline & 04148035 & Aug. 21, 2002 & 1430 & $<.5$ & M & -- & $<.5$ & $\mathrm{M}$ & -- & $<1$ & $<5$ & $<1$ & $<1$ & -- & $<5$ \\
\hline & 04160800 & Aug. 20, 2002 & 850 & $<.5$ & M & -- & M & E.1 & -- & $<1$ & $<5$ & $<1$ & $<1$ & -- & E2 \\
\hline & 04160900 & Aug. 20, 2002 & 1130 & $<.5$ & M & -- & $<.5$ & $\mathrm{M}$ & -- & $<1$ & $<5$ & $<1$ & $<1$ & -- & $<5$ \\
\hline & 04161000 & Aug. 28, 2002 & 1345 & $<.5$ & $<.5$ & -- & $<.5$ & $<.5$ & -- & $<1$ & $<5$ & $<1$ & $<1$ & -- & $<5$ \\
\hline & 04161540 & Aug. 28, 2002 & 1230 & $<.5$ & $<.5$ & -- & $<.5$ & $<.5$ & -- & $<1$ & $<5$ & $<1$ & $<1$ & -- & $<5$ \\
\hline & 04161810 & Aug. 21, 2002 & 1100 & $<.5$ & $\mathrm{M}$ & -- & $<.5$ & M & -- & $<1$ & $<5$ & $<1$ & $<1$ & -- & $<5$ \\
\hline & 04166000 & Aug. 03, 2002 & 1030 & $<.5$ & $<.5$ & -- & $<.5$ & $<.5$ & -- & $<1$ & $<5$ & $<1$ & $<1$ & -- & E1 \\
\hline & 04166100 & Aug. 20, 2002 & 1400 & $<.5$ & $<.5$ & -- & $<.5$ & $<.5$ & -- & $<1$ & $<5$ & $<1$ & $<1$ & -- & $<5$ \\
\hline & 04166315 & Aug. 21, 2002 & 815 & $<.5$ & $\mathrm{M}$ & -- & $<.5$ & E. 1 & -- & $<1$ & $<5$ & $<1$ & $<1$ & -- & $<5$ \\
\hline & 04170000 & Aug. 20, 2002 & 1200 & M & $\mathrm{M}$ & -- & $<.5$ & E. 1 & -- & $<1$ & $<5$ & $<1$ & $<1$ & -- & E1 \\
\hline & 04170500 & Aug. 28, 2002 & 800 & $<.5$ & $<.5$ & -- & $<.5$ & $<.5$ & -- & $<1$ & $<5$ & $<1$ & $<1$ & -- & $<5$ \\
\hline \multirow{14}{*}{ 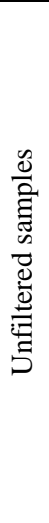 } & 04143830 & Sept. 09, 2003 & 900 & $\overline{--}$ & $<.5$ & $<.5$ & $<.5$ & $<.5$ & $<.5$ & $<1$ & $<5$ & $<1$ & $\overline{--}$ & $<1$ & $<5$ \\
\hline & 04148035 & Sept. 09, 2003 & 1000 & -- & $<.5$ & $<.5$ & $<.5$ & $<.5$ & M & $<1$ & $<5$ & $<1$ & -- & $<1$ & $<5$ \\
\hline & 04160800 & Sept. 09, 2003 & 1130 & -- & $<.5$ & $<.5$ & $<.5$ & $<.5$ & $<.5$ & $<1$ & $<5$ & $<1$ & -- & $<1$ & $<5$ \\
\hline & 04160900 & Sept. 08, 2003 & 1600 & -- & $<.5$ & $<.5$ & $<.5$ & $<.5$ & $<.5$ & $<1$ & $<5$ & $<1$ & -- & $<1$ & $<5$ \\
\hline & 04161000 & Sept. 08, 2003 & 1500 & -- & $<.5$ & $<.5$ & $<.5$ & $<.5$ & $<.5$ & $<1$ & $<5$ & $<1$ & -- & $<1$ & $<5$ \\
\hline & 04161540 & Sept. 09, 2003 & 1245 & -- & $<.5$ & $<.5$ & $<.5$ & $<.5$ & $\mathrm{M}$ & $<1$ & $<5$ & $<1$ & -- & $<1$ & $<5$ \\
\hline & 04161580 & Sept. 09, 2003 & 1545 & -- & $<.5$ & $<.5$ & $<.5$ & $<.5$ & $\mathrm{M}$ & $<1$ & $<5$ & $<1$ & -- & $<1$ & $<5$ \\
\hline & 04161810 & Sept. 09, 2003 & 1415 & -- & $<.5$ & $<.5$ & $<.5$ & $<.5$ & $<.5$ & $<1$ & $<5$ & $<1$ & -- & $<1$ & $<5$ \\
\hline & 04166000 & Sept. 08, 2003 & 1400 & -- & $<.5$ & $<.5$ & $<.5$ & $<.5$ & E. 2 & $<1$ & $<5$ & $<1$ & -- & $<1$ & $<5$ \\
\hline & 04166100 & Sept. 08, 2003 & 1045 & -- & $<.5$ & $<.5$ & $<.5$ & $<.5$ & E. 1 & $<1$ & $<5$ & $<1$ & -- & $<1$ & $<5$ \\
\hline & 04166200 & Sept. 08, 2003 & 1300 & -- & $<.5$ & $<.5$ & $<.5$ & $<.5$ & $<.5$ & $<1$ & $<5$ & $<1$ & -- & $<1$ & $<5$ \\
\hline & 04166315 & Sept. 08, 2003 & 1145 & -- & $<.5$ & $<.5$ & $<.5$ & $<.5$ & $<.5$ & $<1$ & $<5$ & $<1$ & -- & $<1$ & $<5$ \\
\hline & 04170000 & Sept. 08, 2003 & 945 & -- & $<.5$ & $<.5$ & $<.5$ & $<.5$ & $<.5$ & $<1$ & $<5$ & $<1$ & -- & $<1$ & $<5$ \\
\hline & 04170500 & Sept. 08, 2003 & 845 & -- & $\mathrm{M}$ & $<.5$ & $<.5$ & $\mathrm{M}$ & $<.5$ & $<1$ & $<5$ & $<1$ & -- & $<1$ & $<5$ \\
\hline
\end{tabular}


Table 10. Results of analysis for selected organic compounts at selected sites in Oakland County, Michigan - continued

[All conentrations reported in micrograms per liter; --, not analysed; <, less than; E, estimated, but below minimum reporting level; M, compound confirmed to be present, but not quantifiable]

\begin{tabular}{|c|c|c|c|c|c|c|c|c|c|c|c|c|c|c|c|}
\hline & & & 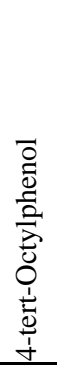 & 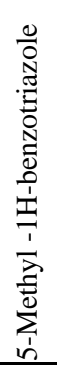 & 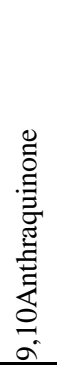 & 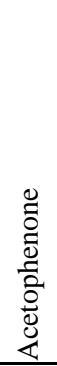 & 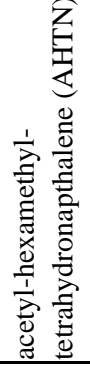 & 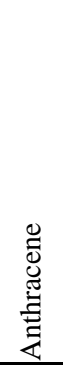 & 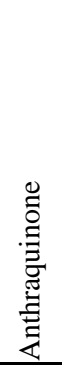 & 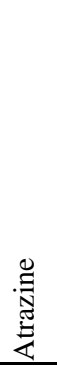 & 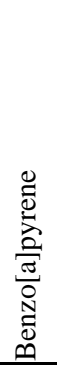 & 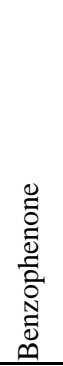 & 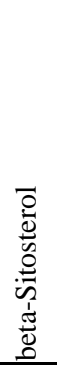 & 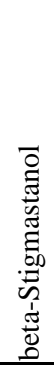 & 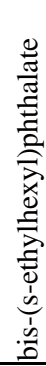 \\
\hline \multirow{12}{*}{ 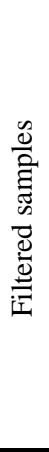 } & 04143830 & Aug. 21, 2002 & $<1$ & $<2$ & $<.5$ & $<.5$ & E.1 & $<.5$ & -- & -- & $<.5$ & E. 2 & $<2$ & $<2$ & -- \\
\hline & 04148035 & Aug. 21, 2002 & $<1$ & $<2$ & $<.5$ & $<.5$ & $<.5$ & M & -- & -- & M & E. 3 & $<2$ & $<2$ & -- \\
\hline & 04160800 & Aug. 20, 2002 & $<1$ & $<2$ & $<.5$ & $<.5$ & $<.5$ & $<.5$ & -- & -- & $<.5$ & E. 2 & E1 & $<2$ & -- \\
\hline & 04160900 & Aug. 20, 2002 & $<1$ & $<2$ & $<.5$ & $<.5$ & $<.5$ & M & -- & -- & M & E.1 & M & $<2$ & -- \\
\hline & 04161000 & Aug. 28, 2002 & $<1$ & $<2$ & $<.5$ & $<.5$ & E. 3 & $<.5$ & -- & -- & $<.5$ & E. 2 & $<2$ & $<2$ & -- \\
\hline & 04161540 & Aug. 28, 2002 & $<1$ & $<2$ & $<.5$ & $<.5$ & $<.5$ & $<.5$ & -- & -- & $<.5$ & E.1 & $<2$ & $<2$ & -- \\
\hline & 04161810 & Aug. 21, 2002 & $<1$ & $<2$ & $\mathrm{M}$ & $<.5$ & $\mathrm{M}$ & $\mathrm{M}$ & -- & -- & $<.5$ & E. 2 & $<2$ & $<2$ & -- \\
\hline & 04166000 & Aug. 03, 2002 & $<1$ & $<2$ & $<.5$ & $<.5$ & M & $<.5$ & -- & -- & $<.5$ & E. 2 & $<2$ & $<2$ & -- \\
\hline & 04166100 & Aug. 20, 2002 & $<1$ & $<2$ & E.1 & $<.5$ & $<.5$ & $<.5$ & -- & -- & $<.5$ & E.1 & $<2$ & $<2$ & -- \\
\hline & 04166315 & Aug. 21, 2002 & $<1$ & $<2$ & $\mathrm{M}$ & $<.5$ & $<.5$ & $<.5$ & -- & -- & $<.5$ & E. 2 & $<2$ & $<2$ & -- \\
\hline & 04170000 & Aug. 20, 2002 & $<1$ & $<2$ & $<.5$ & $<.5$ & E.1 & $<.5$ & -- & -- & $<.5$ & E. 3 & E1 & $<2$ & -- \\
\hline & 04170500 & Aug. 28, 2002 & $<1$ & $<2$ & $<.5$ & $<.5$ & $<.5$ & $<.5$ & -- & -- & $<.5$ & E. 2 & $<2$ & $<2$ & -- \\
\hline \multirow{14}{*}{ 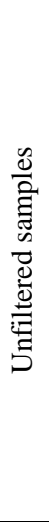 } & 04143830 & Sept. 09, 2003 & $<1$ & $<2$ & -- & $<.5$ & E.1 & $<.5$ & $<.5$ & $<.5$ & $<.5$ & $\mathrm{M}$ & $<2$ & $<2$ & $<2$ \\
\hline & 04148035 & Sept. 09, 2003 & $<1$ & $<2$ & -- & $<.5$ & $<.5$ & $<.5$ & $<.5$ & $<.5$ & $<.5$ & $<.5$ & $<2$ & $<2$ & $<2$ \\
\hline & 04160800 & Sept. 09, 2003 & M & $<2$ & -- & $<.5$ & $<.5$ & $<.5$ & $<.5$ & $<.5$ & $<.5$ & $<.5$ & $<2$ & $<2$ & E2 \\
\hline & 04160900 & Sept. 08, 2003 & M & $<2$ & -- & $<.5$ & $<.5$ & $<.5$ & $<.5$ & $<.5$ & $<.5$ & $<.5$ & $<2$ & $<2$ & $<2$ \\
\hline & 04161000 & Sept. 08, 2003 & M & $<2$ & -- & E. 1 & 0.6 & $<.5$ & E.1 & $<.5$ & $<.5$ & E.1 & $<2$ & $<2$ & $<2$ \\
\hline & 04161540 & Sept. 09, 2003 & M & $<2$ & -- & $<.5$ & $<.5$ & $<.5$ & $<.5$ & $<.5$ & $<.5$ & $<.5$ & $<2$ & $<2$ & $<2$ \\
\hline & 04161580 & Sept. 09, 2003 & $<1$ & $<2$ & -- & $<.5$ & $<.5$ & $<.5$ & $<.5$ & $<.5$ & $<.5$ & $<.5$ & $<2$ & $<2$ & $<2$ \\
\hline & 04161810 & Sept. 09, 2003 & M & $<2$ & -- & $<.5$ & E. 1 & $<.5$ & $<.5$ & $<.5$ & $<.5$ & M & $<2$ & $<2$ & $<2$ \\
\hline & 04166000 & Sept. 08, 2003 & $<1$ & $<2$ & -- & $<.5$ & $<.5$ & $<.5$ & E.1 & $<.5$ & $<.5$ & $\mathrm{M}$ & $<2$ & $<2$ & $<2$ \\
\hline & 04166100 & Sept. 08, 2003 & $<1$ & $<2$ & -- & $<.5$ & $<.5$ & $\mathrm{M}$ & E.1 & $<.5$ & $<.5$ & $<.5$ & $<2$ & $<2$ & $<2$ \\
\hline & 04166200 & Sept. 08, 2003 & M & $<2$ & -- & $<.5$ & $<.5$ & $\mathrm{M}$ & E. 1 & $<.5$ & $<.5$ & $\mathrm{M}$ & $<2$ & $<2$ & $<2$ \\
\hline & 04166315 & Sept. 08, 2003 & $<1$ & $<2$ & -- & $<.5$ & $<.5$ & $<.5$ & $<.5$ & $<.5$ & $<.5$ & $<.5$ & $<2$ & $<2$ & $<2$ \\
\hline & 04170000 & Sept. 08, 2003 & $\mathrm{M}$ & $<2$ & -- & $<.5$ & E.1 & $<.5$ & E.1 & $<.5$ & $<.5$ & $\mathrm{M}$ & $<2$ & $<2$ & $<2$ \\
\hline & 04170500 & Sept. 08, 2003 & $<1$ & $<2$ & -- & $<.5$ & $<.5$ & $<.5$ & $<.5$ & $\mathrm{M}$ & $<.5$ & $<.5$ & $<2$ & $<2$ & $<2$ \\
\hline
\end{tabular}


Table 10. Results of analysis for selected organic compounts at selected sites in Oakland County, Michigan - continued

[All conentrations reported in micrograms per liter; --, not analysed; <, less than; E, estimated, but below minimum reporting level; M, compound confirmed to be present, but not quantifiable]

\begin{tabular}{|c|c|c|c|c|c|c|c|c|c|c|c|c|c|c|c|}
\hline & & & 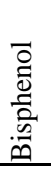 & 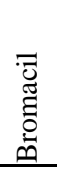 & : & 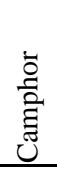 & 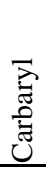 & 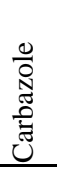 & $\begin{array}{l}0 \\
0 \\
0 \\
0 \\
0 \\
0 \\
0 \\
0 \\
0\end{array}$ & $\begin{array}{l}\overrightarrow{0} \\
\frac{0}{0} \\
\frac{0}{0} \\
0 \\
0 \\
0\end{array}$ & 苛 & 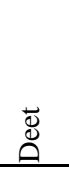 & 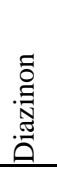 & 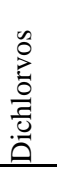 & 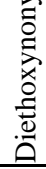 \\
\hline \multirow{12}{*}{ 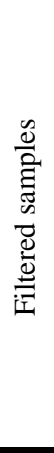 } & 04143830 & Aug. 21, 2002 & $<1$ & $<.5$ & $<.5$ & $<.5$ & $<1$ & $<.5$ & $<.5$ & $<2$ & $<1.00$ & E.1 & $<.5$ & -- & $<5$ \\
\hline & 04148035 & Aug. 21, 2002 & $<1$ & $<.5$ & $<.5$ & $<.5$ & $<1$ & $<.5$ & $<.5$ & $<2$ & $<1.00$ & E.1 & $<.5$ & -- & $<5$ \\
\hline & 04160800 & Aug. 20, 2002 & $<1$ & $<.5$ & $<.5$ & $<.5$ & $<1$ & $<.5$ & $<.5$ & E1 & $<1.00$ & E. 2 & $<.5$ & -- & M \\
\hline & 04160900 & Aug. 20, 2002 & $<1$ & $<.5$ & M & $<.5$ & $<1$ & $<.5$ & $<.5$ & M & $<1.00$ & E.1 & $<.5$ & -- & $<5$ \\
\hline & 04161000 & Aug. 28, 2002 & M & $<.5$ & M & $<.5$ & $<1$ & $<.5$ & $<.5$ & $<2$ & $<1.00$ & E.1 & $<.5$ & -- & $<5$ \\
\hline & 04161540 & Aug. 28, 2002 & $<1$ & $<.5$ & $<.5$ & $<.5$ & $<1$ & $<.5$ & $<.5$ & E1 & $<1.00$ & M & $<.5$ & -- & $<5$ \\
\hline & 04161810 & Aug. 21, 2002 & $<1$ & $<.5$ & E.1 & $<.5$ & $<1$ & $\mathrm{M}$ & $<.5$ & $<2$ & E.0700 & E. 2 & $<.5$ & -- & $<5$ \\
\hline & 04166000 & Aug. 03, 2002 & M & $<.5$ & $<.5$ & M & $<1$ & $<.5$ & $<.5$ & M & $<1.00$ & E. 2 & $<.5$ & -- & M \\
\hline & 04166100 & Aug. 20, 2002 & $<1$ & $<.5$ & E. 3 & $<.5$ & $<1$ & $<.5$ & $<.5$ & M & $<1.00$ & E. 2 & $<.5$ & -- & $<5$ \\
\hline & 04166315 & Aug. 21, 2002 & $<1$ & $<.5$ & E.1 & $<.5$ & $<1$ & $<.5$ & $<.5$ & $<2$ & $<1.00$ & E. 2 & M & -- & $<5$ \\
\hline & 04170000 & Aug. 20, 2002 & $<1$ & $<.5$ & M & $<.5$ & $<1$ & $<.5$ & $<.5$ & E2 & $<1.00$ & E. 2 & $<.5$ & -- & M \\
\hline & 04170500 & Aug. 28, 2002 & $<1$ & $<.5$ & $<.5$ & $<.5$ & $<1$ & $<.5$ & $<.5$ & $<2$ & $<1.00$ & E. 2 & $<.5$ & -- & $<5$ \\
\hline \multirow{14}{*}{ 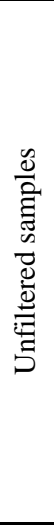 } & 04143830 & Sept. 09, 2003 & $<1$ & $<.5$ & $\mathrm{M}$ & $<.5$ & $<1$ & $<.5$ & $<.5$ & $<2$ & $<1$ & E.1 & $<.5$ & $<1$ & $<5$ \\
\hline & 04148035 & Sept. 09, 2003 & $<1$ & $<.5$ & $<.5$ & $<.5$ & $<1$ & $<.5$ & $<.5$ & $<2$ & $<1$ & M & $<.5$ & $<1$ & $<5$ \\
\hline & 04160800 & Sept. 09, 2003 & $<1$ & $<.5$ & $<.5$ & $<.5$ & $<1$ & $<.5$ & $<.5$ & $<2$ & $<1$ & E.1 & $<.5$ & $<1$ & $<5$ \\
\hline & 04160900 & Sept. 08, 2003 & $<1$ & $<.5$ & $<.5$ & $<.5$ & $<1$ & $<.5$ & $<.5$ & $<2$ & $<1$ & E.1 & $<.5$ & $<1$ & $<5$ \\
\hline & 04161000 & Sept. 08, 2003 & M & $<.5$ & M & $<.5$ & $<1$ & $<.5$ & $<.5$ & $<2$ & $<1$ & E.1 & $<.5$ & $<1$ & $<5$ \\
\hline & 04161540 & Sept. 09, 2003 & $<1$ & $<.5$ & $<.5$ & $<.5$ & $<1$ & $<.5$ & $<.5$ & $<2$ & $<1$ & $\mathrm{M}$ & $<.5$ & $<1$ & $<5$ \\
\hline & 04161580 & Sept. 09, 2003 & $<1$ & $<.5$ & $<.5$ & $<.5$ & $<1$ & $<.5$ & $<.5$ & $<2$ & $<1$ & M & $<.5$ & $<1$ & $<5$ \\
\hline & 04161810 & Sept. 09, 2003 & $<1$ & $<.5$ & M & $<.5$ & $<1$ & $<.5$ & $<.5$ & $<2$ & $<1$ & E.1 & $<.5$ & $<1$ & $<5$ \\
\hline & 04166000 & Sept. 08, 2003 & $<1$ & $<.5$ & E.1 & $<.5$ & $<1$ & $<.5$ & $<.5$ & E1 & $<1$ & E.1 & $<.5$ & $<1$ & $<5$ \\
\hline & 04166100 & Sept. 08, 2003 & $<1$ & $<.5$ & M & $<.5$ & $<1$ & $<.5$ & $<.5$ & E1 & $<1$ & E.1 & $<.5$ & $<1$ & $<5$ \\
\hline & 04166200 & Sept. 08, 2003 & $<1$ & $<.5$ & E.1 & $<.5$ & $<1$ & $<.5$ & $<.5$ & E1 & $<1$ & E. 1 & $<.5$ & $<1$ & $<5$ \\
\hline & 04166315 & Sept. 08, 2003 & $<1$ & $<.5$ & $<.5$ & $<.5$ & $<1$ & $<.5$ & $<.5$ & $<2$ & $<1$ & M & $<.5$ & $<1$ & $<5$ \\
\hline & 04170000 & Sept. 08, 2003 & $<1$ & $<.5$ & E.1 & E.1 & $<1$ & $<.5$ & $<.5$ & $<2$ & $<1$ & E.1 & $<.5$ & $<1$ & $<5$ \\
\hline & 04170500 & Sept. 08, 2003 & $<1$ & $<.5$ & $<.5$ & $<.5$ & $<1$ & $<.5$ & $<.5$ & $<2$ & $<1$ & E.1 & $<.5$ & $<1$ & $<5$ \\
\hline
\end{tabular}


Table 10. Results of analysis for selected organic compounts at selected sites in Oakland County, Michigan - continued

[All conentrations reported in micrograms per liter; --, not analysed; <, less than; E, estimated, but below minimum reporting level; M, compound confirmed to be present, but not quantifiable]

\begin{tabular}{|c|c|c|c|c|c|c|c|c|c|c|c|c|c|c|c|}
\hline & & & 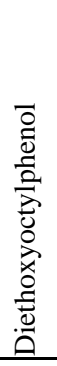 & 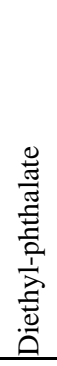 & 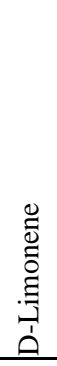 & 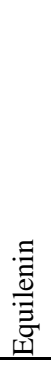 & 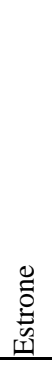 & 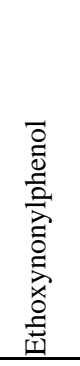 & 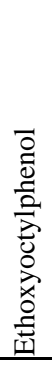 & 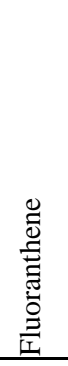 & 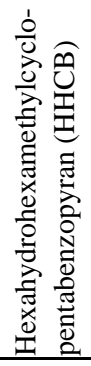 & $\begin{array}{l}\frac{0}{0} \\
\stackrel{0}{\Xi}\end{array}$ & 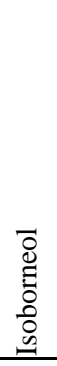 & $\begin{array}{l}0 \\
\tilde{0} \\
0 \\
0 \\
\stackrel{0}{0} \\
0 \\
0 \\
0\end{array}$ & 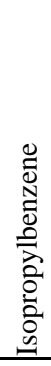 \\
\hline \multirow{12}{*}{ 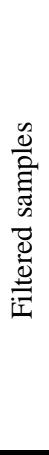 } & 04143830 & Aug. 21, 2002 & $<1$ & -- & $<.5$ & $<5$ & $<5$ & -- & $<1$ & M & M & $<.5$ & $<.5$ & $<.5$ & $<.5$ \\
\hline & 04148035 & Aug. 21, 2002 & $<1$ & -- & $<.5$ & $<5$ & $<5$ & -- & $<1$ & M & $<.5$ & $<.5$ & $<.5$ & $<.5$ & $<.5$ \\
\hline & 04160800 & Aug. 20, 2002 & $<1$ & -- & E. 1 & $<5$ & $<5$ & -- & $<1$ & $<.5$ & $<.5$ & $<.5$ & $<.5$ & 3.1 & $<.5$ \\
\hline & 04160900 & Aug. 20, 2002 & $<1$ & -- & $<.5$ & $<5$ & $<5$ & -- & $<1$ & M & $<.5$ & $<.5$ & $<.5$ & $<.5$ & $<.5$ \\
\hline & 04161000 & Aug. 28, 2002 & $<1$ & -- & $<.5$ & $<5$ & $<5$ & -- & $<1$ & $<.5$ & M & $<.5$ & $<.5$ & $<.5$ & $<.5$ \\
\hline & 04161540 & Aug. 28, 2002 & $<1$ & -- & $<.5$ & $<5$ & $<5$ & -- & $<1$ & $<.5$ & $<.5$ & $<.5$ & $<.5$ & $<.5$ & $<.5$ \\
\hline & 04161810 & Aug. 21, 2002 & $<1$ & -- & $<.5$ & $<5$ & $<5$ & -- & $<1$ & M & M & $<.5$ & $<.5$ & $<.5$ & $<.5$ \\
\hline & 04166000 & Aug. 03, 2002 & $<1$ & -- & $<.5$ & $<5$ & $<5$ & -- & M & $<.5$ & $<.5$ & E.1 & $<.5$ & E. 3 & $<.5$ \\
\hline & 04166100 & Aug. 20, 2002 & $<1$ & -- & $<.5$ & $<5$ & $<5$ & -- & $<1$ & $<.5$ & $<.5$ & $<.5$ & $<.5$ & $<.5$ & $<.5$ \\
\hline & 04166315 & Aug. 21, 2002 & $<1$ & -- & $<.5$ & $<5$ & $<5$ & -- & $<1$ & M & $<.5$ & $<.5$ & $<.5$ & $<.5$ & $<.5$ \\
\hline & 04170000 & Aug. 20, 2002 & $<1$ & -- & E.1 & $<5$ & $<5$ & -- & $<1$ & M & $\mathrm{M}$ & $<.5$ & $<.5$ & $<.5$ & $\mathrm{M}$ \\
\hline & 04170500 & Aug. 28, 2002 & $<1$ & -- & $<.5$ & $<5$ & $<5$ & -- & $<1$ & $<.5$ & $<.5$ & $<.5$ & $<.5$ & $<.5$ & $<.5$ \\
\hline \multirow{14}{*}{ 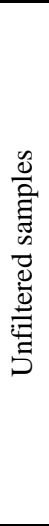 } & 04143830 & Sept. 09, 2003 & $<1$ & $<.5$ & $<.5$ & $<5$ & $<5$ & $<2.00$ & $<1$ & $<.5$ & $\mathrm{M}$ & $<.5$ & $<.5$ & $<.5$ & -- \\
\hline & 04148035 & Sept. 09, 2003 & $<1$ & $<.5$ & $<.5$ & $<5$ & $<5$ & $<2.00$ & $<1$ & $<.5$ & $<.5$ & $<.5$ & $<.5$ & $<.5$ & -- \\
\hline & 04160800 & Sept. 09, 2003 & $<1$ & $<.5$ & $<.5$ & $<5$ & $<5$ & $<2.00$ & $<1$ & $<.5$ & $<.5$ & $<.5$ & $<.5$ & $<.5$ & -- \\
\hline & 04160900 & Sept. 08, 2003 & $<1$ & $<.5$ & $<.5$ & $<5$ & $<5$ & $<2.00$ & $<1$ & $<.5$ & $<.5$ & $<.5$ & $<.5$ & $<.5$ & -- \\
\hline & 04161000 & Sept. 08, 2003 & $<1$ & $<.5$ & $<.5$ & $<5$ & $<5$ & $<2.00$ & $<1$ & $\mathrm{M}$ & E.1 & $<.5$ & $<.5$ & $<.5$ & -- \\
\hline & 04161540 & Sept. 09, 2003 & $<1$ & $<.5$ & $<.5$ & $<5$ & $<5$ & $<2.00$ & $<1$ & $<.5$ & $<.5$ & $<.5$ & $<.5$ & $<.5$ & -- \\
\hline & 04161580 & Sept. 09, 2003 & $<1$ & $<.5$ & $<.5$ & $<5$ & $<5$ & $<2.00$ & $<1$ & $<.5$ & $<.5$ & $<.5$ & $<.5$ & $<.5$ & -- \\
\hline & 04161810 & Sept. 09, 2003 & $<1$ & $<.5$ & $<.5$ & $<5$ & $<5$ & $<2.00$ & $<1$ & $\mathrm{M}$ & M & $<.5$ & $<.5$ & $<.5$ & -- \\
\hline & 04166000 & Sept. 08, 2003 & $<1$ & $<.5$ & $<.5$ & $<5$ & $<5$ & $<2.00$ & $<1$ & M & $<.5$ & $<.5$ & $<.5$ & $<.5$ & -- \\
\hline & 04166100 & Sept. 08, 2003 & $<1$ & $<.5$ & $<.5$ & $<5$ & $<5$ & $<2.00$ & $<1$ & E.1 & $<.5$ & $<.5$ & $<.5$ & $<.5$ & -- \\
\hline & 04166200 & Sept. 08, 2003 & $<1$ & $<.5$ & $<.5$ & $<5$ & $<5$ & $<2.00$ & $<1$ & E.1 & $<.5$ & $<.5$ & $<.5$ & $<.5$ & -- \\
\hline & 04166315 & Sept. 08, 2003 & $<1$ & $<.5$ & $<.5$ & $<5$ & $<5$ & $<2.00$ & $<1$ & M & $<.5$ & $<.5$ & $<.5$ & $<.5$ & -- \\
\hline & 04170000 & Sept. 08, 2003 & $<1$ & $<.5$ & $<.5$ & $<5$ & $<5$ & $<2.00$ & $<1$ & $<.5$ & M & $<.5$ & $<.5$ & $<.5$ & -- \\
\hline & 04170500 & Sept. 08, 2003 & $<1$ & $<.5$ & $<.5$ & $<5$ & $<5$ & $<2.00$ & $<1$ & $<.5$ & $<.5$ & $<.5$ & $<.5$ & $<.5$ & -- \\
\hline
\end{tabular}


Table 10. Results of analysis for selected organic compounts at selected sites in Oakland County, Michigan - continued

[All conentrations reported in micrograms per liter; --, not analysed; <, less than; E, estimated, but below minimum reporting level; M, compound confirmed to be present, but not quantifiable]

\begin{tabular}{|c|c|c|c|c|c|c|c|c|c|c|c|c|c|c|c|}
\hline & & & 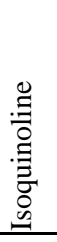 & $\begin{array}{l}\overrightarrow{0} \\
\overline{0} \\
\overline{0} \\
\Sigma\end{array}$ & 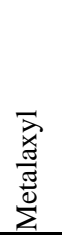 & 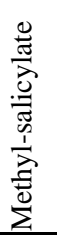 & $\begin{array}{l}\frac{\dot{o}}{0} \\
\frac{0}{0} \\
\frac{\tilde{\sigma}}{0} \\
\stackrel{0}{0}\end{array}$ & 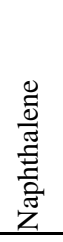 & 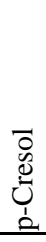 & 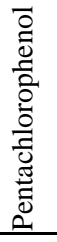 & 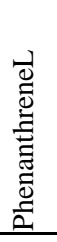 & 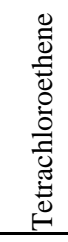 & 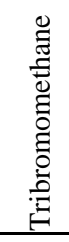 & 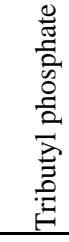 & 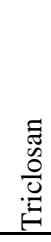 \\
\hline \multirow{12}{*}{ 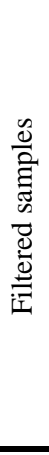 } & 04143830 & Aug. 21, 2002 & $<.5$ & $<.5$ & $<.5$ & $<.5$ & $<.5$ & E.1 & $<1$ & $<2$ & $\mathrm{M}$ & $<.5$ & $<.5$ & M & $<1$ \\
\hline & 04148035 & Aug. 21, 2002 & $<.5$ & $<.5$ & $<.5$ & $<.5$ & $<.5$ & E.1 & $<1$ & $<2$ & M & $<.5$ & $<.5$ & M & $<1$ \\
\hline & 04160800 & Aug. 20, 2002 & $<.5$ & $<.4$ & $<.5$ & $<.5$ & $<.5$ & E.1 & $<1$ & $<2$ & M & $<.5$ & $<.5$ & $<.5$ & $<1$ \\
\hline & 04160900 & Aug. 20, 2002 & $<.5$ & $<.5$ & $<.5$ & $<.5$ & $<.5$ & E. 1 & $<1$ & $<2$ & M & $<.5$ & $<.5$ & $<.5$ & $<1$ \\
\hline & 04161000 & Aug. 28, 2002 & $<.5$ & $<.5$ & $<.5$ & $<.5$ & $<.5$ & $<.5$ & $<1$ & $<2$ & $<.5$ & $<.5$ & $<.5$ & E.1 & $<1$ \\
\hline & 04161540 & Aug. 28, 2002 & $<.5$ & $<.5$ & $<.5$ & $<.5$ & $<.5$ & $<.5$ & $<1$ & $<2$ & $<.5$ & $<.5$ & M & $<.5$ & $<1$ \\
\hline & 04161810 & Aug. 21, 2002 & $<.5$ & $<.5$ & $<.5$ & $<.5$ & M & E. 1 & $<1$ & $<2$ & M & $<.5$ & $<.5$ & M & $<1$ \\
\hline & 04166000 & Aug. 03, 2002 & $<.5$ & $<.5$ & $<.5$ & $<.5$ & $<.5$ & $<.5$ & M & $<2$ & $<.5$ & $<.5$ & $<.5$ & $<.5$ & M \\
\hline & 04166100 & Aug. 20, 2002 & $<.5$ & $<.5$ & $<.5$ & $<.5$ & $<.5$ & $<.5$ & $<1$ & $<2$ & $<.5$ & $<.5$ & $<.5$ & M & $<1$ \\
\hline & 04166315 & Aug. 21, 2002 & $<.5$ & $<.5$ & $<.5$ & $<.5$ & $<.5$ & E.1 & $<1$ & $<2$ & $\mathrm{M}$ & $<.5$ & $<.5$ & M & $<1$ \\
\hline & 04170000 & Aug. 20, 2002 & $<.5$ & $<.4$ & $<.5$ & $<.5$ & $<.5$ & E.1 & $<1$ & $<2$ & M & $<.5$ & $<.5$ & $<.5$ & $<1$ \\
\hline & 04170500 & Aug. 28, 2002 & $<.5$ & $<.5$ & $<.5$ & $<.5$ & $<.5$ & $<.5$ & $<1$ & $<2$ & $<.5$ & $<.5$ & $<.5$ & $<.5$ & $<1$ \\
\hline \multirow{14}{*}{ 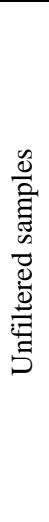 } & 04143830 & Sept. 09, 2003 & $<.5$ & $<.5$ & $<.5$ & $<.5$ & $<.5$ & -- & M & $<2$ & $\mathrm{M}$ & $<.5$ & $<.5$ & $\mathrm{M}$ & $<1$ \\
\hline & 04148035 & Sept. 09, 2003 & $<.5$ & $<.5$ & $<.5$ & $<.5$ & $<.5$ & -- & $<1$ & $<2$ & M & $<.5$ & $<.5$ & $<.5$ & $<1$ \\
\hline & 04160800 & Sept. 09, 2003 & $<.5$ & $<.5$ & $<.5$ & $<.5$ & $<.5$ & -- & M & $<2$ & $<.5$ & $<.5$ & $<.5$ & $<.5$ & $<1$ \\
\hline & 04160900 & Sept. 08, 2003 & $<.5$ & $<.5$ & $<.5$ & $<.5$ & $<.5$ & -- & $<1$ & $<2$ & M & $<.5$ & $<.5$ & $<.5$ & $<1$ \\
\hline & 04161000 & Sept. 08, 2003 & $<.5$ & $<.5$ & $<.5$ & $<.5$ & $<.5$ & -- & $<1$ & $<2$ & M & $<.5$ & $\mathrm{M}$ & E. 1 & $<1$ \\
\hline & 04161540 & Sept. 09, 2003 & $<.5$ & $<.5$ & $<.5$ & $<.5$ & $<.5$ & -- & $<1$ & $<2$ & M & $<.5$ & $<.5$ & $<.5$ & $<1$ \\
\hline & 04161580 & Sept. 09, 2003 & $<.5$ & $<.5$ & $<.5$ & $<.5$ & $<.5$ & -- & $<1$ & $<2$ & $<.5$ & $<.5$ & $<.5$ & $<.5$ & $<1$ \\
\hline & 04161810 & Sept. 09, 2003 & $<.5$ & $<.5$ & $<.5$ & $<.5$ & $<.5$ & -- & $<1$ & $<2$ & M & $<.5$ & $<.5$ & M & $<1$ \\
\hline & 04166000 & Sept. 08, 2003 & $<.5$ & $<.5$ & $<.5$ & $<.5$ & $<.5$ & -- & $<1$ & $<2$ & M & $<.5$ & $<.5$ & $<.5$ & $<1$ \\
\hline & 04166100 & Sept. 08, 2003 & $<.5$ & $<.5$ & $<.5$ & $<.5$ & $<.5$ & -- & $<1$ & $<2$ & M & $<.5$ & $<.5$ & $<.5$ & $<1$ \\
\hline & 04166200 & Sept. 08, 2003 & $<.5$ & $<.5$ & $<.5$ & $<.5$ & $<.5$ & -- & $<1$ & $<2$ & M & E.1 & $<.5$ & M & $<1$ \\
\hline & 04166315 & Sept. 08, 2003 & $<.5$ & $<.5$ & $<.5$ & $<.5$ & $<.5$ & -- & $<1$ & $<2$ & $<.5$ & $<.5$ & $<.5$ & $<.5$ & $<1$ \\
\hline & 04170000 & Sept. 08, 2003 & $<.5$ & $<.5$ & $<.5$ & $<.5$ & $<.5$ & -- & M & $<2$ & $<.5$ & $<.5$ & $<.5$ & $<.5$ & $<1$ \\
\hline & 04170500 & Sept. 08, 2003 & $<.5$ & $<.5$ & $<.5$ & $<.5$ & $<.5$ & -- & $<1$ & $<2$ & $<.5$ & $<.5$ & $<.5$ & $<.5$ & $<1$ \\
\hline
\end{tabular}


Table 10. Results of analysis for selected organic compounts at selected sites in Oakland County, Michigan - continued

[All conentrations reported in micrograms per liter; --, not analysed; <, less than; E, estimated, but below minimum reporting level; M, compound confirmed to be present, but not quantifiable]

\begin{tabular}{|c|c|c|c|c|c|c|c|c|c|c|c|c|}
\hline & & & 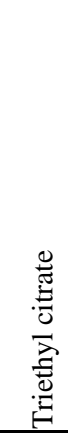 & 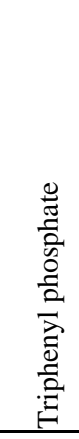 & 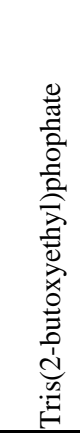 & 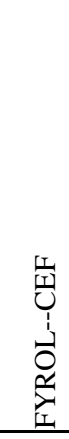 & 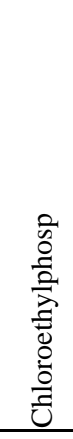 & 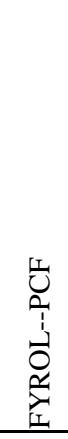 & 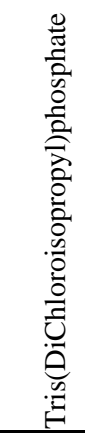 & 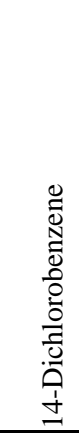 & 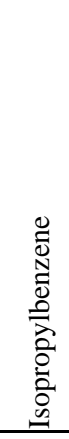 & 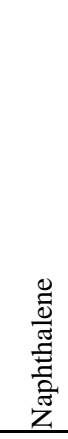 \\
\hline \multirow{12}{*}{ 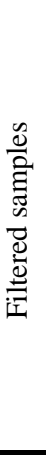 } & 04143830 & Aug. 21, 2002 & $<.5$ & M & E. 2 & M & -- & $\mathrm{M}$ & -- & -- & -- & -- \\
\hline & 04148035 & Aug. 21, 2002 & $<.5$ & M & $<.5$ & $<.5$ & -- & $<.5$ & -- & -- & -- & -- \\
\hline & 04160800 & Aug. 20, 2002 & $<.5$ & M & E. 4 & E. 1 & -- & $<.5$ & -- & -- & -- & -- \\
\hline & 04160900 & Aug. 20, 2002 & $<.5$ & $\mathrm{M}$ & $<.5$ & $\mathrm{M}$ & -- & $<.5$ & -- & -- & -- & -- \\
\hline & 04161000 & Aug. 28, 2002 & E.1 & $<.5$ & E.1 & E.1 & -- & E.1 & -- & -- & -- & -- \\
\hline & 04161540 & Aug. 28, 2002 & $<.5$ & $<.5$ & $\mathrm{M}$ & $<.5$ & -- & $<.5$ & -- & -- & -- & -- \\
\hline & 04161810 & Aug. 21, 2002 & $\mathrm{M}$ & $\mathrm{M}$ & E.1 & E.1 & -- & E.1 & -- & -- & -- & -- \\
\hline & 04166000 & Aug. 03, 2002 & $<.5$ & $<.5$ & E.5 & M & -- & $\mathrm{M}$ & -- & -- & -- & -- \\
\hline & 04166100 & Aug. 20, 2002 & $<.5$ & $<.5$ & 1.3 & $\mathrm{M}$ & -- & $<.5$ & -- & -- & -- & -- \\
\hline & 04166315 & Aug. 21, 2002 & $<.5$ & $\mathrm{M}$ & 0.6 & E.1 & -- & $\mathrm{M}$ & -- & -- & -- & -- \\
\hline & 04170000 & Aug. 20, 2002 & $<.5$ & $\mathrm{M}$ & E.1 & E.1 & -- & E.1 & -- & -- & -- & -- \\
\hline & 04170500 & Aug. 28, 2002 & $<.5$ & $<.5$ & E.1 & $\mathrm{M}$ & -- & $\mathrm{M}$ & -- & -- & -- & -- \\
\hline \multirow{14}{*}{ 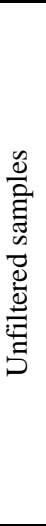 } & 04143830 & Sept. 09, 2003 & $\mathrm{M}$ & $<.5$ & E.2 & $\overline{--}$ & $\mathrm{M}$ & $\overline{--}$ & $\mathrm{M}$ & $<.5$ & $<.5$ & $<.5$ \\
\hline & 04148035 & Sept. 09, 2003 & $<.5$ & $<.5$ & $<.5$ & -- & $<.5$ & -- & $<.5$ & $\mathrm{M}$ & $<.5$ & $<.5$ \\
\hline & 04160800 & Sept. 09, 2003 & $<.5$ & $<.5$ & $<.5$ & -- & $<.5$ & -- & $<.5$ & $\mathrm{M}$ & $<.5$ & $<.5$ \\
\hline & 04160900 & Sept. 08, 2003 & $<.5$ & $<.5$ & $<.5$ & -- & $<.5$ & -- & $<.5$ & $<.5$ & $<.5$ & $<.5$ \\
\hline & 04161000 & Sept. 08, 2003 & E.1 & $\mathrm{M}$ & E. 3 & -- & E.1 & -- & E.1 & E.1 & $<.5$ & $<.5$ \\
\hline & 04161540 & Sept. 09, 2003 & $<.5$ & $<.5$ & $<.5$ & -- & $<.5$ & -- & $<.5$ & M & $<.5$ & $<.5$ \\
\hline & 04161580 & Sept. 09, 2003 & $<.5$ & $<.5$ & E. 2 & -- & $<.5$ & -- & $<.5$ & $<.5$ & $<.5$ & $<.5$ \\
\hline & 04161810 & Sept. 09, 2003 & $\mathrm{M}$ & $\mathrm{M}$ & E. 2 & -- & E.1 & -- & E.1 & $\mathrm{M}$ & $<.5$ & $<.5$ \\
\hline & 04166000 & Sept. 08, 2003 & $<.5$ & $<.5$ & E. 2 & -- & $\mathrm{M}$ & -- & $<.5$ & M & $<.5$ & $<.5$ \\
\hline & 04166100 & Sept. 08, 2003 & $<.5$ & $<.5$ & E. 4 & -- & $<.5$ & -- & $\mathrm{M}$ & M & $<.5$ & $<.5$ \\
\hline & 04166200 & Sept. 08, 2003 & $<.5$ & $\mathrm{M}$ & 0.5 & -- & $\mathrm{M}$ & -- & M & $\mathrm{M}$ & $<.5$ & $<.5$ \\
\hline & 04166315 & Sept. 08, 2003 & $<.5$ & $<.5$ & $<.5$ & -- & $\mathrm{M}$ & -- & M & $<.5$ & $<.5$ & $<.5$ \\
\hline & 04170000 & Sept. 08, 2003 & $<.5$ & $<.5$ & E.5 & -- & E.1 & -- & $\mathrm{M}$ & $<.5$ & $<.5$ & $<.5$ \\
\hline & 04170500 & Sept. 08, 2003 & $<.5$ & $<.5$ & $<.5$ & -- & $\mathrm{M}$ & -- & $<.5$ & $<.5$ & $<.5$ & $\mathrm{M}$ \\
\hline
\end{tabular}


Table 11. Vertical profiles of field-measured physical characteristics of selected lakes in Oakland County, Michigan measured during 2002 and 2003 $\left[\mathrm{mg} / \mathrm{L}\right.$, milligrams per liter; $\mathrm{ms} / \mathrm{cm}$, microsiemens per centimeter $;{ }^{\circ} \mathrm{C}$, degrees Celsius]

\begin{tabular}{|c|c|c|c|c|c|c|c|}
\hline Station Number & Sample Date & $\begin{array}{c}\text { Sample } \\
\text { Time }\end{array}$ & $\begin{array}{l}\text { Sampling } \\
\text { depth, in } \\
\text { feet }\end{array}$ & $\begin{array}{l}\text { Dissolved } \\
\text { oxygen in } \\
\mathrm{mg} / \mathrm{L}\end{array}$ & $\begin{array}{c}\mathrm{pH} \\
\text { standard } \\
\text { units }\end{array}$ & $\begin{array}{c}\text { Specific } \\
\text { conductance in } \\
\mathrm{mS} / \mathrm{cm} @ 25^{\circ} \mathrm{C}\end{array}$ & $\begin{array}{c}\text { Water } \\
\text { temperature, } \\
\text { degrees C }\end{array}$ \\
\hline \multirow[t]{10}{*}{423133083400101 Kent Lake near New Hudson, Mi. } & 04-09-2002 & 1031 & 3 & 12.6 & 8.1 & 732 & 6.5 \\
\hline & & 1032 & 6 & 12.6 & 8.1 & 731 & 6.5 \\
\hline & & 1033 & 9 & 12.8 & 8.1 & 731 & 6.5 \\
\hline & & 1034 & 12 & 12.7 & 8.2 & 730 & 6.5 \\
\hline & & 1035 & 15 & 12.8 & 8.2 & 730 & 6.5 \\
\hline & & 1036 & 18 & 12.6 & 8.2 & 730 & 6.5 \\
\hline & & 1037 & 21 & 12.8 & 8.2 & 730 & 6.0 \\
\hline & & 1038 & 24 & 12.6 & 8.2 & 730 & 6.0 \\
\hline & & 1039 & 27 & 12.7 & 8.2 & 731 & 6.0 \\
\hline & & 1040 & 30 & 12.7 & 8.2 & 733 & 6.0 \\
\hline \multirow[t]{11}{*}{423133083400101 Kent Lake near New Hudson, Mi. } & 08-07-2002 & 905 & 3 & 7.4 & 8.0 & 688 & 25.5 \\
\hline & & 906 & 6 & 7.4 & 8.0 & 688 & 25.5 \\
\hline & & 907 & 9 & 7.2 & 8.0 & 688 & 25.5 \\
\hline & & 909 & 12 & 6.9 & 8.0 & 688 & 25.5 \\
\hline & & 910 & 15 & 1.2 & 7.5 & 705 & 24.5 \\
\hline & & 912 & 18 & 1.0 & 7.4 & 711 & 21.0 \\
\hline & & 914 & 21 & 1.0 & 7.4 & 731 & 16.5 \\
\hline & & 916 & 24 & 1.0 & 7.4 & 739 & 14.0 \\
\hline & & 917 & 27 & 1.0 & 7.4 & 753 & 11.5 \\
\hline & & 918 & 30 & 1.0 & 7.4 & 764 & 10.0 \\
\hline & & 919 & 33 & 1.0 & 7.3 & 776 & 9.5 \\
\hline \multirow[t]{12}{*}{423332083290301 Wolverine Lake near Oakley Park, Mi. } & 09-08-2003 & 1400 & 3 & 8.7 & 8.1 & 987 & 22.9 \\
\hline & & 1401 & 8 & 8.3 & 8.1 & 990 & 22.6 \\
\hline & & 1402 & 13 & 8.7 & 8.1 & 995 & 22.3 \\
\hline & & 1403 & 18 & 7.3 & 7.5 & 1080 & 20.7 \\
\hline & & 1405 & 23 & 3.2 & 7.2 & 1080 & 17.7 \\
\hline & & 1406 & 28 & -- & 7.3 & 1030 & 13.2 \\
\hline & & 1408 & 33 & 3.4 & 7.4 & 1010 & 10.5 \\
\hline & & 1409 & 38 & 2.5 & 7.4 & 1000 & 8.2 \\
\hline & & 1410 & 43 & 2.5 & 7.4 & 1000 & 7.1 \\
\hline & & 1411 & 48 & 2.6 & 7.4 & 1000 & 6.8 \\
\hline & & 1412 & 53 & 3.0 & 7.4 & 1000 & 6.5 \\
\hline & & 1413 & 57 & 2.3 & 7.4 & 1010 & 6.4 \\
\hline \multirow{10}{*}{$\begin{array}{l}423607083252601 \text { Union Lake at Union Lake, Mi. } \\
\text { (Southeast basin) }\end{array}$} & 04-09-2002 & 1450 & 3 & 13.6 & 8.2 & 675 & 4.5 \\
\hline & & 1451 & 6 & 13.5 & 8.2 & 675 & 4.5 \\
\hline & & 1452 & 10 & 13.3 & 8.2 & 675 & 4.5 \\
\hline & & 1453 & 15 & 13.3 & 8.2 & 676 & 4.5 \\
\hline & & 1454 & 20 & 13.3 & 8.2 & 676 & 4.5 \\
\hline & & 1455 & 25 & 13.2 & 8.2 & 676 & 4.5 \\
\hline & & 1456 & 30 & 13.2 & 8.2 & 676 & 4.5 \\
\hline & & 1457 & 35 & 13.2 & 8.2 & 676 & 4.5 \\
\hline & & 1458 & 40 & 13.2 & 8.2 & 676 & 4.5 \\
\hline & & 1459 & 45 & 13.2 & 8.2 & 677 & 4.5 \\
\hline \multirow{14}{*}{$\begin{array}{l}423607083252601 \text { Union Lake at Union Lake, Mi. } \\
\text { (Southeast basin) }\end{array}$} & 08-06-2002 & 1311 & 3 & 6.8 & 8.4 & 672 & 26.0 \\
\hline & & 1313 & 9 & 6.7 & 8.4 & 673 & 26.0 \\
\hline & & 1314 & 15 & 6.7 & 8.3 & 673 & 25.0 \\
\hline & & 1316 & 21 & 7.0 & 8.0 & 669 & 18.5 \\
\hline & & 1317 & 27 & 6.4 & 7.9 & 671 & 14.5 \\
\hline & & 1318 & 30 & 4.8 & 7.7 & 675 & 13.5 \\
\hline & & 1319 & 33 & 3.9 & 7.6 & 676 & 13.0 \\
\hline & & 1321 & 36 & 3.3 & 7.6 & 678 & 12.5 \\
\hline & & 1322 & 39 & 2.4 & 7.5 & 681 & 12.0 \\
\hline & & 1323 & 42 & 1.8 & 7.5 & 683 & 11.2 \\
\hline & & 1324 & 45 & 1.7 & 7.5 & 685 & 10.6 \\
\hline & & 1325 & 48 & 1.6 & 7.5 & 688 & 10.0 \\
\hline & & 1326 & 54 & 1.6 & 7.4 & 699 & 9.5 \\
\hline & & 1327 & 57 & 1.6 & 7.4 & 702 & 9.0 \\
\hline
\end{tabular}


Table 11. Vertical profiles of field-measured physical characteristics of selected lakes in Oakland County, Michigan measured during 2002 and 2003 - cont'd. $\left[\mathrm{mg} / \mathrm{L}\right.$, milligrams per liter; $\mathrm{ms} / \mathrm{cm}$, microsiemens per centimeter $;{ }^{\circ} \mathrm{C}$, degrees Celsius]

\begin{tabular}{|c|c|c|c|c|c|c|c|c|}
\hline Station Number & Station Name & Sample Date & $\begin{array}{c}\text { Sample } \\
\text { Time }\end{array}$ & $\begin{array}{c}\text { Sampling } \\
\text { depth, in } \\
\text { feet }\end{array}$ & $\begin{array}{c}\text { Dissolved } \\
\text { oxygen in } \\
\mathrm{mg} / \mathrm{L} \\
\end{array}$ & $\begin{array}{c}\mathrm{pH} \\
\text { standard } \\
\text { units } \\
\end{array}$ & $\begin{array}{c}\text { Specific } \\
\text { conductance in } \\
\mathrm{mS} / \mathrm{cm} @ 25^{\circ} \mathrm{C}\end{array}$ & $\begin{array}{c}\text { Water } \\
\text { temperature, } \\
\text { degrees C } \\
\end{array}$ \\
\hline \multirow{16}{*}{423609083263001} & \multirow{16}{*}{$\begin{array}{l}\text { Union Lake at Union Lake, Mi. } \\
\text { (Southwest basin) }\end{array}$} & \multirow{16}{*}{ 04-29-2002 } & 1536 & 3 & 13.9 & 8.2 & 676 & 4.5 \\
\hline & & & 1537 & 6 & 13.3 & 8.2 & 677 & 4.5 \\
\hline & & & 1538 & 9 & 13.4 & 8.2 & 677 & 4.5 \\
\hline & & & 1539 & 12 & 13.4 & 8.2 & 677 & 4.5 \\
\hline & & & 1541 & 15 & 13.3 & 8.2 & 677 & 4.5 \\
\hline & & & 1542 & 18 & 13.2 & 8.2 & 677 & 4.5 \\
\hline & & & 1543 & 21 & 13.2 & 8.2 & 677 & 4.5 \\
\hline & & & 1544 & 25 & 13.2 & 8.2 & 677 & 4.5 \\
\hline & & & 1546 & 28 & 13.2 & 8.2 & 677 & 4.5 \\
\hline & & & 1547 & 32 & 13.2 & 8.2 & 677 & 4.5 \\
\hline & & & 1548 & 35 & 13.2 & 8.2 & 677 & 4.5 \\
\hline & & & 1549 & 40 & 13.2 & 8.2 & 677 & 4.5 \\
\hline & & & 1551 & 45 & 13.2 & 8.2 & 677 & 4.5 \\
\hline & & & 1552 & 50 & 13.2 & 8.2 & 677 & 4.5 \\
\hline & & & 1553 & 55 & 13.2 & 8.2 & 677 & 4.5 \\
\hline & & & 1554 & 60 & 13.0 & 8.2 & 677 & 4.5 \\
\hline \multirow{15}{*}{423609083263001} & \multirow{15}{*}{$\begin{array}{l}\text { Union Lake at Union Lake, Mi. } \\
\text { (Southwest basin) }\end{array}$} & \multirow[t]{15}{*}{ 08-06-2002 } & 1355 & 3 & 6.3 & 8.3 & 676 & 26.0 \\
\hline & & & 1357 & 9 & 6.1 & 8.3 & 675 & 25.5 \\
\hline & & & 1358 & 15 & 6.1 & 8.3 & 676 & 25.0 \\
\hline & & & 1359 & 24 & 5.3 & 7.7 & 675 & 15.5 \\
\hline & & & 1400 & 27 & 4.8 & 7.7 & 676 & 14.0 \\
\hline & & & 1401 & 30 & 4.8 & 7.7 & 675 & 13.0 \\
\hline & & & 1402 & 33 & 4.5 & 7.7 & 677 & 12.0 \\
\hline & & & 1403 & 36 & 4.5 & 7.7 & 679 & 11.0 \\
\hline & & & 1404 & 39 & 4.8 & 7.7 & 680 & 10.0 \\
\hline & & & 1406 & 42 & 5.3 & 7.7 & 682 & 9.0 \\
\hline & & & 1407 & 45 & 5.5 & 7.7 & 682 & 8.5 \\
\hline & & & 1409 & 48 & 5.6 & 7.7 & 685 & 7.0 \\
\hline & & & 1410 & 51 & 5.8 & 7.7 & 688 & 7.0 \\
\hline & & & 1411 & 57 & 4.6 & 7.6 & 693 & 6.5 \\
\hline & & & 1412 & 61 & 4.0 & 7.5 & 695 & 6.5 \\
\hline \multirow{25}{*}{423626083260201} & \multirow{25}{*}{$\begin{array}{l}\text { Union Lake at Union Lake, Mi. } \\
\text { (Central basin) }\end{array}$} & \multirow[t]{25}{*}{ 04-09-2002 } & 1310 & 3 & 14.0 & 8.3 & 674 & 4.0 \\
\hline & & & 1311 & 6 & 13.7 & 8.2 & 676 & 4.0 \\
\hline & & & 1312 & 9 & 13.6 & 8.2 & 676 & 4.0 \\
\hline & & & 1313 & 12 & 13.8 & 8.2 & 676 & 4.0 \\
\hline & & & 1314 & 15 & 13.6 & 8.2 & 676 & 4.0 \\
\hline & & & 1316 & 18 & 13.6 & 8.2 & 677 & 4.0 \\
\hline & & & 1317 & 21 & 13.5 & 8.2 & 676 & 4.0 \\
\hline & & & 1321 & 35 & 13.5 & 8.2 & 677 & 4.0 \\
\hline & & & 1322 & 38 & 13.5 & 8.2 & 677 & 4.0 \\
\hline & & & 1323 & 41 & 13.5 & 8.2 & 677 & 4.0 \\
\hline & & & 1324 & 44 & 13.5 & 8.2 & 677 & 4.0 \\
\hline & & & 1326 & 48 & 13.5 & 8.2 & 677 & 4.0 \\
\hline & & & 1328 & 52 & 13.5 & 8.2 & 677 & 4.0 \\
\hline & & & 1329 & 55 & 13.5 & 8.2 & 677 & 4.0 \\
\hline & & & 1331 & 60 & 13.5 & 8.2 & 677 & 4.0 \\
\hline & & & 1332 & 63 & 13.5 & 8.2 & 677 & 4.0 \\
\hline & & & 1333 & 67 & 13.5 & 8.2 & 677 & 4.0 \\
\hline & & & 1334 & 70 & 13.5 & 8.2 & 678 & 4.0 \\
\hline & & & 1335 & 75 & 13.5 & 8.2 & 678 & 4.0 \\
\hline & & & 1336 & 78 & 13.5 & 8.2 & 677 & 4.0 \\
\hline & & & 1337 & 82 & 13.4 & 8.2 & 677 & 4.0 \\
\hline & & & 1338 & 86 & 13.5 & 8.2 & 677 & 4.0 \\
\hline & & & 1339 & 90 & 13.4 & 8.2 & 678 & 4.1 \\
\hline & & & 1340 & 93 & 13.4 & 8.2 & 677 & 4.0 \\
\hline & & & 1341 & 96 & 13.1 & 8.2 & 678 & 4.0 \\
\hline
\end{tabular}


Table 11. Vertical profiles of field-measured physical characteristics of selected lakes in Oakland County, Michigan measured during 2002 and 2003 - cont'd. $\left[\mathrm{mg} / \mathrm{L}\right.$, milligrams per liter; $\mathrm{ms} / \mathrm{cm}$, microsiemens per centimeter $;{ }^{\circ} \mathrm{C}$, degrees Celsius]

\begin{tabular}{|c|c|c|c|c|c|c|c|}
\hline Station Number $\quad$ Station Name & Sample Date & $\begin{array}{c}\text { Sample } \\
\text { Time }\end{array}$ & $\begin{array}{c}\text { Sampling } \\
\text { depth, in } \\
\text { feet }\end{array}$ & $\begin{array}{c}\text { Dissolved } \\
\text { oxygen in } \\
\mathrm{mg} / \mathrm{L}\end{array}$ & $\begin{array}{c}\mathrm{pH} \\
\text { standard } \\
\text { units }\end{array}$ & $\begin{array}{c}\text { Specific } \\
\text { conductance in } \\
\mathrm{mS} / \mathrm{cm} @ 25^{\circ} \mathrm{C}\end{array}$ & $\begin{array}{c}\text { Water } \\
\text { temperature, } \\
\text { degrees C }\end{array}$ \\
\hline \multirow{18}{*}{$\begin{array}{c}423626083260201 \text { Union Lake at Union Lake, Mi. } \\
\text { (Central basin) }\end{array}$} & \multirow[t]{18}{*}{$08-06-2002$} & 1222 & 3 & 7.4 & 8.3 & 673 & 26.5 \\
\hline & & 1223 & 10 & 7.2 & 8.3 & 673 & 26.5 \\
\hline & & 1226 & 20 & 8.4 & 8.2 & 665 & 21.5 \\
\hline & & 1227 & 30 & 7.7 & 7.9 & 669 & 14.5 \\
\hline & & 1228 & 33 & 7.2 & 7.9 & 671 & 12.5 \\
\hline & & 1229 & 36 & 7.0 & 7.9 & 672 & 12.0 \\
\hline & & 1231 & 39 & 6.8 & 7.8 & 673 & 11.5 \\
\hline & & 1232 & 42 & 6.6 & 7.8 & 674 & 10.5 \\
\hline & & 1233 & 45 & 6.7 & 7.8 & 675 & 10.0 \\
\hline & & 1234 & 48 & 6.9 & 7.8 & 676 & 9.0 \\
\hline & & 1235 & 51 & 7.1 & 7.8 & 676 & 9.0 \\
\hline & & 1236 & 54 & 7.0 & 7.8 & 677 & 8.5 \\
\hline & & 1237 & 57 & 7.0 & 7.8 & 677 & 8.0 \\
\hline & & 1238 & 60 & 7.0 & 7.8 & 678 & 7.5 \\
\hline & & 1239 & 70 & 6.9 & 7.7 & 679 & 7.0 \\
\hline & & 1241 & 80 & 6.8 & 7.7 & 680 & 6.5 \\
\hline & & 1242 & 90 & 6.3 & 7.7 & 681 & 6.0 \\
\hline & & 1243 & 100 & 5.7 & 7.6 & 683 & 6.0 \\
\hline \multirow[t]{10}{*}{423730083363401 Lower Pettibone Lake near Highland, Mi. } & \multirow[t]{10}{*}{ 04-17-2002 } & 852 & 3 & 10.2 & 8.2 & 683 & 16.5 \\
\hline & & 853 & 7 & 11.5 & 8.2 & 684 & 12.0 \\
\hline & & 854 & 11 & 12.7 & 8.2 & 690 & 8.5 \\
\hline & & 856 & 15 & 12.5 & 8.1 & 693 & 7.5 \\
\hline & & 857 & 19 & 12.3 & 8.1 & 694 & 6.5 \\
\hline & & 858 & 23 & 12.0 & 8.0 & 695 & 6.0 \\
\hline & & 859 & 27 & 11.7 & 8.0 & 695 & 6.0 \\
\hline & & 901 & 31 & 11.4 & 8.0 & 695 & 5.5 \\
\hline & & 902 & 35 & 11.3 & 7.9 & 696 & 5.5 \\
\hline & & 903 & 39 & 6.3 & 7.8 & 728 & 5.5 \\
\hline \multirow[t]{12}{*}{423730083363401 Lower Pettibone Lake near Highland, Mi. } & \multirow[t]{12}{*}{ 08-06-2002 } & 840 & 3 & 7.3 & 7.4 & 627 & 26.0 \\
\hline & & 841 & 6 & 7.7 & 8.1 & 628 & 26.0 \\
\hline & & 842 & 9 & 7.6 & 8.1 & 627 & 26.0 \\
\hline & & 843 & 12 & 7.8 & 7.8 & 648 & 25.0 \\
\hline & & 844 & 15 & 11.7 & 7.8 & 629 & 22.0 \\
\hline & & 846 & 18 & 12.8 & 7.9 & 626 & 18.0 \\
\hline & & 847 & 21 & 12.9 & 8.0 & 637 & 14.5 \\
\hline & & 849 & 24 & 12.6 & 8.0 & 654 & 12.0 \\
\hline & & 851 & 27 & 10.1 & 7.8 & 676 & 9.5 \\
\hline & & 852 & 30 & 3.6 & 7.5 & 684 & 8.5 \\
\hline & & 854 & 33 & 2.2 & 7.4 & 690 & 7.5 \\
\hline & & 856 & 36 & 1.8 & 7.4 & 703 & 7.0 \\
\hline \multirow[t]{9}{*}{424306083314201 Big Lake at Andersonville, Mi. } & \multirow[t]{9}{*}{$04-15-2002$} & 1437 & 0.5 & 11.3 & 8.3 & 329 & 16.0 \\
\hline & & 1438 & 1 & 11.2 & 8.3 & 329 & 16.0 \\
\hline & & 1439 & 1.5 & 11.1 & 8.3 & 330 & 16.0 \\
\hline & & 1441 & 2 & 11.1 & 8.4 & 329 & 15.5 \\
\hline & & 1442 & 2.5 & 11.1 & 8.4 & 330 & 15.5 \\
\hline & & 1443 & 3 & 11.2 & 8.4 & 329 & 15.5 \\
\hline & & 1444 & 3.5 & 11.3 & 8.4 & 329 & 15.5 \\
\hline & & 1446 & 4 & 11.3 & 8.4 & 330 & 15.0 \\
\hline & & 1448 & 4.5 & 15.0 & 7.6 & 405 & 15.0 \\
\hline \multirow[t]{8}{*}{424306083314201 Big Lake at Andersonville, Mi. } & \multirow[t]{8}{*}{ 08-07-2002 } & 1424 & 0.3 & 8.9 & 8.5 & 292 & 24.0 \\
\hline & & 1425 & 0.6 & 8.7 & 8.5 & 292 & 24.0 \\
\hline & & 1426 & 0.9 & 8.6 & 8.5 & 292 & 24.5 \\
\hline & & 1427 & 1.2 & 8.4 & 8.5 & 292 & 24.4 \\
\hline & & 1429 & 1.5 & 8.2 & 8.5 & 292 & 24.5 \\
\hline & & 1430 & 1.8 & 8.2 & 8.5 & 293 & 24.5 \\
\hline & & 1431 & 2.1 & 7.7 & 8.4 & 293 & 24.5 \\
\hline & & 1432 & 2.4 & 3.3 & 8.0 & 298 & 24.5 \\
\hline
\end{tabular}


Table 11. Vertical profiles of field-measured physical characteristics of selected lakes in Oakland County, Michigan measured during 2002 and 2003 - cont'd. $\left[\mathrm{mg} / \mathrm{L}\right.$, milligrams per liter; $\mathrm{ms} / \mathrm{cm}$, microsiemens per centimeter $;{ }^{\circ} \mathrm{C}$, degrees Celsius]

\begin{tabular}{|c|c|c|c|c|c|c|c|}
\hline Station Number $\quad$ Station Name & Sample Date & $\begin{array}{c}\text { Sample } \\
\text { Time }\end{array}$ & $\begin{array}{c}\text { Sampling } \\
\text { depth, in } \\
\text { feet }\end{array}$ & $\begin{array}{c}\text { Dissolved } \\
\text { oxygen in } \\
\mathrm{mg} / \mathrm{L}\end{array}$ & $\begin{array}{c}\mathrm{pH} \\
\text { standard } \\
\text { units }\end{array}$ & $\begin{array}{c}\text { Specific } \\
\text { conductance in } \\
\mathrm{mS} / \mathrm{cm} @ 25^{\circ} \mathrm{C}\end{array}$ & $\begin{array}{c}\text { Water } \\
\text { temperature, } \\
\text { degrees C }\end{array}$ \\
\hline \multirow[t]{10}{*}{424313083312001 Big Lake at Andersonville, Mi. } & $04-15-2002$ & 1402 & 3 & 10.8 & 8.3 & 333 & 15.0 \\
\hline & & 1403 & 4 & 10.9 & 8.3 & 333 & 15.0 \\
\hline & & 1405 & 5 & 10.9 & 8.3 & 333 & 15.0 \\
\hline & & 1406 & 6 & 10.9 & 8.3 & 333 & 15.0 \\
\hline & & 1407 & 7 & 10.6 & 8.3 & 334 & 14.0 \\
\hline & & 1408 & 8 & 10.6 & 8.2 & 335 & 12.0 \\
\hline & & 1409 & 9 & 10.7 & 8.2 & 336 & 12.0 \\
\hline & & 1410 & 10 & 10.7 & 8.2 & 335 & 11.5 \\
\hline & & 1411 & 11 & 10.6 & 8.1 & 336 & 11.5 \\
\hline & & 1412 & 12 & 10.4 & 7.9 & 359 & 11.5 \\
\hline \multirow[t]{11}{*}{424313083312001 Big Lake at Andersonville, Mi. } & 08-07-2002 & 1347 & 3 & 8.6 & 8.4 & 291 & 25.0 \\
\hline & & 1348 & 3.7 & 8.6 & 8.4 & 291 & 25.0 \\
\hline & & 1349 & 4.4 & 8.5 & 8.4 & 291 & 25.0 \\
\hline & & 1350 & 5.1 & 8.5 & 8.4 & 291 & 25.0 \\
\hline & & 1351 & 5.8 & 8.5 & 8.4 & 291 & 25.0 \\
\hline & & 1352 & 6.5 & 8.5 & 8.4 & 291 & 25.0 \\
\hline & & 1353 & 7.2 & 8.5 & 8.4 & 291 & 25.0 \\
\hline & & 1354 & 7.9 & 8.6 & 8.5 & 291 & 25.0 \\
\hline & & 1355 & 8.6 & 8.6 & 8.5 & 291 & 24.5 \\
\hline & & 1356 & 9.3 & 8.8 & 8.5 & 290 & 24.5 \\
\hline & & 1357 & 10 & 8.4 & 8.4 & 293 & 24.0 \\
\hline \multirow[t]{12}{*}{424335083405201 Tipsico Lake near Rose Center, Mi. } & $09-08-2003$ & 1025 & 3 & 8.2 & 8.0 & 194 & 22.6 \\
\hline & & 1026 & 4.5 & 8.1 & 8.0 & 194 & 22.6 \\
\hline & & 1027 & 6 & 8.0 & 8.0 & 193 & 22.6 \\
\hline & & 1028 & 7.5 & 8.2 & 8.0 & 193 & 22.5 \\
\hline & & 1029 & 9 & 8.2 & 8.1 & 194 & 22.5 \\
\hline & & 1030 & 10.5 & 8.0 & 8.0 & 194 & 22.3 \\
\hline & & 1031 & 12 & 7.7 & 8.0 & 194 & 22.1 \\
\hline & & 1032 & 13.5 & 7.2 & 7.8 & 194 & 21.9 \\
\hline & & 1033 & 15 & 6.3 & 7.6 & 194 & 21.8 \\
\hline & & 1034 & 16.5 & 5.5 & 7.3 & 195 & 21.7 \\
\hline & & 1035 & 18 & 4.2 & 7.3 & 195 & 21.6 \\
\hline & & 1036 & 19 & 2.4 & 7.0 & 225 & 21.4 \\
\hline \multirow[t]{11}{*}{424634083143601 Lake Orion near Lake Orion, Mi. } & 09-09-2003 & 735 & 3 & 7.8 & 7.7 & 545 & 22.7 \\
\hline & & 836 & 8 & 7.9 & 7.7 & 545 & 22.7 \\
\hline & & 837 & 13 & 7.8 & 7.7 & 545 & 22.6 \\
\hline & & 838 & 18 & 5.4 & 7.4 & 554 & 20.7 \\
\hline & & 839 & 23 & 7.1 & 7.3 & 560 & 14.6 \\
\hline & & 840 & 28 & 7.4 & 7.4 & 554 & 10.0 \\
\hline & & 841 & 33 & 6.3 & 7.3 & 553 & 7.4 \\
\hline & & 842 & 38 & 4.2 & 7.3 & 553 & 6.2 \\
\hline & & 843 & 43 & 1.9 & 7.2 & 557 & 5.5 \\
\hline & & 844 & 48 & 1.0 & 7.2 & 562 & 5.2 \\
\hline & & 845 & 53 & 0.8 & 7.3 & 571 & 5.1 \\
\hline \multirow[t]{12}{*}{424804083311301 Valley Lake near Davisburg, Mi. } & 09-10-2003 & 815 & 3 & 8.2 & 7.7 & 388 & 22.3 \\
\hline & & 817 & 7 & 7.7 & 7.7 & 389 & 22.3 \\
\hline & & 819 & 11 & 7.2 & 7.6 & 389 & 21.9 \\
\hline & & 821 & 15 & 6.6 & 7.5 & 400 & 20.4 \\
\hline & & 823 & 19 & 8.9 & 7.5 & 436 & 14.0 \\
\hline & & 825 & 23 & 2.9 & 7.3 & 449 & 10.3 \\
\hline & & 827 & 27 & 1.2 & 7.2 & 472 & 7.8 \\
\hline & & 829 & 31 & 0.8 & 7.2 & 498 & 6.3 \\
\hline & & 831 & 35 & 0.8 & 7.2 & 506 & 6.0 \\
\hline & & 833 & 39 & 0.7 & 7.2 & 529 & 5.6 \\
\hline & & 835 & 43 & 0.6 & 7.0 & 543 & 5.4 \\
\hline & & 837 & 44 & 0.6 & 7.0 & 557 & 5.5 \\
\hline
\end{tabular}


Table 11. Vertical profiles of field-measured physical characteristics of selected lakes in Oakland County, Michigan measured during 2002 and 2003 - cont'd. $\left[\mathrm{mg} / \mathrm{L}\right.$, milligrams per liter; $\mathrm{ms} / \mathrm{cm}$, microsiemens per centimeter $;{ }^{\circ} \mathrm{C}$, degrees Celsius]

\begin{tabular}{|c|c|c|c|c|c|c|c|}
\hline Station Number $\quad$ Station Name & Sample Date & $\begin{array}{c}\text { Sample } \\
\text { Time }\end{array}$ & $\begin{array}{c}\text { Sampling } \\
\text { depth, in } \\
\text { feet }\end{array}$ & $\begin{array}{c}\text { Dissolved } \\
\text { oxygen in } \\
\mathrm{mg} / \mathrm{L}\end{array}$ & $\begin{array}{c}\mathrm{pH} \\
\text { standard } \\
\text { units }\end{array}$ & $\begin{array}{c}\text { Specific } \\
\text { conductance in } \\
\mathrm{mS} / \mathrm{cm} @ 25^{\circ} \mathrm{C}\end{array}$ & $\begin{array}{c}\text { Water } \\
\text { temperature, } \\
\text { degrees C }\end{array}$ \\
\hline \multirow[t]{13}{*}{424828083400201 Dickinson Lake near Holly, Mi. } & $09-10-2003$ & 1220 & 3 & 8.3 & 7.8 & 505 & 23.0 \\
\hline & & 1222 & 8 & 8.2 & 7.8 & 502 & 22.9 \\
\hline & & 1224 & 13 & 8.3 & 7.8 & 503 & 22.8 \\
\hline & & 1226 & 18 & 9.8 & 7.8 & 504 & 21.4 \\
\hline & & 1228 & 23 & 11.1 & 7.8 & 512 & 13.1 \\
\hline & & 1230 & 28 & 9.0 & 7.7 & 514 & 9.1 \\
\hline & & 1232 & 33 & 4.8 & 7.5 & 519 & 6.9 \\
\hline & & 1234 & 38 & 1.6 & 7.3 & 524 & 5.8 \\
\hline & & 1236 & 43 & 1.0 & 7.4 & 523 & 5.8 \\
\hline & & 1238 & 48 & 0.9 & 7.4 & 525 & 5.7 \\
\hline & & 1240 & 53 & 0.9 & 7.4 & 528 & 5.5 \\
\hline & & 1242 & 58 & 0.8 & 7.4 & 529 & 5.5 \\
\hline & & 1244 & 63 & 0.8 & 7.3 & 534 & 5.5 \\
\hline \multirow[t]{13}{*}{424830083311801 Heron Lake near Holly, Mi. } & $04-15-2003$ & 1042 & 3 & 13.3 & 8.1 & 400 & 9.5 \\
\hline & & 1044 & 6 & 12.3 & 8.1 & 401 & 9.0 \\
\hline & & 1045 & 9 & 12.2 & 8.1 & 400 & 9.0 \\
\hline & & 1046 & 12 & 12.1 & 8.1 & 400 & 9.0 \\
\hline & & 1047 & 15 & 12.1 & 8.1 & 400 & 9.0 \\
\hline & & 1049 & 18 & 12.2 & 8.1 & 400 & 8.5 \\
\hline & & 1051 & 21 & 12.2 & 8.1 & 400 & 8.0 \\
\hline & & 1052 & 24 & 11.9 & 8.0 & 400 & 4.5 \\
\hline & & 1053 & 27 & 11.8 & 8.0 & 401 & 4.5 \\
\hline & & 1054 & 30 & 11.5 & 7.9 & 402 & 4.5 \\
\hline & & 1055 & 33 & 11.4 & 7.9 & 401 & 4.5 \\
\hline & & 1056 & 36 & 11.1 & 7.9 & 402 & 4.5 \\
\hline & & 1057 & 39 & 10.5 & 7.8 & 416 & 4.5 \\
\hline \multirow[t]{10}{*}{424830083311801 Heron Lake near Holly, Mi. } & $08-21-2003$ & 1141 & 3 & 9.1 & & 371 & 26.0 \\
\hline & & 1143 & 7 & 9.2 & & 371 & 26.0 \\
\hline & & 1145 & 11 & 8.6 & & 372 & 26.0 \\
\hline & & 1147 & 15 & 0.7 & & 443 & 24.0 \\
\hline & & 1149 & 19 & 0.3 & & 417 & 14.5 \\
\hline & & 1152 & 23 & 0.3 & & 455 & 14.5 \\
\hline & & 1158 & 25 & 0.5 & & 497 & 6.5 \\
\hline & & 1154 & 27 & 0.3 & & 463 & 11.0 \\
\hline & & 1156 & 31 & 0.3 & & 471 & 8.0 \\
\hline & & 1200 & 38 & 0.6 & & 586 & 6.5 \\
\hline \multirow[t]{12}{*}{424830083311801 Heron Lake near Holly, Mi. } & $09-10-2003$ & 1025 & 3 & 7.4 & 8.1 & 420 & 22.5 \\
\hline & & 1027 & 6 & 7.4 & 8.1 & 420 & 22.5 \\
\hline & & 1029 & 9 & 7.3 & 8.1 & 420 & 22.5 \\
\hline & & 1031 & 12 & 6.8 & 8.1 & 421 & 22.5 \\
\hline & & 1033 & 15 & 4.3 & 7.7 & 426 & 22.5 \\
\hline & & 1035 & 18 & 1.2 & 7.2 & 434 & 22.5 \\
\hline & & 1037 & 21 & 0.6 & 6.9 & 500 & 18.5 \\
\hline & & 1039 & 24 & 0.6 & 7.0 & 485 & 15.0 \\
\hline & & 1041 & 28 & 0.5 & 7.1 & 488 & 11.0 \\
\hline & & 1043 & 32 & 0.5 & 7.1 & 491 & 8.5 \\
\hline & & 1045 & 36 & 0.5 & 7.2 & 523 & 7.0 \\
\hline & & 1047 & 40 & 0.5 & 6.9 & 587 & 6.5 \\
\hline \multirow[t]{13}{*}{424952083084301 Lakeville Lake near Lakeville, Mi. } & 09-09-2003 & 1205 & 3 & 9.0 & 8.3 & 383 & 22.8 \\
\hline & & 1206 & 8 & 8.3 & 6.3 & 384 & 22.4 \\
\hline & & 1208 & 13 & 7.7 & 8.2 & 388 & 21.8 \\
\hline & & 1212 & 16 & 5.5 & 7.3 & 485 & 20.0 \\
\hline & & 1210 & 18 & 6.7 & 7.3 & 488 & 17.1 \\
\hline & & 1215 & 23 & 8.2 & 7.4 & 490 & 11.4 \\
\hline & & 1217 & 28 & 5.4 & 7.3 & 514 & 8.3 \\
\hline & & 1219 & 33 & 1.5 & 7.2 & 530 & 6.8 \\
\hline & & 1221 & 38 & 0.9 & 7.3 & 535 & 5.7 \\
\hline & & 1223 & 43 & 0.7 & 7.3 & 543 & 5.3 \\
\hline & & 1225 & 48 & 0.6 & 7.3 & 544 & 5.1 \\
\hline & & 1227 & 53 & 0.6 & 7.2 & 554 & 4.8 \\
\hline & & 1229 & 55.5 & 0.6 & 7.2 & 560 & 4.8 \\
\hline
\end{tabular}


Table 12. Results of chemical water-quality analyses for selected parameters at selected lakes in Oakland Ccounty, Michigan, 2002 - 2003 $\left[\mu \mathrm{s} / \mathrm{cm}\right.$, microsiemens per centimeter @ $25^{\circ} \mathrm{C} ; \mathrm{mg} / \mathrm{L}$, milligrams per liter; $\mu \mathrm{g} / \mathrm{L}$, micrograms per liter $]$

\begin{tabular}{|c|c|c|c|c|c|c|c|c|c|c|c|c|c|}
\hline Site Number & Lake Name & Sample Date & Position in water column & $\begin{array}{c}\text { Time } \\
\text { sampled }\end{array}$ & $\begin{array}{c}\text { Sampling } \\
\text { depth in } \\
\text { feet }\end{array}$ & 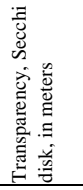 & 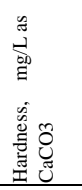 & 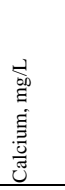 & 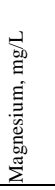 & 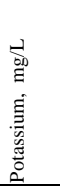 & 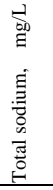 & 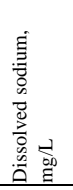 & 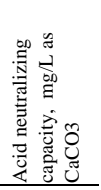 \\
\hline \multirow[t]{4}{*}{423133083400105} & Kent Lake near New Hudson & 04-09-2002 & 3 feet below surface & 1045 & 3 & 2.4 & & & & & & & \\
\hline & & & Mid-level/thermocline & 1050 & 15 & & 240 & 63.5 & 20.2 & 2.4 & 60 & & 181 \\
\hline & & & Whole water column & 1015 & 16 & & & & & & & & \\
\hline & & & $3 \mathrm{ft}$ off bottom & 1055 & 27 & & & & & & & & \\
\hline \multirow[t]{4}{*}{423133083400105} & Kent Lake near New Hudson & 08-07-2002 & 3 feet below surface & 920 & 3 & 1.4 & & & & & & & \\
\hline & & & Whole water column & 855 & 9 & & & & & & & & \\
\hline & & & Mid-level/thermocline & 925 & 17 & & & & & & & & \\
\hline & & & $3 \mathrm{ft}$ off bottom & 930 & 33 & & & & & & & & \\
\hline \multirow[t]{4}{*}{423332083290305} & Wolverine Lake & 09-08-2003 & 3 feet below surface & 1415 & 3 & 6.1 & & & & & & & \\
\hline & near Oakley Park & & Mid-level/thermocline & 1420 & 20 & & & 54.6 & 23.1 & 1.9 & & 147 & \\
\hline & & & Whole water column & 1410 & 40 & & & & & & & & \\
\hline & & & $3 \mathrm{ft}$ off bottom & 1425 & 55 & & & & & & & & \\
\hline \multirow[t]{4}{*}{423607083252605} & Union Lake at Union Lake & 04-09-2002 & 3 feet below surface & 1510 & 3 & 6.7 & & & & & & & \\
\hline & & & Whole water column & 1515 & 25 & & 200 & 46.6 & 21.1 & 2.4 & 60 & & 144 \\
\hline & & & Mid-level/thermocline & 1445 & 44 & & & & & & & & \\
\hline & & & $3 \mathrm{ft}$ off bottom & 1525 & 48 & & & & & & & & \\
\hline \multirow[t]{4}{*}{423607083252605} & Union Lake at Union Lake & 08-06-2002 & 3 feet below surface & 1330 & 3 & 3.4 & & & & & & & \\
\hline & & & Whole water column & 1315 & 22 & & & & & & & & \\
\hline & & & Mid-level/thermocline & 1335 & 33 & & & & & & & & \\
\hline & & & $3 \mathrm{ft}$ off bottom & 1340 & 57 & & & & & & & & \\
\hline \multirow[t]{4}{*}{423609083263005} & Union Lake at Union Lake & 04-09-2002 & 3 feet below surface & 1600 & 3 & 7.9 & & & & & & & \\
\hline & & & Whole water column & 1605 & 30 & & 200 & 46 & 20.4 & 2.3 & 59 & & 144 \\
\hline & & & Mid-level/thermocline & 1530 & 52 & & & & & & & & \\
\hline & & & $3 \mathrm{ft}$ off bottom & 1610 & 57 & & & & & & & & \\
\hline \multirow[t]{4}{*}{423609083263005} & Union Lake at Union Lake & 08-06-2002 & 3 feet below surface & 1420 & 3 & 2.7 & & & & & & & \\
\hline & & & Whole water column & 1345 & 18 & & & & & & & & \\
\hline & & & Mid-level/thermocline & 1425 & 35 & & & & & & & & \\
\hline & & & $3 \mathrm{ft}$ off bottom & 1430 & 62 & & & & & & & & \\
\hline \multirow[t]{4}{*}{423626083260205} & Union Lake at Union Lake & 04-09-2002 & 3 feet below surface & 1345 & 3 & 8.5 & & & & & & & \\
\hline & & & Mid-level/thermocline & 1350 & 50 & & 200 & 47.2 & 20.9 & 2.5 & 61 & & 143 \\
\hline & & & Whole water column & 1315 & 56 & & & & & & & & \\
\hline & & & $3 \mathrm{ft}$ off bottom & 1355 & 98 & & & & & & & & \\
\hline \multirow[t]{4}{*}{423626083260205} & Union Lake at Union Lake & 08-06-2002 & 3 feet below surface & 1245 & 3 & 3.4 & & & & & & & \\
\hline & & & Whole water column & 1225 & 22 & & & & & & & & \\
\hline & & & Mid-level/thermocline & 1250 & 50 & & & & & & & & \\
\hline & & & $3 \mathrm{ft}$ off bottom & 1255 & 106 & & & & & & & & \\
\hline \multirow[t]{4}{*}{423730083363405} & Lower Pettibone Lake & 04-17-2002 & 3 feet below surface & 850 & 3 & 3 & & & & & & & \\
\hline & near Highland & & Whole water column & 845 & 20 & & & & & & & & \\
\hline & & & Mid-level/thermocline & 855 & 20 & & 250 & 66 & 20.6 & 1.6 & 50 & & 199 \\
\hline & & & $3 \mathrm{ft}$ off bottom & 900 & 36 & & & & & & & & \\
\hline \multirow[t]{4}{*}{423730083363405} & Lower Pettibone Lake & 08-06-2002 & 3 feet below surface & 900 & 3 & 4.3 & & & & & & & \\
\hline & near Highland & & Mid-level/thermocline & 905 & 21 & & & & & & & & \\
\hline & & & Whole water column & 840 & 28 & & & & & & & & \\
\hline & & & $3 \mathrm{ft}$ off bottom & 910 & 36 & & & & & & & & \\
\hline \multirow[t]{3}{*}{424306083314206} & Big Lake at Andersonville & $04-15-2002$ & Mid-level/thermocline & 1455 & 3 & & 140 & 40.8 & 9.5 & 1.3 & 12 & & 95 \\
\hline & & & Whole water column & 1440 & 4 & & & & & & & & \\
\hline & & & 3 feet below surface & 1450 & & 1.5 & & & & & & & \\
\hline \multirow[t]{3}{*}{424306083314205} & Big Lake at Andersonville & 08-07-2002 & 3 feet below surface & 1455 & 2 & 0.9 & & & & & & & \\
\hline & & & Mid-level/thermocline & 1455 & 2 & & & & & & & & \\
\hline & & & Whole water column & 1425 & 3 & & & & & & & & \\
\hline 424313083312005 & Big Lake at Andersonville & $04-15-2002$ & 3 feet below surface & 1400 & 3 & 2.7 & & & & & & & \\
\hline & & & Mid-level/thermocline & 1405 & 6 & & 140 & 39.3 & 9.1 & 1.2 & 10 & & 95 \\
\hline & & & $3 \mathrm{ft}$ off bottom & 1410 & 10 & & & & & & & & \\
\hline & & & Whole water column & 1350 & 10 & & & & & & & & \\
\hline 424313083312005 & Big Lake at Andersonville & 08-07-2002 & 3 feet below surface & 1405 & 3 & 1.1 & & & & & & & \\
\hline & & & Whole water column & 1400 & 7 & & & & & & & & \\
\hline & & & Mid-level/thermocline & 1410 & 7 & & & & & & & & \\
\hline & & & $3 \mathrm{ft}$ off bottom & 1415 & 10 & & & & & & & & \\
\hline
\end{tabular}


Table 12. Results of chemical water-quality analyses for selected parameters at selected lakes in Oakland Ccounty, Michigan, 2002 - 2003, continued $\left[\mu \mathrm{s} / \mathrm{cm}\right.$, microsiemens per centimeter @ $25^{\circ} \mathrm{C} ; \mathrm{mg} / \mathrm{L}$, milligrams per liter; $\mu \mathrm{g} / \mathrm{L}$, micrograms per liter]

\begin{tabular}{|c|c|c|c|c|c|c|c|c|c|c|c|c|}
\hline Site Number & Lake Name & Sample Date & Position in water column & 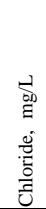 & 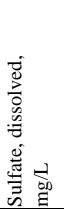 & 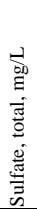 & 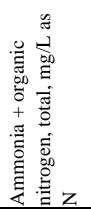 & 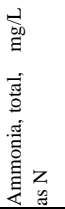 & 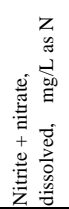 & 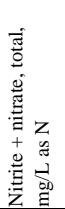 & 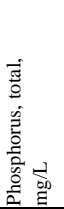 & 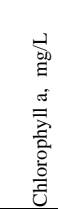 \\
\hline \multirow[t]{3}{*}{423133083400105} & Kent Lake near New Hudson & $04-09-2002$ & 3 feet below surface & & & & 0.17 & 0.03 & & 0.145 & 0.02 & \\
\hline & & & Mid-level/thermocline & 117 & & 25 & 0.22 & 0.03 & & 0.144 & 0.02 & \\
\hline & & & $\begin{array}{l}\text { Whole water column } \\
3 \mathrm{ft} \text { off bottom }\end{array}$ & & & & 0.21 & 0.03 & & 0.142 & 0.02 & \\
\hline \multirow[t]{3}{*}{423133083400105} & Kent Lake near New Hudson & 08-07-2002 & 3 feet below surface & & & & 0.45 & 0.03 & & $<.010$ & 0.03 & \\
\hline & & & Whole water column & & & & & & & 010 & 00 & \\
\hline & & & $\begin{array}{l}\text { Mid-level/thermocline } \\
3 \mathrm{ft} \text { off bottom }\end{array}$ & & & & $\begin{array}{l}0.49 \\
0.89\end{array}$ & $\begin{array}{l}0.07 \\
0.63\end{array}$ & & $<.010$ & 0.04 & \\
\hline \multirow[t]{3}{*}{423332083290305} & Wolverine Lake & 09-08-2003 & 3 feet below surface & & & & 0.39 & $<.01$ & $<.022$ & & $<.04$ & \\
\hline & near Oakley Park & & $\begin{array}{l}\text { Mid-level/thermocline } \\
\text { Whole water column }\end{array}$ & 263 & 32.2 & & 0.41 & $<.01$ & $<.022$ & & $<.04$ & \\
\hline & & & $3 \mathrm{ft}$ off bottom & & & & 1.50 & 0.81 & $<.022$ & & E.04 & \\
\hline \multirow[t]{3}{*}{423607083252605} & Union Lake at Union Lake & 04-09-2002 & 3 feet below surface & & & & 0.09 & 0.05 & & 0.083 & 0.01 & \\
\hline & & & Whole water column & 118 & & 20 & 0.11 & 0.06 & & 0.084 & 0.01 & \\
\hline & & & $\begin{array}{l}\text { Mid-level/thermocline } \\
3 \mathrm{ft} \text { off bottom }\end{array}$ & & & & 0.11 & 0.05 & & 0.081 & 0.01 & \\
\hline \multirow[t]{3}{*}{423607083252605} & Union Lake at Union Lake & 08-06-2002 & $\begin{array}{l}3 \text { feet below surface } \\
\text { Whole water column }\end{array}$ & & & & 0.40 & 0.03 & & $<.010$ & 0.01 & \\
\hline & & & Mid-level/thermocline & & & & 0.32 & 0.13 & & 0.013 & 0.01 & \\
\hline & & & $3 \mathrm{ft}$ off bottom & & & & 1.30 & 0.96 & & $<.010$ & 0.03 & \\
\hline \multirow[t]{3}{*}{423609083263005} & Union Lake at Union Lake & 04-09-2002 & 3 feet below surface & & & & 0.15 & 0.05 & & 0.081 & 0.01 & \\
\hline & & & Whole water column & 119 & & 21 & $<.10$ & 0.05 & & 0.083 & 0.01 & \\
\hline & & & $\begin{array}{l}\text { Mid-level/thermocline } \\
3 \mathrm{ft} \text { off bottom }\end{array}$ & & & & $<.10$ & 0.05 & & 0.080 & 0.02 & \\
\hline \multirow[t]{4}{*}{423609083263005} & Union Lake at Union Lake & 08-06-2002 & 3 feet below surface & & & & 0.34 & 0.03 & & $<.010$ & 0.01 & \\
\hline & & & Whole water column & & & & & & & & & \\
\hline & & & Mid-level/thermocline & & & & 0.39 & 0.12 & & 0.012 & 0.01 & \\
\hline & & & $3 \mathrm{ft}$ off bottom & & & & 0.50 & 0.34 & & 0.032 & 0.01 & \\
\hline \multirow[t]{3}{*}{423626083260205} & Union Lake at Union Lake & 04-09-2002 & 3 feet below surface & & & & $<.10$ & 0.05 & & 0.087 & 0.01 & \\
\hline & & & Mid-level/thermocline & 119 & & 21 & 0.11 & 0.05 & & 0.082 & 0.01 & \\
\hline & & & $\begin{array}{l}\text { Whole water column } \\
3 \mathrm{ft} \text { off bottom }\end{array}$ & & & & $<10$ & 0.05 & & 0.083 & 0.01 & \\
\hline \multirow[t]{4}{*}{423626083260205} & Union Lake at Union Lake & 08-06-2002 & 3 feet below surface & & & & 0.32 & 0.04 & & $<.010$ & 0.01 & \\
\hline & & & Whole water column & & & & & & & & & \\
\hline & & & Mid-level/thermocline & & & & 0.38 & 0.10 & & 0.044 & 0.01 & \\
\hline & & & $3 \mathrm{ft}$ off bottom & & & & 0.40 & 0.22 & & 0.087 & 0.01 & \\
\hline \multirow[t]{3}{*}{423730083363405} & Lower Pettibone Lake & 04-17-2002 & 3 feet below surface & & & & 0.15 & 0.02 & & 0.327 & 0.01 & \\
\hline & & & Mid-level/thermocline & 96 & & 12 & $<.10$ & 0.01 & & 0.353 & 0.01 & \\
\hline & & & $3 \mathrm{ft}$ off bottom & & & & $<.10$ & 0.04 & & 0.346 & 0.01 & \\
\hline \multirow[t]{4}{*}{423730083363405} & Lower Pettibone Lake & 08-06-2002 & 3 feet below surface & & & & 0.18 & 0.04 & & 0.015 & 0.01 & \\
\hline & near Highland & & Mid-level/thermocline & & & & 0.17 & 0.07 & & $<.010$ & 0.01 & \\
\hline & & & Whole water column & & & & & & & & & \\
\hline & & & $3 \mathrm{ft}$ off bottom & & & & 0.53 & 0.23 & & 0.015 & 0.05 & \\
\hline \multirow[t]{2}{*}{424306083314206} & Big Lake at Andersonville & 04-15-2002 & Mid-level/thermocline & 32 & & 6 & 0.42 & 0.02 & & 0.168 & 0.02 & $<1.00$ \\
\hline & & & 3 feet below surface & & & & & & & & & 7 \\
\hline \multirow[t]{3}{*}{424306083314205} & Big Lake at Andersonville & 08-07-2002 & 3 feet below surface & & & & & & & & & 5 \\
\hline & & & Mid-level/thermocline & & & & 0.79 & 0.03 & & $<.010$ & 0.02 & \\
\hline & & & Whole water column & & & & & & & & & \\
\hline \multirow[t]{4}{*}{424313083312005} & Big Lake at Andersonville & 04-15-2002 & 3 feet below surface & & & & 0.39 & 0.04 & & 0.170 & 0.01 & 4 \\
\hline & & & Mid-level/thermocline & 33 & & 5 & 0.42 & 0.03 & & 0.167 & 0.01 & 5 \\
\hline & & & $3 \mathrm{ft}$ off bottom & & & & 0.43 & 0.04 & & 0.166 & 0.01 & $<1.00$ \\
\hline & & & Whole water column & & & & & & & & & $<1.00$ \\
\hline \multirow[t]{4}{*}{424313083312005} & Big Lake at Andersonville & 08-07-2002 & 3 feet below surface & & & & 0.87 & 0.03 & & $<.010$ & 0.03 & 2 \\
\hline & & & Whole water column & & & & & & & & & 2 \\
\hline & & & Mid-level/thermocline & & & & 0.91 & 0.04 & & $<.010$ & 0.04 & 3 \\
\hline & & & $3 \mathrm{ft}$ off bottom & & & & 0.84 & 0.04 & & $<.010$ & 0.03 & 2 \\
\hline
\end{tabular}


Table 12. Results of chemical water-quality analyses for selected parameters at selected lakes in Oakland Ccounty, Michigan, 2002 - 2003, continued $\left[\mu \mathrm{s} / \mathrm{cm}\right.$, microsiemens per centimeter @ $25^{\circ} \mathrm{C} ; \mathrm{mg} / \mathrm{L}$, milligrams per liter; $\mu \mathrm{g} / \mathrm{L}$, micrograms per liter]

\begin{tabular}{|c|c|c|c|c|c|c|c|c|c|c|c|c|c|}
\hline Site Number & Lake Name & Sample Date & Position in water column & $\begin{array}{c}\text { Time } \\
\text { sampled }\end{array}$ & $\begin{array}{c}\text { Sampling } \\
\text { depth in } \\
\text { feet }\end{array}$ & 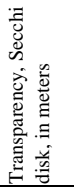 & 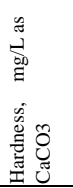 & 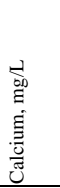 & 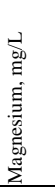 & 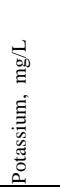 & 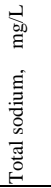 & 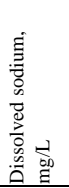 & 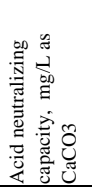 \\
\hline \multirow[t]{4}{*}{424335083405205} & Tipsico Lake & 09-08-2003 & 3 feet below surface & 1015 & 3 & 3.05 & & & & & & & \\
\hline & near Rose Center & & Mid-level/thermocline & 1020 & 12 & & & 22 & 7.46 & 1.2 & & 8.2 & \\
\hline & & & $3 \mathrm{ft}$ off bottom & 1025 & 17 & & & & & & & & \\
\hline & & & Whole water column & 1010 & 20 & & & & & & & & \\
\hline \multirow[t]{4}{*}{424634083143605} & Lake Orion near Lake Orion & 09-09-2003 & 3 feet below surface & 900 & 3 & 4.27 & & & & & & & \\
\hline & & & Mid-level/thermocline & 905 & 18 & & & 46.7 & 22.6 & 1.8 & & 32.1 & \\
\hline & & & Whole water column & 855 & 28 & & & & & & & & \\
\hline & & & $3 \mathrm{ft}$ off bottom & 910 & 50 & & & & & & & & \\
\hline \multirow[t]{4}{*}{424804083311305} & Valley Lake near Davisburg & $09-10-2003$ & 3 feet below surface & 845 & 3 & 4.57 & & & & & & & \\
\hline & & & Mid-level/thermocline & 850 & 17 & & & 37.8 & 23.7 & 1.5 & & 13.1 & \\
\hline & & & Whole water column & 840 & 30 & & & & & & & & \\
\hline & & & $3 \mathrm{ft}$ off bottom & 855 & 43 & & & & & & & & \\
\hline \multirow[t]{4}{*}{424828083400205} & Dickinson Lake near Holly & $09-10-2003$ & 3 feet below surface & 1205 & 3 & 3.05 & & & & & & & \\
\hline & & & Whole water column & 1200 & 20 & & & & & & & & \\
\hline & & & Mid-level/thermocline & 1210 & 20 & & & 47.9 & 23.4 & 1.6 & & 26.3 & \\
\hline & & & $3 \mathrm{ft}$ off bottom & 1215 & 61 & & & & & & & & \\
\hline \multirow[t]{4}{*}{424830083311805} & Heron Lake near Holly & 04-15-2003 & 3 feet below surface & 1030 & 3 & 3.4 & & & & & & & \\
\hline & & & Mid-level/thermocline & 1035 & 20 & & 200 & 42 & 24 & 1.5 & 17 & & 153 \\
\hline & & & Whole water column & 1025 & 22 & & & & & & & & \\
\hline & & & $3 \mathrm{ft}$ off bottom & 1040 & 39 & & & & & & & & \\
\hline \multirow[t]{4}{*}{424830083311805} & Heron Lake near Holly & $08-21-2003$ & 3 feet below surface & 1130 & 3 & 3.2 & & & & & & & \\
\hline & & & Mid-level/thermocline & 1135 & 18 & & & & & & & & \\
\hline & & & Whole water column & 1125 & 21 & & & & & & & & \\
\hline & & & $3 \mathrm{ft}$ off bottom & 1140 & 36 & & & & & & & & \\
\hline \multirow[t]{4}{*}{424952083084305} & Lakeville Lake near Lakeville & 09-09-2003 & 3 feet below surface & 1250 & 3 & 4.57 & & & & & & & \\
\hline & & & Mid-level/thermocline & 1255 & 17 & & & 26.3 & 23.6 & 0.9 & & 18 & \\
\hline & & & Whole water column & 1245 & 30 & & & & & & & & \\
\hline & & & $3 \mathrm{ft}$ off bottom & 1300 & 54 & & & & & & & & \\
\hline
\end{tabular}


Table 12. Results of chemical water-quality analyses for selected parameters at selected lakes in Oakland Ccounty, Michigan, 2002 - 2003, continued $\left[\mu \mathrm{s} / \mathrm{cm}\right.$, microsiemens per centimeter @ $25^{\circ} \mathrm{C} ; \mathrm{mg} / \mathrm{L}$, milligrams per liter; $\mu \mathrm{g} / \mathrm{L}$, micrograms per liter]

\begin{tabular}{|c|c|c|c|c|c|c|c|c|c|c|c|c|}
\hline Site Number & Lake Name & Sample Date & Position in water column & 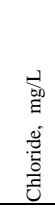 & 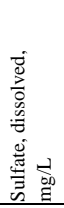 & 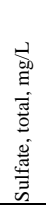 & 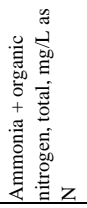 & 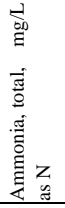 & 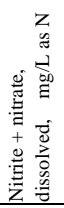 & 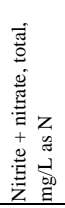 & 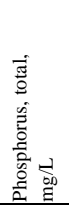 & 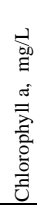 \\
\hline \multirow[t]{4}{*}{424335083405205} & Tipsico Lake & $09-08-2003$ & 3 feet below surface & & & & 0.72 & $<<.01$ & $<<.022$ & & $<.04$ & 11 \\
\hline & near Rose Center & & Mid-level/thermocline & 23.3 & 5.1 & & 0.72 & $<.01$ & $<.022$ & & $<.04$ & \\
\hline & & & $3 \mathrm{ft}$ off bottom & & & & 0.64 & $<.01$ & $<.022$ & & $<.04$ & 8 \\
\hline & & & Whole water column & & & & & & & & & 5 \\
\hline \multirow[t]{3}{*}{424634083143605} & Lake Orion near Lake Orion & 09-09-2003 & 3 feet below surface & & & & 0.57 & $<.01$ & $<.022$ & & $<.04$ & \\
\hline & & & Mid-level/thermocline & 66 & 32.5 & & 0.59 & $<.01$ & $<.022$ & & $<.04$ & 3.1 \\
\hline & & & $3 \mathrm{ft}$ off bottom & & & & 0.60 & 0.14 & 0.088 & & $<.04$ & \\
\hline \multirow[t]{3}{*}{424804083311305} & Valley Lake near Davisburg & 09-10-2003 & 3 feet below surface & & & & 0.77 & $<.01$ & $<.022$ & & $<.04$ & \\
\hline & & & $\begin{array}{l}\text { Mid-level/thermocline } \\
\text { Whole water column }\end{array}$ & 38.5 & 15 & & 0.67 & 0.02 & $<.022$ & & $<.04$ & \\
\hline & & & $3 \mathrm{ft}$ off bottom & & & & 2.10 & 1.14 & $<.022$ & & E.04 & 7.7 \\
\hline \multirow[t]{4}{*}{424828083400205} & Dickinson Lake near Holly & 09-10-2003 & 3 feet below surface & & & & 0.43 & 0.03 & 0.231 & & $<.04$ & \\
\hline & & & Whole water column & & & & & & & & & \\
\hline & & & Mid-level/thermocline & 49.8 & 31.2 & & 0.55 & 0.06 & 0.238 & & $<.04$ & \\
\hline & & & $3 \mathrm{ft}$ off bottom & & & & 1.40 & 0.77 & $<.022$ & & $<.04$ & \\
\hline \multirow[t]{4}{*}{424830083311805} & Heron Lake near Holly & $04-15-2003$ & 3 feet below surface & & & & 1.20 & 0.07 & & 0.081 & 0.01 & \\
\hline & & & Mid-level/thermocline & 46 & & 8 & 1.80 & 0.07 & & 0.083 & 0.01 & \\
\hline & & & Whole water column & & & & & OPO & & 0.456 & 001 & \\
\hline & & & J th on voturi & & & & 0.49 & 0.02 & & 0.400 & 0.01 & \\
\hline \multirow[t]{4}{*}{424830083311805} & Heron Lake near Holly & $08-21-2003$ & 3 feet below surface & & & & 1.30 & 0.04 & & $<.010$ & 0.01 & \\
\hline & & & Mid-level/thermocline & & & & 1.40 & 0.05 & & $<.010$ & 0.02 & \\
\hline & & & Whole water column & & & & & & & & & \\
\hline & & & $3 \mathrm{ft}$ off bottom & & & & 1.90 & E.43 & & $<.010$ & 0.04 & \\
\hline \multirow[t]{3}{*}{424952083084305} & Lakeville Lake near Lakeville & 09-09-2003 & 3 feet below surface & & & & 0.45 & $<.01$ & $<.022$ & & $<.04$ & \\
\hline & & & $\begin{array}{l}\text { Mid-level/thermocline } \\
\text { Whole water column }\end{array}$ & 39.9 & 25.6 & & 0.51 & $<.01$ & $<.022$ & & $<.04$ & \\
\hline & & & $3 \mathrm{ft}$ off bottom & & & & 1.20 & 0.60 & $<.022$ & & E.03 & \\
\hline
\end{tabular}


Table 13. Results of ecological assessment at selected river-channel locations in Oakland County, Michigan, September 2003

\begin{tabular}{|c|c|c|c|c|c|c|c|c|c|c|c|}
\hline $\begin{array}{l}\text { Station } \\
\text { number }\end{array}$ & Habitat & $\begin{array}{l}\text { Individual } \\
\text { organisms }\end{array}$ & Taxa & 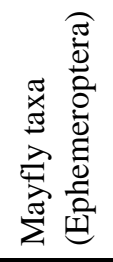 & 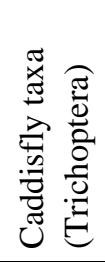 & 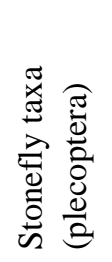 & 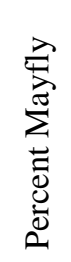 & 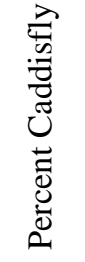 & 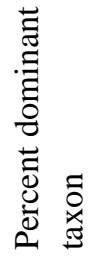 & 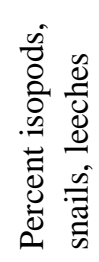 & 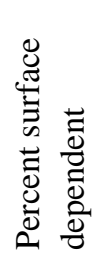 \\
\hline 04143830 & 139 & 156 & 20 & 1 & 3 & 1 & $2 \%$ & $9 \%$ & $22 \%$ & $8 \%$ & $24 \%$ \\
\hline 04148035 & 136 & 70 & 19 & 2 & 1 & 0 & $9 \%$ & $4 \%$ & $14 \%$ & $13 \%$ & $56 \%$ \\
\hline 04160800 & 137 & 122 & 14 & 1 & 2 & 0 & $1 \%$ & $25 \%$ & $39 \%$ & $16 \%$ & $10 \%$ \\
\hline 04160900 & 108 & 110 & 13 & 0 & 2 & 0 & $0 \%$ & $10 \%$ & $26 \%$ & $20 \%$ & $19 \%$ \\
\hline 04161000 & 95 & 123 & 16 & 1 & 1 & 0 & $8 \%$ & $34 \%$ & $34 \%$ & $6 \%$ & $4 \%$ \\
\hline 04161540 & 97 & 46 & 11 & 2 & 3 & 0 & $9 \%$ & $57 \%$ & $52 \%$ & $0 \%$ & $4 \%$ \\
\hline 04161580 & 163 & 105 & 20 & 0 & 1 & 1 & $0 \%$ & $3 \%$ & $29 \%$ & $7 \%$ & $11 \%$ \\
\hline 04161810 & 82 & 86 & 11 & 0 & 1 & 0 & $0 \%$ & $19 \%$ & $52 \%$ & $8 \%$ & $1 \%$ \\
\hline 04166000 & 111 & 208 & 17 & 1 & 2 & 0 & $1 \%$ & $31 \%$ & $17 \%$ & $15 \%$ & $19 \%$ \\
\hline 04166100 & 109 & 45 & 11 & 0 & 1 & 0 & $0 \%$ & $2 \%$ & $33 \%$ & $29 \%$ & $11 \%$ \\
\hline 04166200 & 89 & 54 & 10 & 0 & 1 & 0 & $0 \%$ & $2 \%$ & $56 \%$ & $13 \%$ & $6 \%$ \\
\hline 04166315 & 137 & 62 & 18 & 2 & 1 & 0 & $4 \%$ & $8 \%$ & $21 \%$ & $6 \%$ & $47 \%$ \\
\hline 04170000 & 136 & 104 & 21 & 3 & 1 & 0 & $4 \%$ & $1 \%$ & $32 \%$ & $15 \%$ & $7 \%$ \\
\hline 04170500 & 148 & 138 & 11 & 0 & 1 & 0 & $0 \%$ & $7 \%$ & $28 \%$ & $33 \%$ & $11 \%$ \\
\hline
\end{tabular}


